\title{
Investigational Chemotherapy and Novel Pharmacokinetics for the Treatment of Cancers of the CNS
}

Neal Shah

Follow this and additional works at: https://researchrepository.wvu.edu/etd

\section{Recommended Citation}

Shah, Neal, "Investigational Chemotherapy and Novel Pharmacokinetics for the Treatment of Cancers of the CNS" (2018). Graduate Theses, Dissertations, and Problem Reports. 7250.

https://researchrepository.wvu.edu/etd/7250

This Dissertation is protected by copyright and/or related rights. It has been brought to you by the The Research Repository @ WVU with permission from the rights-holder(s). You are free to use this Dissertation in any way that is permitted by the copyright and related rights legislation that applies to your use. For other uses you must obtain permission from the rights-holder(s) directly, unless additional rights are indicated by a Creative Commons license in the record and/ or on the work itself. This Dissertation has been accepted for inclusion in WVU Graduate Theses, Dissertations, and Problem Reports collection by an authorized administrator of The Research Repository @ WVU.

For more information, please contact researchrepository@mail.wvu.edu. 


\title{
Investigational Chemotherapy and Novel Pharmacokinetics for the Treatment of Cancers of the CNS
}

\author{
Neal Shah, Pharm.D. \\ Dissertation submitted \\ to the School of Pharmacy \\ at West Virginia University \\ in partial fulfillment of the requirements for the degree of \\ Doctor of Philosophy in \\ Pharmaceutical and Pharmacological Sciences
}

\author{
Paul R. Lockman, Ph.D., Chair \\ Erik A. Bey, Ph.D., \\ Christopher P. Cifarelli, M.D., Ph.D., \\ Lori A. Hazlehurst, Ph.D., \\ William Petros, Pharm.D. \\ Department of Pharmaceutical and Pharmacological Sciences \\ Robert C. Byrd Health Sciences Center
}

\author{
Morgantown, West Virginia \\ 2018
}

Keywords: Brain metastases, breast cancer, lung cancer, blood-tumor barrier, chemotherapy

Copyright 2018 Neal Shah 


\section{ABSTRACT \\ Investigational Chemotherapy and Novel Pharmacokinetics for the Treatment of Cancers of the CNS Neal Shah, Pharm.D.}

This dissertation (a) detailed the optimal imaging times and quantifiable intervals of the non-toxic tumor surrogate marker D-luciferin in metastatic and primary brain tumors, (b) characterized the creation, passive permeability, efflux pump status, and vascularity of a widely-used EGFR-mutant lung cancer cell line, (c) evaluated the in-vitro cytotoxicity and in-vivo efficacy of three novel chemotherapeutic agents on brain metastases of lung and breast cancer as well as primary glioblastoma, and (d) provided a review of the current clinical and preclinical treatment options for breast cancer and lung cancer brain metastases, and primary glioblastoma. 


\section{ACKNOWLEDGEMENTS}

The work presented in this dissertation would have been unachievable without collective and collaborative effort. I am eternally grateful for all the help I have received through the years.

First, I would like to profoundly thank my mentor Dr. Paul Lockman. Without your guidance through these three years I would be completely lost. Your support has meant the world to me, from my initial uneasiness in the first year to surprising you in your office and saying I wanted to publish papers and graduate in five months. I have learned so much from you, not only in the field of science, but life in general. You remain creative in scientific approach and data publication, which is something I am slowly starting to incorporate into my life. Your flexibility of balancing research and life has allowed me to explore life and pursue the field of medicine I wish to practice. I feel a connectedness as our backgrounds are both in clinical practice and this allows us to balance the mindset of clinician scientists. I enjoyed all of our time together and I hope to collaborate with you in the future. Thank you, Dr. Julie Lockman, for working with me through the hectic race at the end for my Masters. You both have shown me the virtues of patience, hard work, determination, and how an ideal partnership works.

I would also like to thank all the members in my committee for their assistance throughout the time spent in my PhD. Dr. Cifarelli, Dr. Hazlehurst, Dr. Petros and Dr. Bey, you reviewed my grant application, provided letters of support and recommendation, gave me recommendations for experimental approach and creative suggestions to make my work more impactful. You have seen my progress throughout graduate school and I hope I have adequately evolved as a scientist in your eyes. 
To my seniors, the postdoctoral scholars: Dr. Chris Adkins, where to begin? We bonded over Indian Pale Ales and Game of Thrones, hot sauce, exercise, and transitioned from being labmates to friends. One of the most important things I learned from you is how to be critical of scientific work, especially your own. Thank you for all of your patience during the transition of my first year, and congratulations again on your position as a pharmacy school professor. If I move away from Morgantown, I will be sure to eat some Peppebronis in your honor. Dr. Raj Jagannathan, you had a simple motto: "make science easy". Thank you both so much.

To my fellow Lockman Lab PhD graduates: It has been a journey, hasn't it? Dr. Tori Terrell-Hall, though our time was limited, it was fantastic. I enjoyed seeing you problem solve and balance your children and lab work. If I am ever in Texas, I will definitely contact you! Besides, I think I owe you quite a bit of money from the swear jar. Dr. Afroz Mohammad, there are no shortage of fond memories with you. Besides Dr. Lockman, I have spent the most time in lab with you, and you have changed many life views I held. You stayed very late, long beyond your own long days to help me out with perform essential procedures creating figures. Even after you defended your dissertation, up until the day you left, you helped me out. Your upbeat and constantly positive attitude has shown me there is utility in being optimistic. We laugh more than I thought was possible. There are so many, most likely too many amazing conversations and quotes: your "vegan" diet, going to a bar and ordering shots of water, coats-quotes, me being "hot" from radiation.... you remain an essential part of the Lockman Lab, and we will stay in contact!

To the future doctors: Rachel Tallman, I owe much of this dissertation to you. Without your countless hours of slicing brains and initial imaging, much of this work would have taken another full year. We have had some great times together, from 
working out and running to bar-hopping and that one time, hammocking. I am so proud of you for getting into medical school and I promise I will continue to yell at you when I see you in the hospital. Memorable quote: “There's no 'a' in orange! Except in the middle..." Dr. Katie Jarrell, I genuinely think you have made me better, nicer, and more human. It is always great to hang out with you, discuss life or movies or Game of Thrones, your future and pharmacy career plans, and so much more. You created "Neal on Wheels"! I am looking forward to all of our graduations and seeing where we all go throughout life. You both have helped me out so much!

To the current Lockman Lab PhD students: Samuel Sprowls, thank you for making me push myself harder in all aspects of life, especially exercise. I will never forget the day you helped me move and figure out how apartment addresses work. You and Chris may be the only people who like Game of Thrones more than I do. Your most memorable quote for when I hit a new lifting record: "Me: Guess what I got on inner leg? Sam: Herpes?" Mark Pinti, thank you for staying all those long hours in the IVIS and helping me out with imaging and weighing. Pushkar Saralkar, thank you for all your assistance with $\mathrm{IC}_{50}$ and experimental analysis, as well as showing me how to play tennis. I look forward to seeing how massive the Plastic Throne will be. Schuyler Vickers, it will be great to see where your projects go, and what types of meat you "have your way with". Without the assistance provided by you four, this dissertation would be incomplete.

To the former undergraduates, who are now pursuing a PhD: Emma Dolan, I am not surprised you ended up with acceptances at Scripps and Duke! It was great catching up with you in DC for AACR. Jessie Griffith, your most memorable quote is undoubtedly "How many doctorates does it take to move a cart?" The world may never know this answer, though I have confirmed it is not three. I hope you are staying warm in 
Minnesota. Thank you both for all the memories and for help in the initial years of my $\mathrm{PhD}$.

To the imaging facility, employees of OLAR, and members of the Pugacheva, Gibson, and Hazlehurst labs: Thank you for all the patience and assistance with these massive projects. It was frustrating and time-consuming to juggle the hundreds of animals we did in such a short time span, but you helped me get data I needed.

To Dr. Szklarz, Dr. Salati, Dr. Siderovski, and Dr. Geldenhuys: Thank you for always being in my corner and being flexible. Even when my ambition surpassed a passable possibility, you believed in me and helped me make this fervor into reality. I hope I have made you proud! To Dr. Kolodney, thank you for showing me how a real $\mathrm{MD} / \mathrm{PhD}$ scientist is supposed to be. I focus more on clinical aspects, but you reminded me that a clinician scientist always keeps basic science in the back of their mind. It is a lifelong balancing act, and I hope to emulate your career.

To the current MD/PhD students: Dr. Brandon Lucke-Wold, every student needs a peer mentor not only for guidance and as a role model, but to form a bond with, and to share life experiences with. You have pushed your own career further than is humanly possible and urged me to do the same. Thank you for taking time to talk with me, address my concerns, provide me with focus, and ultimately push me along the path of apotheosis. To Jessica Clemente, thank you for staying in touch and commiserating with me all this time. I will be sure to mimic Brandon and pass down helpful information as I finish the latter half of medical school. To Jesse Sundar, it is always great catching up at the gym. I look forward to seeing where you three end up. To Dr. Joshua Farris, Dr. Philip Pifer, Dr. Daniel Vanderbilt, Dr. Sriganesh Sharma, Dr. Mark Farrugia, Dr. Sylwia Brooks, thank you all for helping me out with lab mentors-you really hit a home run there! I look forward to seeing where your careers take you. 
How can it be possible that three years have passed since I took Step 1 and began my $\mathrm{PhD}$ ? I was rigid in thought and attitude, while demanding perfection and embodying frustration. Admittedly not much has changed in these areas, but I have become much more flexible, understanding, and open-minded. Though I am not the most intelligent scientist, I am now confident that I am one of the most motivated, focused, and ambitious, which will continue to serve me well throughout life.

In graduate school, there are weeks—often months—-where experiments don't work, data collected is unusable, and there doesn't seem to be a light at the end of the tunnel. The highs can be high; the lows can be low. Then, you factor in the rest of life and the scenario seems insurmountable. But though the hardest of these three doctorates, I have figured out how life really works: it is how we respond and react to hardships that defines who we are. Life is never going to be completely smooth. We prove we can handle the hurdles of life and emerge with some semblance of sanity.

The time spent and experiences I have shared with all of you have changed who I am as a person for the better. The years spent in my PhD have been the best years of my life, and I would not have done anything differently.

[29] years of pain

And I can feel it closing in

The will to rise above

Tearing my insides out

At the Gates

Cold

Slaughter of the Soul 


\section{TABLE OF CONTENTS}

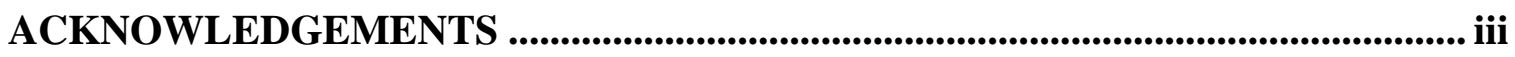

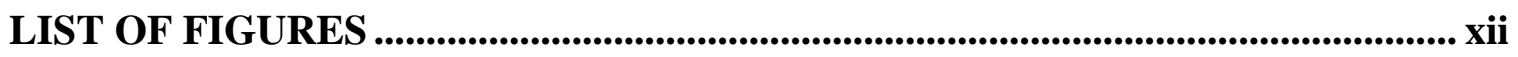

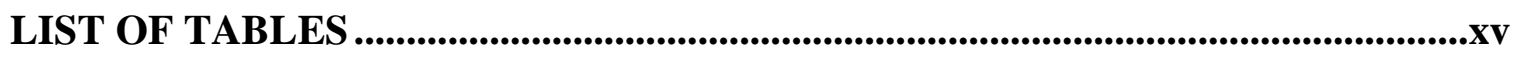

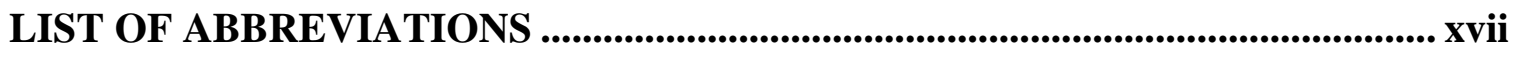

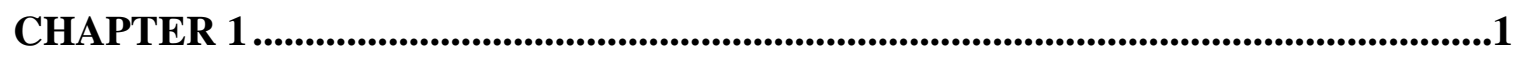

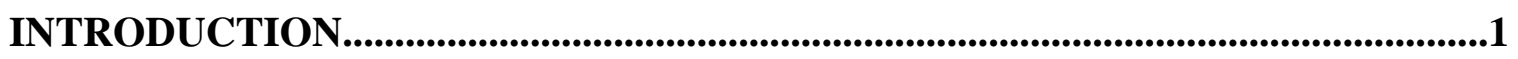

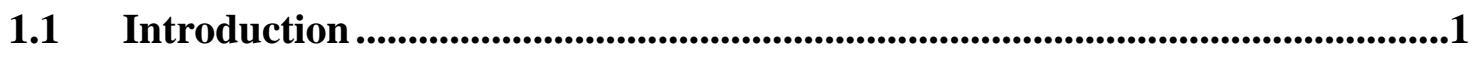

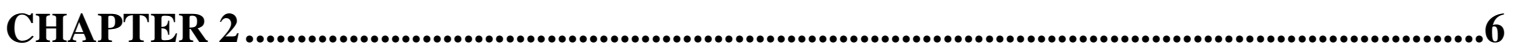

CURRENT TREATMENT STRATEGIES FOR LUNG CANCER AND BREAST CANCER BRAIN METASTASES AND GLIOBLASTOMA.....................................6

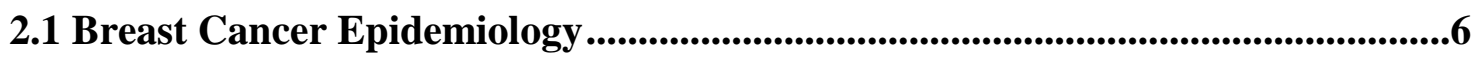

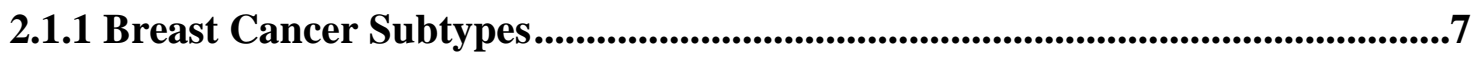

2.1.2 Breast Cancer Brain Metastases ...............................................................9

2.1.3 Conventional Breast Cancer Brain Metastases Chemotherapy ......................10

2.1.4 Targeted Breast Cancer Brain Metastases Chemotherapy ................................13

2.1.5 Immunotherapy for Breast Cancer Brain Metastases...................................15

2.2 Lung Cancer Epidemiology ....................................................................................18

2.2.1 Lung Cancer Subtypes......................................................................................19

2.2.2 Lung Cancer Brain Metastasis ...............................................................23

2.2.3 Conventional Lung Cancer Brain Metastases Chemotherapy .......................25

2.2.4 Targeted Lung Cancer Brain Metastases Chemotherapy ..............................26

2.2.5 Immunotherapy for Lung Cancer Brain Metastases..........................................32

2.3 Glioblastoma Epidemiology 
2.3.2 Immunotherapy for Glioblastoma .....................................................................36

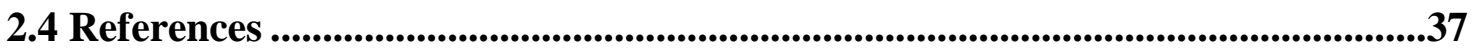

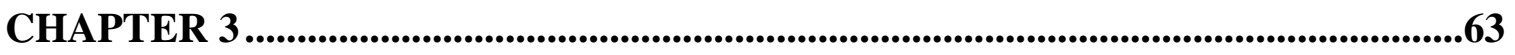

PHARMACOKINETICS OF LUCIFERIN IN PRECLINICAL BRAIN METASTASES AND PRIMARY BRAIN TUMOR MODELS ...............................63

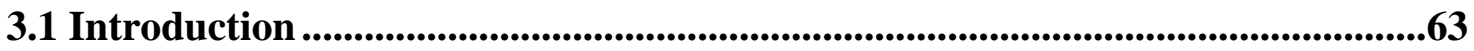

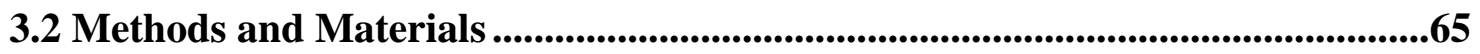

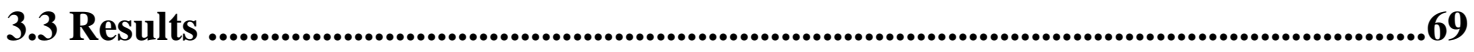

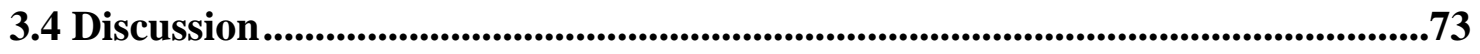

3.5 Conclusion ...........................................................................................................................79

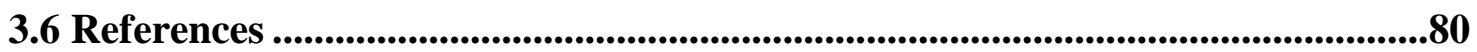

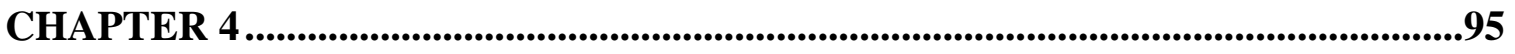

EFFECTS OF ETIRINOTECAN PEGOL (NKTR-102) IN THREE PRECLINICAL BREAST CANCER BRAIN METASTASES MODELS .....................................95

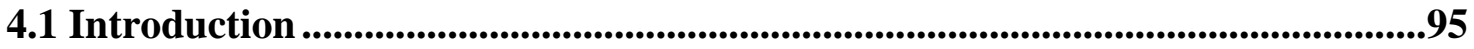

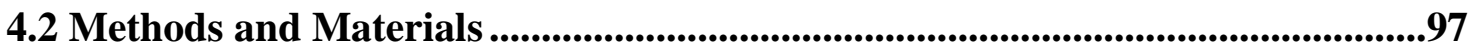

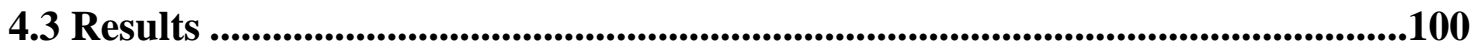

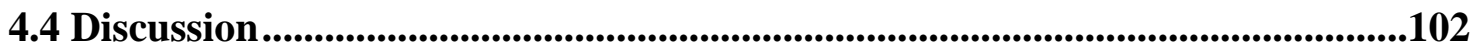

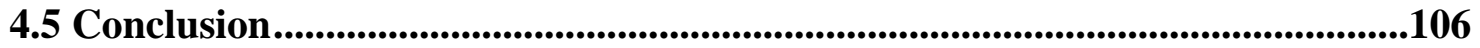

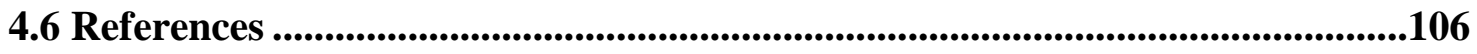

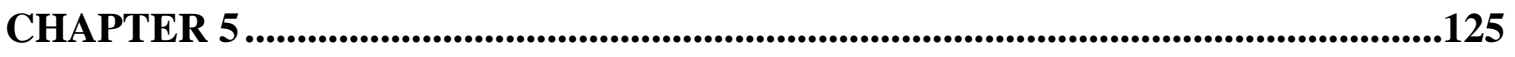

CHARACTERIZATION OF PRECLINICAL PC-9 LUNG CANCER BRAIN

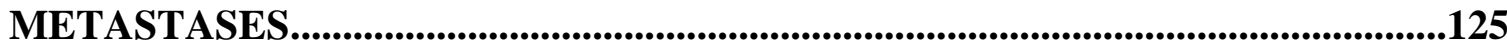


5.1 Introduction

5.2 Methods and Materials

5.3 Results

5.4 Discussion

5.5 Conclusion 141

5.6 References

CHAPTER 6 .166

EFFECTS OF ETIRINOTECAN PEGOL (NKTR-102) IN THE PC-9

PRECLINICAL LUNG CANCER METASTASES MODEL 166

6.1 Introduction .166

6.2 Methods and Materials 167

6.3 Results

6.4 Discussion.

6.5 Conclusion 174

6.6 References 175

CHAPTER 7

PRELIMINARY DATA OF ETIRINOTECAN PEGOL (NKTR-102) EFFICACY

IN A PRECLINICAL GLIOBLASTOMA MODEL .192

7.1 Introduction .192

7.2 Methods and Materials . .193

7.3 Results . .194

7.4 Discussion .195

7.5 Conclusion 197 
7.6 References ...............................................................................................................198

CHAPTER 8

PRELIMINARY DATA OF ABRAXANE AND DOXIL EFFICACY IN

PRECLINICAL BRAIN METASTASES MODELS ............................................203

8.1 Introduction ...........................................................................................................203

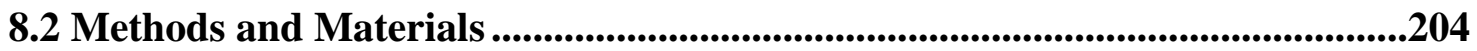

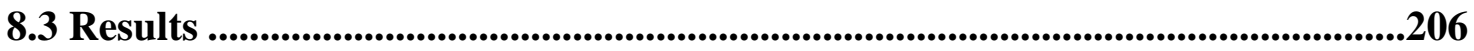

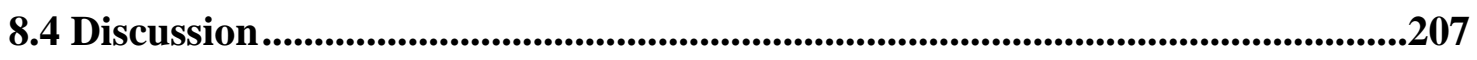

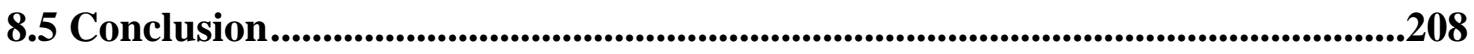

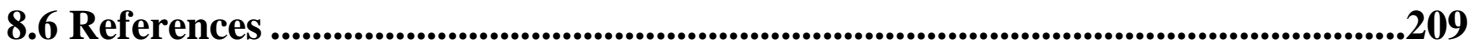

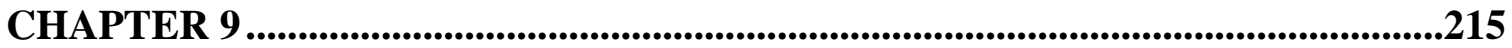

CONCLUSION AND FUTURE DIRECTIONS .............................................215

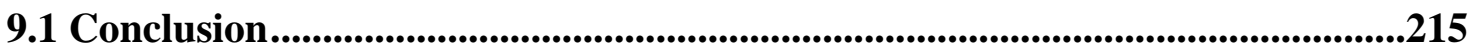

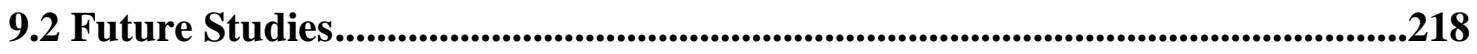




\section{LIST OF FIGURES}

Figure 3.1. Representative time-course of bioluminescence capture and quantification. .86

Figure 3.2. Radiance luciferin pharmacokinetics for brain tumor models. .87

Figure 3.3. Normalized luciferin bioluminescence profiles for brain tumor models. 88

Figure 3.4. In vitro luciferin kinetics corresponds with in vivo bioluminescence......89

Figure 3.5. Radiance and normalized AUC comparisons. .90

Figure 4.1. Etirinotecan pegol reduces tumor burden in the MDA-MB-231-Br model compared to conventional chemotherapy and vehicle.

Figure 4.2. Kaplan-Meier survival curve of MDA-MB-231-Br mice treated with etirinotecan pegol and conventional chemotherapy.

Figure 4.3. Etirinotecan pegol reduces tumor burden in the JIMT-1-Br model compared to lapatinib and vehicle.

Figure 4.4. Kaplan-Meier survival curve of JIMT-1-Br mice treated with etirinotecan pegol and conventional chemotherapy.

Figure 4.5. Etirinotecan pegol does not significantly reduce tumor burden in the 4T1-Br model compared to vehicle.

Figure 4.6. Kaplan-Meier survival curve of 4T1-Br mice treated with etirinotecan pegol and conventional chemotherapy

Figure 4.7. Accumulation of irinotecan in tumors and normal brain from ${ }^{14} \mathrm{C}$ etirinotecan pegol. 
Figure 5.1: Distribution and survival of the brain-seeking PC-9 cell line. 146

Figure 5.2. Oregon Green is a P-glycoprotein substrate.

Figure 5.3. Distribution of brain metastases in PC-9-Br...................................... 148

Figure 5.4. Ventricular infiltration leads to spinal tumor burden. ......................... 149

Figure 5.5. PC-9-Br is less sensitive to chemotherapy compared to the parenteral line. 150

Figure 5.6. Permeability changes of PC-9-Br treated with vehicle. 151

Figure 5.7. Passive permeability changes of PC-9-Br treated with cisplatinetoposide. 152

Figure 5.8. Passive permeability changes of PC-9-Br treated with cisplatinpemetrexed. 153

Figure 5.9. Passive permeability changes of PC-9-Br treated with gefitinib. 154

Figure 5.10. Passive permeability changes of PC-9-Br treated with afatinib 155

Figure 5.11. Passive permeability changes of PC-9-Br treated with osimertinib... 156 Figure 5.12. Comparison of passive permeability between PC-9-Br treatment groups. 157

Figure 5.13. Distribution of PC-9-Br tumors. 158

Figure 6.1. Sensitivity to SN-38 for three brain-seeking lung cancer lines. . .178

Figure 6.2. Bioluminescence in PC-9-Br treated with BEACON therapy. . .180 Figure 6.3. Kaplan-Meier survival curve of PC-9-Br mice treated with etirinotecan pegol and BEACON-based chemotherapy. .181 
Figure 6.4. Bioluminescence in conventional lung chemotherapy in the PC-9-Br model.

Figure 6.5. Kaplan-Meier survival curve of PC-9-Br mice treated with conventional lung chemotherapy .184

Figure 6.6. Bioluminescence profiles of EGFR-inhibitors in the PC-9-Br model....186 Figure 6.7. Kaplan-Meier survival curve of PC-9-Br mice treated with targeted lung chemotherapy .187

Figure 7.1. $\mathrm{U} 251$ is sensitive to $\mathrm{SN}-38$ in the nanomolar range. .200 Figure 7.2. NKTR-102 enhances survival compared to vehicle therapy in the U251 model. .201 Figure 7.3. Etirinotecan pegol reduces tumor burden and improves survival compared to vehicle in the $\mathrm{U} 251$ glioblastoma model. .202

Figure 8.1. In-vitro cytotoxicity of Abraxane and Doxil on MDA-MB-231-Br cells. .211

Figure 8.2. MDA-MB-231-Br survival is not improved on Abraxane therapy . .212 Figure 8.3. Doxil bioluminescence and survival effects on MDA-MB-231-Br tumors. .213

Figure 8.4. Effects of Doxil on the PC-9-Br model. .214 


\section{LIST OF TABLES}

Table 3.1. Timing of luciferin imaging for in vivo cancer cell models. ......................91

Table 3.2. Radiance pharmacokinetic data for tumor models. ......................................92

Table 3.3. Normalized pharmacokinetic data for tumor models...................................93

Table 3.4. Signal range during bioluminescence imaging. ................................................94

Table 4.1. Chemotherapy formulations and dosage schedules for NKTR-102

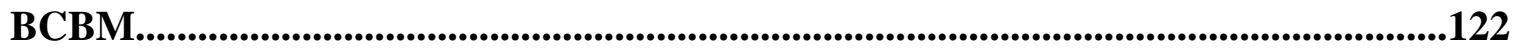

Table 4.2. In-vitro cytotoxicity assay of chemotherapy in cancer cell lines. ..............123

Table 4.3. Survival analysis of chemotherapy and vehicle.....................................124

Table 5.1. In-vitro chemotherapeutic sensitivity comparisons of the parenteral and

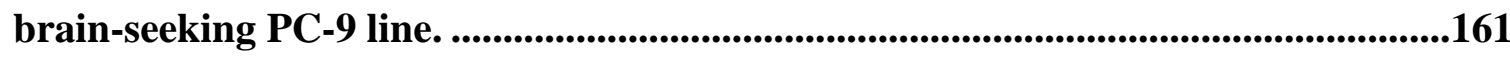

Table 5.2. Sizes of PC-9-Br tumors based on drug treatment. ....................................162

Table 5.3. ${ }^{14} \mathrm{C}$-AIB fold permeability is less in groups that improve median

survival.

Table 5.4. Oregon Green fluorescence intensity does not correlate with median

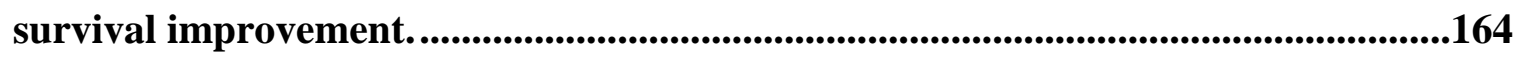

Table 5.5. ICG fluorescence intensity is similar in all treatment groups..................165

Table 6.1. Formulations and dosing schedule for NKTR-102 in the PC-9-Br model. 
Table 6.2. Survival data of NKTR-102 against chemotherapy in the PC-9-Br model. ....... 


\section{LIST OF ABBREVIATIONS}

\begin{tabular}{|c|c|}
\hline AUC & Area under the curve \\
\hline ALK & Anaplastic lymphoma kinase \\
\hline $\mathrm{BBB}$ & Blood-brain barrier \\
\hline $\mathrm{BCBM}$ & Breast cancer brain metastases \\
\hline BTB & Blood-tumor barrier \\
\hline CSF & Cerebrospinal fluid \\
\hline CTLA-4 & Cytotoxic T-lymphocyte associated protein- 4 \\
\hline EGFR & Epidermal growth factor receptor \\
\hline EML-4 & Echinoderm microtubule-associated protein- 4 \\
\hline EPR & Enhanced permeation and retention \\
\hline ER & Estrogen receptor \\
\hline Fluc & Firefly luciferase \\
\hline HER2 & Human epidermal growth factor receptor 2 \\
\hline LCBM & Lung cancer brain metastases \\
\hline NSCLC & Non-small cell lung cancer \\
\hline PD & Programmed death receptor \\
\hline PD-L1, -2 & Programmed death receptor ligand-1, -2 \\
\hline P-gp & Permeability glycoprotein \\
\hline PR & Progesterone receptor \\
\hline SCLC & Small cell lung cancer \\
\hline T-DM1 & Trastuzumab emtansine \\
\hline TNBC & Triple negative breast cancer \\
\hline WBRT & Whole brain radiation therapy \\
\hline
\end{tabular}




\section{CHAPTER 1}

\section{INTRODUCTION}

\subsection{Introduction}

It is odd to think of cancer as a unifier, but in some ways, it is. Cancer has remained the second-most common cause of death since the 1980s, disregarding sex, ethnicity, race, or characteristics otherwise. [1] In 2016, cancer was responsible for nearly 600,000 deaths, and with life expectancy increasing, the incidence of cancer diagnosis and deaths have also increased. [1] In women breast cancer is most common, while in men prostate cancer is most common; in both genders this is followed by lung cancer and colorectal cancer. [2] For cancer deaths, in both men and women lung cancer is first, followed by prostate cancer or breast cancer, respectively, and then colorectal cancer. [2]

Cancer within its tissue of origin is defined as "primary", spreading to other organs in a process known as metastasis. [3] It is metastasis that is responsible for the lethality of cancer and the magnitude of cancer death. [4] While cancer may metastasize to any location, the highest incidence of metastases occurs in the lung, liver, bone, brain, and adrenal glands. [5] Depending on cancer type, approximately 10 to $50 \%$ of all cancer patients will eventually develop a metastasis. [6-8]

Treatments for metastases are dependent on tumor location but generally involve a regimen of radiation, surgery, and chemotherapy. Brain metastases becomes increasingly challenging to treat due to the physiochemical blood-brain barrier chemotherapy is limited in its cytotoxic ability, and in combination with the invasiveness of resection. [9] 
Once diagnosed with brain metastases, the median survival typically ranges from three months to two years. [10]

Fortunately, novel chemotherapeutic agents, redesigned dosage forms, and new methods of drug administration have allowed for modest improvement of survival in brain metastases. These new agents often act as antagonists of a pathway upregulated in cancer cells (such as osimertinib, which inhibits the EGFR signaling pathway), prolong and enhance cytotoxic effects by conjugation to linker molecules (such as etirinotecan pegol, which is a tetramer of irinotecan linked by polyethylene glycol chains), or avoid efflux pumps to penetrate the brain and tumor (such as Doxil, which is a pegylated liposomal version of doxorubicin). Trends have shown that more molecules are being developed with the intent to cross the blood-brain and blood-tumor barriers to treat brain metastases.

In this dissertation, we investigate the effect of novel chemotherapeutic agents compared to standard-of-care chemotherapy on median survival of two models of brain metastases and one model of glioblastoma. We additionally correlate in vitro toxicity assays to in vivo drug efficacy and characterize the optimal timing for luciferin bioluminescence quantification and its surrogacy for tumor burden in response to chemotherapy. As our laboratory has extensively characterized the blood-tumor barrier of breast cancer brain metastases [6], initial characterization of the blood-tumor barrier of a EGFR-mutated lung cancer brain metastases model is described. Effects of chemotherapeutic agents on barrier vascular integrity and efflux pumps are visualized using fluorescence and autoradiography data. 
Chapter 2 reviews the epidemiology, subtypes, prognostic factors, and survival characteristics of brain metastases from breast cancer and lung cancer, as well as primary glioblastoma. Conventional chemotherapeutic regimens, novel chemotherapy, and in-trial agents are also discussed.

Chapter 3 details optimal pharmacokinetic timing for luciferin imaging for breastbrain, lung-brain, and glioblastoma models used subsequently for the rest of these dissertation experiments.

Chapter 4 describes the survival effects of pegylated irinotecan (etirinotecan pegol) in three models of breast cancer brain metastases. Etirinotecan pegol is hypothesized to have preferential accumulation in tumors due to the vasculature leakiness, and may additionally bypass efflux transporters. In vitro toxicity effects as well as in vivo survival effects of etirinotecan pegol are compared to BEACON trial agents for the respective models. Bioluminescence curves are similarly compared for treatment groups.

Chapter 5 illustrates the creation of the PC-9-Br EGFR brain-seeking lung cancer cell line. Luciferin kinetics reveal the best range of time for in vivo imaging to assess tumor burden and response to treatments. Fluorescence of tomato red (tumor), Oregon Green (efflux pump), and indocyanine green (vascularity) characterize the environment of lung cancer brain metastases, and effects of chemotherapy on this environment. The effects of the permeability glycoprotein efflux pump at the blood-tumor barrier is visualized and inhibited to show its contribution to chemotherapy restriction. Autoradiography data shows restricted tumor accumulation of chemotherapeutic agents, which correlates with observed survival. 
Chapter 6 depicts the survival effects of etirinotecan pegol in the EGFR-mutated PC9-Br model of lung cancer brain metastases. In vitro toxicity assays of BEACON agents, as well as conventional chemotherapy used to treat lung cancer, show sensitivity and estimated response of the PC9 line to treatments. Results of survival and bioluminescence are compared to cellular sensitivity. The differences in permeability, in vitro toxicity, and in vivo survival are discussed, especially in the context of EGFRtargeted tyrosine kinase inhibitors.

Chapter 7 reports the survival effects of etirinotecan pegol in the setting of primary gliobastoma. In contrast to brain metastases models, the glioblastoma model uses intracranial injections and a slower-growing tumor, which may favor chemotherapy deposition.

Chapter 8 describes the in vitro and in vivo effects of nano-albumin-bound paclitaxel and pegylated liposomal docetaxel on the triple negative breast cancer and lung cancer models. Chapter 7 and Chapter 8 feature preliminary data, with in vivo comparisons to conventional chemotherapy ongoing.

In summary, this dissertation evaluates the current treatment strategies and effects of three novel chemotherapeutic agents featuring enhanced brain permeability on the survival of preclinical brain metastases and primary lesions. This dissertation also characterizes the vascular integrity, passive permeability, efflux pump contribution, and overall environment of brain metastases of EGFR-mutant lung cancer, which provides insight into why patients on chemotherapy often have relapsing lesions. When viewed altogether, the data emphasizes a multifocal approach to improve penetrance of 
chemotherapy by molecular design or innovative formulation, with the goal of minimizing damage to normal brain parenchyma surrounding tumors.

1. National Center for Health Statistics, U.S., in Health, United States, 2016: With Chartbook on Long-term Trends in Health. 2017: Hyattsville (MD).

2. Siegel, R.L., K.D. Miller, and A. Jemal, Cancer Statistics, 2017. CA Cancer J Clin, 2017. 67(1): p. 7-30.

3. Hanahan, D. and R.A. Weinberg, Hallmarks of cancer: the next generation. Cell, 2011. 144(5): p. 646-74.

4. Seyfried, T.N. and L.C. Huysentruyt, On the origin of cancer metastasis. Crit Rev Oncog, 2013. 18(1-2): p. 43-73.

5. Budczies, J., et al., The landscape of metastatic progression patterns across major human cancers. Oncotarget, 2015. 6(1): p. 570-83.

6. Weigelt, B., J.L. Peterse, and L.J. van 't Veer, Breast cancer metastasis: markers and models. Nat Rev Cancer, 2005. 5(8): p. 591-602.

7. Witzel, I., et al., Breast cancer brain metastases: biology and new clinical perspectives. Breast Cancer Res, 2016. 18(1): p. 8.

8. Dawe, D.E., J.N. Greenspoon, and P.M. Ellis, Brain metastases in non-small-cell lung cancer. Clin Lung Cancer, 2014. 15(4): p. 249-57.

9. Lockman, P.R., et al., Heterogeneous blood-tumor barrier permeability determines drug efficacy in experimental brain metastases of breast cancer. Clin Cancer Res, 2010. 16(23): p. 5664-78.

10. Stelzer, K.J., Epidemiology and prognosis of brain metastases. Surg Neurol Int, 2013. 4(Suppl 4): p. S192-202. 


\section{CHAPTER 2}

\section{CURRENT TREATMENT STRATEGIES FOR LUNG CANCER AND BREAST CANCER BRAIN METASTASES AND GLIOBLASTOMA}

\subsection{Breast Cancer Epidemiology}

Breast cancer remains the most common malignancy in women. Over an 80-year lifetime, the National Cancer Institute's Surveillance, Epidemiology, and End Results reports from 2003 to 2007 report the absolute risk of a woman developing breast cancer is 1 -in-8 (12\%), which is a modest increase from the lifetime risk in the 1970s of approximately 1-in-10 (10\%). [1] The strongest risk factor for developing breast cancer is age, with incidence increasing as age increases. [1] In their 30s, women have less than $1 \%$ risk of developing breast cancer, yet in their 70 s the risk increases to nearly $4 \%$. [1] Other risk factors that increase the incidence of breast cancer include family history of breast cancer, early menstruation, late menopause, use of hormonal therapy, obesity, Caucasian race, physical inactivity, excessive alcohol intake, smoking, radiation therapy of the chest, and inherited genetic mutations. [1]

In 2017, it is estimated that more than 250,000 new cases of breast cancer will be diagnosed and more than 40,000 women will die from breast cancer. [2] From 1989 to 2015, the overall death rates of breast cancer decreased by 39\% and from 2006 to 2015 in all racial and ethnic groups. [2] The rapid decline in mortality is attributed to improved screening via early mammography and the widespread use of chemotherapy. [2] The seminal discovery of human epidermal growth factor receptor 2 (HER2) presence in up to 
$30 \%$ of breast tumors (as well as high levels in other tumor locations) in the mid-1980s lead to the production of Herceptin (trastuzumab), the first antibody against HER2. [3] This discovery significantly contributed to the rapid mortality reduction from breast cancer, as well as introduced a new risk factor for breast cancer metastasis. A conundrum formed: while non-specific chemotherapy and targeted therapy such as trastuzumab may provide primary control of breast cancer, these agents do not readily achieve adequate cytotoxic concentrations in the brain, and allow seeding into the brain and brain metastases development. [4] With the discovery of HER2, the molecular subtype of breast cancer was found to influence formation of brain metastases as well as overall prognosis.

\subsubsection{Breast Cancer Subtypes}

Breast tissue is responsive to hormones such as estrogen and progesterone as part of normal physiologic mechanisms. While estrogen stimulates growth of the ductal tissue components, lobular development is driven by progesterone. [5] Dysregulations of the estrogen receptor (ER), progesterone receptor (PR), and their signaling pathways have been attributed to development of breast cancer and are termed "hormone receptor positive". [6] With the incorporation of HER2 as both a risk factor and prognostic factor, the hormone receptor breast cancer split into two further types: Luminal A and Luminal B.

Luminal A represent tumors that are ER and PR positive, but do not possess HER2 upregulation (are HER2 negative), while Luminal B tumors retain ER, PR, and HER2 positivity. [7] Luminal A remains the most common type of primary breast cancer with over $50 \%$ of total diagnosis, and possesses a good prognosis. Luminal B tumors, due 
to their positivity of HER2, are more proliferative and require more intensive therapy. [7] When tumors are ER and PR negative but retain HER2 positivity, they are simply referred to as "HER2" or a breast cancer that has "HER2 over-expression".

On the opposite side, it is possible for breast cancer to possess neither hormone receptors nor HER2 positivity (ER, PR, HER2-) and are termed "triple negative". [7] These tumors mimic basal epithelial cells and thus the triple negative subtype is often termed "basal". [7] Since there is no receptor to target, triple negative breast cancer tumors are more aggressive in growth and systemic therapy is often significantly deleterious. These tumors occur in younger patients and are associated with lower survival rates and increased relapse rates compared to luminal subtypes.[7] It is logical then that the aggressiveness of HER2 and the de-differentiation of triple negative tumors allows for enhanced metastases. The discovery of HER2 lead to better treatment modalities, and the recent discovery of checkpoint inhibition pathways seeks to do similarly.

The programmed death receptor (PD) and PD ligand-1 or -2 (PD-L1, PD-L2) is thought to play a crucial role in cancer developing immunological resistance. [8] Expressed on the surface of tumor cells, PD-L1 bind to PD (also known as B7-H1 or CD274) expressed on T-cells, B-cells, and natural killer cells. [8, 9] This interaction suppresses T-cell activities such as migration and cytotoxic substance secretion, thus allowing tumor cells to both escape immune functions. [8] PD-L1 is expressed in approximately $20 \%$ of TNBC [10] and is attributed with metastatic potential and enhanced proliferation. [11, 12] As TNBC has no ER, PR or HER2+ to target, PD-L1 has been of great interest to treat triple negative BCBM. 
Within the scope of brain tumors, patients with triple-negative and HER2+ subtypes are at higher risk for formation of brain metastases. [13]

\subsubsection{Breast Cancer Brain Metastases}

As survival from primary breast cancer tumors increase, the rate of developing brain metastases also increases. Breast cancer is the second most common cause of brain metastases, with breast cancer subtype being an important prognostic factor. The basal or triple negative subtype causes $>25 \%$ of all breast cancer brain metastases (BCBM), closely followed by HER2 at approximately $20 \%$. [14] It is approximated that up to $50 \%$ of all patients diagnosed with HER2 or triple negative breast cancer will eventually develop brain metastases. [15] Treatment for BCBM is restricted to surgical resection, whole brain radiation therapy, stereotactic radiosurgery, and depending on subtype, either targeted chemotherapy or nonspecific chemotherapy. [13,14] Surgical resection is performed via a minimal craniotomy and endoscope, often with patients still awake but mildly sedated to monitor integrity of brain functions. [16] Due to the invasiveness of the procedure, surgical resection is limited to fewer than 3-4 tumors that are large and symptomatic. [17]

Whole brain radiation therapy (WBRT) is a key therapy option for brain metastases in general, with typical doses of 30 Gy in 10 fractions to 37.5 Gy in 15 fractions producing significant tumor shrinkage and local disease control. [18] WBRT can cause significant toxicities such as neurocognitive decline, cataracts, blindness, and radiation necrosis. [18] For these reasons, stereotactic radiosurgery (SRS) has been promoted as the preferred radiation therapy. SRS is more precise, using multiple beams of smaller radiation doses that focus on an area to deliver a cumulative dose. This allows 
a higher dose per fraction to be administered, leading to better local control rates. [18] Despite its reduced neurocognitive decline advantage, SRS is typically only used when there are fewer than 4 brain metastases, and studies have shown that survival was not superior compared to WBRT, possibly due to lower control of total brain metastatic burden. [18] While neurosurgery and radiation techniques are slowly advancing, there is an increasing trend to improve brain penetration of existing chemotherapy and to create new agents that bypass the blood-brain and blood-tumor barriers.

\subsubsection{Conventional Breast Cancer Brain Metastases Chemotherapy}

Due to limited chemotherapeutic penetration through the blood-tumor and bloodbrain barrier and poor survival statistics, clinical trials have typically excluded patients with brain metastases. There are currently no FDA-approved medications specifically indicated for brain metastases, though temozolomide and bevacizumab are often backbone agents to treating these lesions, and a PCV (procarbazine, lomustine, and vincristine) combination is a listed option by the National Cancer Institute. [19] Typical systemic therapy includes taxanes (docetaxel, paclitaxel), anthracyclines (doxorubicin), platinum compounds (cisplatin), and alkylating agents (cyclophosphamide), often in combination with other agents. [20-23] These systemic agents were given for total tumor burden control are not intended to treat BCBM specifically.

Various combinations of cisplatin, etoposide, doxorubicin, 5-fluorouracil, methotrexate, vincristine, teniposide, lomustine, irinotecan, gemcitabine, paclitaxel, and temozolomide have been trialed in BCBM patients. The success of cisplatin has propelled the agent as part of standard non-targeted backbone therapy in combination with other agents. Cisplatin and etoposide were tested as combination agents in a Phase II trial of 4 
BCBM patients in 1997. Only 1 patient had a partial response, leading authors to conclude that though cisplatin can penetrate through the blood-tumor barrier albeit poorly. [24] Cisplatin and etoposide again were tested as combination agents in 56 BCBM patients. 7 patients achieved a "complete" response, 14 achieved partial response, 12 had no change, 16 continued to progress, and 8 patients were deemed as insufficient treatment or not assessed. An overall response rate of 38\% was noted.[25] Cisplatin, doxorubicin, 5-fluorouracil, methotrexate regimens were given to 4 patients with BCBM. However, no overall survival improvement was noted, and patients experienced major toxicity. [26]

Cyclophosphamide, methotrexate, 5-fluorouracil, doxorubicin were tried in 56 BCBM patients. The authors reported no significant prolonging of intracranial metastases free interval, but progression free survival was prolonged. [27] 15 patients with BCBM were treated with cyclophosphamide and cisplatin in a Phase II trial. Of these 15, 6 achieved partial response, defined as at least 50\% reduction in tumor, and had no new lesions or progression. [28] In another Phase II trial, 25 patients with BCBM treated with cisplatin and vinorelbine alongside $30 \mathrm{~Gy}$ of fractionated radiation. This therapy resulted in complete response in 3 patients and partial response in 16 patients, for a total response rate of 76\%. [29] The currently ongoing A-PLUS trial evaluates the BEEP (bevacizumab followed by etoposide and cisplatin) regimen alongside WBRT in BCBM patients. [NCT02185352] The BEEP combination was previously featured in a Taiwanese trial of BCBM, though no results are posted. [NCT01281696] Similarly, in a Phase II trial, 35 patients with BCBM were pretreated with bevacizumab and then given cisplatin and etoposide, with a 77\% response rate.[30] Cisplatin is also being evaluated alongside 
veliparib in the treatment of BRCA-mutated breast cancer and its associated brain metastasis. [NCT02595905]

Paclitaxel is known to have low brain and CSF concentrations. In 152 metastatic breast cancer patients with brain metastases, 78 patients responded to paclitaxel, while 6 developed CNS progression. The brain was thought to be sanctuary site for BCBM. [31] Of 4 BCBM patients given concomitant paclitaxel and bevacizumab, 3 showed partial response and 1 showed complete response, with no extra progression. [32] Five BCBM patients receiving similar bevacizumab and paclitaxel treatment showed partial response (2 patients), stable disease (2 patients), and progression (1 patient). [33]

Temozolomide as a single agent has been studied in a Phase II trial of BCBM, though no results have been posted. [NCT00831545] In combination with vinorelbine, temozolomide was used in $6 \mathrm{BCBM}$ patients to achieve only a minor response, which then progressed. [34] It was then again used in a Phase II trial of 11 BCBM patients. One had a minor response, while the others were grouped together in stable or progressing disease. [35]

Though irinotecan is not typically used to treat brain metastases, it has found utility in combination therapy. It was studied in combination with temozolomide in BCBM [NCT00617539], but results of the study are not posted. Irinotecan was combined with iniparib and showed clinical benefit in $27 \%$ of patients. [36] Similarly, eribulin is used as salvage therapy in the treatment of BCBM; as such only case reports or case series of eribulin efficacy in this setting exist. In one patient, eribulin was fifth-line initiated. One month later, brain metastases reduced. [37] In three patients with BCBM (one of each subtype), eribulin was found to be beneficial in heavily treated $n=3$ patients. 
[38] With concurrent WBRT, eribulin showed regression of one HER2- and one HER2+

BM. [39] Based on positive outcomes, eribulin is being evaluated in a Phase II trial of women with BCBM. [NCT02581839]

\subsubsection{Targeted Breast Cancer Brain Metastases Chemotherapy}

Luminal A, Luminal B, and HER2+ BCBM can be treated with targeted chemotherapy, while the triple negative subtype by definition cannot. The Luminal subtypes are often treated with selective ER modulators (SERM) such as tamoxifen, aromatase inhibitors such as anastrozole, or ER degraders such as fulvestrant, but there is no specific FDA-approved chemotherapeutic agent or regimen. [40, 41] Temozolomide has been studied in combination with WBRT, irinotecan, and capecitabine in hormone positive BCBM. [40] Upcoming therapy for hormone positive BCBM includes cyclindependent kinase 4/6 inhibitors such as abemaciclib, palbociclib [NCT02774681], and ribociclib; PI3K inhibitors such as buparlisib and pictilisib; and tyrosine kinase inhibitors such as cabozatinib. Due to the favorable prognosis of Luminal BCBM, focus has shifted to trials and chemotherapy involving the HER2+ receptor and downstream pathways, which is associated with aggressive tumors and a large percentage of BCBM.

The discovery of the HER2 receptor led to the creation of the targeted monoclonal antibody Herceptin (trastuzumab). FDA-approved in 1998, trastuzumab displayed a modest increase in overall survival. [42] Unfortunately, it was discovered that approximately $70 \%$ of patients experienced disease progression or developed metastasis within a year of starting trastuzumab. [42] Due to its size, trastuzumab is unable to penetrate the $\mathrm{BBB}$ or $\mathrm{BTB}$ in adequate concentrations, leading to the formation and inadequate control of intracranial metastases. $[4,43]$ The efficacy of the trastuzumab and 
emtansine conjugate in HER2+ BCBM is discussed in the following immunotherapy section.

The second HER2 antibody, pertuzumab, is often given in combination with trastuzumab to treat metastatic breast cancer. Case reports [44] and a subset of patients in the Phase III CLEOPATRA trial show that the combination of pertuzumab, trastuzumab, and docetaxel delays the onset of CNS metastases - but not the incidence of formationcompared to placebo, trastuzumab, and docetaxel therapy. [45] The third HER2 antibody margetuximab is currently in Phase III trials in combination with chemotherapy for the treatment of metastatic breast cancer. [46]

Tykerb (lapatinib) is a small-molecule tyrosine kinase inhibitor acting intracellularly to inhibit the HER2 signaling pathway. Unfortunately, much like trastuzumab, it does not display much efficacy in treating HER2 BCBM as monotherapy. A Phase II trial of 242 patients revealed an objective response of just $6 \%$, with $21 \%$ patients displaying a $\geq 20 \%$ volumetric lesion reduction. [47] Lapatinib is being explored in combination with other medications, most frequently capecitabine, and treatment modalities. A Phase II study in combined lapatinib and capecitabine therapy, where 29 of 44 patients achieved a partial response, though progression occurred in almost all patients and required brain radiation. [48] Many other trials have shown efficacy of lapatinib and capecitabine. [47, 49] Lapatinib was unfortunately shown not to work in combination with topotecan therapy. [50] Recently, a Phase II trial is examining the effects of lapatinib and WBRT compared to WBRT alone for women with HER2+ BCBM. [RTOG1119] 
Neratinib, another tyrosine kinase inhibitor of the HER2 pathway, was shown to be effective in treating HER2+ BCBM in combination with paclitaxel. [51] Another clinical trial combined neratinib and capecitabine to treat HER2 BCBM, with a 63\% 12month survival rate in 39 patients, though 6 patients had to discontinue due to toxicity.[52] [NCT01494662] In contrast, the HER2+ tyrosine kinase inhibitor afatinib was compared against monotherapy, combined therapy with vinorelbine, and physician's choice therapy in HER2+ BCBM. No improved efficacy in afatanib-containing regimens compared to physician's choice was noted. [53]

\subsubsection{Immunotherapy for Breast Cancer Brain Metastases}

The first immunotherapy to treat cancer was interleukin-2 (IL-2) in advanced melanoma and renal cell carcinoma. [54] In consideration of brain metastases, IL-2 had a $18.5 \%$ clinical response in previously treated patients and a $5.6 \%$ response rate in treatment-naïve patients, with only 2 of 36 naïve patients demonstrating objective intracranial disease regression. [54] IL-2 was followed by Yervoy (ipilimumab), a cytotoxic T-lymphocyte associated protein (CTLA)-4 (CD152) antibody inhibitor. In a Phase II trial, ipilimumab showed control of brain lesions in 14 of 72 metastatic melanoma patients. [55] Subsequent trials show marginal or equivocal results with combination therapy or surgical treatment. [56]

Great interest has been placed on immunotherapy in treating brain metastases. This is because unlike conventional chemotherapy, immunotherapy does not act directly on tumor cells: rather, T-cell activation leads to clinical responses seen. [57] As expected, initial trials of immunotherapy specifically precluded the enrollment of patients with 
brain metastases without specific prerequisites. [58] Immunotherapy is now being explored in the treatment of brain metastases from various cancer types.

The expression of PD-L1 on BCBM tumor surfaces is highly variable. A series of 252 brain metastases from melanoma, breast, renal, colorectal, and non-small cell, and small-cell lung cancer were analyzed for expression of PD-L1 with less than $10 \%$ showing expression. [59] In contrast, another study of 84 patients with matching primary breast tumor and brain metastases found 53\% PD-L1 expression on brain metastases. [60] Interest in immunotherapy to treat breast cancer lies especially in the treatment of TNBC, as no targets naturally exist against this subtype. [10,61] Additionally, it has been shown that TNBC may harbor elevated levels of PD-L1. [10]

Nivolumab, an antibody against PD-1, was shown to have a modest response (22\%) in a small 50-patient trial of TNBC patients. [62] Nivolumab is currently involved in a Phase II trial of metastatic TNBC brain metastases in asymptomatic and steroid-free patients. [NCT02499367] It is also involved in combination therapy for metastatic TNBC [NCT02499367], but is not being trialed specifically for brain metastases.

Pembrolizumab, another PD-1 inhibitor, was given to 111 patients with TNBC in the KEYNOTE-012 trial. Of note, 58.6\% of patients had positive expression of PD-L1. Of 27 evaluatable patients, the overall response rate was 18.5\%. [63] The KEYNOTE086 trial of pembrolizumab monotherapy in treating metastatic TNBC showed its efficacy, but again excluded any patients with brain metastases. [NCT02447003] Pembrolizumab is now being explored in the treatment of $\mathrm{BCBM}$ with the caveat of excluding HER2+ brain metastases. [NCT02886585]. In a newly launched trial, the combination of activated T-cells and pembrolizumab to treat metastatic breast cancer 
allows for previously treated, stable HER2+ BCBM patients be enrolled. [NCT03272334]

The PD-L1 inhibitor atezolizumab showed variable efficacy in the Phase I TONIC trial of 112 metastatic TNBC patients. While only $10 \%$ of patients responded to atezolizumab, the median duration response was 21 months, and all patients who responded were alive after 2 years compared to just $11 \%$ survival in non-responders after 2 years. [64] Another PD-L1 inhibitor avelumab showed effectiveness in the JAVELIN trial featuring TNBC patients, but did not look at patients with brain metastases. [65] Durvalumab is another PD-L1 inhibitor being explored for metastatic TNBC. [61] Pembrolizumab and atezolizumab are also being explored for the treatment of hormone receptor positive breast cancer. [66]

The conjugation of trastuzumab and emtansine (T-DM1) allows for specific targeting and selective cytotoxicity against HER2+ cancer cells. The discovery of T-DM1 efficacy in HER2+ BCBM was surprising, as it is even larger than the parent antibody. In early 2015, the phase III EMILIA trial compared T-DM1 against capecitabine and lapatinib in the treatment of HER2+ BCBM. Overall survival was 26.8 months in the TDM1 group and 12.9 months in the conventional treatment group. [67] Later that year, a case series of 10 patients with HER2+ breast cancer and brain metastases who received T-DM1 showed 3 patients with partial remissions and stable disease for 6 months in 2 patients, with a median survival of more than 8.5 months. [68] Multiple case series have reported reductions in intracranial lesion size with T-DM1 therapy in HER2+ BCBM. $[69,70]$ It was postulated that the antibody conjugate was better able to cross the disrupted BBB seen in tumor environments, though this remains highly controversial. 
One retrospective study suggests that T-DM1 therapy, much like trastuzumab therapy, may lead to formation of brain metastases despite adequate extracranial disease control. [71] T-DM1 was tested in the BIRTH trial, and is currently involved in the KIARA trial, both specifically for HER2+ BCBM. [NCT02135159, NCT03203616] It is being investigated in combination with tucatinib (ONT-380) for the treatment of HER2+ BCBM. [NCT01983501]

The discovery of PD-1/PD-L1 and efficacy of T-DM1 has renewed interest in exploring the microenvironment of BCBM.

\subsection{Lung Cancer Epidemiology}

In the United States, lung cancer is the second most common cause of cancer in men and women, but is the most common cause of cancer death.[72] It is estimated that more than 200,000 new cases of lung cancer will be diagnosed and more than 150,000 deaths from lung cancer will occur-more than $25 \%$ of all cancer deaths. [72]

Worldwide, it is the most common cause of cancer and of cancer death. The median age of diagnosis is at 70, with median age of death at 72. [73] The incidence of lung cancer has sharply declined for men and slowly declined for women reflecting new trends in tobacco use. Despite incidence decreasing due to initiatives to reduce tobacco use, there is approximately a 20-year lag in mortality improvement: the 5-year survival for lung cancer is only $18 \%$. This is because more than half of lung cancer will be metastatic on diagnosis due to the lack of a ubiquitous non-invasive screening tool. [72] Screening for lung cancer is limited to current or former smokers with at least a 30-pack year history.

Prior to the 1980s, lung cancer was primarily a disease of men. As women began to consume tobacco, the "gender gap" of incidence and death rates due to lung cancer 
between men and women have narrowed. [74] Women are potentially more susceptible to developing lung cancer while men have a higher mortality rate. [75] This may be due to the estrogen receptor $\beta$, which when activated causes proliferation and contributes significantly to mortality, or other genetic differences allowing for DNA damage to have greater impact than in males. [74] Smoking history remains the most important risk factor for developing lung cancer, linked to more than $80 \%$ of lung cancer cases and deaths. [74] Approximately $21 \%$ of Americans (46 million people) consume tobacco products. [73] Smoking rates and linked lung cancer rates have been declining in the United States, but rising sharply in Asian countries which huge increases in smoking rates. [73]

With smoking such a strong link to smoking to the point of being a causative factor, it is paradoxical that up to $25 \%$ of lung cancer occur in "never-smokers", which are defined as people who have smoked fewer than 100 cigarettes in their lifetime. [73] This finding is geographical, as a Japanese study showed that the percentage of neversmokers with lung cancer increased from $16 \%$ to $33 \%$ in a 30 -year period, while in the same period only $20 \%$ of lung cancer were associated with never-smokers. [73] Though smoking is postulated as a broad, causative factor for lung cancer, only specific subtypes of lung cancer are linked with smoking exposure.

\subsubsection{Lung Cancer Subtypes}

Lung cancer is split into two broad classifications: small-cell lung cancer (SCLC) and non-small cell lung cancer (NSCLC). NSCLC is responsible for more than $80 \%$ of lung cancer diagnosis. [76] Within NSCLC, the three most common subtypes are adenocarcinoma, squamous cell carcinoma, and large-cell carcinoma. These cancers form approximately $39 \%, 20 \%$ and $3 \%$ of all lung cancers, respectively. $[73,77]$ Smoking is 
strongly linked with SCLC and squamous cell carcinoma. Though adenocarcinoma is more closely linked with never-smokers, the incidence of adenocarcinoma in patients who smoke is becoming more common. [73]

SCLC is a highly aggressive tumor, possessing rapid growth, neuroendocrine activity creating paraneoplastic syndromes, high vascularity, and rapid lethality due to early metastatic dissemination. [78] Though SCLC is highly responsive to both radiation and chemotherapeutic treatment, resistance rapidly and inevitably occurs, leading to untreatable relapses. [78] Nearly ubiquitous mutations in TP53 renders genomic instability and the MYC oncogene promotes rapid growth. [78] Since SCLC typically does not possess any mutations explicitly for drug targeting, systemic chemotherapy is given by default. Due to the metastatic nature of SCLC, resection is generally not performed, in contrast to NSCLC. Patients with non-Stage IV NSCLC typically have tumor resection via video-assisted thorascopic surgery (VATS) if surgery is tolerable, followed by radiation and/or chemotherapy. [77]

Within NSCLC, adenocarcinoma possesses several mutations that influence prognosis and survival. These derangements are checked for on initial diagnosis of advanced disease. [79] Mutations in the KRAS oncogene occurs in up to $25 \%$ of adenocarcinoma and is associated with smoking history. Presence of KRAS mutations increases tumor aggressiveness and disease relapse, along with reduced response or increased resistance to chemotherapy. [80] Interestingly, KRAS mutations have an inconsistent effect on prognosis, as trials have reported that both wild type and mutated $K R A S$ have similar outcomes on chemotherapy or that mutants have shorter progressionfree and overall survival. [81] Mutations in $K R A S$ may be a poor prognosis marker 
specifically in women with stage I NSCLC and Asian patients, and mutations in codon 13 confers resistance to chemotherapy when compared to codon 12 mutations. [81] KRAS acts downstream from the EGFR pathway, thus mutations in the KRAS oncogene lead to suboptimal therapeutic response or outright resistance to EGFR inhibitors. [82]

Mutations of EGFR occur in approximately $20 \%$ of adenocarcinoma, which allows the evasion of programmed cell death, enhanced migration, and metastases facilitation. [83] Found in women, never-smokers and Asian patients, EGFR mutations however confer a positive prognosis, as the receptor is susceptible to inhibition by competitive tyrosine kinases. [83, 84] The most common EGFR mutations occur on exons 18-21, with exon 19 deletion (L8658R) being the most common (60\%) and a point mutation on exon 20 being second-most common. [83, 85] It is purported that the mutations on exons 19 and 21 allow for targeting of tyrosine kinase inhibitors. [86] Insertions on exon 20 may lead to tyrosine kinase inhibitor resistance, and the T790M mutation is found in more than half of treated tumors. [83] While EGFR mutation occur early in disease, amplification occurs late in disease and is associated with metastases. [87] Inhibitors to overcome this mutation have been developed, which in turn have caused a C797 mutation to develop within the EGFR ATP-binding site. Unfortunately, targeted inhibitors do not improve survival without an EGFR mutation, so systemic chemotherapy must be given to patients with wild-type EGFR. [85]

The discovery of anaplastic lymphoma kinase $(A L K)$ and echinoderm microtubule-associated protein-like 4 (EML4) rearrangements which occur in $7 \%$ of adenocarcinoma in 2007 was monumental and drove tyrosine kinase inhibitor development. [88] The t(2;5) chromosomal translocation allows constitutive $A L K$ 
activity. [85] ALK-EML4 mutations are found in never-smokers and younger patients, similar to EGFR mutations, though mutually exclusive and distant from both and $K R A S$ mutations. [89] Of 22 potential translocation variants, three major variants of $A L K$ fusion are found clinically. Variant 1 features a E13;A20 exon fusion (33\% of total $A L K$ mutations), variant 3 has a E6a/b;A20 fusion (29\%), and variant 2 possesses a E20;A20 fusion (9\%). [88] One small trial of 54 patients discovered that possession of the $3 \mathrm{a} / \mathrm{b}$ $A L K$ variant was a source of inhibitor resistance, but other trials report no correlation of $A L K$ variance type on survival. $[90,91]$ Possession of an $A L K$ translocation or mutation is associated with a poor prognosis and relapse or resistance to drugs within the first year of treatment. [92]

Approximately $2 \%$ of lung cancer have $R O S-1$ fusion mutations. Much like $A L K$ EML4 translocation mutations, $R O S-1$ mutations are associated with younger age and never-smokers. [93] Unlike the $A L K$-EML4 fusion protein, $R O S$ - 1 fusion protein mechanisms are unknown, but often feeds into common tyrosine kinase-signaling pathways. [94] Since it is so uncommon, ROS-1 testing is often performed only after EGFR and $A L K$ results are negative. [94] The oncogene initially became an object of interest when it was discovered it was concomitantly inhibited by the first $A L K$ inhibitor crizotinib, which subsequently gained FDA-approval to treat ROS-1 rearrangement NSCLC. [94] $A L K$ and ROS- 1 are so similar that more than $75 \%$ of amino acids are similar within the respective ATP-binding sites, which may explain why crizotinib is so effective in treating ROS-1 mutated NSCLC. [95] Newer $A L K$ inhibitors such as ceritinib, lorlatinib, and entrectinib also inhibit $R O S$-1, presumably due to the homology. [96] 
The expression of PD-L1 in NSCLC has been a subject of debate since variable expression exists not only between patients, but within tumor samples across time as treatment occur. A study of 95 patients showed that PD-L1 expression might increase when treated with EGFR inhibitors, thus enhancing the effects of PD-1/PD-L1 therapy. [97] Another trial of four patients showed that PD-L1 expression changed after chemotherapy and inhibitor treatment. [98] Though the presence of high levels of PD-L1 is a poor prognosis indicator, especially in NSCLC, the presence allows for treatment with PD-1/PD-L1 inhibitors, which have revolutionized treatment for many cancer types. $[99,100]$ As the check-and-balance process of the immune system is impaired to allow hyperactivity, PD-1/PD-L1 inhibitors create significant burdensome and severe toxicity. [101] Despite these drawbacks, inhibitors are becoming a frontline therapy for the treatment of NSCLC and many solid tumors.

Within the scope of brain metastases, EGFR mutations and $A L K$ or $R O S-1$ translocations allow targeting via tyrosine kinase inhibitors. First-generation inhibitors poorly penetrate the blood-brain or blood-tumor barrier and cause relapse within a year. [102] Subsequent generations are more effective and are being developed with the intent to treat brain metastases. [103]

\subsubsection{Lung Cancer Brain Metastasis}

More than $50 \%$ of all brain metastases are caused by lung cancer and the most common place for lung cancer to metastasize is the brain. [104] SCLC is the most likely lung cancer subtype to metastasize to the brain; at diagnosis approximately $20 \%$ of patients with SCLC will already have brain metastases, and approximately $80 \%$ will develop brain metastases within 2 years. $[105,106]$ In contrast, approximately $7 \%$ of 
NSCLC patients will have brain metastases on presentation and up to $45 \%$ will develop brain metastases within 2 years. $[105,107,108]$ Mutation status may play an important role in the development of lung cancer brain metastases (LCBM). It was found that mutant $E G F R$ was more likely to develop multiple brain metastases than wild-type, and exon 19 deletions had smaller brain metastases. $[109,110]$ Approximately $30 \%$ of $A L K$ NSCLC patients will develop brain metastases. [111] Conversely, ROS-1 mutations may cause lowered brain metastases rates. [111] Median survival of patients with LCBM at presentation is approximately 4 months, and with aggressive treatment lengthens to just over 12 months. [108, 112, 113] Patients who develop brain metastases later in disease tend to live longer than those at diagnosis, and receiving chemotherapy increases survival by approximately 2 months. [112]

Much like BCBM, chemotherapy, surgery, and radiation are components in firstline treatment of LCBM. Karnofsky performance status (KPS), age, number of intracranial metastases, control of primary cancer, and the presence of extracranial metastases are factors that predict clinical outcomes. [114] Female gender, age less than 58, adenocarcinoma subtype, single brain lesions, and surgical resection are considered to be favorable prognostic factors. [115] Surgery is typically restricted to large and symptomatic metastases. In the Quartz trial, WBRT revealed no survival benefit in the context of LCBM. [116] Additionally, WBRT causes significant neurotoxicity and cognitive decline. SRS shows a better adverse effect profile, and when combined with WBRT, shows significant tumor control with lower rates of new intracranial lesions. [117] Chemotherapy is often given to control primary tumor, but due to the failure to 
adequately cross the blood-brain and blood-tumor barriers, often results in subtherapeutic activities in treating LCBM.[108]

\subsubsection{Conventional Lung Cancer Brain Metastases Chemotherapy}

Brain metastases from both SCLC and NSCLC have a history of responsiveness to chemotherapy. SCLC brain metastases are more initially responsive and often have a complete response, followed by relapse. [118] Brain metastases from NSCLC that possess a mutation surprisingly have a favorable outcome and responsiveness to chemotherapy, perhaps due to the necessity of the oncogene to exert tumor activity. [119] The backbone of most non-targeted lung cancer therapy consists of cisplatin and another agent such as etoposide, topotecan, or pemetrexed. [108, 120, 121] Temozolomide is commonly added to treat brain lesions. Other drugs to treat LCBM include vincristine, cyclophosphamide, lomustine, and doxorubicin. [113]

In the GFPC 07-01 Phase II trial, 43 NSCLC brain metastases patients were treated with cisplatin and pemetrexed every 3 weeks, in combination with WBRT. Survival was extended to 7.4 months, with the development of severe hematological toxicity in 17 patients. [122] Another similar trial with cisplatin, pemetrexed, and radiation extended survival to 12.6 months with combination therapy. [123] In a comparison of cisplatin and pemetrexed against temozolomide and pemetrexed, it was found that the same efficacy existed but pemetrexed and temozolomide had fewer adverse reactions, making the combination potentially more beneficial for NSCLC brain metastases. [124] Since pemetrexed achieves CSF concentrations similar to serum levels, it has been described as the "preferred" agent in platinum doublet chemotherapy. [125] 
Bevacizumab has gained footing as a standard-of-care therapy option in the treatment of LCBM. Initial concern over bevacizumab administration in brain metastases due to the risk of intracranial hemorrhage was mitigated as two trials showed its safety in this setting. [126] In the French EOLE cohort study, bevacizumab alongside platinumdoublet therapy showed similar survival in patients with or without brain metastases. [127]. In a case series of 4 NSCLC patients with a total of 60 brain metastases, bevacizumab was combined cisplatin and pemetrexed along with stereotactic radiosurgery, and was found to reduce the volume of tumors by an average of $75 \%$. [128] More clinical trials have supported the use of bevacizumab and platinum-based chemotherapy in the treatment of NSCLC brain metastases. [128-133] Presence of brain metastases is advocated to no longer be a contraindication to bevacizumab use. [134]

Temozolomide, FDA-approved for glioblastoma, has been explored for treating LCBM. Results are varied: temozolomide was not shown to have any efficacy in the Phase II EORTC 08965 study [135], and surprisingly not even in combination with radiation therapy shows efficacy. [136] In contrast, another Phase II trial showed stable disease in $10 \%$ of patients treated with temozolomide previously treated with WBRT and chemotherapy.[137] Two clinical trials involving temozolomide specifically for NSCLC brain metastases have been conducted, but no results are posted. [NCT00076856, NCT00034697] Currently, temozolomide does not play an active role in first-line therapy for LCBM.

\subsubsection{Targeted Lung Cancer Brain Metastases Chemotherapy}

Due to the presence of numerous genetic aberrations, targeted inhibitors are being explored for concomitant primary lung tumor tumor and accompanying brain metastases 
treatment. Three major therapeutic targets exist: EGFR, $A L K$, and PD/PD-L1. The latter will be explored in the immunotherapy subsection. Beginning with the first-generation EGFR inhibitors in 2004, gefitinib and erlotinib were the first step in personalized medication for NSCLC. Due to the high presence of brain metastases in NSCLC patients, gefitinib and erlotinib were tested for efficacy in this setting shortly after discovery.

Gefitinib is the first of the first-generation EGFR inhibitors approved for NSCLC patients. It has been shown to penetrate through the BBB, though the extent is controversial. Plasma-to-CSF ratios is reported as approximately $20 \%$ [138], with a strong dose correlation. [139] Human pharmacokinetics apparently vastly differ from primate kinetic data, which show virtually no gefitinib uptake into brain parenchyma. [138] Multiple small trials evaluated gefitinib in EGFR-mutated LCBM, both concluded gefitinib provides enhanced survival. [140-142] Gefitinib has also shown efficacy when combined with WBRT. [139] One case report shows gefitinib treating LCBM refractory to erlotinib therapy, which is discussed next. [143]

Erlotinib has been shown to penetrate the BBB ease, achieving concentrations in the CSF required to reduce intracranial tumor burden. [144] PET/CT scans show erlotinib accumulating preferentially in brain lesions compared to normal brain parenchyma. [145] A 69-patient trial showed erlotinib achieving an $82.4 \%$ response rate in patients with EGFR mutant LCBM. [146] In one instance, erlotinib was able to control a brain metastases refractory to another $E G F R$ inhibitor afatinib, a second generation $E G F R$ inhibitor, discussed next. [147]

Differences among the first generation EGFR inhibitors exist. A comparison of 119 gefitinib and 13 erlotinib patients with NSCLC brain metastases showed erlotinib 
having both better intracranial disease control and lower recurrence rate. [148]

Unfortunately, both gefitinib and erlotinib require $E G F R$ mutations in order to be active, and Phase II trials demonstrated no beneficial effects when these agents were given in EGFR wildtype LCBM patients. $[149,150]$ Another trial confirmed these findings in combination with WBRT. [151] The role of first-generation EGFR inhibitors and radiation therapy is not clear-cut. In instances, erlotinib has been shown to be synergistic with WBRT [152], while in other cases there was no significant improvement in combination. [153]

Afatinib, a member of the second-generation EGFR inhibitors, was shown to be effective in treating EGFR-mutated LCBM during the LUX-Lung 3 and LUX-Lung 6 trials compared to cisplatin-pemetrexed or cisplatin-gemcitabine therapy, respectively. [154] Patients in these trials respondent to afatinib had an exon 19 deletion and L858R point mutation. This finding was peculiar, as preclinical work suggested afatinib is a substrate for drug efflux pumps such as P-glycoprotein and BCRP, restricting accumulation into the brain. [155] On the other hand, a LCBM model using PC-9 EGFR mutant cells showed penetration of the BBB and a reduction in tumor burden. [156] The discordance of preclinical and clinical finding has led to afatinib being involved in a pharmacokinetic study to determine distribution and dose-responses in LCBM. [NCT02423525] Dacomitinib, another member of the second-generation irreversible EGFR inhibitors, was being explored for brain metastases, though the study is now terminated. [NCT02047747]

Despite these advances in targeted therapy and increasing BBB/BTB penetration, CNS progression occurs in approximately $50 \%$ of patients within 3 years of therapy 
initiation. [157] Due to the high incidence of lung cancer patients developing brain metastases, focus shifted on creating targeted inhibitors to treat LCBM.

Osimertinib is currently the only FDA-approved member of the third generation EGFR-inhibitors. While it also targets exon 19 deletions and L858R mutations, it is specifically used when first- or second-generation EGFR inhibitors have failed and there is a concomitant T790M mutation. [158] The prevalence and significance of the mutation is discussed in the subtype section, though one study has noted the presence of T790M mutations in brain metastases is lower than that of primary lung disease. [159] In the AURA (extension) and AURA2 trial, 50 patients with asymptomatic brain metastases were treated with osimertinib. 27 of 50 showed overall improvement and 6 of 50 showed complete CNS response. [160] In the Phase III AURA3 trial, osimertinib was superior to chemotherapy in treating T790M LCBM. [161] The AURA17 trial similarly showed osimertinib leading to complete response in 10 patients and partial response in 15 patients with T790M LCBM. [NCT02442349] The upcoming Phase II APPLE trial will compare the effect of osimertinib and gefinitib on LCBM. [162] The upcoming third-generation AZD3759 is being trialed against osimertinib in the BLOOM study, which specifically focuses on LCBM efficacy. [163] [NCT02228369]

$A L K$ inhibitor discovery have outpaced that of EGFR inhibitors, perhaps due to their concomitant $R O S-1$ inhibition. Three generations of inhibitors exist, and much like EGFR inhibitors, increases in generation are correlated with enhanced BBB/BTB penetration for intracranial efficacy.[164]

The first agent FDA-approved for treating $A L K$-rearranged NSCLC was crizotinib. Though good at controlling peripheral disease, a case report showed CSF 
accumulation and subsequent CNS disease progression. [165] Pooled data from the PROFILE 1005 and PROFILE 1007 trials showed that CNS progression occurred in 70\% of patients treated with crizotinib. [166] Crizotinib does initially control intracranial disease, but progression is inevitable, with most patients developing resistance within 2 years. [167] It is postulated that the inefficacy in LCBM is due to efflux pump activity, as crizotinib is a substrate for ABCB1/ABCG2. [168] Similar efflux pumps restrict full ceritinib penetration into the brain, but apparently not its efficacy in LCBM. [169]

Ceritinib, a member of the second-generation $A L K$ inhibitors, is used in patients who have progressed or become resistant on crizotinib therapy. Results of the ASCEND1 trial showed ceritinib controlled intracranial disease in 15 of 19 treatment-naïve LCBM patients and 49 of 75 pretreated LCBM patients. [170] This led to the Phase II ASCEND2 trial which confirmed the ASCEND-1 findings of ceritinib use in both crizotinibpretreated and chemotherapy-naïve patients with $A L K$-mutated LCBM. [171, 172] The recently-conducted ASCEND-5 trial compared single-agent ceritinib against chemotherapy in patients who had progressed on both crizotinib and platinum-based chemotherapy. Preliminary results show ceritinib improving overall survival. [173]

The other second-generation $A L K$ inhibitor, alectinib, is a poor substrate for efflux pumps such as ABCB1 and ABCG2. [174] In a Phase II study, 84 crizotinib-refractory LCBM patients were treated with alectinib. $83 \%$ of CNS disease rate was controlled for a median of 10.3 months, though at 12 months $24.8 \%$ had CNS progression. [175] Two recent trials have shown alectinib's efficacy in LCBM. The Phase III ALEX trial compared alectinib and crizotinib with a radiation modifier, showing that alectinib 
achieves response with or without radiation [176], and the ALUR trial showed alectinib superiority over platinum-doublet therapy in treating brain metastases. [177]

Brigatinib, a combined third-generation $A L K$ and $E G F R$ inhibitor, was approved in April 2017 for the treatment of metastatic NSCLC, specifically as second-line therapy for ALK-positive NSCLC. In the first of two Phase II trials, 31 patients (35\%) had complete resolution of brain lesions, while in the second study, a combined $25 \%$ had complete resolution of brain lesions. [178, 179]

Lorlatinib, a combined $A L K$ and $R O S-1$ inhibitor, is undergoing clinical phase trials for the treatment of $A L K$-positive NSCLC. It is said that lorlatinib was designed to primarily permeate through the BTB and treat LCBM. [180] ${ }^{11} \mathrm{C}$-lorlatinib was given to non-human primates and PET imaging revealed enhanced distribution to the brain, liver, and spleen. [180]Similarly, nude mice bearing NCI-H3122 subcutaneous xenografts were given the radioactive drug and showed enhanced uptake. [180] In a Phase II trial of patients with $A L K$-LCBM, 7 of 23 patients had a complete response on the drug, while 4 had a partial response. [181]

Entrectinib, an $A L K, R O S-1$, and Trk A-C inhibitor, has shown to be effective in NTRK-positive LCBM. Similar to lorlatinib, it is designed to cross the BBB/BTB. [182]

Two possibilities for initial response followed by relapse is the presence of the BBB and BTB, which create a sub-therapeutic environment for resistance to form. This is the case for all chemotherapy acting directly on receptors or targets in tumor cells, but as discussed next, not a possibility for immunotherapy. 


\subsubsection{Immunotherapy for Lung Cancer Brain Metastases}

Much like breast cancer, the discovery of PD-1 and PD-L1 checkpoint inhibitors has propelled agents to first-line therapy in the treatment of lung cancer. It was found that NSCLC LCBM has higher expression of PD-L1 compared to the primary lung tumor. $[58,163]$ Further, PD-L1 expression in the context of NSCLC is associated with poor

prognosis. [183] PD-1 and PD-L1 inhibitors are typically antibodies, such as nivolumab, pembrolizumab, and atezolizumab. All three are FDA-approved to treat metastatic NSCLC after progression on platinum-based therapy. [184] Pembrolizumab can be used as first-line therapy in NSCLC patients without $E G F R$ or $A L K$ mutations, though if these mutations are present, all three agents are indicated if patients progress despite targeted therapy.

Since EGFR inhibitors upregulate the PD-L1 expression on tumor cells, trials initially recruited patients who were refractory to these agents. One study has shown that patients with wild-type EGFR show better improvement in overall survival compared to patients with EGFR mutants when given checkpoint inhibitors. [185] The role of PD1/PD-L1 inhibitors in the presence of EGFR mutation has been a subject of investigation. [186-190] Preliminary reports combined targeted agents and checkpoint inhibitors, revealing encouraging results but early termination due to significant side effects. [191, 192] Clinical trials are ongoing combining the two therapies. [NCT02039674, NCT02364609, NCT02013219, NCT02574078, NCT02393625, NCT02584634, NCT02898116, NCT02323126, NCT02454933, NCT01998126]

Interestingly, KRAS mutations are not associated with increases of tumor PD-L1 expression, and $A L K$ oncogene expression have variable effects. [184, 193] To date, no 
published data specifically combine $A L K$-inhibitors and PD-1/PD-L1 therapy in the context of metastatic NSCLC or LCBM, but trials are underway. [NCT02511184, NCT01998126]

Pembrolizumab, in the KEYNOTE-024 trial, used information from KEYNOTE001 and KEYNOTE-010 to prove that it was superior to physician's choice of platinumbased doublet therapy in the treatment of NSCLC. Enrollment included patients who had no EGFR mutations or $A L K$ translocations. [194] In a small Phase II trial with 18 NSCLC patients with brain metastases, pembrolizumab achieved a response rate in 6 patients (33\%) that was sustained in all but one patient. [195] It is being explored in the treatment of brain metastases, but excludes SCLC and NSCLC with either EGFR mutations or ALK translocations. [NCT02886585]

Nivolumab showed an improvement in 2 of 12 patients with untreated NSCLC CNS metastases in a portion of the CheckMate 012 trial. [196] In five patients with LCBM, one patient achieved a complete and partial response each. [197] A retrospective analysis of 48 LCBM patients revealed no response with nivolumab monotherapy, but did show a response with radiation therapy prior to nivolumab initiation. [196] These data lead nivolumab to be combined with stereotactic radiosurgery in an active Phase II trial of LCBM patients. [NCT02978404]

In the Phase III PACIFIC trial, durvalumab showed efficacy in patients with NSCLC. In this trial, new incidence of LCBM occurred in $11 \%$ in placebo-treated patients compared to $5.5 \%$ treated with durvalumab. [198] Capitalizing on this success, durvalumab is now being studied in patients with brain metastases from NSCLC. [NCT0266914] 
Much like the other two checkpoint inhibitors, atezolizumab too has shown efficacy in LCBM. In a subset of OAK patients, atezolizumab was compared with docetaxel on survival from, and time to develop LCBM. Atezolizumab improved survival by nearly 8 months compared to docetaxel, with a lower risk of developing new lesions. [199] Avelumab, an upcoming PD-L1 inhibitor, was shown to be effective in the JAVELIN trial for NSCLC, but did not include patients with brain metastases. [200] The upcoming JAVELIN-100 trial includes asymptomatic LCBM patients with certain criteria. [NCT02576574]

Other forms of therapy to stimulate the immune system include adoptive cell transfer of tumor-infiltrating lymphocytes, BCG vaccine, and antigen-specific cancer vaccines. These have not been shown to be effective in the treatment of NSCLC or their brain metastases. [201]

\subsection{Glioblastoma Epidemiology}

While $80 \%$ of all intracranial tumors are from metastases, the primary malignant brain tumor glioblastoma comprises approximately 16\%. [202] Glioblastomas primarily occur in the frontal or temporal regions of the cortex, though instances of tumors in the spinal cord, cerebellum, and brain stem have been noted. [202] Though highly invasive through brain parenchyma, they are restricted to these areas of the central nervous system and do not metastasize. [203] Much like other intracranial tumors, glioblastoma are highly heterogeneous and feature mutations in chromosomes leading to tumor suppressor gene loss and/or amplification and expression of oncogenes. [203] The median age of diagnosis is 64 , with a median survival of 14-16 months and a 5-year survival of $9.8 \%$. [204] 
The risk factors for developing glioblastoma are not definitively recognized, however ionizing radiation has been highly associated with the delayed development of gliomas. [202] Cellular mutations of $p 53, E G F R, P T E N, P I 3 K, R A S$, and $R b$ have been found in glioblastoma, along with isocitrate dehydrogenase 1 mutations in secondary glioblastomas. [202, 204] Interestingly, the enzyme O-6-methylguanine-DNA methyltransferase (MGMT) is a predictive factor for survival: patients with MGMTmethylated tumors survive approximately twice as long as non-MGMT-methylated tumors (22-26 months vs. 12-15 months, respectively). [204] The MGMT enzyme is associated with better outcomes from medication therapy. [202, 203]

Current therapy for glioblastoma includes surgical resection followed by adjuvant radiation therapy, often combined with chemotherapy. Aggressive surgical resection, especially with greater extent around margins, is associated with better outcomes. The size $(>5 \mathrm{~cm})$ and location of tumors (brainstem and diencephalon) are associated with poorer outcomes. [202] After healing from the surgery, radiation and chemotherapy are next. The typical radiation schedule is $60 \mathrm{~Gy}$ divided in 30 fractions, which is a lower per-dose fraction than other schedules but gives a higher total dose overall. [203] The next section talks about two FDA-approved non-specific chemotherapeutic agents for glioblastoma; no targeted agents exist.

\subsubsection{Glioblastoma Chemotherapy}

Two DNA-alkylating agents, temozolomide and carmustine, are FDA-approved for use in treating glioblastoma. [203] Temozolomide is orally administered concomitantly with radiation therapy, acting as both a cytotoxic agent and radiosensitizer. [205] It was shown that the $M G M T$ may mediate this radiosensitivity. [205] This is 
because $M G M T$ encodes a DNA-repair enzyme; methylated $M G M T$ inhibits this enzyme from being expressed and thus temozolomide activity is unimpaired. [202] Temozolomide as monotherapy improves survival to 15 months from 12 months with radiation therapy alone, with the combination of drug and radiation leading to $27 \%$ survival at 2 years and $10 \%$ at 5 years vs. $11 \%$ and $2 \%$ for radiation, respectively. [202]

Carmustine, administered as degradable wafers, are applied to the regions remaining after tumor excision. Though a Phase III trial showed survival benefit compared to placebo, carmustine toxicity and the ease of temozolomide administration precludes the wafers from use as first-line therapy to treat glioblastoma. [203]

\subsubsection{Immunotherapy for Glioblastoma}

Much like melanoma, IL-2 was one of the first immunotherapeutic agents trialed in the treatment of glioblastoma. In 9 GBM patients, IL-2 administration showing increases in survival periods ranging 7- to 17-months. [206] A vaccine against the EGFRvIII, which is displayed in 20-30\% of glioblastoma, did not increase survival in patients with newly diagnosed glioblastoma in combination with temozolomide or in comparison with temozolomide monotherapy. [207]

Checkpoint inhibition is said to "revolutionize" treatment of solid tumors such as melanoma, non-small cell lung cancer, breast cancer, cancers which make up the top 8085\% of brain metastases. In glioblastoma, the Phase III CheckMate-143 trial compared nivolumab to standard-of-care bevacizumab. No improvement in overall survival was noted. [NCT02017717] Despite this finding, nivolumab is being compared to temozolomide (each in combination with radiation therapy) [NCT02617589] and also in combination with temozolomide and radiation therapy against placebo. [NCT02667587] 
Pembrolizumab was tested in combination with bevacizumab for newly-diagnosed glioblastoma [NCT02337491], and with MRI-guided laser ablation in recurrent glioblastoma [NCT02311582]. Durvalumab is being tested in combination with radiation therapy and bevacizumab [NCT02336165].

\subsection{References}

1. National Cancer Institute, N.C.I., Breast Cancer Risk in American Women. 2012.

2. DeSantis, C.E., et al., Breast cancer statistics, 2017, racial disparity in mortality by state. CA Cancer J Clin, 2017. 67(6): p. 439-448.

3. Natali, P.G., et al., Expression of the p185 encoded by HER2 oncogene in normal and transformed human tissues. Int J Cancer, 1990. 45(3): p. 457-61.

4. Azim, H.A. and H.A. Azim, Jr., Systemic treatment of brain metastases in HER2positive breast cancer: current status and future directions. Future Oncol, 2012. 8(2): p. 135-44.

5. Mauvais-Jarvis, P., F. Kuttenn, and A. Gompel, Antiestrogen action of progesterone in breast tissue. Breast Cancer Res Treat, 1986. 8(3): p. 179-88.

6. Schnitt, S.J., Classification and prognosis of invasive breast cancer: from morphology to molecular taxonomy. Mod Pathol, 2010. 23 Suppl 2: p. S60-4.

7. Dai, X., et al., Breast cancer intrinsic subtype classification, clinical use and future trends. Am J Cancer Res, 2015. 5(10): p. 2929-43. 
8. Sheng, J., et al., Expression of programmed death ligand-1 on tumor cells varies pre and post chemotherapy in non-small cell lung cancer. Sci Rep, 2016. 6: p. 20090.

9. Kerr, K.M. and M.C. Nicolson, Non-Small Cell Lung Cancer, PD-L1, and the Pathologist. Arch Pathol Lab Med, 2016. 140(3): p. 249-54.

10. Mittendorf, E.A., et al., PD-L1 expression in triple-negative breast cancer. Cancer Immunol Res, 2014. 2(4): p. 361-70.

11. Zhang, M., et al., Expression of PD-L1 and prognosis in breast cancer: a metaanalysis. Oncotarget, 2017. 8(19): p. 31347-31354.

12. Karnik, T., et al., PD-L1 in Breast Cancer: Comparative Analysis of Three Different Antibodies. Hum Pathol, 2017.

13. Kyeong, S., et al., Subtypes of breast cancer show different spatial distributions of brain metastases. PLoS One, 2017. 12(11): p. e0188542.

14. Witzel, I., et al., Breast cancer brain metastases: biology and new clinical perspectives. Breast Cancer Res, 2016. 18(1): p. 8.

15. O'Sullivan, C.C., et al., Current challenges in the management of breast cancer brain metastases. Semin Oncol, 2017. 44(2): p. 85-100.

16. Jandial, R., et al., Operative and Therapeutic Advancements in Breast Cancer Metastases to the Brain. Clin Breast Cancer, 2017.

17. Leone, J.P. and B.A. Leone, Breast cancer brain metastases: the last frontier. Exp Hematol Oncol, 2015. 4: p. 33.

18. Willett, A., et al., Management of solitary and multiple brain metastases from breast cancer. Indian J Med Paediatr Oncol, 2015. 36(2): p. 87-93. 
19. National Cancer Institute, N.C.I., Drugs Approved for Brain Tumors. 2014.

20. Miles, D.W., et al., Phase III study of bevacizumab plus docetaxel compared with placebo plus docetaxel for the first-line treatment of human epidermal growth factor receptor 2-negative metastatic breast cancer. J Clin Oncol, 2010. 28(20): p. $3239-47$.

21. Miller, K., et al., Paclitaxel plus bevacizumab versus paclitaxel alone for metastatic breast cancer. N Engl J Med, 2007. 357(26): p. 2666-76.

22. Isakoff, S.J., et al., TBCRC009: A Multicenter Phase II Clinical Trial of Platinum Monotherapy With Biomarker Assessment in Metastatic Triple-Negative Breast Cancer. J Clin Oncol, 2015. 33(17): p. 1902-9.

23. Linot, B., et al., Use of liposomal doxorubicin-cyclophosphamide combination in breast cancer patients with brain metastases: a monocentric retrospective study. $\mathrm{J}$ Neurooncol, 2014. 117(2): p. 253-9.

24. Vinolas, N., et al., Phase II trial of cisplatinum and etoposide in brain metastases of solid tumors. J Neurooncol, 1997. 35(2): p. 145-8.

25. Franciosi, V., et al., Front-line chemotherapy with cisplatin and etoposide for patients with brain metastases from breast carcinoma, nonsmall cell lung carcinoma, or malignant melanoma: a prospective study. Cancer, 1999. 85(7): p. 1599-605.

26. Lamar, R.E., et al., High-dose, brief duration, multiagent chemotherapy for metastatic breast cancer. Cancer, 1994. 73(7): p. 1842-8.

27. Lentzsch, S., et al., Brain metastases in breast cancer: prognostic factors and management. Eur J Cancer, 1999. 35(4): p. 580-5. 
28. Christodoulou, C., et al., Temozolomide (TMZ) combined with cisplatin (CDDP) in patients with brain metastases from solid tumors: a Hellenic Cooperative Oncology Group (HeCOG) Phase II study. J Neurooncol, 2005. 71(1): p. 61-5.

29. Cassier, P.A., et al., A phase 2 trial of whole-brain radiotherapy combined with intravenous chemotherapy in patients with brain metastases from breast cancer. Cancer, 2008. 113(9): p. 2532-8.

30. Lu, Y.S., et al., Bevacizumab preconditioning followed by Etoposide and Cisplatin is highly effective in treating brain metastases of breast cancer progressing from whole-brain radiotherapy. Clin Cancer Res, 2015. 21(8): p. 1851-8.

31. Freilich, R.J., A.D. Seidman, and L.M. DeAngelis, Central nervous system progression of metastatic breast cancer in patients treated with paclitaxel. Cancer, 1995. 76(2): p. 232-6.

32. Labidi, S.I., et al., Bevacizumab and paclitaxel for breast cancer patients with central nervous system metastases: a case series. Clin Breast Cancer, 2009. 9(2): p. $118-21$.

33. Yamamoto, D., et al., Bevacizumab in the treatment of five patients with breast cancer and brain metastases: Japan Breast Cancer Research Network-07 trial. Onco Targets Ther, 2012. 5: p. 185-9.

34. Omuro, A.M., et al., Vinorelbine combined with a protracted course of temozolomide for recurrent brain metastases: a phase I trial. J Neurooncol, 2006. 78(3): p. 277-80. 
35. Iwamoto, F.M., et al., A phase II trial of vinorelbine and intensive temozolomide for patients with recurrent or progressive brain metastases. J Neurooncol, 2008. 87(1): p. $85-90$.

36. Anders, C., et al., TBCRC 018: phase II study of iniparib in combination with irinotecan to treat progressive triple negative breast cancer brain metastases. Breast Cancer Res Treat, 2014. 146(3): p. 557-66.

37. Matsuoka, H., et al., Regression of brain metastases from breast cancer with eribulin: a case report. BMC Res Notes, 2013. 6: p. 541.

38. Chang, A.Y. and X.X. Ying, Brain Metastases from Breast Cancer and Response to Treatment with Eribulin: A Case Series. Breast Cancer (Auckl), 2015. 9: p. 1924.

39. Byun, K.D., et al., Eribulin Mesylate Combined with Local Treatment for Brain Metastasis from Breast Cancer: Two Case Reports. J Breast Cancer, 2016. 19(2): p. 214-7.

40. Liu, M.C., J. Cortes, and J. O'Shaughnessy, Challenges in the treatment of hormone receptor-positive, HER2-negative metastatic breast cancer with brain metastases. Cancer Metastasis Rev, 2016. 35(2): p. 323-32.

41. Hajac, Ł., Fulvestrant endocrine therarapy in patient with CNS dissemination of breast cancer. Vol. 11. 2015.

42. Vu, T. and F.X. Claret, Trastuzumab: updated mechanisms of action and resistance in breast cancer. Front Oncol, 2012. 2: p. 62. 
43. Terrell-Hall, T.B., et al., Trastuzumab distribution in an in-vivo and in-vitro model of brain metastases of breast cancer. Oncotarget, 2017. 8(48): p. 8373483744.

44. Senda, N., et al., Pertuzumab, trastuzumab and docetaxel reduced the recurrence of brain metastasis from breast cancer: a case report. Breast Cancer, 2016. 23(2): p. 323-8.

45. Swain, S.M., et al., Incidence of central nervous system metastases in patients with HER2-positive metastatic breast cancer treated with pertuzumab, trastuzumab, and docetaxel: results from the randomized phase III study CLEOPATRA. Ann Oncol, 2014. 25(6): p. 1116-21.

46. Rugo, H.S., et al., SOPHIA: A phase 3, randomized study of margetuximab (M) plus chemotherapy (CTX) vs trastuzumab (T) plus CTX in the treatment of patients with HER2 + metastatic breast cancer $(M B C)$. Journal of Clinical Oncology, 2016. 34(15_suppl): p. TPS630-TPS630.

47. Lin, N.U., et al., Multicenter phase II study of lapatinib in patients with brain metastases from HER2-positive breast cancer. Clin Cancer Res, 2009. 15(4): p. 1452-9.

48. Markman, M., Lapatinib as a therapeutic option in brain metastases from HER2+ breast cancer. Annals of Palliative Medicine, 2013. 2(1): p. 35-36.

49. Bachelot, T., et al., Lapatinib plus capecitabine in patients with previously untreated brain metastases from HER2-positive metastatic breast cancer (LANDSCAPE): a single-group phase 2 study. Lancet Oncol, 2013. 14(1): p. 6471. 
50. Lin, N.U., et al., Randomized phase II study of lapatinib plus capecitabine or lapatinib plus topotecan for patients with HER2-positive breast cancer brain metastases. J Neurooncol, 2011. 105(3): p. 613-20.

51. Pegram, M.D., Neratinib in ERBB2-Positive Brain Metastases. JAMA Oncol, 2016. 2(12): p. 1541-1543.

52. Freedman, R.A., et al., Translational Breast Cancer Research Consortium (TBCRC) 022: A Phase II Trial of Neratinib for Patients With Human Epidermal Growth Factor Receptor 2-Positive Breast Cancer and Brain Metastases. J Clin Oncol, 2016. 34(9): p. 945-52.

53. Cortes, J., et al., Afatinib alone or afatinib plus vinorelbine versus investigator's choice of treatment for HER2-positive breast cancer with progressive brain metastases after trastuzumab, lapatinib, or both (LUX-Breast 3): a randomised, open-label, multicentre, phase 2 trial. Lancet Oncol, 2015. 16(16): p. 1700-10.

54. Guirguis, L.M., et al., Safety and efficacy of high-dose interleukin-2 therapy in patients with brain metastases. J Immunother, 2002. 25(1): p. 82-7.

55. Margolin, K., et al., Ipilimumab in patients with melanoma and brain metastases: an open-label, phase 2 trial. Lancet Oncol, 2012. 13(5): p. 459-65.

56. Patel, K.R., et al., Ipilimumab and Stereotactic Radiosurgery Versus Stereotactic Radiosurgery Alone for Newly Diagnosed Melanoma Brain Metastases. Am J Clin Oncol, 2017. 40(5): p. 444-450.

57. Tan, A.C., et al., Immune Checkpoint Inhibitors for Brain Metastases. Curr Oncol Rep, 2017. 19(6): p. 38. 
58. Berghoff, A.S., et al., Immune Checkpoint Inhibitors in Brain Metastases: From Biology to Treatment. Am Soc Clin Oncol Educ Book, 2016. 35: p. e116-22.

59. Harter, P.N., et al., Distribution and prognostic relevance of tumor-infiltrating lymphocytes (TILs) and PD-1/PD-L1 immune checkpoints in human brain metastases. Oncotarget, 2015. 6(38): p. 40836-49.

60. Duchnowska, R., et al., Immune response in breast cancer brain metastases and their microenvironment: the role of the PD-1/PD-L axis. Breast Cancer Res, 2016. 18(1): p. 43.

61. Katz, H. and M. Alsharedi, Immunotherapy in triple-negative breast cancer. Med Oncol, 2017. 35(1): p. 13.

62. Kok, M., et al., LBA14Adaptive phase II randomized non-comparative trial of nivolumab after induction treatment in triple negative breast cancer: TONICtrial. Annals of Oncology, 2017.28(suppl_5): p. mdx440.006-mdx440.006.

63. Nanda, R., et al., Pembrolizumab in Patients With Advanced Triple-Negative Breast Cancer: Phase Ib KEYNOTE-012 Study. J Clin Oncol, 2016. 34(21): p. 2460-7.

64. Atezolizumab Extends Survival for Breast Cancer. Cancer Discov, 2017. 7(6): p. OF10.

65. Chin, K., V.K. Chand, and D.S.A. Nuyten, Avelumab: clinical trial innovation and collaboration to advance anti-PD-L1 immunotherapy. Ann Oncol, 2017. 28(7): p. 1658-1666.

66. Solinas, C., et al., Targeting immune checkpoints in breast cancer: an update of early results. ESMO Open, 2017. 2(5): p. e000255. 
67. Krop, I.E., et al., Trastuzumab emtansine (T-DM1) versus lapatinib plus capecitabine in patients with HER2-positive metastatic breast cancer and central nervous system metastases: a retrospective, exploratory analysis in EMILIA. Ann Oncol, 2015. 26(1): p. 113-9.

68. Bartsch, R., et al., Activity of T-DM1 in Her2-positive breast cancer brain metastases. Clin Exp Metastasis, 2015. 32(7): p. 729-37.

69. Keith, K.C., et al., Activity of Trastuzumab-Emtansine (Tdm1) in Her2-Positive Breast Cancer Brain Metastases: A Case Series. Cancer Treat Commun, 2016. 7: p. 43-46.

70. Jacot, W., et al., Efficacy and safety of trastuzumab emtansine (T-DM1) in patients with HER2-positive breast cancer with brain metastases. Breast Cancer Res Treat, 2016. 157(2): p. 307-318.

71. Okines, A., et al., Development and responses of brain metastases during treatment with trastuzumab emtansine (T-DM1) for HER2 positive advanced breast cancer: A single institution experience. Breast J, 2017.

72. Siegel, R.L., K.D. Miller, and A. Jemal, Cancer Statistics, 2017. CA Cancer J Clin, 2017. 67(1): p. 7-30.

73. Dela Cruz, C.S., L.T. Tanoue, and R.A. Matthay, Lung cancer: epidemiology, etiology, and prevention. Clin Chest Med, 2011. 32(4): p. 605-44.

74. Donington, J.S. and Y.L. Colson, Sex and gender differences in non-small cell lung cancer. Semin Thorac Cardiovasc Surg, 2011. 23(2): p. 137-45.

75. Kiyohara, C. and Y. Ohno, Sex differences in lung cancer susceptibility: a review. Gend Med, 2010. 7(5): p. 381-401. 
76. Fenske, D.C., et al., Systematic Review of Brain Metastases in Patients With NonSmall-Cell Lung Cancer in the United States, European Union, and Japan. Clin Lung Cancer, 2017. 18(6): p. 607-614.

77. Zappa, C. and S.A. Mousa, Non-small cell lung cancer: current treatment and future advances. Transl Lung Cancer Res, 2016. 5(3): p. 288-300.

78. Gazdar, A.F., P.A. Bunn, and J.D. Minna, Small-cell lung cancer: what we know, what we need to know and the path forward. Nat Rev Cancer, 2017. 17(12): p. 725-737.

79. Sholl, L., Molecular diagnostics of lung cancer in the clinic. Transl Lung Cancer Res, 2017. 6(5): p. 560-569.

80. Westcott, P.M. and M.D. To, The genetics and biology of KRAS in lung cancer. Chin J Cancer, 2013. 32(2): p. 63-70.

81. Matikas, A., et al., Targeting KRAS mutated non-small cell lung cancer: A history of failures and a future of hope for a diverse entity. Crit Rev Oncol Hematol, 2017. 110: p. 1-12.

82. Mao, C., et al., KRAS mutations and resistance to EGFR-TKIs treatment in patients with non-small cell lung cancer: a meta-analysis of 22 studies. Lung Cancer, 2010. 69(3): p. 272-8.

83. Siegelin, M.D. and A.C. Borczuk, Epidermal growth factor receptor mutations in lung adenocarcinoma. Lab Invest, 2014. 94(2): p. 129-37.

84. Paez, J.G., et al., EGFR mutations in lung cancer: correlation with clinical response to gefitinib therapy. Science, 2004. 304(5676): p. 1497-500. 
85. Chan, B.A. and B.G. Hughes, Targeted therapy for non-small cell lung cancer: current standards and the promise of the future. Transl Lung Cancer Res, 2015. 4(1): p. 36-54.

86. Bethune, G., et al., Epidermal growth factor receptor (EGFR) in lung cancer: an overview and update. J Thorac Dis, 2010. 2(1): p. 48-51.

87. Bhatt, V.R., et al., Brain metastasis in patients with non-small-cell lung cancer and epidermal growth factor receptor mutations. J Clin Oncol, 2013. 31(25): p. 3162-4.

88. Shackelford, R.E., et al., ALK-rearrangements and testing methods in non-small cell lung cancer: a review. Genes Cancer, 2014. 5(1-2): p. 1-14.

89. Liao, B.C., et al., Treating patients with ALK-positive non-small cell lung cancer: latest evidence and management strategy. Ther Adv Med Oncol, 2015. 7(5): p. 274-90.

90. Lin, J.J. and A.T. Shaw, Differential Sensitivity to Crizotinib: Does EML4-ALK Fusion Variant Matter? J Clin Oncol, 2016. 34(28): p. 3363-5.

91. Woo, C.G., et al., Differential protein stability and clinical responses of EML4ALK fusion variants to various ALK inhibitors in advanced ALK-rearranged nonsmall cell lung cancer. Ann Oncol, 2017. 28(4): p. 791-797.

92. Kayaniyil, S., et al., Treatment patterns and survival in patients with ALK-positive non-small-cell lung cancer: a Canadian retrospective study. Curr Oncol, 2016. 23(6): p. e589-e597.

93. Bergethon, K., et al., ROS1 rearrangements define a unique molecular class of lung cancers. J Clin Oncol, 2012. 30(8): p. 863-70. 
94. Bubendorf, L., et al., Testing for ROS1 in non-small cell lung cancer: a review with recommendations. Virchows Arch, 2016. 469(5): p. 489-503.

95. Shaw, A.T., et al., Crizotinib in ROS1-rearranged non-small-cell lung cancer. N Engl J Med, 2014. 371(21): p. 1963-71.

96. Dagogo-Jack, I. and A.T. Shaw, Expanding the Roster of ROS1 Inhibitors. J Clin Oncol, 2017. 35(23): p. 2595-2597.

97. Omori, S., et al., Changes in PD-L1 expression in non-small cell lung cancer by immunohistochemical analysis. Journal of Clinical Oncology, 2015.

33(15_suppl): p. e22118-e22118.

98. Haratake, N., et al., Positive Conversion of PD-L1 Expression After Treatments with Chemotherapy and Nivolumab. Anticancer Res, 2017. 37(10): p. 5713-5717.

99. He, Y., et al., PD-1, PD-L1 Protein Expression in Non-Small Cell Lung Cancer and Their Relationship with Tumor-Infiltrating Lymphocytes. Med Sci Monit, 2017. 23: p. 1208-1216.

100. Alsaab, H.O., et al., PD-1 and PD-L1 Checkpoint Signaling Inhibition for Cancer Immunotherapy: Mechanism, Combinations, and Clinical Outcome. Front Pharmacol, 2017. 8: p. 561.

101. Seetharamu, N., I.R. Preeshagul, and K.M. Sullivan, New PD-L1 inhibitors in non-small cell lung cancer - impact of atezolizumab. Lung Cancer (Auckl), 2017. 8: p. $67-78$.

102. Dagogo-Jack, I. and A.T. Shaw, Crizotinib resistance: implications for therapeutic strategies. Ann Oncol, 2016. 27 Suppl 3: p. iii42-iii50. 
103. Bui, N., et al., Novel Treatment Strategies for Brain Metastases in Non-small-cell Lung Cancer. Curr Treat Options Oncol, 2016. 17(5): p. 25.

104. Yousefi, M., et al., Lung cancer-associated brain metastasis: Molecular mechanisms and therapeutic options. Cell Oncol (Dordr), 2017. 40(5): p. 419441.

105. Chi, A. and R. Komaki, Treatment of brain metastasis from lung cancer. Cancers (Basel), 2010. 2(4): p. 2100-37.

106. Lekic, M., et al., Outcome of small cell lung cancer (SCLC) patients with brain metastases in a routine clinical setting. Radiol Oncol, 2012. 46(1): p. 54-9.

107. Tsakonas, G., L. De Petris, and S. Ekman, Management of brain metastasized non-small cell lung cancer (NSCLC) - From local treatment to new systemic therapies. Cancer Treat Rev, 2017. 54: p. 122-131.

108. Owen, S. and L. Souhami, The management of brain metastases in non-small cell lung cancer. Front Oncol, 2014. 4: p. 248.

109. Hsu, F., et al., EGFR mutation status on brain metastases from non-small cell lung cancer. Lung Cancer, 2016. 96: p. 101-7.

110. Sekine, A., et al., Metastatic brain tumors from non-small cell lung cancer with EGFR mutations: distinguishing influence of exon 19 deletion on radiographic features. Lung Cancer, 2012. 77(1): p. 64-9.

111. Wardak, Z. and H. Choy, Improving Treatment Options for Brain Metastases From ALK-Positive Non-Small-Cell Lung Cancer. J Clin Oncol, 2016. 34(34): p. 4064-4065. 
112. Ali, A., et al., Survival of patients with non-small-cell lung cancer after a diagnosis of brain metastases. Curr Oncol, 2013. 20(4): p. e300-6.

113. Walbert, T. and M.R. Gilbert, The role of chemotherapy in the treatment of patients with brain metastases from solid tumors. Int J Clin Oncol, 2009. 14(4): p. 299-306.

114. Elaimy, A.L., et al., Long-term survival in a patient with multiple brain metastases from small-cell lung cancer treated with gamma knife radiosurgery on four occasions: a case report. Case Rep Neurol Med, 2012. 2012: p. 276189.

115. Niemiec, M., et al., Characteristics of long-term survivors of brain metastases from lung cancer. Rep Pract Oncol Radiother, 2011. 16(2): p. 49-53.

116. Mulvenna, P., et al., Dexamethasone and supportive care with or without whole brain radiotherapy in treating patients with non-small cell lung cancer with brain metastases unsuitable for resection or stereotactic radiotherapy (QUARTZ): results from a phase 3, non-inferiority, randomised trial. Lancet, 2016. 388(10055): p. 2004-2014.

117. Won, Y.K., et al., Stereotactic radiosurgery for brain metastasis in non-small cell lung cancer. Radiat Oncol J, 2015. 33(3): p. 207-16.

118. Schuette, W., Treatment of brain metastases from lung cancer: chemotherapy. Lung Cancer, 2004. 45 Suppl 2: p. S253-7.

119. Lin, J., et al., Current and emerging treatments for brain metastases. Oncology (Williston Park), 2015. 29(4): p. 250-7.

120. Reveiz, L., J.R. Rueda, and A.F. Cardona, Chemotherapy for brain metastases from small cell lung cancer. Cochrane Database Syst Rev, 2012(6): p. CD007464. 
121. Abdel Karim, N., et al., Systemic Chemotherapy for Progression of Brain Metastases in Extensive-Stage Small Cell Lung Cancer. Case Rep Oncol Med, 2015. 2015: p. 620582.

122. Barlesi, F., et al., Pemetrexed and cisplatin as first-line chemotherapy for advanced non-small-cell lung cancer (NSCLC) with asymptomatic inoperable brain metastases: a multicenter phase II trial (GFPC 07-01). Ann Oncol, 2011. 22(11): p. 2466-70.

123. Dinglin, X.X., et al., Pemetrexed and cisplatin combination with concurrent whole brain radiotherapy in patients with brain metastases of lung adenocarcinoma: a single-arm phase II clinical trial. J Neurooncol, 2013. 112(3): p. 461-6.

124. He, Q., et al., Phase II Study of the Efficacy and Safety of High-dose Pemetrexed in Combination with Cisplatin Versus Temozolomide for the Treatment of Nonsmall Cell Lung Cancer with Brain Metastases. Anticancer Res, 2017. 37(8): p. 4711-4716.

125. McGranahan, T. and S. Nagpal, A Neuro-oncologist's Perspective on Management of Brain Metastases in Patients with EGFR Mutant Non-small Cell Lung Cancer. Curr Treat Options Oncol, 2017. 18(4): p. 22.

126. Socinski, M.A., et al., Safety of bevacizumab in patients with non-small-cell lung cancer and brain metastases. J Clin Oncol, 2009. 27(31): p. 5255-61.

127. Bennouna, J., et al., Bevacizumab in Combination with Platinum-Based Chemotherapy in Patients with Advanced Non-Squamous Non-Small Cell Lung 
Cancer with or without Brain Metastases: A French Cohort Study (EOLE). Oncology, 2017.

128. Guinde, J., et al., Bevacizumab Plus Radiosurgery for Nonsquamous Non-Small Cell Lung Cancer Patients with Brain Metastases: Safe Combination? World Neurosurg, 2017. 107: p. 1047 e1-1047 e4.

129. Stefanou, D., et al., Bevacizumab, pemetrexed and carboplatin in first-line treatment of non-small cell lung cancer patients: Focus on patients with brain metastases. Oncol Lett, 2016. 12(6): p. 4635-4642.

130. Khasraw, M., et al., Intracranial hemorrhage in patients with cancer treated with bevacizumab: the Memorial Sloan-Kettering experience. Ann Oncol, 2012. 23(2): p. $458-63$.

131. Besse, B., et al., Bevacizumab in Patients with Nonsquamous Non-Small Cell Lung Cancer and Asymptomatic, Untreated Brain Metastases (BRAIN): A Nonrandomized, Phase II Study. Clin Cancer Res, 2015. 21(8): p. 1896-903.

132. Danciu, O.C., et al., Prolonged activity of bevacizumab in adenocarcinoma of the lung with multiple brain metastases. Med Oncol, 2012. 29(4): p. 2619-22.

133. De Braganca, K.C., et al., Efficacy and safety of bevacizumab in active brain metastases from non-small cell lung cancer. J Neurooncol, 2010. 100(3): p. 4437.

134. Besse, B., et al., Bevacizumab safety in patients with central nervous system metastases. Clin Cancer Res, 2010. 16(1): p. 269-78. 
135. Dziadziuszko, R., et al., Temozolomide in patients with advanced non-small cell lung cancer with and without brain metastases. a phase II study of the EORTC Lung Cancer Group (08965). Eur J Cancer, 2003. 39(9): p. 1271-6.

136. Deng, X., et al., The efficacy and roles of combining temozolomide with whole brain radiotherapy in protection neurocognitive function and improvement quality of life of non-small-cell lung cancer patients with brain metastases. BMC Cancer, 2017. 17(1): p. 42.

137. Giorgio, C.G., et al., Oral temozolomide in heavily pre-treated brain metastases from non-small cell lung cancer: phase II study. Lung Cancer, 2005. 50(2): p. 247-54.

138. Ballard, P., et al., Preclinical Comparison of Osimertinib with Other EGFR-TKIs in EGFR-Mutant NSCLC Brain Metastases Models, and Early Evidence of Clinical Brain Metastases Activity. Clin Cancer Res, 2016. 22(20): p. 5130-5140.

139. Zeng, Y.D., et al., Blood-brain barrier permeability of gefitinib in patients with brain metastases from non-small-cell lung cancer before and during whole brain radiation therapy. Oncotarget, 2015. 6(10): p. 8366-76.

140. Ceresoli, G.L., et al., Gefitinib in patients with brain metastases from non-smallcell lung cancer: a prospective trial. Ann Oncol, 2004. 15(7): p. 1042-7.

141. Hotta, K., et al., Effect of gefitinib ('Iressa', ZD1839) on brain metastases in patients with advanced non-small-cell lung cancer. Lung Cancer, 2004. 46(2): p. 255-61. 
142. Chiu, C.H., et al., Gefitinib is active in patients with brain metastases from nonsmall cell lung cancer and response is related to skin toxicity. Lung Cancer, 2005. 47(1): p. 129-38.

143. Leitner, C., et al., Progressive brain metastases in an EGFR mutated adenocarcinoma of the lung: Response to gefitinib after progression on erlotinib. Cancer Treatment Communications, 2014. 2(1): p. 4-7.

144. Deng, Y., et al., The concentration of erlotinib in the cerebrospinal fluid of patients with brain metastasis from non-small-cell lung cancer. Mol Clin Oncol, 2014. 2(1): p. 116-120.

145. Weber, B., et al., Erlotinib accumulation in brain metastases from non-small cell lung cancer: visualization by positron emission tomography in a patient harboring a mutation in the epidermal growth factor receptor. J Thorac Oncol, 2011. 6(7): p. 1287-9.

146. Porta, R., et al., Brain metastases from lung cancer responding to erlotinib: the importance of EGFR mutation. Eur Respir J, 2011. 37(3): p. 624-31.

147. Nonagase, Y., et al., Afatinib-refractory brain metastases from EGFR-mutant non-small-cell lung cancer successfully controlled with erlotinib: a case report. Anticancer Drugs, 2016. 27(3): p. 251-3.

148. Nakahama K, T.A., Taniguchi Y, Naoki Y, Kanazu M, et al., Comparing Gefitinib and Erlotinib With Regard To Brain Metastases Recurrence in EGFRMutant Non-Small Cell Lung Cancer Patients. J Clin Exp Oncol 2017.

149. Brower, J.V. and H.I. Robins, Erlotinib for the treatment of brain metastases in non-small cell lung cancer. Expert Opin Pharmacother, 2016. 17(7): p. 1013-21. 
150. Bai, H. and B. Han, The effectiveness of erlotinib against brain metastases in nonsmall cell lung cancer patients. Am J Clin Oncol, 2013. 36(2): p. 110-5.

151. Lee, S.M., et al., Randomized trial of erlotinib plus whole-brain radiotherapy for NSCLC patients with multiple brain metastases. J Natl Cancer Inst, 2014. 106(7).

152. Welsh, J.W., et al., Phase II trial of erlotinib plus concurrent whole-brain radiation therapy for patients with brain metastases from non-small-cell lung cancer. J Clin Oncol, 2013. 31(7): p. 895-902.

153. Ulahannan, D. and S.M. Lee, Erlotinib plus concurrent whole-brain radiation therapy for non-small cell lung cancers patients with multiple brain metastases. Transl Lung Cancer Res, 2016. 5(2): p. 208-11.

154. Schuler, M., et al., First-Line Afatinib versus Chemotherapy in Patients with NonSmall Cell Lung Cancer and Common Epidermal Growth Factor Receptor Gene Mutations and Brain Metastases. J Thorac Oncol, 2016. 11(3): p. 380-90.

155. van Hoppe, S., et al., Breast cancer resistance protein (BCRP/ABCG2) and Pglycoprotein (P-gp/ABCB1) transport afatinib and restrict its oral availability and brain accumulation. Pharmacol Res, 2017. 120: p. 43-50.

156. Zhang, S.R., et al., Efficacy of afatinib, an irreversible ErbB family blocker, in the treatment of intracerebral metastases of non-small cell lung cancer in mice. Acta Pharmacol Sin, 2017. 38(2): p. 233-240.

157. Rangachari, D., et al., Brain metastases in patients with EGFR-mutated or ALKrearranged non-small-cell lung cancers. Lung Cancer, 2015. 88(1): p. 108-11.

158. Bollinger, M.K., A.S. Agnew, and G.P. Mascara, Osimertinib: A third-generation tyrosine kinase inhibitor for treatment of epidermal growth factor receptor- 
mutated non-small cell lung cancer with the acquired Thr790Met mutation. $\mathrm{J}$ Oncol Pharm Pract, 2017: p. 1078155217712401.

159. Hata, A., et al., Rebiopsy of non-small cell lung cancer patients with acquired resistance to epidermal growth factor receptor-tyrosine kinase inhibitor: Comparison between T790M mutation-positive and mutation-negative populations. Cancer, 2013. 119(24): p. 4325-32.

160. Goss, G., et al., MA16.11 CNS Response to Osimertinib in Patients with T790MPositive Advanced NSCLC: Pooled Data from Two Phase II Trials. Journal of Thoracic Oncology. 12(1): p. S440-S441.

161. Mok, T.S., et al., Osimertinib or Platinum-Pemetrexed in EGFR T790M-Positive Lung Cancer. N Engl J Med, 2017. 376(7): p. 629-640.

162. Remon, J., et al., The APPLE Trial: Feasibility and Activity of AZD9291 (Osimertinib) Treatment on Positive PLasma T790M in EGFR-mutant NSCLC Patients. EORTC 1613. Clin Lung Cancer, 2017. 18(5): p. 583-588.

163. Tan, C.S., B.C. Cho, and R.A. Soo, Treatment options for EGFR mutant NSCLC with CNS involvement-Can patients BLOOM with the use of next generation EGFR TKIs? Lung Cancer, 2017. 108: p. 29-37.

164. Zhang, I., et al., Targeting brain metastases in ALK-rearranged non-small-cell lung cancer. Lancet Oncol, 2015. 16(13): p. e510-21.

165. Costa, D.B., et al., CSF concentration of the anaplastic lymphoma kinase inhibitor crizotinib. J Clin Oncol, 2011. 29(15): p. e443-5. 
166. Yoshida, T., et al., Clinical impact of crizotinib on central nervous system progression in ALK-positive non-small lung cancer. Lung Cancer, 2016. 97: p. 43-7.

167. Costa, D.B., et al., Clinical Experience With Crizotinib in Patients With Advanced ALK-Rearranged Non-Small-Cell Lung Cancer and Brain Metastases. J Clin Oncol, 2015. 33(17): p. 1881-8.

168. Tang, S.C., et al., Increased oral availability and brain accumulation of the ALK inhibitor crizotinib by coadministration of the P-glycoprotein $(A B C B 1)$ and breast cancer resistance protein (ABCG2) inhibitor elacridar. Int J Cancer, 2014. 134(6): p. 1484-94.

169. Kort, A., et al., Brain accumulation of the EML4-ALK inhibitor ceritinib is restricted by $P$-glycoprotein $(P-G P / A B C B 1)$ and breast cancer resistance protein (BCRP/ABCG2). Pharmacol Res, 2015. 102: p. 200-7.

170. Kim, D.W., et al., Activity and safety of ceritinib in patients with ALK-rearranged non-small-cell lung cancer (ASCEND-1): updated results from the multicentre, open-label, phase 1 trial. Lancet Oncol, 2016. 17(4): p. 452-63.

171. Crino, L., et al., Multicenter Phase II Study of Whole-Body and Intracranial Activity With Ceritinib in Patients With ALK-Rearranged Non-Small-Cell Lung Cancer Previously Treated With Chemotherapy and Crizotinib: Results From ASCEND-2. J Clin Oncol, 2016. 34(24): p. 2866-73.

172. Felip, E., et al., 141PD: Whole body and intracranial efficacy of ceritinib in patients (pts) with crizotinib (CRZ) pretreated, ALK-rearranged (ALK+) nonsmall cell lung cancer (NSCLC) and baseline brain metastases (BM): Results 
from ASCEND-1 and ASCEND-2 trials. J Thorac Oncol, 2016. 11(4 Suppl): p. S118-9.

173. Shaw, A.T., et al., Ceritinib versus chemotherapy in patients with ALKrearranged non-small-cell lung cancer previously given chemotherapy and crizotinib (ASCEND-5): a randomised, controlled, open-label, phase 3 trial. Lancet Oncol, 2017. 18(7): p. 874-886.

174. Kodama, T., et al., Antitumor activity of the selective ALK inhibitor alectinib in models of intracranial metastases. Cancer Chemother Pharmacol, 2014. 74(5): $\mathrm{p}$. 1023-8.

175. Ou, S.H., et al., Alectinib in Crizotinib-Refractory ALK-Rearranged Non-SmallCell Lung Cancer: A Phase II Global Study. J Clin Oncol, 2016. 34(7): p. 661-8.

176. Gadgeel S, P.S., Mok T, et al., Alectinib vs crizotinib in treatment-naüve ALK+ NSCLC: CNS efficacy results from the ALEX study. . ESMO Congress, 2017.

177. Novello S, M.J., Oh I, et al., Primary results from the phase III ALUR study of alectinib versus chemotherapy in previously treated $A L K+$ non-small-cell lung cancer (NSCLC). ESMO Congress, 2017.

178. Gettinger, S., et al., OA08.06 Brigatinib Activity in Patients with ALK+ NSCLC and Intracranial CNS Metastases in Two Clinical Trials. Journal of Thoracic Oncology. 12(1): p. S273-S274.

179. Kim, D.W., et al., Brigatinib in Patients With Crizotinib-Refractory Anaplastic Lymphoma Kinase-Positive Non-Small-Cell Lung Cancer: A Randomized, Multicenter Phase II Trial. J Clin Oncol, 2017. 35(22): p. 2490-2498. 
180. Collier, T.L., et al., Brain Penetration of the ROSI/ALK Inhibitor Lorlatinib Confirmed by PET. Mol Imaging, 2017. 16: p. 1536012117736669.

181. Felip, E., et al., MA07.11 Safety and Efficacy of Lorlatinib (PF-06463922) in Patients with Advanced ALK+ or ROS1+Non-Small-Cell Lung Cancer (NSCLC). Journal of Thoracic Oncology. 12(1): p. S383-S384.

182. Farago, A.F., et al., Durable Clinical Response to Entrectinib in NTRK1Rearranged Non-Small Cell Lung Cancer. J Thorac Oncol, 2015. 10(12): p. 16704.

183. Takada, K., et al., The Significance of the PD-L1 Expression in Non-Small-Cell Lung Cancer: Trenchant Double Swords as Predictive and Prognostic Markers. Clin Lung Cancer, 2017.

184. Zhang, M., et al., PD-L1 expression in lung cancer and its correlation with driver mutations: a meta-analysis. Sci Rep, 2017. 7(1): p. 10255.

185. Ramos-Esquivel, A., et al., Anti-PD-1/anti-PD-L1 immunotherapy versus docetaxel for previously treated advanced non-small cell lung cancer: a systematic review and meta-analysis of randomised clinical trials. ESMO Open, 2017. 2(3): p. e000236.

186. Lu, J. and R.A. Ramirez, The Role of Checkpoint Inhibition in Non-Small Cell Lung Cancer. Ochsner J, 2017. 17(4): p. 379-387.

187. Assi, H.I., et al., Immune checkpoint inhibitors in advanced non-small cell lung cancer. Cancer, 2017. 
188. Aguiar, P.N., Jr., et al., Immune checkpoint inhibitors for advanced non-small cell lung cancer: emerging sequencing for new treatment targets. ESMO Open, 2017. 2(3): p. e000200.

189. Ahn, M.J., et al., EGFR TKI combination with immunotherapy in non-small cell lung cancer. Expert Opin Drug Saf, 2017. 16(4): p. 465-469.

190. Karachaliou, N., et al., The combination of checkpoint immunotherapy and targeted therapy in cancer. Ann Transl Med, 2017. 5(19): p. 388.

191. Liu, B., Y. Song, and D. Liu, Recent development in clinical applications of PD-1 and PD-L1 antibodies for cancer immunotherapy. J Hematol Oncol, 2017. 10(1): p. 174.

192. Gettinger, S. and K. Politi, PD-1 Axis Inhibitors in EGFR-and ALK-Driven Lung Cancer: Lost Cause? Clin Cancer Res, 2016. 22(18): p. 4539-41.

193. Ota, K., et al., Induction of PD-L1 Expression by the EML4-ALK Oncoprotein and Downstream Signaling Pathways in Non-Small Cell Lung Cancer. Clin Cancer Res, 2015. 21(17): p. 4014-21.

194. Reck, M., et al., Pembrolizumab versus Chemotherapy for PD-L1-Positive NonSmall-Cell Lung Cancer. N Engl J Med, 2016. 375(19): p. 1823-1833.

195. Goldberg, S.B., et al., Pembrolizumab for patients with melanoma or non-smallcell lung cancer and untreated brain metastases: early analysis of a nonrandomised, open-label, phase 2 trial. Lancet Oncol, 2016. 17(7): p. 976-983.

196. Watanabe, H., et al., The effect of nivolumab treatment for central nervous system metastases in non-small cell lung cancer. Journal of Clinical Oncology, 2017. 35(15_suppl): p. e20601-e20601. 
197. Dudnik, E., et al., Intracranial response to nivolumab in NSCLC patients with untreated or progressing CNS metastases. Lung Cancer, 2016. 98: p. 114-117.

198. Antonia, S.J., et al., Durvalumab after Chemoradiotherapy in Stage III NonSmall-Cell Lung Cancer. N Engl J Med, 2017. 377(20): p. 1919-1929.

199. Lukas, R.V., Gandhi M., O'hear C., et al., Safety and efficacy analyses of atezolizumab in advanced non-small cell lung cancer (NSCLC) patients with or without baseline brain metastases. ESMO Congress, 2017.

200. Gulley, J.L., et al., Avelumab for patients with previously treated metastatic or recurrent non-small-cell lung cancer (JAVELIN Solid Tumor): dose-expansion cohort of a multicentre, open-label, phase $1 b$ trial. Lancet Oncol, 2017. 18(5): p. 599-610.

201. Zhu, J., et al., Immunotherapy (excluding checkpoint inhibitors) for stage I to III non-small cell lung cancer treated with surgery or radiotherapy with curative intent. Cochrane Database Syst Rev, 2017. 12: p. CD011300.

202. Davis, M.E., Glioblastoma: Overview of Disease and Treatment. Clin J Oncol Nurs, 2016. 20(5): p. S2-8.

203. Omuro, A. and L.M. DeAngelis, Glioblastoma and other malignant gliomas: a clinical review. JAMA, 2013. 310(17): p. 1842-50.

204. Noroxe, D.S., H.S. Poulsen, and U. Lassen, Hallmarks of glioblastoma: a systematic review. ESMO Open, 2016. 1(6): p. e000144.

205. Bobola, M.S., et al., Minimally cytotoxic doses of temozolomide produce radiosensitization in human glioblastoma cells regardless of MGMT expression. Mol Cancer Ther, 2010. 9(5): p. 1208-18. 
206. Danaila, L., G. Ghyka, and C. Ursaciuc, Interleukin-2 (IL-2) in the treatment of malignant brain tumors (glioblastomas). Rom J Neurol Psychiatry, 1993. 31(3-4): p. 195-206.

207. Weller, M., et al., Rindopepimut with temozolomide for patients with newly diagnosed, EGFRvIII-expressing glioblastoma (ACT IV): a randomised, doubleblind, international phase 3 trial. Lancet Oncol, 2017. 18(10): p. 1373-1385. 


\section{CHAPTER 3}

\section{PHARMACOKINETICS OF LUCIFERIN IN PRECLINICAL BRAIN METASTASES AND PRIMARY BRAIN TUMOR MODELS}

\subsection{Introduction}

Brain tumors are associated with significant mortality and morbidity, with patients succumbing to the tumors within two years [1]. Approximately 10-20\% of intracranial lesions arise from cells of the central nervous system (CNS), such as glial cells which when mutated can result in the formation of glioblastoma [2, 3]. Of significance, and often overlooked in the preclinical literature is $80 \%$ of brain lesions start as a cancer in a distant tissue outside the CNS. The three dominant metastatic originating cancers are lung, breast and melanoma [4]. There have been a significant number of preclinical primary CNS and metastatic CNS models that have been recently developed. Accordingly, there is a substantial need to be able to longitudinally quantify tumor growth and response within the skull [5].

Quantifying preclinical intracranial longitudinal tumor response to therapy can be difficult. Two common current modalities include magnetic resonance imaging (MRI) and computed-axial tomography (CT) [6]. While these imaging modalities are noninvasive, they are often time-consuming and expensive. Direct cell-labeling with quantum dots or use of radionucleotides are similarly expensive and may cause significant toxicity $[7,8]$. These imaging methods are being supplanted by the use of luciferase. Three types of luciferase exist: Gaussia, Renilla, and Firefly, the latter of which is used most commonly. Incorporation of the firefly luciferase (Fluc) enzyme into 
cells allows for non-invasive, non-toxic visualization and quantification in both in-vitro and in-vivo settings [9].

In bioluminescence, Fluc oxidizes its substrate D-luciferin in an ATP-dependent reaction to give off photons of light. These photons have an emission maximum ( $\lambda$ max $)$ of approximately $560 \mathrm{~nm}$, which exists close to far-red spectra and are easily distinguished against background light scatter for quantification accuracy [10]. As vertebral systems do not endogenously express Fluc and since ATP is required in the oxidation mechanism to produce photons, only living cells incorporated with Fluc will produce signal, with the amount of signal proportional to amount of photon-producing cells [10-12]. Neither Fluc incorporation nor D-luciferin create toxic effects in vertebrates or cells $[13,14]$. This allows Fluc and D-luciferin to be a highly sensitive and reliable pairing for both real-time and longitudinal cellular proliferation, cellular migration, and tumor burden $[10,15]$. This process is bioluminescence imaging (BLI).

The rate of photon production from D-luciferin metabolism generally exhibit a gradual increase and decrease surrounding peak intensity [16]. It is suggested that this BLI peak that most accurately represents the extent of tumor burden [17]. Therefore, performing a D-luciferin pharmacokinetic profile maximizes measurement of tumor growth, chemotherapy response, and reproducibility in longitudinal studies. Each cell line, method of luciferin administration, and mode of tumor inoculation will produce different pharmacokinetic profiles [15, 18].

Herein, we utilize intraperitoneal injections of D-luciferin and firefly luciferase pharmacokinetics to characterize BLI peak and minimal-error imaging times in three preclinical metastatic brain and one intracranial cancer models. These models include the 
human HER2+ JIMT-1 and the triple negative MDA-MB-231 breast cancer lines, the EGFR-mutant PC-9 lung cancer line, and the intracranial U251 glioblastoma line. We observed an inverse relationship between peak photon production and length of optimal imaging time: while peak BLI signal is comparatively delayed in the breast-brain metastatic models, the optimal imaging duration is significantly longer than lung-brain or intracranial tumors $(\mathrm{p}<0.05)$. Similarly, an inverse relationship between time to peakBLI and statistically error-free period was observed: the lung-brain metastatic and intracranial model had double the imaging window than the breast-brain metastatic models $(\mathrm{p}<0.05)$. Large variability exists within the first 10 minutes of imaging in all models, and in intracranially injected models, this leaves sparse time for optimal quantification. Significant differences in area under the curve for total photon emission exists within groups of the in-vivo and in-vitro models $(\mathrm{p}<0.05)$. In-vitro luciferin assays using the same cell lines produce consistent signal production at similar values to their invivo peaks and greatly vary in their photon production and plateau region of optimal signal capture, which suggest that each cell line has different intrinsic luciferase kinetics.

\subsection{Methods and Materials}

\section{Chemicals and reagents}

A lentivirus containing Fluc with the hygromycin selection marker was purchased from Biosettia (GlowCell-16h). $10 \mu \mathrm{L}$ of viral vector and $10 \mu \mathrm{L}$ of polybrene (Santa Cruz, sc-134220) was added to each well in a 6-well plate of JIMT-1-Br or U251 at 70\% confluence. Plates were spun at 1000 RPM for 60 minutes and placed in a 37C incubator overnight. The following morning, cells were introduced to hygromycin at a concentration of $10 \mu \mathrm{L} / \mathrm{mL}$ in media to ensure successful transduction. The PC-9-Br line 
was transduced with firefly luciferase plasmid from addgene (32904) by the methods in Chapter 5.2 and selected for successful incorporation with G418 at $10 \mu \mathrm{L} / \mathrm{mL}$. The MDA-MB-231 cell line was pre-transduced with Fluc and kindly provided by Dr. Patricia Steeg from the NIH. Cells were visualized in the IVIS Spectrum CT for luciferase expression prior to injection into mice. Firefly D-luciferase potassium salt was purchased from Perkin Elmer (Waltham, MA). All chemicals and reagents were of analytical grade and used as supplied.

\section{Cell Culture}

The JIMT-1-Br and MDA-MB-231-Br brain-seeking breast cancer lines were a kind gift from Dr. Patricia Steeg at the National Cancer Institute. The U251 glioblastoma line was provided by Dr. Gordon Meares at West Virginia University. The PC-9-Br lung cancer line was provided by Dr. Lori Hazlehurst at West Virginia University. JIMT-1-Br, MDA-MB-231-Br, and U251 cells were grown in DMEM with 10\% fetal bovine serum and $5 \%$ penicillin-streptomycin, along with hygromycin at $10 \mu \mathrm{L} / \mathrm{mL}$. PC-9-Br cells were grown in RPMI with 10\% FBS, 5\% penicillin-streptomycin, and G4918 at $10 \mu \mathrm{L} / \mathrm{mL}$. All cells were used in passages $1-15$, utilized at $70-80 \%$ confluence, and maintained at $37^{\circ} \mathrm{C}$ with $5 \% \mathrm{CO} 2$.

\section{Brain Tumor Models}

Female athymic nu/nu mice were purchased from Charles River Laboratories (Wilmington, MA). All animals were approximately 6-8 weeks on time of model initiation. Mice were anesthetized under $2 \%$ isoflurane and injected with 150,000175,000 cancer cells via the cardiac left ventricle using a stereotactic device (Stoelting, 
Wood Dale, IL). The same stereotactic device was used to inject 500,000 cells in the intracranial model. U251 cells were injected slowly through the cranium approximately $1.5 \mathrm{~mm}$ below and $1 \mathrm{~mm}$ to the right of bregma at $1 \mu \mathrm{L} /$ minute for a 5 -minute time period. Animals were monitored until fully recovered. Experiments were conducted under the institutional Animal Care and Use Committee protocol (WVU \#1604001892). All procedures performed in studies involving animals were in accordance with the ethical standards of the institution or practice at which the studies were conducted. This article does not contain any studies with human participants performed by any of the authors.

\section{In-vivo pharmacokinetics}

Bioluminescence was captured and quantified on the IVIS Spectra CT (PerkinElmer). Animals were anesthetized and laid supine on the imaging field, then given IP injections with $150 \mathrm{mg} / \mathrm{kg}$ of D-luciferin. Immediately after injection, image acquisition was initiated and photon emission was captured every 1-5 minutes for a 4560-minute sequence. Signal was captured using auto exposure, medium binning, F/Stop 1, on Stage D. A region of interest was drawn around the cranium at each time point and photon emission was collected. This region of interest is defined as radiance (photons $/ \mathrm{sec} / \mathrm{cm}^{2} / \mathrm{steridian)}$ [19]. After radiance collection each animal's signal was additionally normalized from peak bioluminescence signal for between-groups comparison and plotted over time of capture (Fig. 3.1). Area Under the Curve (AUC) was calculated using radiance and normalized data for imaging duration via GraphPad Prism 6.

\section{In-vitro pharmacokinetics}


To correlate in-vivo bioluminescence with in-vitro assays, pharmacokinetic profiling of these cell lines were performed on clear-bottom black 96-well plates (B-D \#353220). Cancer cells were seeded at a concentration of 5,000 cells per well and incubated overnight. The following day, D-luciferin at $15 \mu \mathrm{g} / 100 \mu \mathrm{L}$ was given to each well and immediately imaged on the IVIS Spectrum CT every 1 minute for a 30-minute sequence, using auto exposure, medium binning, F/Step 1, and on Stage D. Signal at each minute was averaged across 96 wells, then plotted over time as radiance (photons $/ \mathrm{sec} / \mathrm{cm}^{2} / \mathrm{steridian)}$ and then separately normalized for additional comparison.

\section{Exponential decay}

The exponential decay equation $\mathrm{X}_{\mathrm{t}} * \mathrm{e}^{-\mathrm{kt}}$, was modified for bioluminescence: $\mathrm{P}_{\mathrm{t}} * \mathrm{e}^{-}$

kt; where $\mathrm{P}_{\mathrm{t}}$ and $\mathrm{k}$ represents the amount of photons/second at time $t$. In vivo, $\mathrm{k}$ represents absorption $(\alpha)$ or elimination $(\beta)$; in-vitro this represents fast $\left(\mathrm{K}_{\text {fast }}\right)$ or slow $\left(\mathrm{K}_{\text {slow }}\right)$ elimination. This equation was applied to radiance and normalized values to obtain an $\alpha$ absorption slope value for each model.

\section{Statistics}

The D'Agostino and Pearson omnibus normality test was conducted to confirm Gaussian distribution of values prior to calculations. A one-way ANOVA for multiple comparisons using Tukey's or Dunnet's multiple comparisons test was performed to compare radiance and normalized kinetic curves and peaks. Time within bioluminescence values statistically insignificant from peak values indicates a duration of minimal error. Error range was calculated by averaging the range of signal in 10-minute intervals. A one-way ANOVA was performed to compare each model's in-vivo and in-vitro AUC 
values. To calculate $\alpha$ and $\beta$ slopes for in-vivo luciferin kinetics, one-phase kinetic modeling was used. A two-phase kinetic modeling was used for in-vitro kinetics to define

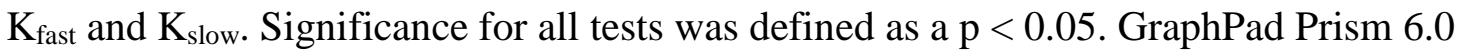
was used to calculate all statistics and produce all graphs.

\subsection{Results}

Peak bioluminescence occurs faster in the intracranial model compared to metastatic models

To determine peak BLI timing, we injected Fluc-transduced tumor-bearing mice with D-luciferin and captured the rate of photons emitted through time (radiance). For distinguishing purposes, data was normalized and all points are plotted at 4-6 minute intervals. Minimal-error imaging is defined as signal not statistically significant $(\mathrm{p}<$ 0.05) from peak value. Radiance data is shown in Fig 3.2. Significant variation exists between and within animal groups, so results will focus on normalized data. In the MDAMB-231-Br line $(n=4)$, peak BLI occurred at $20 \pm 2.7$ minutes, with significant signal difference occurring before 15 minutes and after 25 minutes (Fig. 3.3a). In the JIMT-1Br line ( $\mathrm{n}=3)$, peak BLI occurred at $16 \pm 1.2$ minutes, with a minimal-error duration of 10 to 24 minutes (Fig. 3.3b). In the PC-9-Br line $(\mathrm{n}=4)$, peak BLI occurred at $12 \pm 3.1$ minutes, with minimal-error occurring before 5 minutes and after 24 minutes. (Fig. 3.3c). For the U251 line $(\mathrm{n}=3)$, peak BLI occurred at 9 minutes, with significant signal difference occurring before 5 minutes and after 21 minutes (Fig. 3.3d). The results are summarized in Table 3.1. The amount of signal captured $\left(\mathrm{P}_{\max }\right)$ at peak BLI follow peak BLI timing (Table 3.2). Normalized in-vivo data is represented in Table 3.3. 


\section{Optimal bioluminescence capture timing is longer in the metastatic model}

\section{compared to the intracranial model}

After creating luciferin pharmacokinetic curves for each model, normalized signal ranges from peak was calculated in 10-minute blocks. It was found that the 10-20 minute time block contained the smallest range of signal in most models, displaying an intensity above approximately 95\% of peak BLI (Table 3.4). Similar variability of signal and optimal timing were described in another study of luciferin BLI [20]. Time correlating to $>95 \%$ of peak BLI signal was 15-25 minutes for the MDA-MB-231 model (Fig. 3.3a), 12-20 minutes for the JIMT-1 model (Fig. 3.3b), 9-14 minutes for the PC-9 model (Fig. 3.3c), and 7-12 minutes for the U251 model (Fig. 3.3d); summarized in Table 3.1.

\section{In-vitro bioluminescence reaches a plateau at similar times to in-vivo peak}

\section{values}

To correlate in-vivo models with their in-vitro counterparts, 5,000 cancer cells were added to 96 clear-bottom black-welled plates and incubated overnight. The following day, luciferin was added, followed by quantification and data normalization. Qualitative 5-minute time lapses are shown (Fig. 3.4a). A plateau was defined as signal change less than $1 \%$ between normalized values. In the PC-9 model, BLI signal rapidly dropped off until reaching plateau at $70 \%$ of initial peak value at 14 minutes (Fig. 3.4c). This timing qualitatively corresponds exactly with the in-vivo peak seen in this model. In the U251 model, BLI signal rapidly dropped off until reaching plateau at $43 \%$ of initial peak value at 7 minutes (Fig. 3.4d). This timing qualitatively agrees with the in-vivo peak luminescence in this model seen at 9 minutes. This qualitatively parallels the in-vivo peak seen at 20 minutes in this model. The JIMT-1 line diverges from these findings. While 
the in-vivo peak occurs at 16 minutes, the in-vitro plateau begins at 7 minutes and lasts until 18 minutes, at which point signal begins to fall. Signal within the plateau was $85 \%$ of initial peak value (Fig. 3.4b).

Normalized in-vivo a slopes and radiance $\beta$ slopes are correlated with peak invivo bioluminescence times

With regards to absorption slopes, PC-9 had the fastest slope (0.3689) and JIMT-1 had the slowest slope (0.0919), with U251 (0.1476) and MDA-MB-231 (0.2161) in between (Table 3.2). A linear regression revealed an $\mathrm{r}^{2}$ of 0.01 , indicating no correlation. Normalized $\alpha$ values resemble radiance $\alpha$ values: the PC-9 line had the steepest $\alpha$ slope of 0.4862, while the JIMT-1 line had the lowest $\alpha$ slope of 0.11 . The $\alpha$ of the MDA-MB-231 line of 0.2017 and U251 of 0.2980 fell in between (Table 3.3). A linear regression of peak luminescence times and normalized $\alpha$ slope values produced an $r^{2}$ of 0.32 , indicating modest correlation. Radiance data shows that the U251 model has the highest $B$ value of 0.022 photons/sec, whereas the MDA-MB-231 model has the lowest $\beta$ value of 0.0127 photons/sec (Table 3.2). The radiance $B$ of the PC-9 (0.0128) and JIMT-1 (0.0220) fell in between (Table 3.2). A linear regression of radiance $\beta$ and peak luminescence times had an $\mathrm{r}^{2}$ of 0.33 , indicating modest correlation. In contrast, the normalized $\beta$ luciferin elimination slope was the slowest in the PC-9 line at 0.0036 followed closely by the MDA-MB-231 of 0.0053 , then U251 at 0.0137 , and was the fastest in the JIMT-1 at 0.0192 (Table 3.3). A linear regression of normalized $B$ slope values and peak bioluminescence times produced an $r^{2}$ of 0.02 , indicating no correlation.

\section{Radiance and normalized $A U C_{0-60}$ are correlated with peak bioluminescence}

\section{times}


Radiance was plotted over time and the AUC (total photons given off during imaging time) for length of capture was quantified. The $\mathrm{AUC}_{0-60}$ was the highest in the U251 $\left(1.2 \times 10^{8}\right)$ and lowest in the MDA-MB-231 model $\left(4.4 \times 10^{3}\right)$, with JIMT $(7.4 \mathrm{x}$ $\left.10^{7}\right)$ and PC-9 $\left(3.0 \times 10^{6}\right)$ falling in between (Table 3.2). This agrees with peak in-vivo bioluminescence timing, and a linear regression analyses produced an $r^{2}$ of 0.37 . A oneway ANOVA showed significant differences in radiance AUC between all models $(\mathrm{p}<$ 0.05). In contrast, the normalized $\mathrm{AUC}_{0-60}$ was the highest in the MDA-MB-231 (3750) and PC-9 (3550) cell lines, followed closely by JIMT-1 (3120), and lowest in the U251 line (2760) (Table 3.3). A linear regression of peak bioluminescence times and normalized $\mathrm{AUC}_{0-60}$ resulted in an $\mathrm{r}^{2}$ of 0.52 . A one-way ANOVA showed significant differences between these curves, with the MDA-MB-231 AUC significantly different from the JIMT-1 and U251 AUCs, the JIMT-1 AUC showing significant difference to MDA-MB-231 and PC-9 AUCs, and the U251 AUC showing statistically significant difference from the MDA-MB-231 and PC-9 AUCs ( $\mathrm{p}<0.05)$ (Fig. 3.5).

\section{In-vitro two-phase decay $K_{\text {fast }}$ values correlate with peak in-vivo signal times}

After plotting radiance and normalized values against time for each in-vitro experiment, two-phase decay kinetics were utilized to measure decay until plateau ( $\left.\mathrm{K}_{\text {fast }}\right)$ and decay from plateau $\left(\mathrm{K}_{\text {slow }}\right)$, to elucidate if decay constants correlated with peak invivo luminescence time. The largest radiance $\mathrm{K}_{\text {fast }}$ was seen in the $\mathrm{U} 251$ model (0.4717), the smallest in the PC-9 model (0.1810), with MDA-MB-231 (0.3142) and JIMT-1 (0.3830) falling in between (Table 3.2). A linear regression produced an $r^{2}$ of -0.53 , indicating larger $\mathrm{K}_{\text {fast }}$ values are correlated with faster peak bioluminescence timing. The largest normalized $\mathrm{K}_{\text {fast }}$ was seen in the $\mathrm{U} 251$ model $(0.4717)$ and the slowest in the 
MDA-MB-231-Br model (0.1352), which was followed by JIMT-1-Br (0.1652) and PC-9

(0.1790) (Table 3.3). The values of normalized $\mathrm{K}_{\text {fast }}$ were plotted against peak in-vivo bioluminescence times resulting in a linear regression producing an $\mathrm{r}^{2}$ of 0.66 .

\subsection{Discussion}

D-luciferin is a non-toxic marker utilized for both in-vitro and in-vitro bioluminescence measurements. By capturing photon emission and visualizing emission trends through time, longitudinal assessment of therapy efficacy can be performed. Two issues with in-vivo and in-vitro BLI collection are addressed in this study: the optimal time to initiate signal collection, and the duration in which signal will not vary significantly from peak photon emission time. Both of these issues are dependent on which type of luciferase is used for BLI data.

Different types of luciferase exist. The three most commonly used are Rluc, Gaussia Gluc, Fluc, the latter is also termed luc2 [21, 22]. Rluc and Gluc metabolize coelenterazine to give off an intense burst of light before rapidly returning to nearbaseline levels, termed "flash kinetics" [23]. This makes Rluc and Gluc difficult to work with, as small differences in imaging time can cause significant quantification differences. In contrast, Fluc metabolizes D-luciferin to produce light and slowly decreases in signal intensity, termed "glow kinetics" [23]. Though Fluc does not produce as intense an initial signal as Rluc or Gluc, it is the most common vector for luciferin BLI due to the maintenance of signal intensity.

Photons produced by Fluc are away from the blue absorption values of Rluc $(\lambda \max =480 \mathrm{~nm})$ and Gluc $(\lambda \max =460 \mathrm{~nm})[22,24,25]$. This wavelength range overlaps with fluorescence with structural proteins such as collagen and elastin, as well as 
enzymes such as NADH and NADPH, and other proteins such as lipofuschin [26, 27]. Tissue often displays "auto-fluorescence", which can generate significantly obstructive background signal [28]. The production of photons during in-vivo fluorescence brain imaging is often attenuated by the cranium [29]. In order to quantitatively image the brain, thinning of the cranium and a window placement is often required, though data gathered may not be specific unless used in near-infrared ranges [30]. This is not optimal for brain metastatic models especially during longitudinal studies due to the number of tumors produced and the invasiveness of the procedure. The spectra range of photon emission further supports the use of Fluc over Rluc or Gluc as longer wavelengths $(>600$ $\mathrm{nm})$, especially those in the red and far-red spectrum, are better able to transmit through tissue compared to blue and green spectra [31]. Efforts are made to create Fluc analogs that produce spectra even closer to the $700 \mathrm{~nm}$ range, reaching edges of infra-red wavelengths $[32,33]$.

Of interest, we were able to fit, and apply, our data to a linear exponential decay curve. The exponential equation $\mathrm{X}_{\mathrm{t}}{ }^{*} \mathrm{e}^{-\mathrm{kt}}$ is utilized in many disciplines, including radioactivity, pharmacological half-life, and fluorescence [34]. In this equation, $X_{t}$ is a quantity at time $t$, and $\mathrm{k}$ is a value indicating growth or decay [34]. Adapted for bioluminescence, the equation becomes $\mathrm{P}_{\mathrm{t}}{ }^{*} \mathrm{e}^{-\mathrm{kt}}$, where $\mathrm{P}_{\mathrm{t}}$ and $\mathrm{k}$ represents the amount of photons/second at time $t$. In vivo, k can represent absorption $(\alpha)$ or elimination (ß); invitro this can represent fast $\left(\mathrm{K}_{\text {fast }}\right)$ or slow $\left(\mathrm{K}_{\text {slow }}\right)$ elimination. Visualized, $\mathrm{k}$ is represented by the slope connecting data points. Thus, the steeper the slope, the faster the rate constant. 
The timing of signal capture onset is essential, as seen by the large, rapid increase of photon production occurring within the first 10 minutes after luciferin injection in all models. This timing is crucial whether working with the "flash" Gluc and Rluc luciferin reporters due to their rapid onset-offset characteristics. Signal discrepancy from peak BLI is the lowest during the 10- to 20-minute time block, following an ebb-and-flow pattern as signal returns to baseline; this time block thus represents the optimal time for BLI acquisition as maximum signal will be captured. The time to reach peak BLI is generally delayed in the metastatic models compared to the intracranial model, but have nearly double optimal imaging collection periods.

It is possible that BLI kinetics of the intracranial model is due the larger number of cells injected and/or the type of tumor lesion formed. While the 150,000-175,000 cells administered in the intracardiac metastatic model produce more numerous, small lesions [35], the 500,000 cell intracranial model creates one large tumor [36]. The size of tumor may also account for the narrow optimal imaging time and the large minimal-error window, as a larger tumor may utilize more luciferin in a given period of time, as evidenced by the $\mathrm{U} 251$ radiance $\mathrm{P}_{\max }$ and AUC. This is the impetus for data normalization, as each animal between and within models is placed on the same scale for easier and more accurate comparison [37].

Entry to the brain tissue is restricted due to the presence of the blood-brain barrier (BBB). The BBB is composed of endothelial cells attached by tight junctions, astrocytic foot processes, and enzymes to efflux and degrade molecules [38]. As cancer cells grow, they often create new vasculature in a process called angiogenesis. These immature vessels are compromised, and the blood-tumor barrier (BTB) allows some permeation of 
restricted molecules into tumor and brain tissue [38]. Permeability of a substance through the BBB or BTB can be estimated using Lipinski's Rule of 5s, or visualized and quantified using radioisotope data.

Lipinski's Rule of 5 are used to estimate if molecules are able to permeate the blood-brain barrier (BBB). An optimal molecule should have a molecular weight lower than 500 daltons, lipophilicity measured by $\log P>5$, not donate more than 5 hydrogen bonds, and not accept more than 10 hydrogen bonds. [39] It should be noted that most BBB-permeant molecules have a $\log P$ of approximately 2.5 [40]. D-luciferin has a molecular weight of $280 \mathrm{Da}$, donates 2 hydrogen bonds, accepts 5 hydrogen bonds, and has a $\log P$ of 0.5 , fulfilling three of these four rules [41]. A variant of $\log P, \log D$ is calculated using a buffered solution and represents a molecule's lipophilicity at $\mathrm{pH}$ 7.4. As this mimics physiological conditions, $\log D$ may be more useful than $\log P$ in determining the BBB-penetrating capability of a molecule. A $\log D$ of $1-4$ indicates likely BBB permeability [42]. D-luciferin has a $\log D$ of approximately -2.34 at physiologic $\mathrm{pH}$, indicating high water solubility, which corresponds with the report of radioisotope ${ }^{14} \mathrm{C}-\mathrm{D}$ luciferin accumulating at comparatively low amounts into brains of naïve nude mice, the same type used in these experiments [43].

The association between vasculature and BLI is well documented. Antiangiogenic agents cause both a significant delay in peak timing as well as reduced levels of photon emission [20]. This is confirmed by visualizing reduced blood flow by magnetic resonance imaging [20]. Additionally, this study found up to $60 \%$ variability between consecutive imaging points, with a $95 \%$ confidence interval near peak showing $\leq 20 \%$ variability, which are similar to our findings [20]. A VEGFR-luc transgenic stroke 
model showed that increased new vessel formation was associated with increased photon emission, confirmed with MRI imaging showing enhanced T2 values [44]. An intracranial glioblastoma model found that luciferin BLI intensity increased as tumor angiogenesis occurred [45], and luciferin has been used for ex vivo imaging in avascular tissue such as intervertebral disks [46].

Heterogeneity of tumor vasculature may be responsible for the differences in delivery of D-luciferin and the kinetic curves. The U251 tumor vasculature is more permeable to the small ${ }^{14} \mathrm{C}$-aminoisobutyric acid molecule compared to metastatic models). Using luciferin for in-vitro experiments removes dependencies of absorption and vasculature on in-vivo BLI. Maximum photon emission begins directly after addition of D-luciferin. This is seen in the PC-9 and U251 models which then stabilized to a plateau, the timing of which is similar to their in-vivo peak time. Rather than beginning signal capture immediately after D-luciferin administration, for minimized error and optimal collection, in-vitro models should be imaged upon reaching a signal plateau, which generally resembles in-vivo peak time.

Both radiance and normalized $\mathrm{AUC}_{0-60}$ is correlated to peak bioluminescence timing. The $\mathrm{U} 251$ model had the smallest $\mathrm{AUC}_{0-60}$ but the fastest peak time, while the MDA-MB-231-Br model had the highest $\mathrm{AUC}_{0-60}$ and most delayed peak time. This suggests that despite same Fluc incorporation, cells metabolize luciferin at different rates, supported by significant differences in in-vivo peak times (highest in MDA-MB-231, lowest in U251) and in-vitro $\mathrm{K}_{\text {fast }}$ values (lowest in MDA-MB-231, highest in U251).

In conclusion, we characterized luciferin bioluminescence curves in brain tumor models and their in-vitro counterparts, highlighting intra-model similarities and inter- 
model differences. Future studies will focus on determining vascularity differences of preclinical brain metastases and primary glioblastoma, Fluc copy numbers of each cell line, and enzyme kinetics based on different routes of D-luciferin administration. 


\subsection{Conclusion}

We report the luciferin pharmacokinetics for emerging brain metastases models and an established intracranial brain tumor model, but there are three key differences. Peak bioluminescence occurs later, optimal imaging time is longer, and time of minimized error is shorter in the metastatic model. In-vitro imaging times exhibit firstorder kinetics in which signal plateau corresponds with in-vivo peak timing, revealing consistent models. The time to in-vitro plateau and in-vivo peak signal production is unique to each cell line and cannot be substituted without incurring significant error. These parameters are critical to consider as the preclinical models are used in translational efficacy studies. 


\subsection{References}

1. D'Andrea, G., et al., Brain Metastases: Surgical Treatment and Overall Survival. World Neurosurg, 2017. 97: p. 169-177.

2. McNeill, K.A., Epidemiology of Brain Tumors. Neurol Clin, 2016. 34(4): p. 981998.

3. Perkins, A. and G. Liu, Primary Brain Tumors in Adults: Diagnosis and Treatment. Am Fam Physician, 2016. 93(3): p. 211-7.

4. McFaline-Figueroa, J.R. and E.Q. Lee, Brain Tumors. Am J Med, 2018.

5. Jost, S.C., et al., Measuring brain tumor growth: combined bioluminescence imaging-magnetic resonance imaging strategy. Mol Imaging, 2009. 8(5): p. 24553.

6. Leblond, F., et al., Pre-clinical whole-body fluorescence imaging: Review of instruments, methods and applications. J Photochem Photobiol B, 2010. 98(1): p. $77-94$.

7. O'Farrell, A.C., et al., Non-invasive molecular imaging for preclinical cancer therapeutic development. Br J Pharmacol, 2013. 169(4): p. 719-35.

8. Zhu, Y., et al., Quantum dot-based nanoprobes for in vivo targeted imaging. Curr Mol Med, 2013. 13(10): p. 1549-67.

9. $\quad$ Evans, M.S., et al., A synthetic luciferin improves bioluminescence imaging in live mice. Nat Methods, 2014. 11(4): p. 393-5.

10. Kim, J.E., S. Kalimuthu, and B.C. Ahn, In vivo cell tracking with bioluminescence imaging. Nucl Med Mol Imaging, 2015. 49(1): p. 3-10. 
11. Shinde, R., J. Perkins, and C.H. Contag, Luciferin derivatives for enhanced in vitro and in vivo bioluminescence assays. Biochemistry, 2006. 45(37): p. $11103-$ 12.

12. Lomakina, G.Y., Y.A. Modestova, and N.N. Ugarova, Bioluminescence assay for cell viability. Biochemistry (Mosc), 2015. 80(6): p. 701-13.

13. Aswendt, M., et al., Boosting bioluminescence neuroimaging: an optimized protocol for brain studies. PLoS One, 2013. 8(2): p. e55662.

14. Schipper, M.L., M.R. Patel, and S.S. Gambhir, Evaluation of firefly luciferase bioluminescence mediated photodynamic toxicity in cancer cells. Mol Imaging Biol, 2006. 8(4): p. 218-25.

15. Burgos, J.S., et al., Time course of bioluminescent signal in orthotopic and heterotopic brain tumors in nude mice. Biotechniques, 2003. 34(6): p. 1184-8.

16. Inoue, Y., et al., Timing of imaging after d-luciferin injection affects the longitudinal assessment of tumor growth using in vivo bioluminescence imaging. Int J Biomed Imaging, 2010. 2010: p. 471408.

17. Inoue, Y., et al., Comparison of subcutaneous and intraperitoneal injection of Dluciferin for in vivo bioluminescence imaging. Eur J Nucl Med Mol Imaging, 2009. 36(5): p. 771-9.

18. Sim, H., et al., Pharmacokinetic modeling of tumor bioluminescence implicates efflux, and not influx, as the bigger hurdle in cancer drug therapy. Cancer Res, 2011. 71(3): p. 686-92.

19. Zinn, K.R., et al., Noninvasive bioluminescence imaging in small animals. ILAR J, 2008. 49(1): p. 103-15. 
20. Zhao, D., et al., Antivascular effects of combretastatin A4 phosphate in breast cancer xenograft assessed using dynamic bioluminescence imaging and confirmed by MRI. FASEB J, 2008. 22(7): p. 2445-51.

21. Tannous, B.A., Gaussia luciferase reporter assay for monitoring biological processes in culture and in vivo. Nat Protoc, 2009. 4(4): p. 582-91.

22. Thorne, N., J. Inglese, and D.S. Auld, Illuminating insights into firefly luciferase and other bioluminescent reporters used in chemical biology. Chem Biol, 2010. 17(6): p. 646-57.

23. Held, P., Luciferase Measurements using the Clarity Luminescence Microplate Reader - Luminescence made easy. Nature Methods Application Notes, 2006.

24. Kuchimaru, T., et al., A luciferin analogue generating near-infrared bioluminescence achieves highly sensitive deep-tissue imaging. Nat Commun, 2016. 7: p. 11856.

25. Shifera, A.S. and J.A. Hardin, Factors modulating expression of Renilla luciferase from control plasmids used in luciferase reporter gene assays. Anal Biochem, 2010. 396(2): p. 167-72.

26. Shi, L., et al., Label-Free Fluorescence Spectroscopy for Detecting Key Biomolecules in Brain Tissue from a Mouse Model of Alzheimer's Disease. Sci Rep, 2017. 7(1): p. 2599.

27. Ramanujam, N., Fluorescence spectroscopy of neoplastic and non-neoplastic tissues. Neoplasia, 2000. 2(1-2): p. 89-117. 
28. Spitzer, N., G.S. Sammons, and E.M. Price, Autofluorescent cells in rat brain can be convincing impostors in green fluorescent reporter studies. J Neurosci Methods, 2011. 197(1): p. 48-55.

29. Jung, J.C., et al., In vivo mammalian brain imaging using one- and two-photon fluorescence microendoscopy. J Neurophysiol, 2004. 92(5): p. 3121-33.

30. Hong, G., et al., Through-skull fluorescence imaging of the brain in a new nearinfrared window. Nat Photonics, 2014. 8(9): p. 723-730.

31. Negrin, R.S. and C.H. Contag, In vivo imaging using bioluminescence: a tool for probing graft-versus-host disease. Nat Rev Immunol, 2006. 6(6): p. 484-90.

32. Liang, Y., P. Walczak, and J.W. Bulte, Comparison of red-shifted firefly luciferase Ppy RE9 and conventional Luc2 as bioluminescence imaging reporter genes for in vivo imaging of stem cells. J Biomed Opt, 2012. 17(1): p. 016004.

33. Mezzanotte, L., et al., A novel luciferase fusion protein for highly sensitive optical imaging: from single-cell analysis to in vivo whole-body bioluminescence imaging. Anal Bioanal Chem, 2014. 406(23): p. 5727-34.

34. Ahmed, A., Pharmacokinetics of Drugs Following IV Bolus, IV Infusion, and Oral Administration. 2015.

35. Adkins, C.E., et al., Characterization of passive permeability at the blood-tumor barrier in five preclinical models of brain metastases of breast cancer. Clin Exp Metastasis, 2016. 33(4): p. 373-83.

36. Baumann, B.C., et al., Stereotactic intracranial implantation and in vivo bioluminescent imaging of tumor xenografts in a mouse model system of glioblastoma multiforme. J Vis Exp, 2012(67). 
37. Klerk, C.P., et al., Validity of bioluminescence measurements for noninvasive in vivo imaging of tumor load in small animals. Biotechniques, 2007. 43(1 Suppl): $\mathrm{p}$. $7-13,30$.

38. Lockman, P.R., et al., Heterogeneous blood-tumor barrier permeability determines drug efficacy in experimental brain metastases of breast cancer. Clin Cancer Res, 2010. 16(23): p. 5664-78.

39. Mikitsh, J.L. and A.M. Chacko, Pathways for small molecule delivery to the central nervous system across the blood-brain barrier. Perspect Medicin Chem, 2014. 6: p. 11-24.

40. Pajouhesh, H. and G.R. Lenz, Medicinal chemical properties of successful central nervous system drugs. NeuroRx, 2005. 2(4): p. 541-53.

41. ChemSpider, Firefly luciferin.

42. Rankovic, Z., CNS drug design: balancing physicochemical properties for optimal brain exposure. J Med Chem, 2015. 58(6): p. 2584-608.

43. Berger, F., et al., Uptake kinetics and biodistribution of 14C-D-luciferin--a radiolabeled substrate for the firefly luciferase catalyzed bioluminescence reaction: impact on bioluminescence based reporter gene imaging. Eur J Nucl Med Mol Imaging, 2008. 35(12): p. 2275-85.

44. Adamczak, J.M., et al., In vivo bioluminescence imaging of vascular remodeling after stroke. Front Cell Neurosci, 2014. 8: p. 274.

45. Sun, A., et al., Firefly luciferase-based dynamic bioluminescence imaging: a noninvasive technique to assess tumor angiogenesis. Neurosurgery, 2010. 66(4): p. 751-7; discussion 757 . 
46. Peeters, M., et al., Bioluminescence-mediated longitudinal monitoring of adiposederived stem cells in a large mammal ex vivo organ culture. Sci Rep, 2015. 5: p. 13960. 


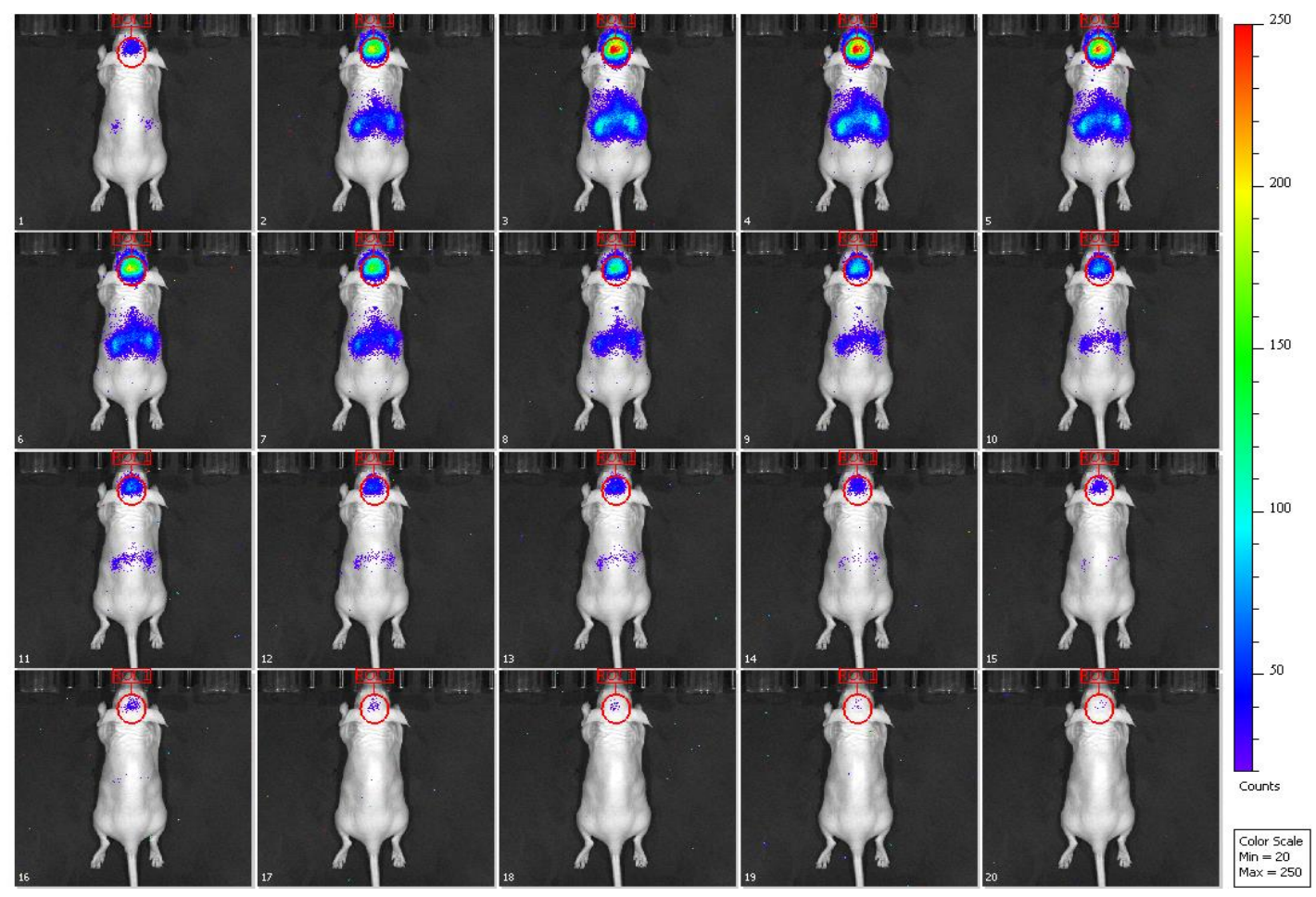

Figure 3.1. Representative time-course of bioluminescence capture and quantification.

$150 \mathrm{mg} / \mathrm{kg}$ of d-luciferin was administered via intraperitoneal injection into a mouse bearing the MDA-MB-231 brain-seeking tumor line. A region of interest was drawn around the skull to quantify photon emission. Counts are displayed as low (blue) to high (red). Data was normalized to peak values of each animal and plotted against time. The peak value can be seen at 20 minutes (Panel 4), with optimal imaging time occurring between 15 (Panel 3) and 25 minutes (Panel 6). 
a
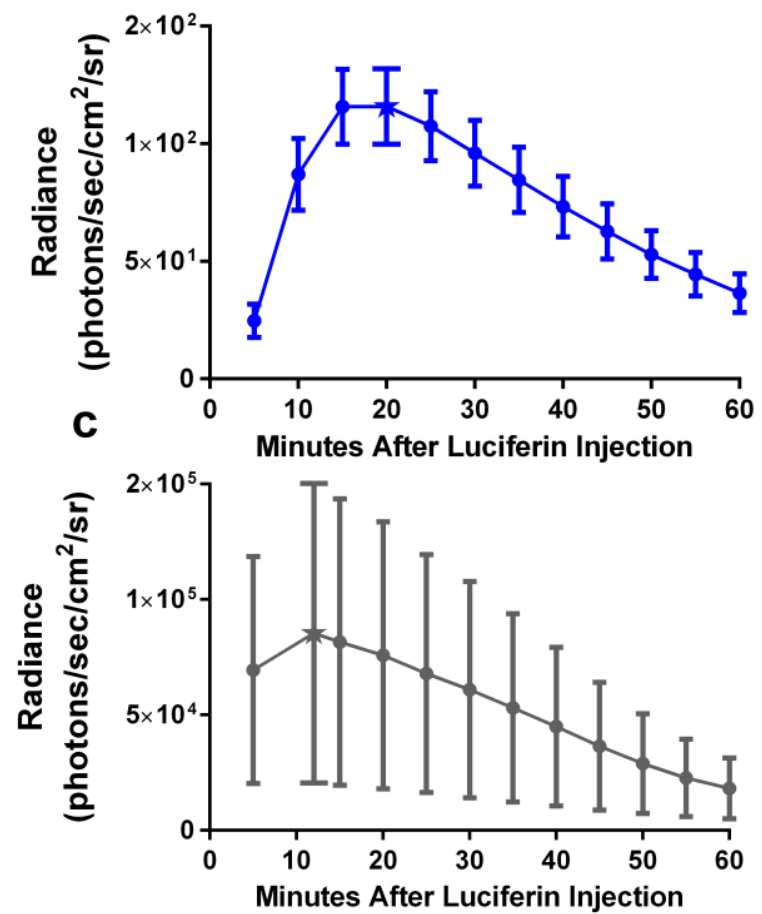

b
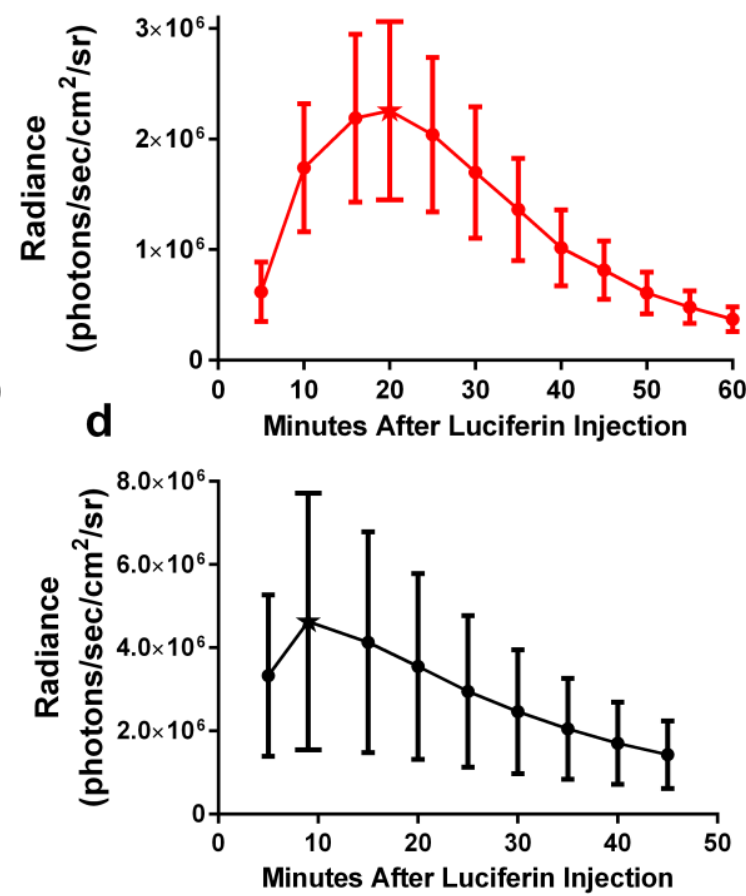

Figure 3.2. Radiance luciferin pharmacokinetics for brain tumor models.

Photons emitted per second were plotted over time in the (A) MDA-MB-231-Br, (B) JIMT-1-Br, (C) PC-9-Br, and (D) U251 models. Large variability exists between animals in the same group. Peak signal production is distinguished as a star-shape in each graph. All data points represent mean \pm SD $(n=3-4)$. 

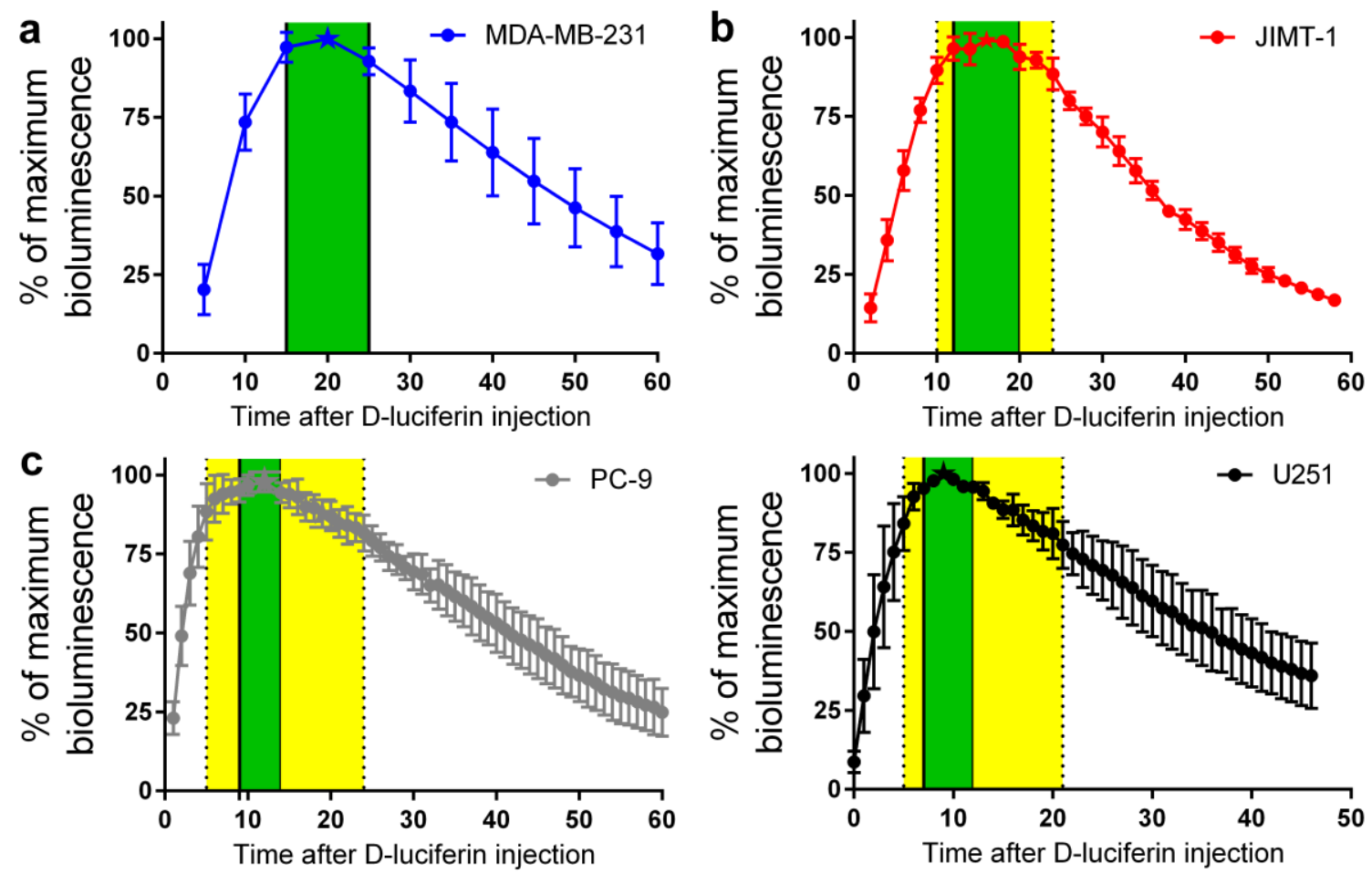

Figure 3.3. Normalized luciferin bioluminescence profiles for brain tumor models.

(A) The metastatic breast lines MDA-MB-231 and (B) JIMT-1 have delayed peak times but longer optimal imaging windows, while the (C) PC-9-Br and (D) U251 models feature the fastest onset to peak times and smallest optimal imaging window time. Green represents optimal signal capturing time (above $95 \%$ of peak) while yellow represents values that are insignificant $(\mathrm{p}<0.05)$ from peak intensity but fall outside of the optimal range. Peak BLI is distinguished as a star-shape in each graph. Data was analyzed using one-way ANOVA with Dunnet's multiple comparison test. All data points represent mean \pm SD $(n=3-4)$. 

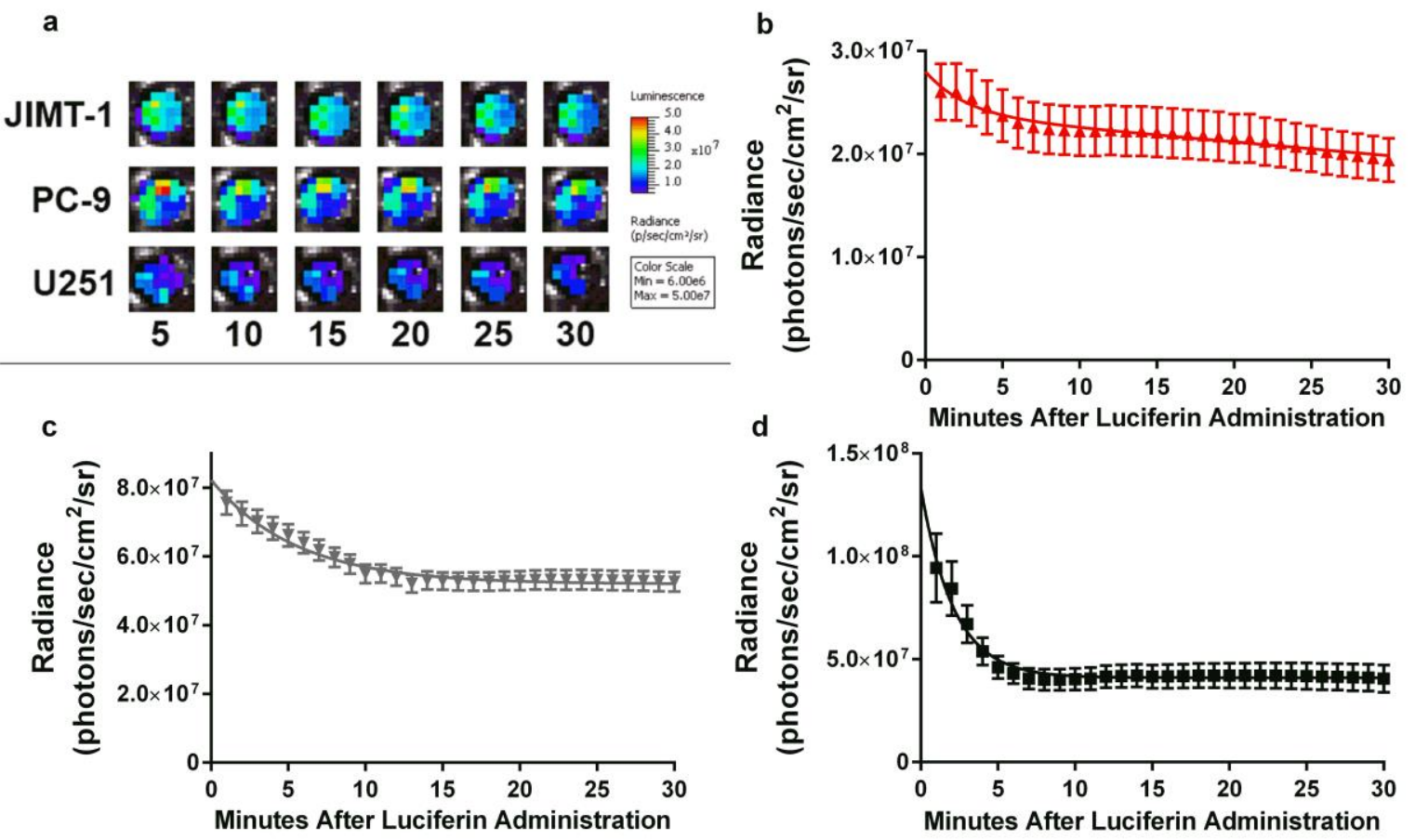

Figure 3.4. In vitro luciferin kinetics corresponds with in vivo bioluminescence.

Qualitative 5-minute imaging for imaging duration is shown for models (A). The JIMT-1 (B), PC-9 (C), and U251 (D) models display first-order kinetics with a rapid followed by a slow elimination phase. Plateau was defined as a $\pm 1 \%$ change from previous signal. In vitro models reach signal plateau at similar times to their in vivo peak times (inset arrows). All data points represent mean $\pm \operatorname{SD}(n=96)$, with error $\leq 1 \%$ residing within the plot. 


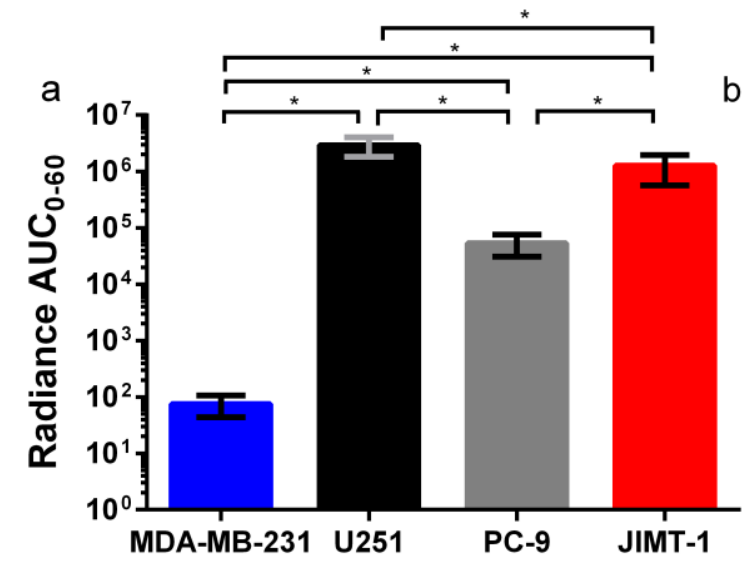

b
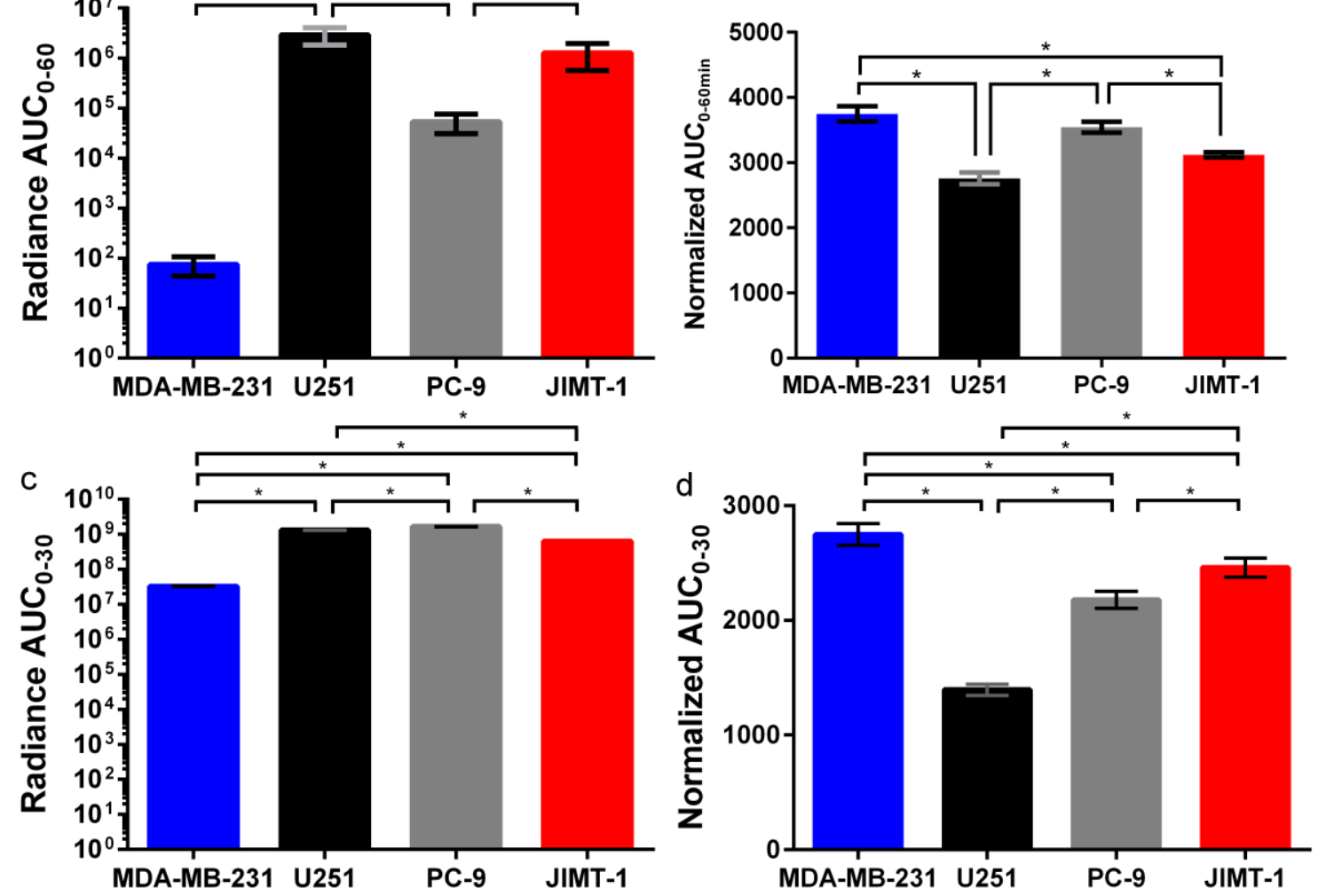

Figure 3.5. Radiance and normalized AUC comparisons.

In vivo radiance (A) and normalized (B) bioluminescence AUCs were calculated, as were in vitro radiance $(\mathbf{C})$ and normalized (D) bioluminescence AUCs for comparison. In both models, U251 shows the highest radiance AUC and lowest normalized AUC. A one-way ANOVA using Dunn's multiple comparisons showed AUC within a plot are significantly different from each other $(\mathrm{p}<0.05)$ except for normalized in vivo AUCs. Error $\leq 1 \%$ resides within the plot. All data represents mean $\pm S D, n=3-4$. 


\begin{tabular}{|l|c|c|c|c|c|}
\hline $\begin{array}{l}\text { Cell } \\
\text { Line }\end{array}$ & $\begin{array}{r}\text { Number of } \\
\text { animals }\end{array}$ & $\begin{array}{c}\text { Method of } \\
\text { Injection }\end{array}$ & $\begin{array}{c}\text { Peak } \\
\text { bioluminescence } \\
\text { time (minutes) }\end{array}$ & $\begin{array}{c}\text { Minimal error } \\
\text { imaging timing } \\
\text { (minutes) }\end{array}$ & $\begin{array}{c}\text { Time > 95\% of peak } \\
\text { bioluminescence } \\
\text { (minutes) }\end{array}$ \\
\hline $\begin{array}{l}\text { MDA- } \\
\text { MB- } \\
\text { Br }\end{array}$ & 4 & Intracardiac & 20 & $15-25$ & $15-25$ \\
\hline $\begin{array}{l}\text { JIMT-1- } \\
\text { Br }\end{array}$ & 3 & Intracardiac & 16 & $10-24$ & $12-20$ \\
\hline PC-9-Br & 4 & Intracardiac & 12 & $5-24$ & $9-14$ \\
\hline U251 & 3 & Intracranial & 9 & $5-21$ & $7-12$ \\
\hline
\end{tabular}

Table 3.1. Timing of luciferin imaging for in vivo cancer cell models.

Bioluminescence imaging for each cell line were characterized for time to peak signal value, signal statistically insignificant ( $\mathrm{p}$ $>0.05$ ) from peak value defined as minimal error time, and time above $95 \%$ of peak value defined as optimal imaging time. Metastatic models feature delayed peak timing compared to the intracranial model. Lengthier BLI peak times are associated with shorter minimal error time but longer optimal imaging time. An ANOVA with Dunn's multiple comparison test was performed to determine minimal error times, significance defined at $\mathrm{p}<0.05$ 


\begin{tabular}{|c|c|c|c|c|c|c|}
\hline $\begin{array}{l}\text { Cell } \\
\text { Line }\end{array}$ & $\begin{array}{l}\text { Radiance } \\
\text { Absorption } \\
\text { Slope }(\alpha)\end{array}$ & $\begin{array}{l}P_{\max } \pm \\
\text { SD }\end{array}$ & $\begin{array}{l}\text { Radiance } \\
\text { Elimination } \\
\text { Slope (B) }\end{array}$ & $\begin{array}{l}\text { Radiance } \\
\text { AUC } \pm \\
\text { SEM }\end{array}$ & $\begin{array}{l}\text { Radiance } \\
\mathrm{K}_{\text {fast }}\end{array}$ & $\begin{array}{l}\text { Radiance } \\
\mathrm{K}_{\text {slow }}\end{array}$ \\
\hline $\begin{array}{l}\text { MDA- } \\
\text { MB- } \\
231- \\
\mathrm{Br}\end{array}$ & 0.2161 & $\begin{array}{r}1.16 \pm \\
0.32 \\
\times 10^{2}\end{array}$ & 0.0127 & $\begin{array}{c}4.35 \pm 0.31 \\
\times 10^{3}\end{array}$ & 0.3142 & 0.1352 \\
\hline $\begin{array}{r}\text { JIMT- } \\
1-B r\end{array}$ & 0.0919 & $\begin{array}{r}2.25 \pm \\
1.34 \\
\times 10^{6}\end{array}$ & 0.0190 & $\begin{array}{c}7.35 \pm 1.04 \\
\times 10^{7}\end{array}$ & 0.3830 & 0.1652 \\
\hline $\begin{array}{l}\text { PC-9- } \\
\text { Br }\end{array}$ & 0.3689 & $\begin{array}{r}8.52 \pm \\
1.29 \\
\times 10^{5}\end{array}$ & 0.0128 & $\begin{array}{c}2.99 \pm 1.03 \\
\times 10^{6}\end{array}$ & 0.1810 & 0.1790 \\
\hline U251 & 0.1476 & $\begin{array}{r}4.61 \pm \\
5.31 \\
\times 10^{6}\end{array}$ & 0.0220 & $\begin{array}{c}1.19 \pm 0.34 \\
\times 10^{8}\end{array}$ & 0.4717 & 0.4717 \\
\hline
\end{tabular}

Table 3.2. Radiance pharmacokinetic data for tumor models.

BLI signal was plotted as radiance (photons $/ \mathrm{sec} / \mathrm{cm}^{2} /$ steridian) against time. One-phase kinetic modeling was used to plot in vivo signal rate to peak $(\alpha)$ and elimination rate $(\beta)$, and two-phase kinetic modeling for as in vitro fast $\left(\mathrm{K}_{\text {fast }}\right)$ and slow $\left(\mathrm{K}_{\text {slow }}\right)$ signal deterioration. Area under the curve (AUC) represents total photon emission during signal capture. The peak radiance (Pmax), AUC, and in vitro kinetics were highest in the intracranial U251 model while $\beta$ was lowest. A one-way ANOVA using Dunn's multiple comparison showed the AUC of each model was statistically different from one another $(\mathrm{p}<0.05)$. All data is based on $\mathrm{n}$ of 3-4 per model. 


\begin{tabular}{|l|l|l|l|l|l|c|}
\hline Cell Line & Normalized & Normalized & Normalized & Normalized curve & Normalized & Normalized \\
& Absorption & Elimination & AUC \pm SEM & difference $(\mathrm{p}<0.05)$ & $\mathrm{K}_{\text {fast }}$ & $\mathrm{K}_{\text {slow }}$ \\
\hline MDA-MB- & 0.2017 & 0.0053 & $3749 \pm 118$ & JIMT-1, U251 & 0.1279 & 0.1279 \\
Slope $\alpha)$ & Slope $(\beta)$ & & & & \\
\hline JIMT-1-Br & 0.1054 & 0.0192 & $3120 \pm 40$ & MDA-MB-231, PC-9 & 0.1971 & 0.0119 \\
\hline PC-9-Br & 0.4862 & 0.0036 & $3545 \pm 83$ & JIMT-1, U251 & 0.1817 & $1.46 \times 10^{-16}$ \\
\hline U251 & 0.2980 & 0.0137 & $2757 \pm 89$ & MDA-MB-231, PC-9 & 0.4713 & $1.17 \times 10^{-16}$ \\
\hline
\end{tabular}

Table 3.3. Normalized pharmacokinetic data for tumor models.

Signal captured during BLI was normalized to percent based on peak and plotted against time to create a normalized pharmacokinetic profile for each tumor model. In contrast to radiance data, the intracranial U251 model has the largest $\alpha$ and AUC, while the MDA-MB-231 model has the largest AUC. A one-way ANOVA using Dunn's multiple comparison showed statistically different AUC between certain models ( $\mathrm{p}<0.05)$. All data is based on $\mathrm{n}$ of 3-4 per model. 


\begin{tabular}{|c|l|l|l|l|l|l|}
\hline Cell Line & $0-10$ & $10-20$ & $20-30$ & $30-40$ & $40-50$ & $50-60$ \\
\hline $\begin{array}{l}\text { MDA-MB- } \\
\text { 231-Br }\end{array}$ & $53.2 \%$ & $26.5 \%$ & $16.5 \%$ & $19.5 \%$ & $17.6 \%$ & $14.6 \%$ \\
\hline JIMT-1-Br & $75.2 \%$ & $4.3 \%$ & $23.8 \%$ & $27.7 \%$ & $17.5 \%$ & $8.1 \%$ \\
\hline PC-9-Br & $73.7 \%$ & $9 \%$ & $15.3 \%$ & $15.5 \%$ & $14.5 \%$ & $10.6 \%$ \\
\hline U251 & $78.9 \%$ & $17.5 \%$ & $21.0 \%$ & $15.9 \%$ & $9.2 \%$ & \\
\hline
\end{tabular}

Table 3.4. Signal range during bioluminescence imaging.

After signal acquisition, data was normalized and plotted. Percent range of 10 minute blocks was calculated. The highest range occurs in the first 10 minutes in all models, followed by the 10-20-minute block which has the lowest range in most models. Potential error is greatest if imaging in the first 10 minutes, but minimal in the 10-20-minute block, corresponding with minimal-error and optimal imaging times. 


\section{CHAPTER 4}

\section{EFFECTS OF ETIRINOTECAN PEGOL (NKTR-102) IN THREE}

\section{PRECLINICAL BREAST CANCER BRAIN METASTASES MODELS}

\subsection{Introduction}

Breast cancer is both the second-most diagnosed cancer and second-most cause of cancer death in women resulting in an estimated 250,000 new cases and 40,000 deaths in 2017. [1] Brain metastases occurs in approximately 10-20\% of all breast cancer cases. [2] Prognosis is typically poor and often dependent on metastatic lesion number, size and cell phenotype. [3] Triple negative breast cancer (TNBC) and human epidermal growth factor receptor (HER2)-positive metastases occur more frequently, numerously, and are associated with the shortest survival. [3-5] Treatment of breast cancer brain metastasis (BCBM) includes surgical resection, whole brain radiation therapy (WBRT), stereotatic radiation, and systemic and targeted chemotherapy. [6, 7] TNBC makes up approximately $10-15 \%$ of breast cancer cases and 20\% of BCBM [8] while HER2+ makes up approximately $20 \%$ of breast cancer cases and up to half of patients develop brain metastases. $[9,10]$ Despite advances in treatment, survival after diagnosis of BCBM ranges between 4 and 26 months. [3-5] The resistance of BCBM to chemotherapy is mainly due to the physiochemical activities of the blood-brain barrier (BBB) and bloodtumor barrier (BTB). [4, 5]

The physical BBB is composed of endothelial cells joined by tight junctions, a basement membrane, pericytes, and astrocytic foot processes. [11] Efflux transporters such as P-glycoprotein (Pgp), breast cancer resistance protein (BCRP), and intracellular 
enzymes (phosphatases, oxidases) comprise the chemical portion of the BBB, further restricting brain penetration of chemotherapy. $[11,12]$ In brain metastases, vasculature is often compromised, resulting in the BTB. Though often described as "leaky", vascular disruption in the BTB does not always significantly impact chemotherapeutic penetrance due to intact efflux activity. $[13,14]$ Pharmaceutical development of chemotherapy that offers enhanced brain penetration often consists of active agents conjugated to polymers like polyethylene glycol (PEG). [15]

Etirinotecan pegol consists of four arms in which each arm is composed of a PEG polymer, a cleavable ester linker, and a molecule of irinotecan. As a $20 \mathrm{kDa}$ molecule, penetration of etirinotecan pegol into normal tissue is limited by intact vasculature but has less difficulty penetrating tumor vasculature. $[16,17]$ Upon administration, ester cleavage slowly releases irinotecan which is further metabolized to the active $\mathrm{SN}-38$ molecule, providing sustained topoisomerase I inhibitory activity. [18] Etirinotecan pegol has shown efficacy as monotherapy in chemotherapy-resistant metastatic ovarian [19] and breast cancer [20]. The BEACON trial did not show significant survival difference of etirinotecan pegol against physician's choice (eribulin, vinorelbine, gemcitabine, paclitaxel or docetaxel) in the treatment of advanced breast cancer [16] but post-hoc analysis of data showed that BCBM patients could benefit from therapy. [21]

As previously reported, etirinotecan pegol dosed at $50 \mathrm{mg} / \mathrm{kg}$ equivalents of irinotecan in a preclinical triple negative $\mathrm{BCBM}$ model provides $\mathrm{SN}-38$ concentrations similar to the $145 \mathrm{mg} / \mathrm{m}^{2}$ dose used in the BEACON trial, which lead to improved survival against placebo and conventional irinotecan $50 \mathrm{mg} / \mathrm{kg}$. [17] Adapting the intent of the BEACON trial, we hypothesized that this dose of etirinotecan pegol would 
significantly improve survival when compared to eribulin, vinorelbine, gemcitabine, or docetaxel monotherapy in the human triple negative MD-MBA-231-BrLuc, murine triple negative 4T1-BrLuc, and human HER2+ JIMT1-BrLuc BCBM models.

Herein, we present evidence that etirinotecan pegol $50 \mathrm{mg} / \mathrm{kg}$ preferentially accumulates into metastatic lesions compared to non-tumor brain parenchyma. Etirinotecan pegol increases median survival in both MDA-MB-231-BrLuc and JIMT-1BrLuc human breast-brain metastases models $(\mathrm{p}<0.05)$ compared to traditional physician's choice chemotherapy ( $p>0.05$ ), but does not improve survival in the murine 4T1 model $(\mathrm{p}>0.05)$.

\subsection{Methods and Materials}

\section{Animals}

Female athymic nude mice aged 4-8 weeks weighing 20-25 grams were bought from Charles River Laboratory (Kingston, NY) and housed for a minimum of 72 hours to facilitate acclimation prior to intracardiac injection. Mice were housed in microisolator cages with a 12-hour light/dark cycle and received sterilized food and water ad libitum in accordance with West Virginia University's Animal Care and Use Committee (ACUC protocol 13-1207). Upon reaching survival endpoints animals were euthanized by ketamine $(100 \mathrm{mg} / \mathrm{kg})$ and xylazine $(8 \mathrm{mg} / \mathrm{kg})$ mixture. All animal experiments were performed according to the principles of the Guide for the Care and use of Laboratory animals.

\section{Cells}


Firefly luciferase transfected into the human metastatic brain-seeking MDA-MB231-BrLuc cell line, and was kindly provided by Dr. Patricia Steeg from the National Institutes of Health (NIH). Similar transfection was done to JIMT1 cells to create the JIMT1-BrLuc line, and the 4T1 line to create the 4T1-BrLuc line. Cells were kept at $37^{\circ}$ in 5\% $\mathrm{CO}_{2}$, grown in Dulbecco's Modified Eagle Medium (DMEM) supplemented with $10 \%$ fetal bovine serum and 5\% penicillin-streptomycin. All cells used for in vivo and in vitro experiments were between passages 5 to 10 .

\section{In-vitro toxicology}

5,000 cancer cells per well were seeded into a 96-well plate. Chemotherapy was dissolved in negligible amounts of DMSO and prepared in media. For consistency, the same amount of DMSO was added to control media. After addition of chemotherapy, plates were incubated for 72 hours. On endpoint, $50 \mu \mathrm{g} / 50 \mu \mathrm{L}$ of MTT was added to each

well and allowed to incubate for a further 2 hours. Wells were then aspirated and $100 \mathrm{uL}$ of DMSO was added to each well for to solubilize the purple formazan crystals. After gentle shaking for an hour, wells were analyzed for absorbance at $570 \mathrm{~nm}$. Concentration curves were plotted and $\mathrm{IC}_{50}$ values were calculated using a four-phase non-linear analysis.

\section{Stereotactic intracardiac injection}

Mice were induced and maintained by inhaling $2 \%$ isoflurane anesthesia and placed in a stereotactic apparatus (Stoelting). 1.75x10 $0^{5}$ MDA-MB-231-BrLuc, JIMT1BrLuc, or 4T1 cells in $100 \mathrm{uL}$ of PBS were measured by an Invitrogen Countess I 
(ThermoFischer) and then injected into the left ventricle by approximation or ultrasound guidance. [17]

\section{Drugs and dosing}

Gemcitabine, docetaxel, and vinorelbine were acquired from SelleckChem. Eribulin was acquired from Adooq BioSciences. Lapatinib was acquired from ApexBio. Etirinotecan pegol was provided by Nektar Therapeutics. Saline was chosen as the vehicle. Gemcitabine, vinorelbine and etirinotecan pegol were dissolved in saline. Docetaxel was dissolved in 5\% ethanol/5\% Tween $80 / 90 \%$ saline. Eribulin was dissolved in 5\% ethanol/15\% distilled water/80\% saline. Vinorelbine $10 \mathrm{mg} / \mathrm{kg}[22,23]$, docetaxel $10 \mathrm{mg} / \mathrm{kg}$ [24-26], and etirinotecan pegol $50 \mathrm{mg} / \mathrm{kg}$ equivalent of irinotecan were dosed every 7 days via tail vein; eribulin $1.5 \mathrm{mg} / \mathrm{kg}$ [27] and Gemcitabine $60 \mathrm{mg} / \mathrm{kg}$ [28] were injected every 4 days via intraperitoneal injection. Lapatinib was suspended in $0.1 \%$ Tween 80 and 1\% hydroxypropylmethylcellulose in water, dosed orally twice daily at $100 \mathrm{mg} / \mathrm{kg}$. [29] Dosing was initiated on Day 21 (Week 3) for the MDA-MB-231-BrLuc group, on Day 14 (Week 2) for the JIMT1-BrLuc group, and on Day 3 for the 4T1-BrLuc group (Table 4.1). All doses were made fresh on the day of administration and administered approximately half an hour after luciferin imaging.

\section{Imaging and Survival}

Animals were given a $150 \mathrm{mg} / \mathrm{kg}$ injection of D-luciferin potassium salt (PerkinElmer, Waltham, MA) via intraperitoneal injection and placed into $2 \%$ isoflurane for sedation. After 10-15 minutes to ensure luciferin circulation, animals were then transferred to the IVIS Lumina II (PerkinElmer) and bioluminescence imaging (BLI) was 
performed. BLI was taken twice weekly to measure tumor burden. Regions of interest (ROI) were drawn based on cranial circumference and BLI is reported as radiance (photons $/ \mathrm{sec} / \mathrm{cm}^{2} /$ steradian).

\section{Autoradiography}

Animals were anesthetized and given $10 \mu \mathrm{Ci}$ of ${ }^{14} \mathrm{C}$-etirinotecan pegol via femoral vein injection. Mice were allowed to recover while the radioactive drug circulated for 24 hours. At the endpoint, mice were again anesthetized and the descending aorta was clamped. Phosphate buffer saline was infused via left ventricle at $5 \mathrm{~mL} / \mathrm{min}$ for 2 minutes to provide a washout. Brains were then extracted and frozen to be sliced in $20 \mu \mathrm{m}$ slices, then placed in autoradiography cassettes to develop for 21 days. Cresyl violet stains were used to trace tumor areas, which were then overlaid on autoradiographic slides for use by MCID software for analysis.

\section{Statistics}

Survival analysis was plotted on a Kaplan-Meier curve and statistically compared using log-rank statistics. All results and statistical analysis were performed on GraphPad Prism 6.0, and were considered statistically significant at $\mathrm{p}<0.05$.

\subsection{Results}

In-vitro sensitivity to $\mathrm{SN}-38$ correlates with in-vivo survival efficacy

To determine sensitivity of cell lines to BEACON chemotherapy, MTT toxicology was performed as outlined in the methods and materials. The MDA-MB-231BrLuc line was the most sensitive to NKTR-102's parent drug irinotecan (1 $\mu \mathrm{M} \pm 0.11$ $\mu \mathrm{M})$ and active metabolite $\mathrm{SN}-38(10.5 \mathrm{nM} \pm 2.9 \mathrm{nM})$ compared to the 4T1-BrLuc (12.7 
$\mu \mathrm{M} \pm 0.47 \mu \mathrm{M}$ and $24.9 \mathrm{nM} \pm 0.06 \mathrm{nM}$, respectively) and JIMT-1-BrLuc (39 $\mu \mathrm{M} \pm 1.78$

$\mu \mathrm{M}$ and 222.8 $\mathrm{nM} \pm 0.17 \mathrm{nM}$, respectively). The results are summarized in Table 4.2.

\section{Etirinotecan pegol prolongs survival of human triple negative and HER2+ breast cancer brain metastases, but not the murine triple negative model}

To evaluate our main aim in comparing the efficacy of our investigational drug to established chemotherapeutics, we injected MDA-MB-231-BrLuc, 4T1-Br, and JIMT1BrLuc cells as described. Tumor growth was monitored using bioluminescence (BLI) twice weekly starting on day 21 (Week 3) post-injection in the 231-Br line (Fig. 4.1A), 14 days (Week 2) in the JIMT1-Br line (Fig. 4.3A), and 3 days in the 4T1-Br line (Fig. 4.5A). In 231-Br vehicle animals, tumor burden increased nearly 1000-fold from baseline (Fig. 4.1B) resulting in a median survival of 40 days (Fig. 4.2). Similarly, animals treated with gemcitabine in this cell line had bioluminescence increase nearly 1000-fold (Fig. 4.1B), but survived longer (median survival 68 days, Fig. 4.2). Eribulin, docetaxel and vinorelbine treated animals had tumor burden increase nearly 100-fold with median survivals as 40.5, 39, and 43 days, respectively (Fig. 4.2). No animals in the 231-Br vehicle or conventional chemotherapy group survived beyond day 60. Etirinotecan pegol treated mice in the 231-Br model had bioluminescence plateau 10-fold above baseline (Fig. 4.1B) and median survival was 86 days (Fig. 4.2) with four mice (40\%) surviving until the end of the trial. In the JIMT1 model, dosing was initiated on day 14. Vehicle burden peaked a maximum of 10,000-fold from baseline (Fig. 4.2B) with a median survival of 28 days (Fig. 4.4). Bioluminescence in lapatinib mice peaked at 100-fold above baseline (Fig. 4.2B) with a median survival of 29 days (Fig. 4.3B). Etirinotecan pegol closely followed the bioluminescence curve of lapatinib in this model, but like in 
the 231-Br group, prevented further increases in signal (Fig. 4.2B) with a median survival of 34 days (Fig. 4.4). In the 4T1-Br group, both NKTR-102 and conventional chemotherapy had similar bioluminescence curves aside from gemcitabine, which was significantly right-shifted but eventually reached similar maximum values (Fig. 4.5B). Survival corresponded with the bioluminescence curve for 4T1-Br, as gemcitabine's median survival (28.5 days) was nearly double that of vehicle (15 days) and most other conventional chemotherapy agents (Fig. 4.6). The results are summarized in Table 4.3.

\section{Etirinotecan pegol accumulates preferentially in tumor tissue compared to brain} parenchyma

To evaluate the distribution of etirinotecan pegol, $10 \mu \mathrm{Ci}$ of ${ }^{14} \mathrm{C}$-labeled drug was administered and circulated for 24 hours. After brain extraction, $20 \mu \mathrm{m}$ slices placed in autoradiography cassettes and developed for 21 days. Cresyl violet staining was performed to outline tumor areas (Fig. 4.7A). Quantification of 20 tumors revealed that

${ }^{14} \mathrm{C}$-etirinotecan pegol accumulated to $2603 \pm 917 \mathrm{ng} / \mathrm{g}$ in tumor tissue and $220 \pm 40 \mathrm{ng} / \mathrm{g}$ in control regions of brain (Fig. 4.7B), more than a 10-fold difference (Fig. 4.7C).

\subsection{Discussion}

The principal finding of the present experiment was that etirinotecan pegol significantly improved survival compared to vehicle and conventional chemotherapy groups in the human MDA-MB-231-BrLuc and JIMT-1-BrLuc groups, but not the murine 4T1-BrLuc group. In the MDA-MB-231-BrLuc group, etirinotecan pegol administration prolonged survival beyond double of any conventional chemotherapy group with nearly half of treated mice surviving until the end of the 90-day trial. Similarly, etirinotecan administration led to one mouse surviving in the JIMT-1 group 
until the end of the trial. No conventional chemotherapy treatment mice of any cell line survived until the end of the trial. In both the MDA-MB-231-Br and JIMT-1-Br lines, etirinotecan pegol shows early control of tumor burden based on bioluminescence, which was sustained in the 231-Br model. The results of this study correspond with findings in our previous trial with etirinotecan pegol in the 231-Br model [17].

The timing of chemotherapeutic administration is crucial to its effects on survival. In order to prevent metastases, chemotherapy has to be given while cancer cells are still circulating or initially attaching to parenchyma, typically before two weeks in preclinical models [30]. In our model, metastases have been established and allowed to grow for up to three weeks prior to chemotherapy administration, mimicking clinical settings. Though lapatinib has shown efficacy in reducing lesion size and improving survival in HER2 BCBM settings, the JIMT-1 cell line is intrinsically resistant to the drug and represents a pre-treated model, explaining why the drug did not improve survival compared to the vehicle in this setting $[30,31]$.

The 11-fold greater accumulation of ${ }^{14} \mathrm{C}$-etirinotecan pegol into tumor tissue compared to non-tumor parenchyma demonstrates that the drug is able to traverse the disrupted BTB to create its sustained effects. This replicates findings of the previous study using autoradiographic data in which ${ }^{14} \mathrm{C}$-etirinotecan pegol significantly and preferentially accumulated in brain metastases compared to non-tumor regions [17]. As expected, the sustained depot-like effects of pegylating irinotecan allowed for sustained SN-38 delivery to tumors, which led to superior tumor burden control as evidenced by a plateau in BLI in the 231-Br and JIMT-1 models. 
Conventional irinotecan has typically not been used as part of a therapeutic regimen for $\mathrm{BCBM}$, and has not demonstrated any significant improvements in increasing median survival in preclinical settings. [17, 32] However, liposomal irinotecan increased median survival by 13 days at equivalent doses to conventional irinotecan [32]. Additionally, liposomal irinotecan was found to increase and prolong the exposure of the active SN-38 metabolite compared to conventional irinotecan, which corresponds to the findings of the previous etirinotecan pegol study $[17,32]$. This sustained exposure to SN38 is documented clinically in two Phase 1 [33] and two Phase 2 etirinotecan trials [19, 20], and has led to liposomal irinotecan involvement in a clinical trial (NCT01770353).

The discrepancy of NKTR-102 efficacy may be due to the origin of cell lines used. MDA-MB-231-BrLuc and JIMT-1 are human epithelial derived lines, whereas 4T1-BrLuc is a murine derived line. Since NKTR-102 is a topoisomerase I inhibitor used in human cancer, it is possible that the administration of the drug did not have any significant effect in the murine model.

Another possibility is NKTR-102 did not penetrate as readily into 4T1-Br tumors as it does in 231-Br tumors. While both cell lines create tumors in the brain, 4T1 tumors are more numerous and smaller, whereas 231 tumors are larger in size and fewer in number [34]. The smaller size and reduced vascularity of 4T1 tumors could restrict the large 20kDa NKTR-102 molecule from penetrating and effectively reducing tumor burden. This is not problematic for gemcitabine as it is a small molecule and readily uptaken by tumor cells due to its resemblance as a nucleotide, which may explain gemcitabine's efficacy in the 4T1 model [35]. 
Alternatively, the efficacy of etirinotecan pegol may be due to tumor aggressiveness. While the MDA-MB-231 and JIMT-1 lines take 14-21 days postintracardiac injection to establish brain metastases, the 4T1 line is quicker to form and grow tumors, with most treatment groups' median survival occurring in this time period. It is possible that etirinotecan pegol works on slower-growing tumors, allowing for the release of SN-38 over time to appropriately achieve cytotoxic concentrations and effect. This phenomenon is known as enhanced permeation and retention (EPR).

Compromised tumor vasculature allows for increased permeability and allow substances to create a reserve and slowly release for days to weeks [36, 37]. Clinicallyused dosage forms exhibiting EPR include encapsulation in liposomes (Myocet®), conjugation to polyethylene glycol (Onzeald $®)$ or albumin (Abraxane $\left.{ }^{\circledR}\right)$, or a combination of both, as is the case with pegylated liposomal doxorubicin (Doxil@) [36]. Preclinical and clinical evidence has shown that these formulations improve circulation half-life and extravasate in metastatic lesions, though effects on overall survival are ambiguous [36]. With autoradiographic evidence that etirinotecan pegol deposits and is retained in tumor tissue, along with sustained irinotecan and SN-38 levels in the tumor environment, we believe that the molecule achieves its tumoricidal effects through EPR. The continuous effects of etirinotecan pegol may also lead to a preventative phenomenon, as circulating cancer cells sensitive to the agent may be killed before extravasating and developing into metastases.

Etirinotecan pegol is demonstrated to be efficacious in two preclinical models representing the most common types of $\mathrm{BCBM}$. We have observed that etirinotecan pegol provides long systemic concentrations of irinotecan and SN-38. Our radiographic 
data demonstrate etirinotecan pegol permeates through the compromised BTB and accumulates in BCBM, which we believe is responsible for the increase in survival compared to conventional chemotherapy and vehicle groups in the 231-Br and JIMT-1 models. The translational findings of this trial are significant.

As targeted and novel chemotherapy agents surge in development, survival from brain metastases of breast cancer remains poor with a prognosis between 2 to 34 months. The post-hoc clinical findings from the BEACON trial, in combination with previous preclinical findings and this preclinical trial, support the use of etirinotecan pegol as a therapeutic option in the ongoing ATTAIN trial, which evaluates its efficacy against TPC to treat brain metastases from triple-negative and HER2+ breast cancer.

\subsection{Conclusion}

Etirinotecan pegol accumulates in metastatic brain lesions and significantly improves survival compared to conventional chemotherapeutic agents in two human cellular models of triple-negative and HER2+ brain metastases of breast cancer. These data support the use of etirinotecan pegol in the Phase III ATTAIN trial.

\subsection{References}

1. DeSantis, C.E., et al., Breast cancer statistics, 2017, racial disparity in mortality by state. CA Cancer J Clin, 2017. 67(6): p. 439-448.

2. Leone, J.P. and B.A. Leone, Breast cancer brain metastases: the last frontier. Exp Hematol Oncol, 2015. 4: p. 33. 
3. Niikura, N., et al., Brain metastases in breast cancer. Jpn J Clin Oncol, 2014. 44(12): p. 1133-40.

4. Lim, E. and N.U. Lin, Updates on the management of breast cancer brain metastases. Oncology (Williston Park), 2014. 28(7): p. 572-8.

5. Kodack, D.P., et al., Emerging strategies for treating brain metastases from breast cancer. Cancer Cell, 2015. 27(2): p. 163-75.

6. Lin, X. and L.M. DeAngelis, Treatment of Brain Metastases. J Clin Oncol, 2015. 33(30): p. 3475-84.

7. Lin, N.U., et al., CNS metastases in breast cancer: old challenge, new frontiers. Clin Cancer Res, 2013. 19(23): p. 6404-18.

8. De Ieso, P.B., et al., Breast cancer brain metastases - A 12 year review of treatment outcomes. Breast, 2015. 24(4): p. 426-33.

9. Ramakrishna, N., et al., Recommendations on disease management for patients with advanced human epidermal growth factor receptor 2-positive breast cancer and brain metastases: American Society of Clinical Oncology clinical practice guideline. J Clin Oncol, 2014. 32(19): p. 2100-8.

10. Giordano, S.H., et al., Systemic therapy for patients with advanced human epidermal growth factor receptor 2-positive breast cancer: American Society of Clinical Oncology clinical practice guideline. J Clin Oncol, 2014. 32(19): p. 2078-99.

11. Blecharz, K.G., et al., Control of the blood-brain barrier function in cancer cell metastasis. Biol Cell, 2015. 107(10): p. 342-71. 
12. Wilhelm, I., et al., Role of the blood-brain barrier in the formation of brain metastases. Int J Mol Sci, 2013. 14(1): p. 1383-411.

13. Adkins, C.E., et al., P-glycoprotein mediated efflux limits substrate and drug uptake in a preclinical brain metastases of breast cancer model. Front Pharmacol, 2013. 4: p. 136.

14. Lockman, P.R., et al., Heterogeneous blood-tumor barrier permeability determines drug efficacy in experimental brain metastases of breast cancer. Clin Cancer Res, 2010. 16(23): p. 5664-78.

15. Nance, E.A., et al., A dense poly(ethylene glycol) coating improves penetration of large polymeric nanoparticles within brain tissue. Sci Transl Med, 2012. 4(149): p. 149 ra119.

16. Perez, E.A., et al., Etirinotecan pegol (NKTR-102) versus treatment of physician's choice in women with advanced breast cancer previously treated with an anthracycline, a taxane, and capecitabine (BEACON): a randomised, open-label, multicentre, phase 3 trial. Lancet Oncol, 2015. 16(15): p. 1556-68.

17. Adkins, C.E., et al., NKTR-102 Efficacy versus irinotecan in a mouse model of brain metastases of breast cancer. BMC Cancer, 2015. 15: p. 685.

18. Hoch, U., et al., Nonclinical pharmacokinetics and activity of etirinotecan pegol (NKTR-102), a long-acting topoisomerase 1 inhibitor, in multiple cancer models. Cancer Chemother Pharmacol, 2014. 74(6): p. 1125-37.

19. Vergote, I.B., et al., Randomized multicenter phase II trial comparing two schedules of etirinotecan pegol (NKTR-102) in women with recurrent platinum- 
resistant/refractory epithelial ovarian cancer. J Clin Oncol, 2013. 31(32): p. 4060-6.

20. Awada, A., et al., Two schedules of etirinotecan pegol (NKTR-102) in patients with previously treated metastatic breast cancer: a randomised phase 2 study. Lancet Oncol, 2013. 14(12): p. 1216-25.

21. Cortes, J., et al., Prolonged survival in patients with breast cancer and a history of brain metastases: results of a preplanned subgroup analysis from the randomized phase III BEACON trial. Breast Cancer Res Treat, 2017. 165(2): p. 329-341.

22. Watanabe, K., et al., Blockade of the extracellular signal-regulated kinase pathway enhances the therapeutic efficacy of microtubule-destabilizing agents in human tumor xenograft models. Clin Cancer Res, 2010. 16(4): p. 1170-8.

23. Kraus-Berthier, L., et al., Histology and sensitivity to anticancer drugs of two human non-small cell lung carcinomas implanted in the pleural cavity of nude mice. Clin Cancer Res, 2000. 6(1): p. 297-304.

24. Bradshaw-Pierce, E.L., S.G. Eckhardt, and D.L. Gustafson, A physiologically based pharmacokinetic model of docetaxel disposition: from mouse to man. Clin Cancer Res, 2007. 13(9): p. 2768-76.

25. He, X., et al., CDK2-AP1 inhibits growth of breast cancer cells by regulating cell cycle and increasing docetaxel sensitivity in vivo and in vitro. Cancer Cell Int, 2014. 14(1): p. 130. 
26. Feng, L., S.R. Benhabbour, and R.J. Mumper, Oil-filled lipid nanoparticles containing 2'-(2-bromohexadecanoyl)-docetaxel for the treatment of breast cancer. Adv Healthc Mater, 2013. 2(11): p. 1451-7.

27. Towle, M.J., et al., Broad spectrum preclinical antitumor activity of eribulin (Halaven $(R))$ : optimal effectiveness under intermittent dosing conditions. Anticancer Res, 2012. 32(5): p. 1611-9.

28. Le, H.K., et al., Gemcitabine directly inhibits myeloid derived suppressor cells in $B A L B / c$ mice bearing $4 T 1$ mammary carcinoma and augments expansion of $T$ cells from tumor-bearing mice. Int Immunopharmacol, 2009. 9(7-8): p. 900-9.

29. Gril, B., et al., Effect of lapatinib on the outgrowth of metastatic breast cancer cells to the brain. J Natl Cancer Inst, 2008. 100(15): p. 1092-103.

30. Palmieri, D., et al., Profound prevention of experimental brain metastases of breast cancer by temozolomide in an MGMT-dependent manner. Clin Cancer Res, 2014. 20(10): p. 2727-39.

31. Koninki, K., et al., Multiple molecular mechanisms underlying trastuzumab and lapatinib resistance in JIMT-1 breast cancer cells. Cancer Lett, 2010. 294(2): p. 211-9.

32. Mohammad, A.S., et al., Liposomal Irinotecan Accumulates in Metastatic Lesions, Crosses the Blood-Tumor Barrier (BTB), and Prolongs Survival in an Experimental Model of Brain Metastases of Triple Negative Breast Cancer. Pharm Res, 2018. 35(2): p. 31. 
33. Jameson, G.S., et al., A multicenter, phase I, dose-escalation study to assess the safety, tolerability, and pharmacokinetics of etirinotecan pegol in patients with refractory solid tumors. Clin Cancer Res, 2013. 19(1): p. 268-78.

34. Adkins, C.E., et al., Characterization of passive permeability at the blood-tumor barrier in five preclinical models of brain metastases of breast cancer. Clin Exp Metastasis, 2016. 33(4): p. 373-83.

35. Renier, C., et al., Regression of experimental NIS-expressing breast cancer brain metastases in response to radioiodide/gemcitabine dual therapy. Oncotarget, 2016.

36. Nichols, J.W. and Y.H. Bae, EPR: Evidence and fallacy. J Control Release, 2014. 190: p. 451-64.

37. Maeda, H., Macromolecular therapeutics in cancer treatment: the EPR effect and beyond. J Control Release, 2012. 164(2): p. 138-44. 


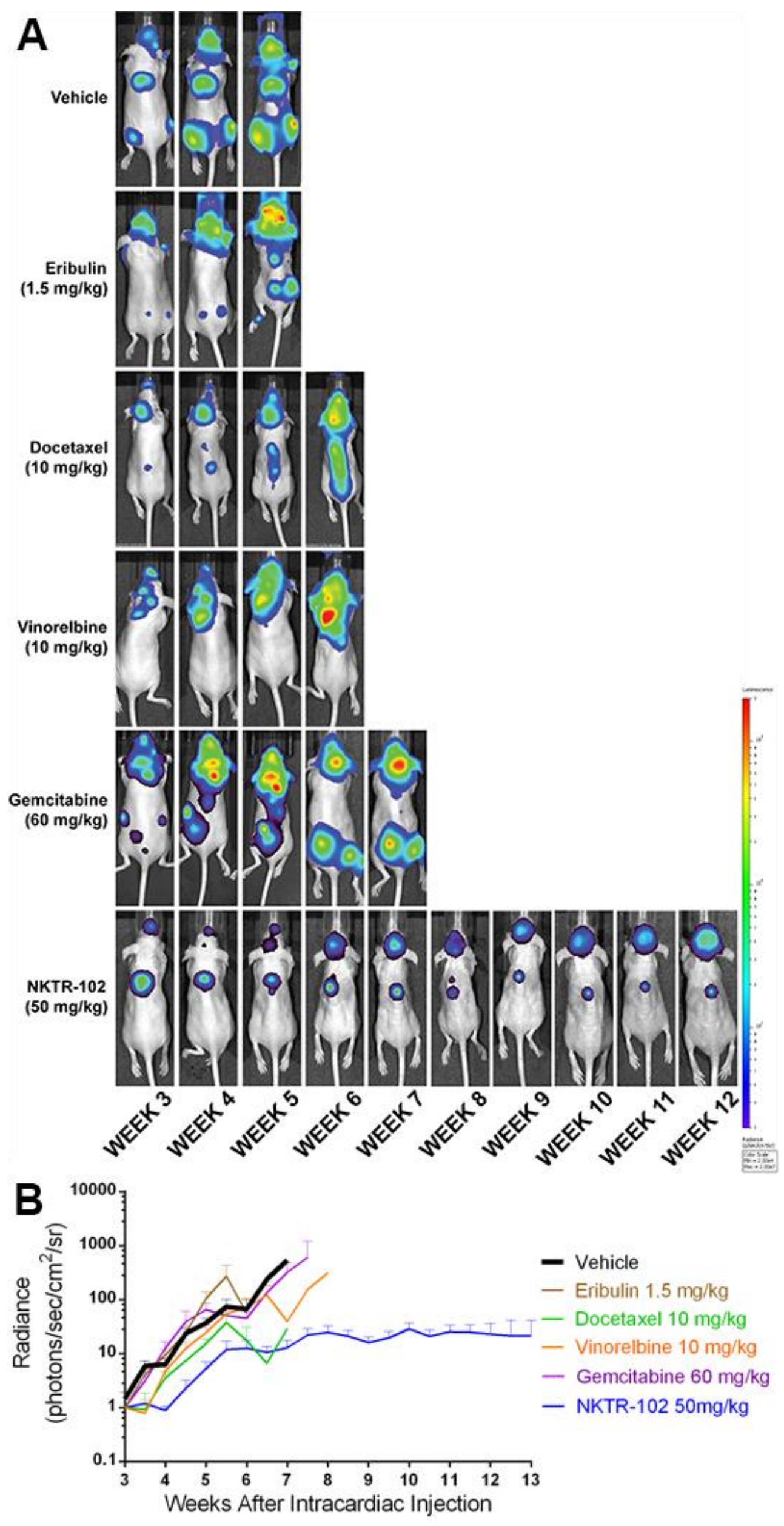


Figure 4.1. Etirinotecan pegol reduces tumor burden in the MDA-MB-231-Br model compared to conventional chemotherapy and vehicle.

(A) Following intracardiac injection of MDA-MB-231-Br cells and establishment of brain metastases, biweekly imaging on the IVIS Lumina tracked tumor growth and chemotherapeutic efficacy. Images shown are of the same animal sequentially. (B) Mean BLI signal versus time in 231-Br mice treated with therapy. Treatment was initiated on day 21. Each data point represents mean $\pm \mathrm{SD}(\mathrm{n}=2-10$ per time point $)$. Tumor burden in etirinotecan pegol-treated mice was significantly lower than conventional chemotherapy or vehicle treatment groups $(\mathrm{p}<0.05)$. 


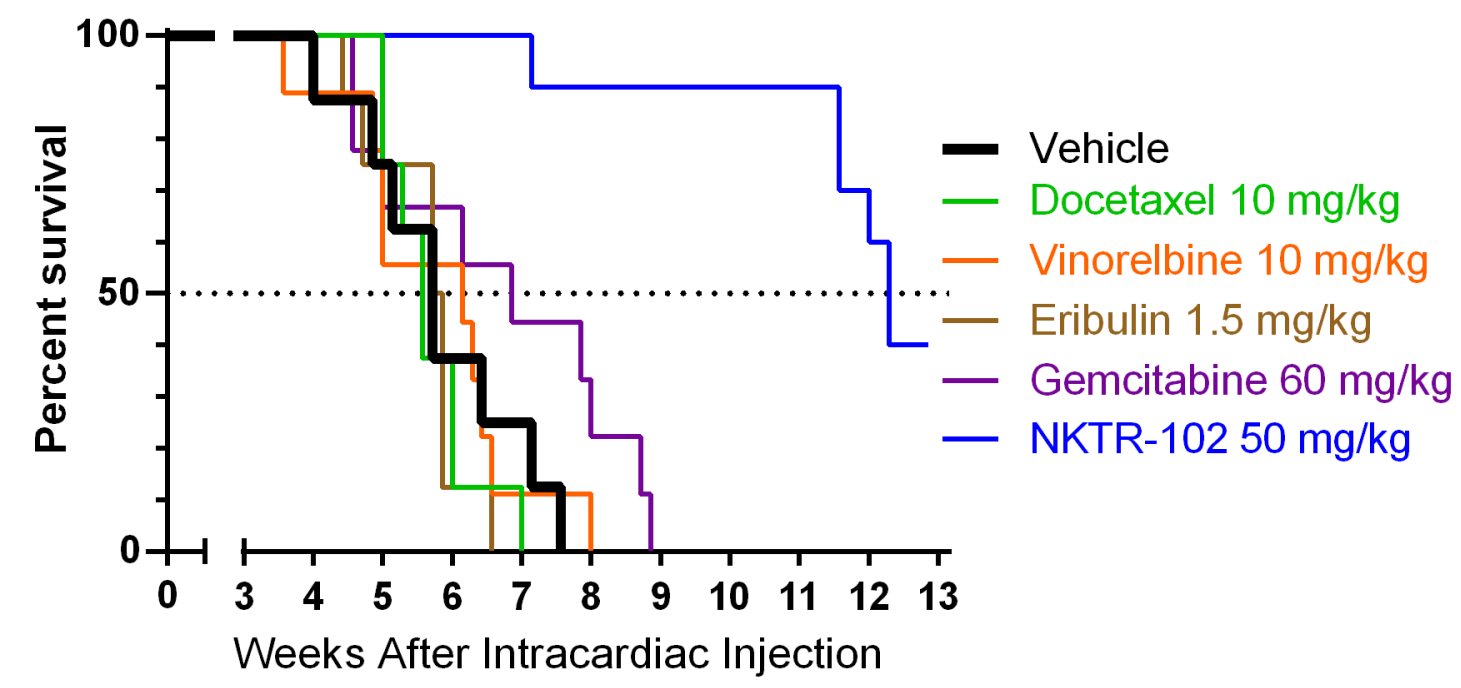

Figure 4.2. Kaplan-Meier survival curve of MDA-MB-231-Br mice treated with etirinotecan pegol and conventional chemotherapy.

On day 21 after intracardiac injection of MDA-MB-231-Br cells, mice were treated with vehicle (saline, $\mathrm{n}=8)$, docetaxel $(10 \mathrm{mg} / \mathrm{kg}, \mathrm{n}=8)$, vinorelbine $(10 \mathrm{mg} / \mathrm{kg}, \mathrm{n}=8)$, eribulin $(1.5 \mathrm{mg} / \mathrm{kg}, \mathrm{n}=8)$, gemcitabine $(60 \mathrm{mg} / \mathrm{kg}, \mathrm{n}=9)$, or etirinotecan pegol (50 $\mathrm{mg} / \mathrm{kg}, \mathrm{n}=10$ ). Median survival time were 40 days for vehicle, 39 days for docetaxel, 43 days for vinorelbine, 40.5 days for eribulin, 48 days for gemcitabine, and 86 days for etirinotecan pegol. Etirinotecan pegol significantly increased median survival in 231-Br tumor mice compared to vehicle or conventional chemotherapy, with $40 \%$ of mice surviving to the end of trial $(\mathrm{p}<0.05)$. Data was analyzed using log-rank statistics. 


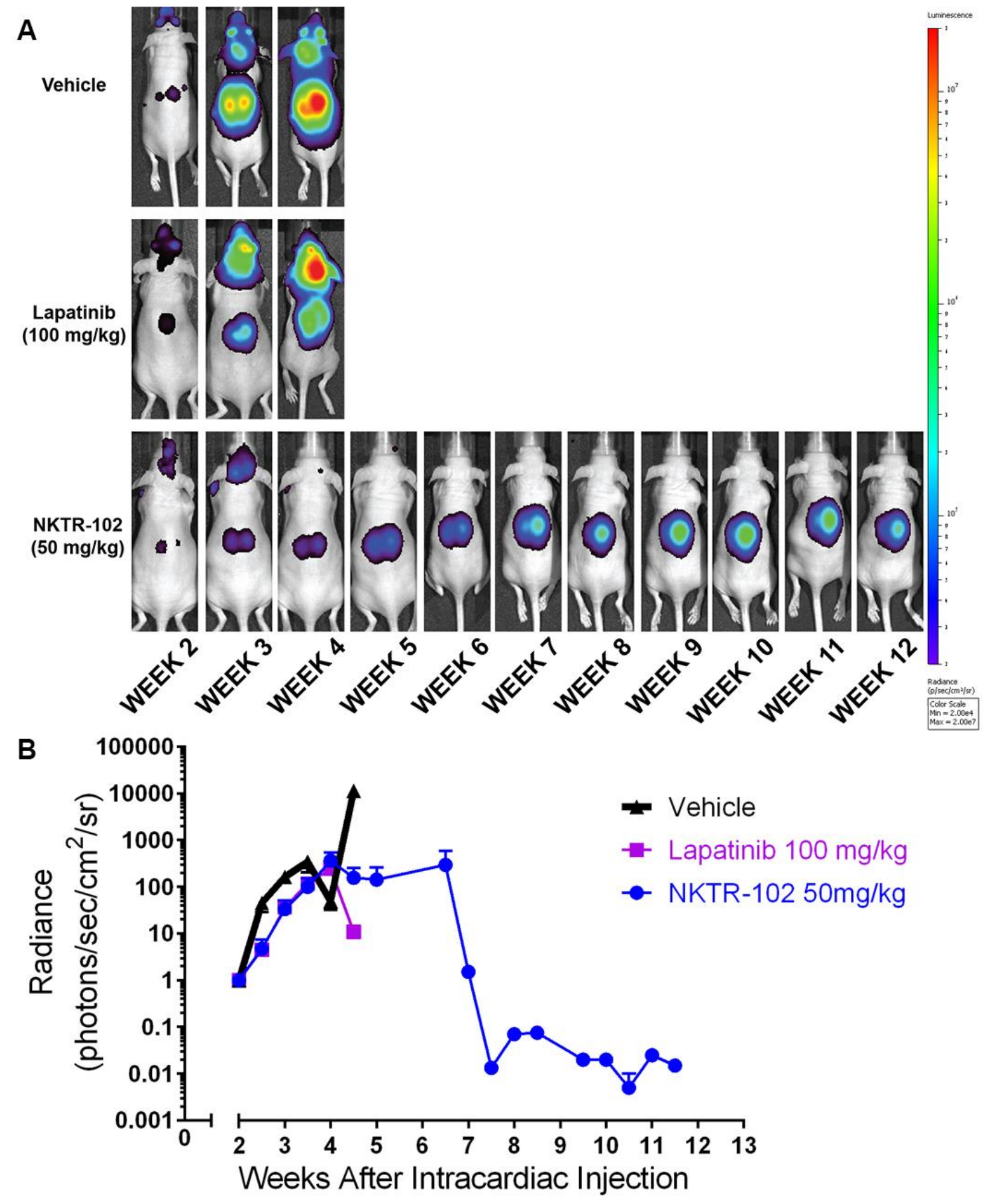


Figure 4.3. Etirinotecan pegol reduces tumor burden in the JIMT-1-Br model compared to lapatinib and vehicle.

(A) Following intracardiac injection of JIMT-1-Br cells and establishment of brain metastases, biweekly imaging on the IVIS Lumina tracked tumor growth and chemotherapeutic efficacy. Images shown are of the same animal sequentially. (B) Mean BLI signal versus time in JIMT-1-Br mice treated with therapy. Treatment was initiated on day 14. Each data point represents mean $\pm \operatorname{SD}(n=2-10$ per time point $)$. Tumor burden in etirinotecan pegol-treated mice was significantly lower than lapatinib or vehicle treatment groups $(\mathrm{p}<0.05)$. 


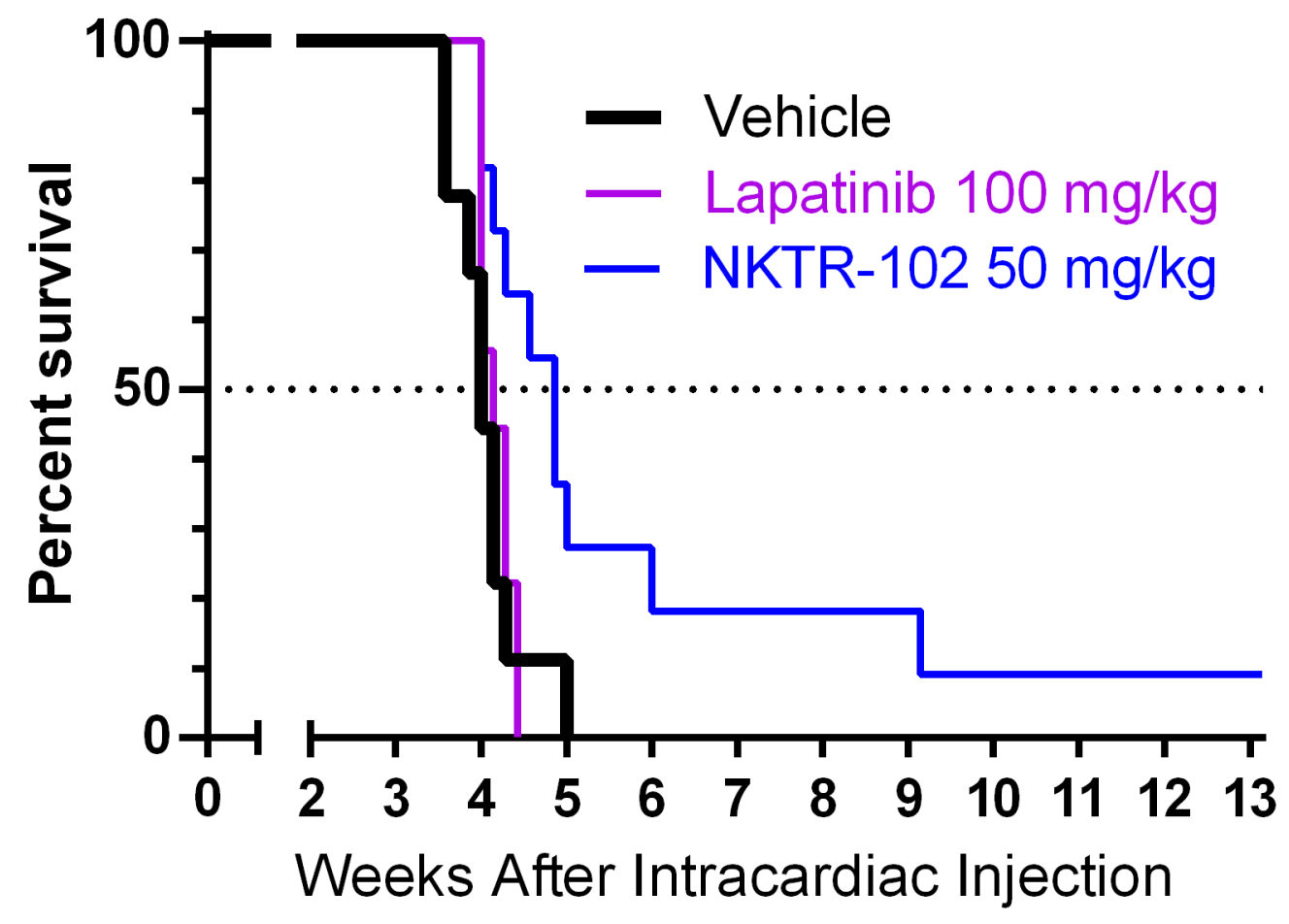

Figure 4.4. Kaplan-Meier survival curve of JIMT-1-Br mice treated with etirinotecan pegol and conventional chemotherapy.

On day 14 after intracardiac injection of JIMT-1-Br cells, mice were treated with vehicle (saline, $\mathrm{n}=9)$, lapatinib (100 mg/kg, $\mathrm{n}=9)$, or etirinotecan pegol $(50 \mathrm{mg} / \mathrm{kg}, \mathrm{n}=10)$. Median survival times were 28 days for vehicle, 29 days for docetaxel, and 34 days for etirinotecan pegol. Etirinotecan pegol significantly increased median survival in JIMT-1$\mathrm{Br}$ tumor mice compared to vehicle or conventional chemotherapy, with $10 \%$ of mice surviving to the end of trial $(\mathrm{p}<0.05)$. Data was analyzed using log-rank statistics. 

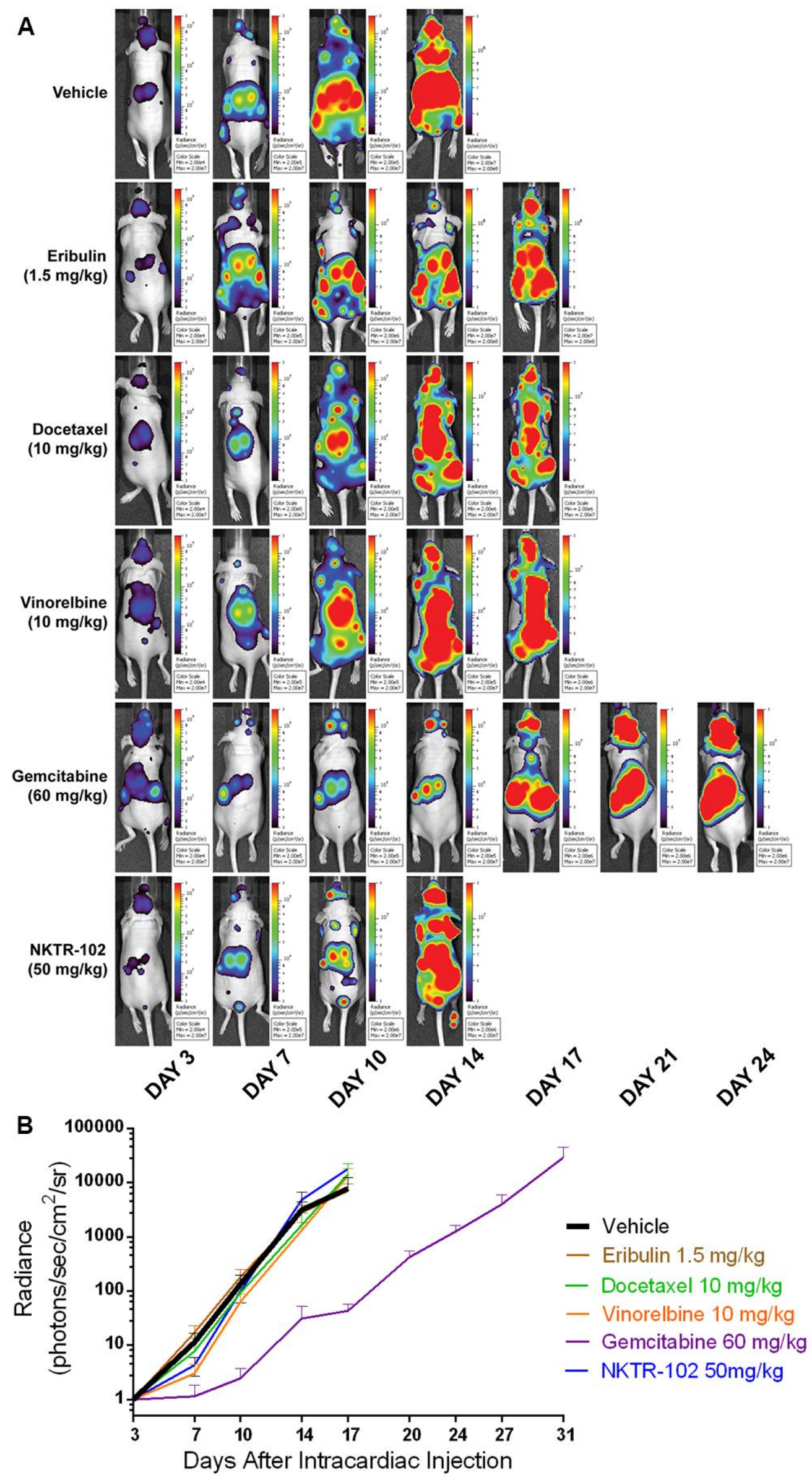
Figure 4.5. Etirinotecan pegol does not significantly reduce tumor burden in the 4T1-Br model compared to vehicle.

(A) Following intracardiac injection of 4T1-Br cells and establishment of brain metastases, biweekly imaging on the IVIS Lumina tracked tumor growth and chemotherapeutic efficacy. Images shown are of the same animal sequentially. (B) Mean BLI signal versus time in JIMT-1-Br mice treated with therapy. Treatment was initiated on day 3. Each data point represents mean $\pm \mathrm{SD}(\mathrm{n}=2-10$ per time point $)$. Tumor burden in etirinotecan pegol-treated mice was not significantly lower than vinorelbine, eribulin, or vehicle treatment groups $(\mathrm{p}>0.05)$. 


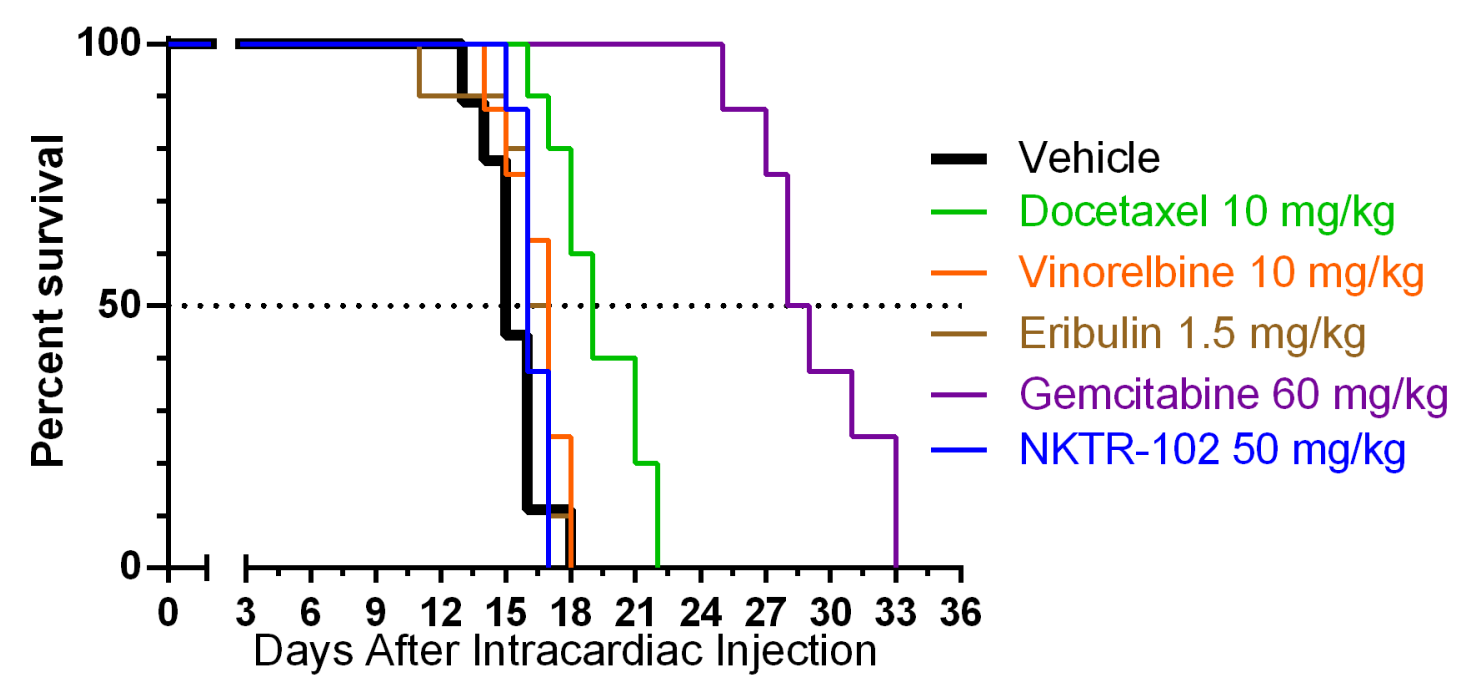

Figure 4.6. Kaplan-Meier survival curve of 4T1-Br mice treated with etirinotecan pegol and conventional chemotherapy.

On day 3 after intracardiac injection of $4 \mathrm{~T}-1-\mathrm{Br}$ cells, mice were treated with vehicle (saline, $\mathrm{n}=9)$, docetaxel $(10 \mathrm{mg} / \mathrm{kg}, \mathrm{n}=10)$, vinorelbine $(10 \mathrm{mg} / \mathrm{kg}, \mathrm{n}=8)$, eribulin $(1.5$ $\mathrm{mg} / \mathrm{kg}, \mathrm{n}=10)$, gemcitabine $(60 \mathrm{mg} / \mathrm{kg}, \mathrm{n}=8)$, or etirinotecan pegol $(50 \mathrm{mg} / \mathrm{kg}, \mathrm{n}=9)$. Median survival time were 15 days for vehicle, 19 days for docetaxel, 17 days for vinorelbine, 16.5 days for eribulin, 28.5 days for gemcitabine, and 16 days for etirinotecan pegol. Unlike gemcitabine or docetaxel, tirinotecan pegol did not significantly improve median survival compared to vehicle $(\mathrm{p}>0.05)$. Data was analyzed using log-rank statistics. 

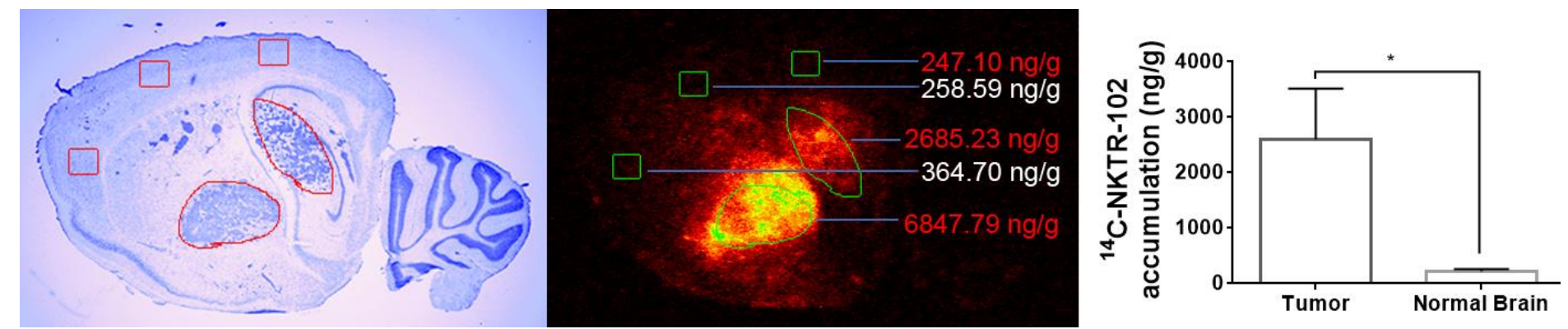

Figure 4.7. Accumulation of irinotecan in tumors and normal brain from ${ }^{14} \mathrm{C}$-etirinotecan pegol.

(A) A representative sagittal image of a MDA-MB-231-Br brain metastases and corresponding (B) ${ }^{14} \mathrm{C}$-etirinotecan pegol accumulation 24 hours after intravenous administration of radiolabeled etirinotecan pegol. (C) The mean lesion accumulation of 14 C-etirinotecan pegol was significantly higher than non-tumor regions $(\mathrm{p}<0.05)$. Data represent mean $\pm \mathrm{SD}$ of $\mathrm{n}=20$ tumors. 


\begin{tabular}{|c|c|c|c|c|}
\hline Drug & Source & Dose & Formulation & Administration \\
\hline Docetaxel & SelleckChem & $10 \mathrm{mg} / \mathrm{kg}$ & $5 \%$ ethanol, $5 \%$ Tween $80,90 \%$ saline & IV every 7 days \\
\hline $\begin{array}{l}\text { Eribulin } \\
\text { mesylate }\end{array}$ & Adooq & $1.5 \mathrm{mg} / \mathrm{kg}$ & $\begin{array}{c}5 \% \text { ethanol, } 15 \% \text { distilled water, } 80 \% \\
\text { saline }\end{array}$ & IP, every 4 days \\
\hline $\begin{array}{l}\text { Etirinotecan } \\
\text { pegol }\end{array}$ & $\begin{array}{c}\text { NEKTAR } \\
\text { Therapeutics }\end{array}$ & $50 \mathrm{mg} / \mathrm{kg}$ & Saline & IV every 7 days \\
\hline Gemcitabine & SelleckChem & $60 \mathrm{mg} / \mathrm{kg}$ & Saline & IP, every 4 days \\
\hline Lapatinib & ApexBio & $100 \mathrm{mg} / \mathrm{kg}$ & $\begin{array}{c}0.1 \% \text { Tween } 80,1 \% \\
\text { hydroxypropylmethylcellulose, } \\
\text { distilled water }\end{array}$ & PO twice daily \\
\hline Vinorelbine & SelleckChem & $10 \mathrm{mg} / \mathrm{kg}$ & Saline & IV every 7 days \\
\hline
\end{tabular}

Table 4.1. Chemotherapy formulations and dosage schedules for NKTR-102 BCBM.

IV: intravenous via tail vein, IP: intraperitoneal, PO: per os, oral gavage. 


\begin{tabular}{|l|c|l|l|}
\hline & MDA-MB-231-Br & JIMT-1-Br & 4T1-Br \\
\hline Compound & $\mathrm{IC}_{50} \pm \mathrm{SD}$ & $\mathrm{IC}_{50} \pm \mathrm{SD}$ & $\mathrm{IC}_{50} \pm \mathrm{SD}$ \\
\hline Docetaxel & $0.41 \pm 0.2 \mu \mathrm{M}$ & & \\
\hline Eribulin & $3.1 \pm 0.2 \mu \mathrm{M}$ & & $4.3 \pm \mu \mathrm{M}$ \\
\hline Gemcitabine & & & $174 \pm \mathrm{nM}$ \\
\hline Irinotecan & $1 \pm 0.11 \mu \mathrm{M}$ & $39 \pm \mu \mathrm{M}$ & $12.6 \pm \mu \mathrm{M}$ \\
\hline Lapatinib & & $1.4 \pm \mu \mathrm{M}$ & \\
\hline SN-38 & $11.2 \pm 2.9 \mathrm{nM}$ & $222 \pm \mathrm{nM}$ & $24.9 \pm \mathrm{nM}$ \\
\hline Vinorelbine & $16.4 \pm 11.2 \mathrm{nM}$ & & $10 \pm \mathrm{nM}$ \\
\hline
\end{tabular}

Table 4.2. In-vitro cytotoxicity assay of chemotherapy in cancer cell lines.

5,000 cancer cells per well were treated with increasing concentrations of compound. All data represents mean $\pm \mathrm{SD}, \mathrm{n}=96$. 


\begin{tabular}{|l|c|l|l|l|l|c|}
\hline & \multicolumn{2}{|c|}{ MDA-MB-231-Br } & \multicolumn{2}{c|}{ JIMT-1-Br } & \multicolumn{2}{c|}{ 4T1-Br } \\
\hline & $\begin{array}{c}\text { Survival } \\
\text { (Days) }\end{array}$ & vs. Vehicle & $\begin{array}{c}\text { Survival } \\
\text { (Days) }\end{array}$ & vs. Vehicle & $\begin{array}{c}\text { Survival } \\
\text { (Days) }\end{array}$ & vs. Vehicle \\
\hline Docetaxel & 39 & 0.41 & & & 18.5 & $<0.01^{*}$ \\
\hline Eribulin & 40.5 & 0.54 & & & 16.5 & 0.83 \\
\hline Gemcitabine & 48 & 0.11 & & & 26 & $<0.01^{*}$ \\
\hline Irinotecan & 42 & 0.90 & & & & \\
\hline NKTR-102 & 86 & $<0.01^{*}$ & 34 & $<0.01^{*}$ & 16 & 0.80 \\
\hline Lapatinib & & & 29 & 0.49 & & \\
\hline Vehicle & 40 & & 28 & & 16 & 0.52 \\
\hline Vinorelbine & 43 & 0.93 & & & 17 & \\
\hline
\end{tabular}

\section{Table 4.3. Survival analysis of chemotherapy and vehicle.}

Intracardiac injections resulted in triple negative (231-Br, 4T1) or HER2+ (JIMT-1) breast cancer brain metastases which were treated with chemotherapy. NKTR-102 extended median survival by 46 days in the 231-Br model, 6 days in the JIMT-1 model, but did not improve survival in the 4T1 model. 


\section{CHAPTER 5}

\section{CHARACTERIZATION OF PRECLINICAL PC-9 LUNG CANCER BRAIN METASTASES}

\subsection{Introduction}

Lung cancer is the leading cause of cancer death in both men and women worldwide. [1] Lung cancer is the most common cause of brain metastases, which is often a fatal prognosis due to a lack of curative treatment modalities. [2] There is no one universal effective screening tool for lung cancer as there are for other cancer types, such as breast cancer or melanoma. [3] The lapse between disease onset and diagnosis allows the majority of lung cancer to be metastatic on presentation. Up to $65 \%$ of all lung cancer patients will eventually develop brain metastases, making lung cancer brain metastases (LCBM) a disease with heavy burden. Though certain characteristics are known about brain metastases in general, investigation has revealed surprising information about brain metastases based on tissue of origin.

In 2010, it was discovered that breast cancer brain metastases (BCBM) featured increased vascular permeability due to loss of integrity, and that accumulation of chemotherapy is increased in metastatic lesions compared to brain parenchyma. [4] This permeability was variable within metastases, between the triple negative and HER2+ subtypes, and due to the blood-tumor barrier (BTB) restriction of chemotherapy, only approximately $10 \%$ of brain metastases were reaching cytotoxic concentrations. [4] Multiple preclinical studies since have demonstrated that permeability within and 
between $\mathrm{BCBM}$ are heterogeneous, with the conclusion that more brain-penetrating agents must be created, or methods developed to escape the barrier restriction. [5, 6]

Characterization of vascular permeability and chemotherapy uptake are yet to be performed on LCBM. The two most common types of LCBM are small-cell and nonsmall-cell lung cancer, the latter has three prominent mutations: KRAS, EGFR, and EMLA-ALK. Currently, no FDA-approved targeted therapy exists towards the most common KRAS mutation. Within the scope of EGFR, the deletion on exon 19 confers sensitivity to targeted inhibitors. Subsequently, the PC-9 cell line bearing the EGFR del 19 mutation was developed into brain-seeking metastatic lines and studied in the context of effects of passive vascular permeability and chemotherapeutic efficacy.

Herein, we use the passive permeability marker ${ }^{14} \mathrm{C}$-aminoisobutyric acid (MW 103.12), a P-glycoprotein substrate Oregon Green (MW 509.38), and albumin-bound vascularity marker indocyanine green (MW 774.96) to study effects of chemotherapy on tumor vasculature in the PC-9 model of LCBM. Permeability of these markers was studied in vehicle brains and brains treated with cisplatin and etoposide, cisplatin and pemetrexed, and the EGFR tyrosine kinase inhibitors gefitinib, afatinib, and osimertinib. We observed that the brain-seeking variant of the PC-9 line are more resistant to chemotherapy than their parenteral counterparts. Passive permeability of ${ }^{14} \mathrm{C}$-AIB was generally significantly higher in tumor regions compared to non-tumor regions. In contrast, there was no significant correlation between tumor size and ${ }^{14} \mathrm{C}$-AIB permeability. OG and ICG fold increases varied between each treatment group and were not predictable. Tumor sizes are smaller in treatment groups that extend median survival. Lastly, we observed that there was no correlation between survival and tumor size. This 
is the first paper to illustrate the heterogeneity of tumor distribution and vascular permeability of lung-brain metastases, especially in the context of therapeutic treatment.

\subsection{Methods and Materials}

\section{Animals}

The animal protocol used is the same as in Chapter 3.2.

\section{Cells}

The parenteral PC-9 cells were provided by Dr. Lori Hazlehurst's laboratory, and came transduced to display Tomato Red and Firefly luciferase (Luc2=tdT), allowing for fluorescence quantification and bioluminescence tracking. The pcDNA3.1(+)/Luc2=tdT was a gift from Christopher Contag (addgene plasmid \# 32904). Cells were grown in RPMI supplemented with $10 \%$ fetal bovine serum, $5 \%$ penicillin-streptomycin, and 10 $\mu \mathrm{L} / \mathrm{mL}$ of G4918 to ensure selection of transduced cells. Cells were kept at 37C and 5\% $\mathrm{CO}_{2}$. All cells used for in-vivo and in-vitro experiments were between passages 1-10.

\section{Stereotactic injection}

Mice were anesthetized and induced using 2\% isoflurane. After placement into a stereotactic device (Stoelting), approximately 150,000 PC-9 cells in $100 \mu \mathrm{L}$ were injected into the left ventricle. Bioluminescence verified presence in the brain. Upon termination, animals were euthanized and brains were extracted to begin ex-vivo creation of the PC-9 brain seeking line (PC-9-Br).

\section{PC9-Br-6 creation}


The protocol developed by Yoneda et al. was similarly followed to establish the PC-9-Br line. [7] After cancer cells were injected and animals became moribund with neurological symptoms, they were given $100 \mathrm{mg} / \mathrm{ketamine}$ and $8 \mathrm{mg} / \mathrm{kg}$ xylazine and then euthanized. The brain was removed from the cranium and minced into fine pieces. The minces were added to $10 \mathrm{~mL}$ of $1.5 \mathrm{mg} / \mathrm{mL}$ of collagenase I in DMEM and shaken at 300 RPM for 1 hour at 37C. The partially-digested mince was passed through a 19G syringe to further break up tissue, then strained through a $70 \mu \mathrm{m}$ cell strainer. The collagenase-containing mixture was neutralized with equal amount of DMEM and FBS for 10 minutes, then rinsed with $10 \mathrm{~mL}$ of PBS. To separate myelin from the cells, $10 \mathrm{~mL}$ of $25 \%$ bovine serum albumin in PBS was added and spun at 2000 to 2200 RPM for 15 to 20 minutes. Cells were then collected and placed immediately in media with G418 selection to allow for growth. After 3 days, cells were washed and re-plated for at least 24 hours prior to re-injection in mice. This process was repeated until the extracted population predominantly formed tumors in the brain, which was 6 times for the PC-9 line.

\section{Drugs and dosing}

Cisplatin $(5 \mathrm{mg} / \mathrm{kg}$, weekly) and either etoposide $(100 \mathrm{mg} / \mathrm{kg}$, days 2 through 5 after cisplatin administration) or pemetrexed $(100 \mathrm{mg} / \mathrm{kg}$, days 3 through 5 after cisplatin administration) were selected to represent the most common nonspecific platinum doublet therapy given to lung cancer patients. Cisplatin and pemetrexed were dissolved in saline, and etoposide was dissolved in 5\% DMSO, 5\% Tween 80, and 90\% saline prior to intravenous dosing. Gefitinib (6.25 mg/kg), afatinib (30 mg/kg), and osimertinib (25 $\mathrm{mg} / \mathrm{kg}$ ), representing three generations of EGFR inhibitors, were dosed orally once daily, 
and were formulated in $1 \%$ DMSO, $29 \%$ polyethylene glycol 400, and 70\% distilled water. All chemotherapy was purchased from SelleckChem. Drugs, formulations, and dosing regimens are summarized in Table 5.1.

\section{Brain extraction}

Upon reaching survival endpoints, mice were anesthetized and given tail vein injections of $150 \mu \mathrm{g}$ of Oregon Green (OG) dissolved in PBS, along with $10 \mu \mathrm{Ci}$ of ${ }^{14} \mathrm{C}$ aminoisobutyric acid $\left({ }^{14} \mathrm{C}\right.$-AIB). Following a 10-minute circulation, the descending aorta and inferior vena cava were clamped off. A solution of $6 \mathrm{mg}$ of indocyanine green (IR820 , ICG) bound to $0.27 \%$ bovine serum albumin (270 $\mathrm{mg}$ in $10 \mathrm{~mL})$ was perfused through the left ventricle at $5 \mathrm{~mL} / \mathrm{min}$ to provide a washout. Brains were then rapidly removed and submerged in isopentane $\left(-80^{\circ} \mathrm{C}\right)$ and stored at $-20^{\circ} \mathrm{C}$ prior to tissue slicing and visualization.

\section{Tissue processing and quantification}

Brains were mounted and $20 \mu \mathrm{m}$ slices were created with the Leica CM3050S cryotome (Leica Microsystems, Wetzlar, Germany), which were transferred to charged microscope slides. Each slide contains 3 slices for a total of approximately 120 slices per brain. Brain slice fluorescence was acquired using a stereomicroscope (Olympus MVX10; Olympus, Center Valley, PA) equipped with a 0.5 NA 2X objective and a monochromatic cooled CCD scientific camera (Retiga 4000R, QIMaging, Surrey, BC, Canada). Tomato Red fluorescence was imaged using a DsRed sputter filter (excitation/band $\lambda 545 / 25 \mathrm{~nm}$, emission/band $\lambda 605 / 70 \mathrm{~nm}$ and dichromatic mirror at $\lambda$ 565nm) (Chroma Technologies, Bellows Falls, VT), Oregon Green using an ET-GFP 
sputter filter (excitation/band $\lambda 470 / 40 \mathrm{~nm}$, emission/band $\lambda 525 / 50 \mathrm{~nm}$ and dichromatic mirror at $\lambda 495 \mathrm{~nm})$ (Chroma Technologies, Bellows Falls, VT), and ICG using a Cy7 sputter filter (excitation/band $\lambda 710 / 75 \mathrm{~nm}$, emission/band $\lambda 810 / 90 \mathrm{~nm}$ and dichromatic mirror at $\lambda 760 \mathrm{~nm})($ Chroma Technologies, Bellows Falls, VT). Fluorescence was captured and analyzed using CellSens (Olympus) software. OG and ICG fluorescence intensity increases were determined by sum intensity per unit of metastatic lesion area relative to non-tumor brain regions.

\section{Quantitative autoradiography}

Fluorescence imaging slides and ${ }^{14} \mathrm{C}$-AIB slides were placed in quantitative autoradiography (QAR) cassettes (FujiFilm Life Sciences, Stanford, CT) along with ${ }^{14} \mathrm{C}$ autoradiographic standards (American Radiochemicals, St. Louis, MO). A phosphor screen (FujiFilm Life Sciences, $20 \times 40$ super-resolution) was placed with the slides and standards and allowed to develop for 21 days. QAR phosphor screens were developed in a high-resolution phosphor-imager (GE Typhoon FLA 7000, Uppsala, Sweden) and converted to digital images, which were then calibrated to ${ }^{14} \mathrm{C}$ standards and analyzed using MCID Analysis software (InterFocus Imaging LTD, Linton, Cambridge, England). Metastases permeability fold-changes were calculated based on ${ }^{14} \mathrm{C}$-AIB signal intensity within confirmed metastases locations (determined using cresyl violet and Tomato Red fluorescence intensity overlays) relative to non-tumor brain ${ }^{14} \mathrm{C}$-AIB signal intensity.

\section{Tumor Staining}

Tissue sections were processed as described above. After allowing tissues to become adherent to charged slides overnight, slides were briefly dipped in PBS. Staining 
was performed using $0.1 \%$ cresyl violet acetate (Sigma-Aldrich, St. Louis, MO) (2 minutes) followed by briefly rinsing in tap water. Sections were cleared in $70 \%$ ethanol (15 seconds), 95\% ethanol (30 seconds), 100\% ethanol (30 seconds), respectively. Images were obtained with a 2x objective on the Olympus MVX microscope.

\section{Cytotoxicity assays}

Following the creation of PC-9-Br, in vitro MTT toxicity was performed to determine potential differences in drug sensitivity between the parenteral line and the brain-seeking line. 5,000 cells per well were plated in a 96-well plate and allowed to attach overnight in an incubator. The next day, after aspiration, drugs were added to wells. For all cytotoxicity assays, drugs were dissolved in DMSO or media to create a 10 $\mathrm{mM}$ stock, then further diluted in media to create standard concentrations. If drugs were dissolved in DMSO, similar amounts of DMSO were added to control wells. Plates were then incubated for 72 hours. $50 \mu \mathrm{L}$ of a $1 \mathrm{mg} / \mathrm{mL}$ solution of MTT was then added per well and incubated for another 2 hours. Wells were then aspirated and $100 \mu \mathrm{L}$ of DMSO was added per well and gently shaken for 1 hour to solubilize formazan crystals. Plates were then read at $570 \mathrm{nM}$ on the Synergy Hybrid 1 plate reader. Fluorescence values were converted to percentages based on the control average and plotted against a logscale of drug concentration.

\section{Statistics}

All statistics were performed on GraphPad Prism software. XY plots were analyzed by linear regression. Median and interquartile ranges are used for permeability changes and size of metastases. A D'Agostino and Pearson omnibus test was performed 
and determined a non-Gaussian distribution of data. Statistical analysis of permeability and size was performed using the non-parametric Kruskal-Wallis test followed by Dunn's multiple comparison test. Animal median survival data was analyzed using the log-rank test. Significance for all tests was defined as $\mathrm{p}<0.05$.

\subsection{Results}

The sixth round of PC-9 injections predominantly seeds the brain and has shorter survival than the parenteral line

Using the method developed by Yoneda et al, PC-9 cells were injected and extracted through six rounds in female nude mice. The cells from this sixth round are "brain-seeking" and the line is now termed PC-9-Br. Fig. 5.1 shows the distribution of the sixth round of PC9 injections $(\mathbf{A})$, stills from a 3D reconstruction of a mouse with brain tumor (B-E), and the survival curve of the parenteral and brain-seeking PC-9 line $(\mathbf{F})$. While the median survival was 61.5 days $(\mathrm{n}=2)$ in the parenteral line, the median survival for the brain-seeking line is significantly shorter at 45.5 days $(n=4)(p<0.05)$.

\section{Oregon Green is a substrate for P-glycoprotein efflux}

In order to determine if $\mathrm{OG}$ is a P-glycoprotein substrate, a Fluoroblock transwell experiment featuring increasing concentrations of $\mathrm{OG}$ and a Texas Red dextran $3 \mathrm{kDa}$ control were performed. A normalized time-lapse of OG (Fig. 5.2A) revealed increased concentrations when given the P-gp inhibitor tariquidar $1 \mu \mathrm{M}$, while a time-lapse of Texas Red dextran (Fig. 5.2A) showed no significant changes through time when exposed to tariquidar. For comparison, (Fig. 5.2B) raw fluorescence units show similar findings. A comparison of OG and ${ }^{14} \mathrm{C}-\mathrm{AIB}$ in the drug models (Fig. 5.2C-H) reveals 
similar results to the effects of rhodamine 123 and ${ }^{14} \mathrm{C}$-AIB in BCBM models, indicating that OG is effluxed by tumors due to P-glycoprotein. [8]

\section{PC-9-Br creates numerous, widespread, and various sized brain metastases}

To view the tumor and vascular distribution of $\mathrm{PC}-9-\mathrm{Br}$, an intracardiac perfusion of OG was performed on a vehicle-treated PC-9-Br mouse. After extraction, the brain was placed on a glass petri dish and imaged on the Olympus MVX. Fig. 5.3 presents the metastatic lesions and cerebral vasculature from the frontal cortex to the cerebellum. Bioluminescence (Fig. 5.3A) and fluorescence (Fig. 5.3B) outline the location of tumors within the brain. Four coronal slices were taken $800-1600 \mu \mathrm{m}$ apart, which are depicted in a brain atlas (Fig. 5.3 C1-F4).

\section{The PC-9-Br line forms spinal metastases due to ventricular infiltration}

While the majority of PC-9-Br tumor burden is within the brain, vertebral lesions often develop. This is an expected distribution of most brain-seeking tumor lines, with spinal cord affinity as a theorized mechanism. [9] Fig. 5.4 shows a brain slice of a vehicle mouse with a tumor on the periphery of the right lateral ventricle, with the choroid plexus separating the tumor into two sections. On bioluminescence imaging, a spinal lesion is noted in the mouse that this brain was taken from (Fig. 5.4A). Within the ventricle, tumor cells are seen co-opting the choroid plexus, which contains OG (Fig. 5.4B-D).

PC-9-Br are less sensitive to conventional and targeted lung chemotherapy than parenteral PC-9 in-vitro

A cytotoxic assay was performed to evaluate the chemotherapeutic sensitivity of the parenteral and brain-seeking PC9 lines. In the parenteral line, SN-38 sensitivity is 7 
$\mathrm{nM}$, more than two orders of magnitude lower than the $6 \mu \mathrm{M} \mathrm{SN}-38$ sensitivity of the brain-seeking line. The etoposide sensitivity of the parenteral line $(408 \mathrm{nM})$ is more than 100-fold lower than in PC-9-Br $(6.4 \mu \mathrm{M})$. Pemetrexed sensitivity is modestly higher in the PC-9-Br (512 nM) compared to PC-9-P (391 nM), while cisplatin sensitivity is slightly lower (593 nM vs. $669 \mathrm{nM}$, respectively). This trend is seen again in targeted tyrosine kinase inhibitors. While the parenteral line is sensitive in the femtomolar range to erlotinib, gefitinib, afatinib, and osimertinib, the PC-9-Br sensitivities are in the nanomolar range, more than a 10 -fold difference. A representative $\mathrm{IC}_{50}$ curve demonstrating the reduced sensitivity of PC-9-Br to PC-9-P is depicted in Fig. 5.5. The PC-9-Br are more sensitive to tyrosine kinase inhibitors than conventional lung chemotherapy agents and SN-38 (Table 5.1).

\section{Vehicle treated tumors are significantly more permeable to ${ }^{14} \mathrm{C}$-AIB and have}

\section{higher OG but not ICG fluorescence intensity than non-tumor regions}

As animals became moribund with neurological symptoms, we sought to determine the extent and differences of passive permeability, P-gp efflux, and vascularity of control and drug-treated tumors via use of three different molecular weight markers, starting with vehicle (Fig. 5.6). Passive permeability changes in vehicle metastatic lesions ranged from 0.4505 to 38.39 -fold over normal brain with a median (IQR) fold change of $3.254(1.928-5.971)$ for ${ }^{14} \mathrm{C}-\mathrm{AIB}$ (Fig. 5.6F), which were significantly higher than nontumor regions $(\mathrm{p}<0.01)$. For OG, fluorescence intensity ranged from 0.9974 to 1.271 fold with a median (IQR) fold change of 1.007 (1.004-1.013), which was significantly higher than non-tumor regions $(\mathrm{p}<0.01)$. For ICG, fluorescence intensity ranged from 0.9866 to 1.053 -fold with a median (IQR) fold change of $1.0(0.9953-1.002)$, which was 
not significantly higher than non-tumor regions $(\mathrm{p}>0.05)$. No correlation was observed $\left(\mathrm{r}^{2}<0.02\right)$ for $\mathrm{OG}$, ICG, or ${ }^{14} \mathrm{C}$-AIB passive permeability and metastasis size (Fig. 5.6G).

Cisplatin-etoposide treated tumors are significantly more permeable to ${ }^{14} \mathrm{C}$ $A I B$, and have higher $O G$ and ICG fluorescence intensity than non-tumor regions

After seeing a positive trend in vehicle tumors, we turned our sights on characterizing tumors treated with conventional lung therapy, starting with the cisplatin doublet therapy with etoposide (Fig. 5.7). Passive permeability changes in cisplatinetoposide metastatic lesions ranged from 0.3021 to 18.55 -fold over normal brain with a median (IQR) fold change of $1.231(0.8541-2.077)$ for ${ }^{14}$ C-AIB (Fig. 5.7F), which was significantly higher than non-tumor regions $(\mathrm{p}<0.01)$. For OG, fluorescence intensity ranged from 0.9891 to 1.190 -fold with a median (IQR) fold change of 1.020 (1.007 1.037), which was significantly higher than non-tumor regions $(\mathrm{p}<0.01)$. For ICG, fluorescence intensity ranged from 0.9597 to 1.078 -fold with a median (IQR) fold change of $0.9885(0.9810-1.001)$, which was significantly higher than non-tumor regions $(\mathrm{p}>$ 0.01). There was a no correlation $\left(\mathrm{r}^{2}=0.07\right)$ to changes in ${ }^{14} \mathrm{C}$-AIB permeability and lesion size, while a moderate correlation was observed $\left(r^{2}=0.42\right)$ for OG but not ICG $\left(r^{2}\right.$ $=0.03)$ fluorescence intensity and metastasis size in the cisplatin-etoposide model (Fig. 5.7G).

Cisplatin-pemetrexed treated tumors are significantly more permeable to ${ }^{14} \mathrm{C}$ $A I B$ and have higher $O G$ but not ICG fluorescence intensity than non-tumor regions

After seeing a positive trend in the cisplatin-etoposide doublet therapy, we turned our sights on characterizing the cisplatin-pemetrexed doublet therapy (Fig. 5.8). Passive 
permeability changes in cisplatin-pemetrexed brain tumors ranged from 0.1601 to 24.83 -

fold over normal brain with a median (IQR) fold change of $4.235(1.681-7.046)$ for ${ }^{14} \mathrm{C}$ AIB (Fig. 5.8F), which was significantly higher than non-tumor regions $(\mathrm{p}<0.01)$. For OG, fluorescence intensity ranged from 0.0652 to 1.565 -fold with a median (IQR) fold change of $1.049(1.010-1.144)$, which was significantly higher than non-tumor regions $(\mathrm{p}<0.01)$. For ICG, fluorescence intensity ranged from 0.5926 to 4.490 -fold with a median (IQR) fold change of 0.9991 (0.9941 - 1.005), which was not significantly higher than non-tumor regions $(p>0.05)$. There was a moderate correlation $\left(r^{2}=0.44\right)$ in ${ }^{14} \mathrm{C}$ AIB permeability and lesion size. No correlation was observed for OG $\left(r^{2}=0.12\right)$ or ICG $\left(r^{2}=0.03\right)$ fluorescence intensity and metastasis size in the cisplatin-pemetrexed model (Fig. 5.8G).

Gefitinib treated tumors are significantly more permeable to ${ }^{14} \mathrm{C}$-AIB and have higher OG but not ICG fluorescence intensity than non-tumor regions

Aside from platinum doublet therapy, EGFR-mutated tumors can be treated with targeted tyrosine kinase inhibitors. Gefitinib represents a first-generation targeted EGFRinhibitor (Fig. 5.9). Passive permeability changes in gefitinib brain tumors ranged from 0.7222 to 12.94 -fold over normal brain with a median (IQR) fold change of 4.537 (2.707 - 8.20) for ${ }^{14}$ C-AIB (Fig. 5.9F), which was significantly higher than non-tumor regions $(\mathrm{p}<0.01)$. For OG, fluorescence intensity ranged from 0.9747 to 1.352 -fold with a median (IQR) fold change of 1.028 (1.006 - 1.066), which was significantly higher than non-tumor regions $(\mathrm{p}<0.01)$. For ICG, fluorescence intensity ranged from 0.9985 to 1.070-fold with a median (IQR) fold change of $1.030(1.002-1.042)$, which was not significantly higher than non-tumor regions $(\mathrm{p}>0.05)$. There was a moderate correlation 
$\left(r^{2}=0.33\right)$ to changes in ${ }^{14} \mathrm{C}$-AIB permeability and lesion size. No correlation was observed for OG or ICG $\left(\mathrm{r}^{2}<0.03\right)$ fluorescence intensity and metastasis size in the gefitinib model (Fig. 5.9G).

Afatinib treated tumors are significantly more permeable to ${ }^{14} \mathrm{C}$-AIB, and have higher OG and ICG fluorescence intensity than non-tumor regions

Afatinib represents a second-generation EGFR-tyrosine kinase inhibitor (Fig. 5.10). Passive permeability changes in afatinib brain tumors ranged from 0.2958 to 7.936-fold over normal brain with a median (IQR) fold change of $1.315(0.8746-2.197)$ for ${ }^{14} \mathrm{C}$-AIB (Fig. 5.10F), which was significantly higher than non-tumor regions $(\mathrm{p}<$ 0.01). For OG, fluorescence intensity ranged from 0.9854 to 1.080 -fold with a median (IQR) fold change of $1.011(1.003-1.021)$, which was significantly higher than nontumor regions $(\mathrm{p}<0.01)$. For ICG, fluorescence intensity ranged from 0.9975 to 1.055 fold with a median (IQR) fold change of 1.001 (0.9957 - 1.007), which was also significantly higher than non-tumor regions $(\mathrm{p}<0.05)$. There was a no correlation $\left(\mathrm{r}^{2}<\right.$ 0.01 ) to changes in ${ }^{14} \mathrm{C}$-AIB, OG, or ICG passive permeability and metastasis size in the afatinib model (Fig. 5.10G).

Osimertinib treated tumors are significantly more permeable to ${ }^{14} \mathrm{C}-\mathrm{AIB}$, and have higher OG and ICG fluorescence intensity non-tumor regions

Osimertinib represents a third-generation EGFR-tyrosine kinase inhibitor (Fig. 5.11). Passive permeability changes in osimertinib brain tumors ranged from 0.1618 to 8.891-fold over normal brain with a median (IQR) fold change of $1.950(1.328-3.033)$ for ${ }^{14} \mathrm{C}$-AIB (Fig. 5.11F), which was significantly higher than non-tumor regions ( $\mathrm{p}<$ 
0.01). For OG, fluorescence intensity ranged from 0.9703 to 1.375 -fold with a median (IQR) fold change of 1.077 (1.041 - 1.138), which was significantly higher than nontumor regions $(\mathrm{p}<0.01)$. For ICG, fluorescence intensity ranged from 0.9862 to $1.054-$ fold with a median (IQR) fold change of 1.006 (1.002 - 1.020), which was also significantly higher than non-tumor regions $(\mathrm{p}<0.01)$. There was a no correlation $\left(\mathrm{r}^{2}<\right.$ 0.05 ) to changes in ${ }^{14} \mathrm{C}-\mathrm{AIB}$, OG, or ICG passive permeability and metastasis size in the osimertinib model (Fig. 5.11G).

\section{${ }^{14} \mathrm{C}$-AIB permeability is associated with chemotherapeutic efficacy}

A comparison of fold increases of OG and ICG fluorescence increases across drug treated groups reveals that most tumor regions are similar to control areas (Fig. 5.12). In comparison, ${ }^{14} \mathrm{C}$-AIB passive permeability in tumors is much higher, ranging from 3 - to 12-fold above non-tumor regions. Lower ${ }^{14} \mathrm{C}$-AIB fold increases over control areas are found in cisplatin-etoposide, afatinib, and osimertinib-treated groups, which are the only treatment groups to increase survival.

\section{PC-9-Br tumor size and number are moderately correlated with survival}

After survival endpoints were reached, brains were sliced and analyzed for tumor count. A D'Agostino \& Pearson omnibus normality test showed no compounds tested displayed a Gaussian distribution, so median and interquartile range column statistics were calculated (Table 5.2). Of 114 vehicle tumors, median tumor size was $0.1844 \mathrm{~mm}^{2}$ (IQR: 0.1129 - 0.3097) with a 42-day median survival. Of 117 cisplatin-etoposide tumors, median tumor size was $0.1093 \mathrm{~mm}^{2}$ (IQR: $0.0533-0.2384$ ) with a 51.5 -day median survival. Of 110 cisplatin-pemetrexed tumors, median tumor size was 0.2492 
$\mathrm{mm}^{2}$ (IQR: $0.1305-0.4054$, with a 45-day median day survival. Of 34 gefitinib tumors, median tumor size was $0.5530 \mathrm{~mm}^{2}$ (IQR: $0.2868-0.6787$ ), with a 48-day median survival. Of 85 afatinib tumors, median tumor size was $0.1459 \mathrm{~mm}^{2}$ (IQR: $0.0687-$ 0.2809) with a 73-day median survival. Of 56 osimertinib tumors, median tumor size was $0.1293 \mathrm{~mm}^{2}$ (IQR: $0.0629-0.2125$ ), with a 73-day median survival. Linear regression of median metastases size and days of survival on chemotherapy produced an $r^{2}$ of 0.3461 , while a linear regression of tumor number and survival produced an $r^{2}$ of 0.22 .

\section{Distribution of PC-9-Br tumors follow surface area and blood flow patterns}

After slicing and characterizing dye fold increases, we sought to determine the distribution of tumors within the brain (Fig. 5.13). The majority of tumors occur in the cortex regardless of treatment (Fig. 5.13J). Blood flow is approximately $1 \mathrm{~mL} / \mathrm{g} / \mathrm{min}$ in these mouse regions, and so the plot of tumors based on brain blood flow (Fig. 5.13K) resembles that of total number of tumors (Fig. 5.13J). Gefitinib was observed to have the lowest number of tumors of the brains analyzed.

\subsection{Discussion}

As tumors grow, their requirement for enhanced nutrition stimulates the formation of new vasculature that joins nearby blood vessels in a process known as angiogenesis. [10] As vessels form, they are initially immature and compromised; the resultant loss of barrier integrity allows passive permeation of formerly restricted substances into tumor and brain parenchyma. [11]

Typical chemotherapy sizes range from a molecular weight of 300 (nucleotide analogues) to more than 100,000 (antibodies). Cisplatin (300 Da), etoposide (589 Da), 
pemetrexed (472 Da), gefitinib (447 Da), afatinib (486 Da), and osimertinib (500 Da) are clinically used therapeutics in the treatment of lung cancer. The size range of these agents are encompassed by ${ }^{14} \mathrm{C}$-AIB (103 Da) and the P-gp substrate OG (509 Da), with albumin-bound ICG representing antibody size and vascularity. [12, 13]

To create a brain metastatic model, two main methods exist: intracardiac and intracarotid injections. [14] While intracarotid injections deliver cancer cells directly to the brain compared to intracardiac injections which allow cancer cells to circulate throughout the arterial system, intracarotid injections are much more invasive and timeconsuming, and often have similar results to intracardiac injections. [15] Our method of injection occasionally leads to mandibular metastases (S1) which are often found in preclinical brain metastases models as well as clinical settings. [16-18] Of note, ventricular infiltration and development of spinal metastases have been noted in BCBM models as well. [19, 20]

The PC-9 cell line is commonly utilized in preclinical lung cancer research to evaluate the effects of chemotherapy in an EGFR-mutant model. [21-23] PC-9 are sensitive to first generation (gefitinib) and second generation (afatinib) tyrosine kinase inhibitors, and can be induced to form the T790M mutation which often leads to drug resistance and relapse in the clinical setting. [24, 25] Osimertinib, a third generation inhibitor, overcomes the T790M mutation and is used when resistance to first or second generation inhibitors forms. Non-T790M mutated PC-9 cells are also sensitive to osimertinib, which is why it was included in our trial. [21, 26]

Similar to preclinical breast cancer brain metastases (BCBM), PC-9-Br tumors are generally less than $1 \mathrm{~mm}^{2}$, however PC-9-Br are far less permeable to both ${ }^{14} \mathrm{C}$-AIB and 
similarly-sized fluorescent markers. [27] The median permeability of ${ }^{14} \mathrm{C}$-AIB in LCBM ranges between 1.2- to 4.5 -fold above control regions, while in BCBM the median permeability ranges between 2.1 to 12-fold above control regions. [27] Both BCBM and LCBM contrast with primary glioblastoma, whose lesions are much larger and much more permeable to ${ }^{14} \mathrm{C}$-AIB, with rates of transfer that near water diffusion. [28]

The effect of tumor size and survival is not yet fully understood. A preclinical model of BCBM showed that larger tumor sizes were associated with higher median survival. [27] In contrast, smaller median tumor sizes $\left(\leq 1.5 \mathrm{~mm}^{2}\right)$ were associated with longer median survival in our LCBM model. These smaller median tumor sizes were also linked with therapy that extended median survival, thus portraying a therapy-size-survival interaction. Our LCBM findings correspond with targeted inhibitors showing reduction of brain metastases size in the clinical setting. [29, 30]

Much like the BCBM models, the PC-9-Br line expresses the efflux pump Pglycoprotein. [8] In this model, rhodamine 123 (Rh123) was perfused and compared against ${ }^{14} \mathrm{C}$-AIB fold changes in tumor regions and control areas. Much like our OG results, Rh123 values ranged between 0.9 and 1.5 -fold above non-tumor regions, indicating that P-gp exists at the tumor barrier and though some dye does passively permeate into tumor, OG is also a substrate for P-gp-mediated efflux.

\subsection{Conclusion}

The EGFR-mutant PC-9-Br creates many scattered brain metastases, most of which are $\leq 1.0 \mathrm{~mm}^{2}$. These tumors are similarly permeable to breast cancer brain metastases and have active P-glycoprotein to efflux chemotherapy. Conventional chemotherapy such as cisplatin, etoposide, and pemetrexed are not as effective in 
increasing median survival as targeted EGFR inhibitors, especially second (afatinib) and third (osimertinib) generation agents. Tumors treated with these agents have smaller tumor sizes and lower ${ }^{14} \mathrm{C}$-AIB permeability.

\subsection{References}

1. Wong, M.C.S., et al., Incidence and mortality of lung cancer: global trends and association with socioeconomic status. Sci Rep, 2017. 7(1): p. 14300.

2. Chi, A. and R. Komaki, Treatment of brain metastasis from lung cancer. Cancers (Basel), 2010. 2(4): p. 2100-37.

3. Shojaee, S. and P. Nana-Sinkam, Recent advances in the management of nonsmall cell lung cancer. F1000Res, 2017. 6: p. 2110.

4. Lockman, P.R., et al., Heterogeneous blood-tumor barrier permeability determines drug efficacy in experimental brain metastases of breast cancer. Clin Cancer Res, 2010. 16(23): p. 5664-78.

5. Murrell, D.H., et al., Understanding Heterogeneity and Permeability of Brain Metastases in Murine Models of HER2-Positive Breast Cancer Through Magnetic Resonance Imaging: Implications for Detection and Therapy. Transl Oncol, 2015. 8(3): p. 176-84.

6. Percy, D.B., et al., In vivo characterization of changing blood-tumor barrier permeability in a mouse model of breast cancer metastasis: a complementary magnetic resonance imaging approach. Invest Radiol, 2011. 46(11): p. 718-25.

7. Yoneda, T., et al., A bone-seeking clone exhibits different biological properties from the MDA-MB-231 parental human breast cancer cells and a brain-seeking clone in vivo and in vitro. J Bone Miner Res, 2001. 16(8): p. 1486-95. 
8. Adkins, C.E., et al., P-glycoprotein mediated efflux limits substrate and drug uptake in a preclinical brain metastases of breast cancer model. Front Pharmacol, 2013. 4: p. 136.

9. Nguyen, D.X., et al., WNT/TCF signaling through LEF1 and HOXB9 mediates lung adenocarcinoma metastasis. Cell, 2009. 138(1): p. 51-62.

10. Weis, S.M. and D.A. Cheresh, Tumor angiogenesis: molecular pathways and therapeutic targets. Nat Med, 2011. 17(11): p. 1359-70.

11. Bohn, K.A., et al., Inhibition of VEGF and Angiopoietin-2 to Reduce Brain Metastases of Breast Cancer Burden. Front Pharmacol, 2017. 8: p. 193.

12. Yoneya, S., et al., Binding properties of indocyanine green in human blood. Invest Ophthalmol Vis Sci, 1998. 39(7): p. 1286-90.

13. Alander, J.T., et al., A review of indocyanine green fluorescent imaging in surgery. Int J Biomed Imaging, 2012. 2012: p. 940585.

14. Saxena, M. and G. Christofori, Rebuilding cancer metastasis in the mouse. Mol Oncol, 2013. 7(2): p. 283-96.

15. Balathasan, L., J.S. Beech, and R.J. Muschel, Ultrasonography-guided intracardiac injection: an improvement for quantitative brain colonization assays. Am J Pathol, 2013. 183(1): p. 26-34.

16. Budde, M.D., et al., Differential microstructure and physiology of brain and bone metastases in a rat breast cancer model by diffusion and dynamic contrast enhanced MRI. Clin Exp Metastasis, 2012. 29(1): p. 51-62.

17. Rajinikanth, M., et al., Metastasis of lung adenocarcinoma to the jaw bone. J Oral Maxillofac Pathol, 2015. 19(3): p. 385-8. 
18. Dirican, N., et al., Mandible bone metastases secondary to pulmonary adenocarcinoma. Med Arch, 2014. 68(5): p. 356-8.

19. Fitzgerald, D.P., et al., Reactive glia are recruited by highly proliferative brain metastases of breast cancer and promote tumor cell colonization. Clin Exp Metastasis, 2008. 25(7): p. 799-810.

20. Samala, R., et al., Vinorelbine Delivery and Efficacy in the MDA-MB-231BR Preclinical Model of Brain Metastases of Breast Cancer. Pharm Res, 2016. 33(12): p. 2904-2919.

21. Park, M.Y., et al., Generation of lung cancer cell lines harboring EGFR T790M mutation by CRISPR/Cas9-mediated genome editing. Oncotarget, 2017. 8(22): p. 36331-36338.

22. Hamamoto, J., et al., Non-small cell lung cancer PC-9 cells exhibit increased sensitivity to gemcitabine and vinorelbine upon acquiring resistance to EGFRtyrosine kinase inhibitors. Oncol Lett, 2017. 14(3): p. 3559-3565.

23. Koizumi, F., et al., Establishment of a human non-small cell lung cancer cell line resistant to gefitinib. Int J Cancer, 2005. 116(1): p. 36-44.

24. Zou, B., et al., Deciphering mechanisms of acquired T790M mutation after EGFR inhibitors for NSCLC by computational simulations. Sci Rep, 2017. 7(1): p. 6595.

25. Wang, S., S. Cang, and D. Liu, Third-generation inhibitors targeting EGFR T790M mutation in advanced non-small cell lung cancer. J Hematol Oncol, 2016. 9: p. 34 . 
26. Ballard, P., et al., Preclinical Comparison of Osimertinib with Other EGFR-TKIs in EGFR-Mutant NSCLC Brain Metastases Models, and Early Evidence of Clinical Brain Metastases Activity. Clin Cancer Res, 2016. 22(20): p. 5130-5140.

27. Adkins, C.E., et al., Characterization of passive permeability at the blood-tumor barrier in five preclinical models of brain metastases of breast cancer. Clin Exp Metastasis, 2016. 33(4): p. 373-83.

28. Mittapalli, R.K., et al., Quantitative Fluorescence Microscopy Measures Vascular Pore Size in Primary and Metastatic Brain Tumors. Cancer Res, 2017. 77(2): p. 238-246.

29. Koba, T., et al., Rapid intracranial response to osimertinib, without radiotherapy, in nonsmall cell lung cancer patients harboring the EGFR T790M mutation: Two Case Reports. Medicine (Baltimore), 2017. 96(6): p. e6087.

30. Watanabe, S., H. Hayashi, and K. Nakagawa, Is afatinib a treatment option for brain metastases in patients with EGFR mutation-positive non-small cell lung cancer? Ann Transl Med, 2016. 4(11): p. 225. 

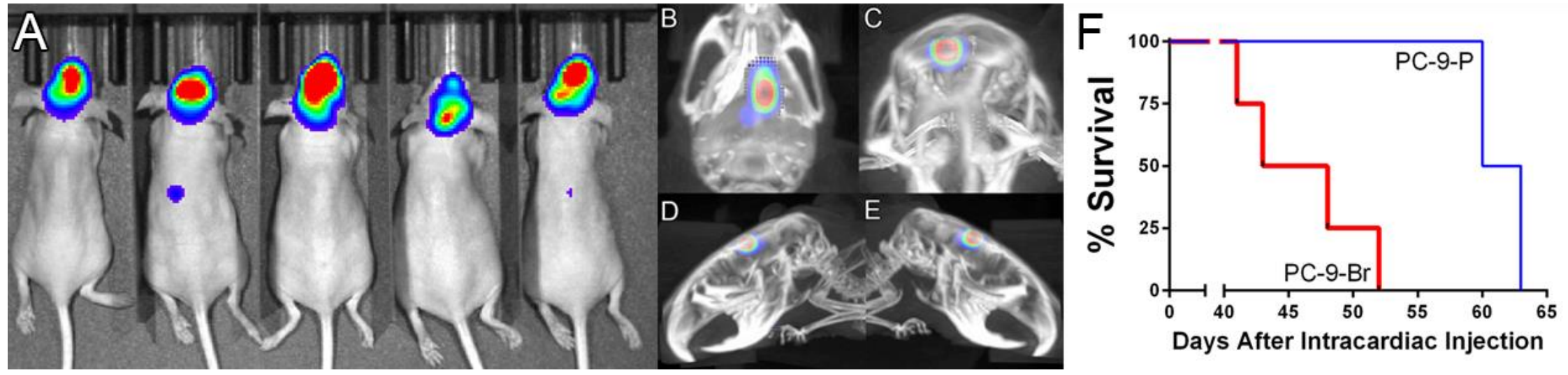

\section{Figure 5.1: Distribution and survival of the brain-seeking PC-9 cell line.}

(A) Visualization of tumor burden in athymic nude female mice injected with PC-9-Br cells. The majority of tumor burden is within the brain, with a smaller amount of vertebral metastases. (B-E) Micro-CT reconstruction of a mouse with a PC-9-Br tumor shows the anatomical location of the tumor. (F) Median survival for the PC-9-P parenteral line is 61.5 days $(\mathrm{n}=2$ ), which is significantly reduced in brain-seeking PC-9-Br line $(45.5$ days, $\mathrm{n}=4)(\mathrm{p}<0.05)$. 

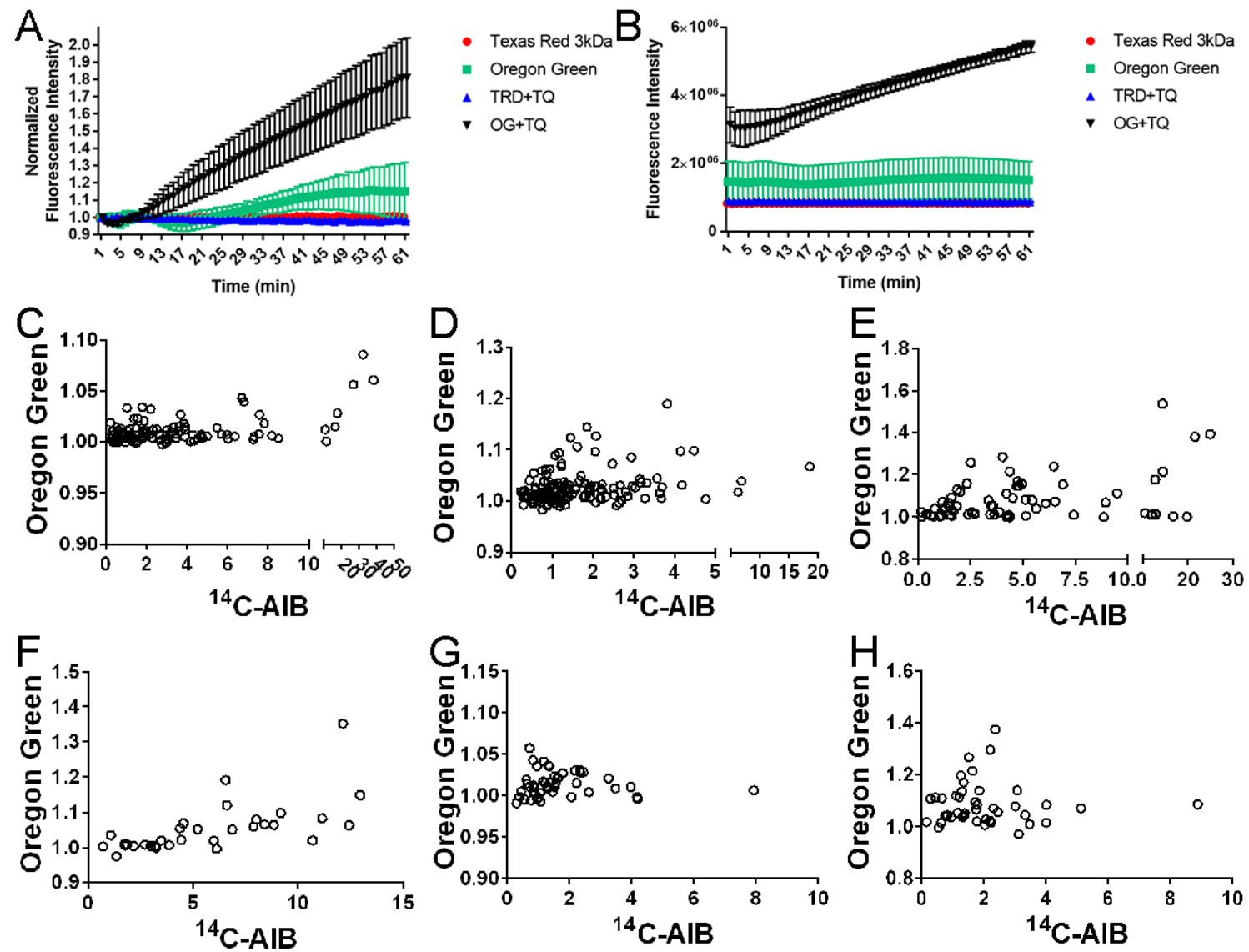

Figure 5.2. Oregon Green is a P-glycoprotein substrate.

(A) A Fluoroblock transwell experiment of Oregon Green \pm tariquidar $1 \mu \mathrm{M}$ and known non-P-gp substrate Texas Red Dextran 3kDa \pm TQ shows OG fluorescence increases over time when exposed to TQ, with data normalized for ease of comparison. (B) Unadjusted data is shown for comparative purposes. (C) Vehicle, (D) $\mathrm{C}+\mathrm{E},(\mathbf{E}) \mathrm{C}+\mathrm{P},(\mathbf{F})$ Gefitinib, (G) Afatinib, and (H) Osimertinib feature similar OG permeability ratios to known P-gp substrate rhodamine-123, signifying that $\mathrm{OG}$ is a P-gp substrate. All data is mean $\pm \mathrm{SEM}$. 


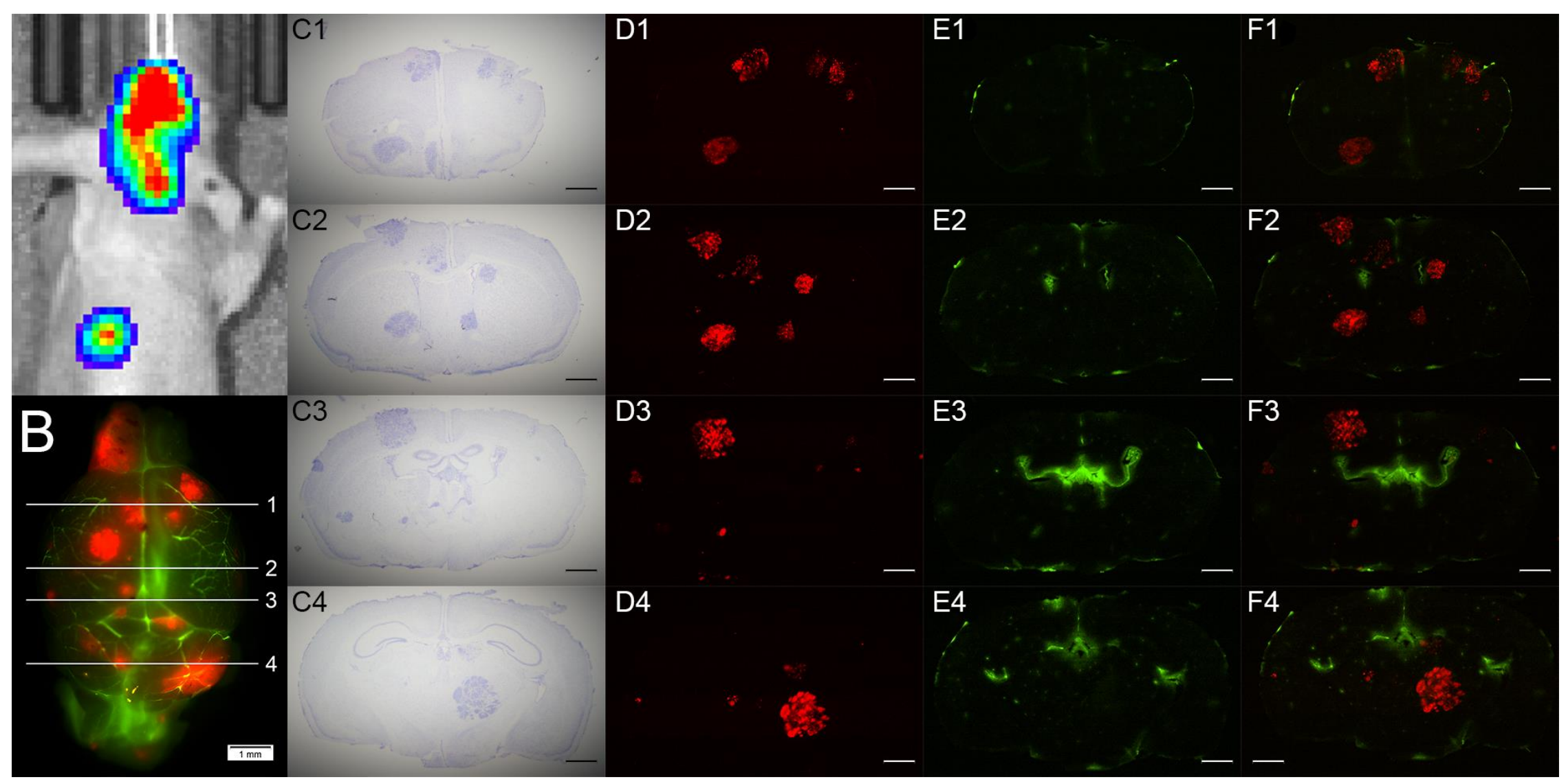

Figure 5.3. Distribution of brain metastases in PC-9-Br.

(A) Luciferin bioluminescence shows the large PC-9-Br tumor burden. (B) Fluorescence imaging contrasts the Oregon Greenperfused vasculature and the distribution of Tomato Red-expressing tumors. Four numbered slices $(1=\# \mathrm{~mm} ; 2=\# \mathrm{~mm} ; 3=\#$ $\mathrm{mm} ; 4=\# \mathrm{~mm}$ ) correspond to the coronal sections (C-F). (C) Visualization of brain metastases based on cresyl violet staining. (D) Tomato Red tumors accurately represent tumor burden confirmed by cresyl violet staining. (E) Oregon Green highlights normal and disrupted vasculature in tumor brain. (F) An overlay of Oregon Green and Tomato Red depicts tumor environment and vascular integrity. Scale bars $=1 \mathrm{~mm}$. 


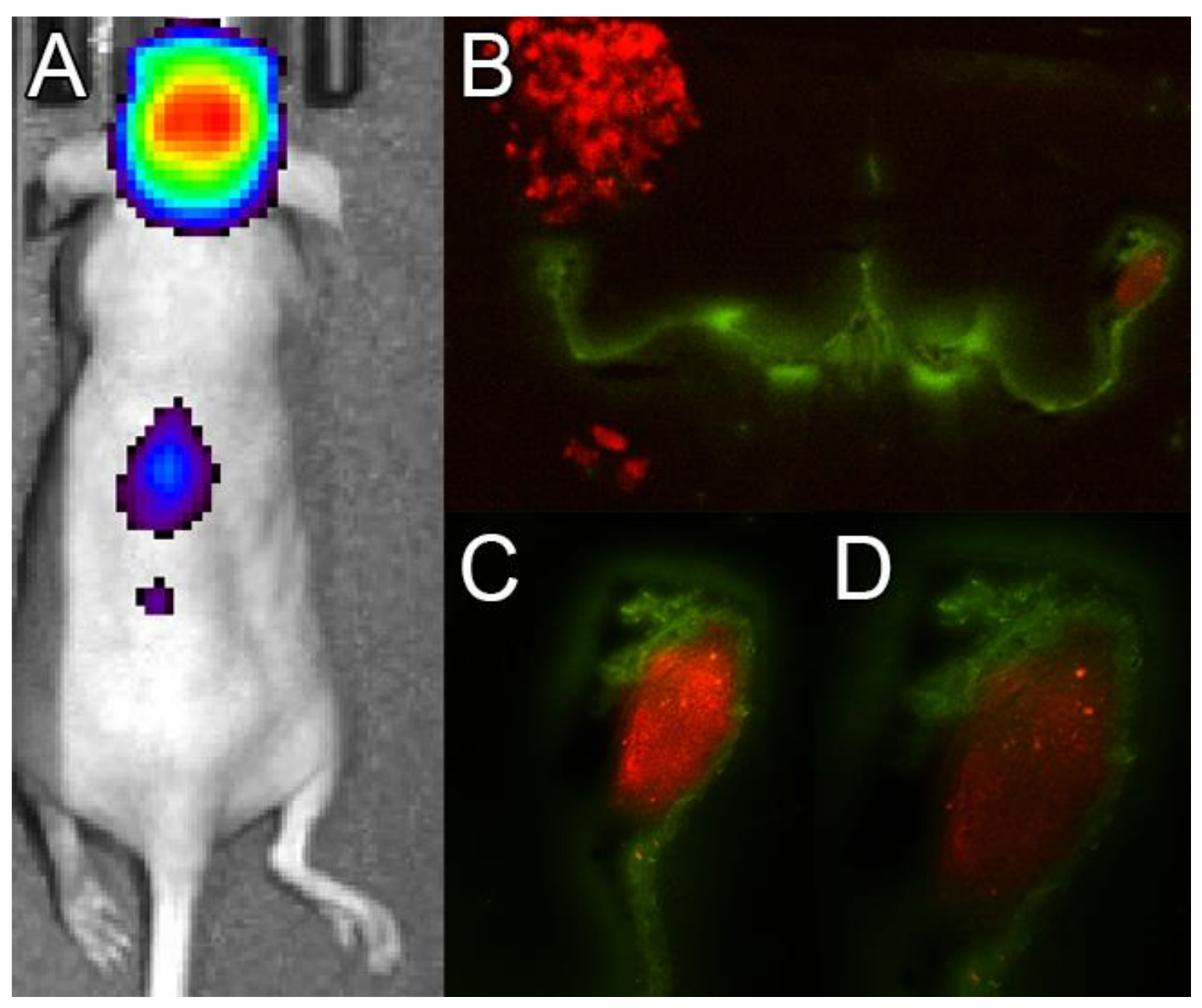

Figure 5.4. Ventricular infiltration leads to spinal tumor burden.

(A) Bioluminescence data shows a large tumor burden in the brain with smaller spinal lesions. The brain was extracted, sliced, and imaged. (B) Fluorescence imaging shows a Tomato Red tumor invading the lateral ventricle. (C and D) A tumor mass and individual cells are visible within the ventricle and around the choroid plexus (Oregon Green). 


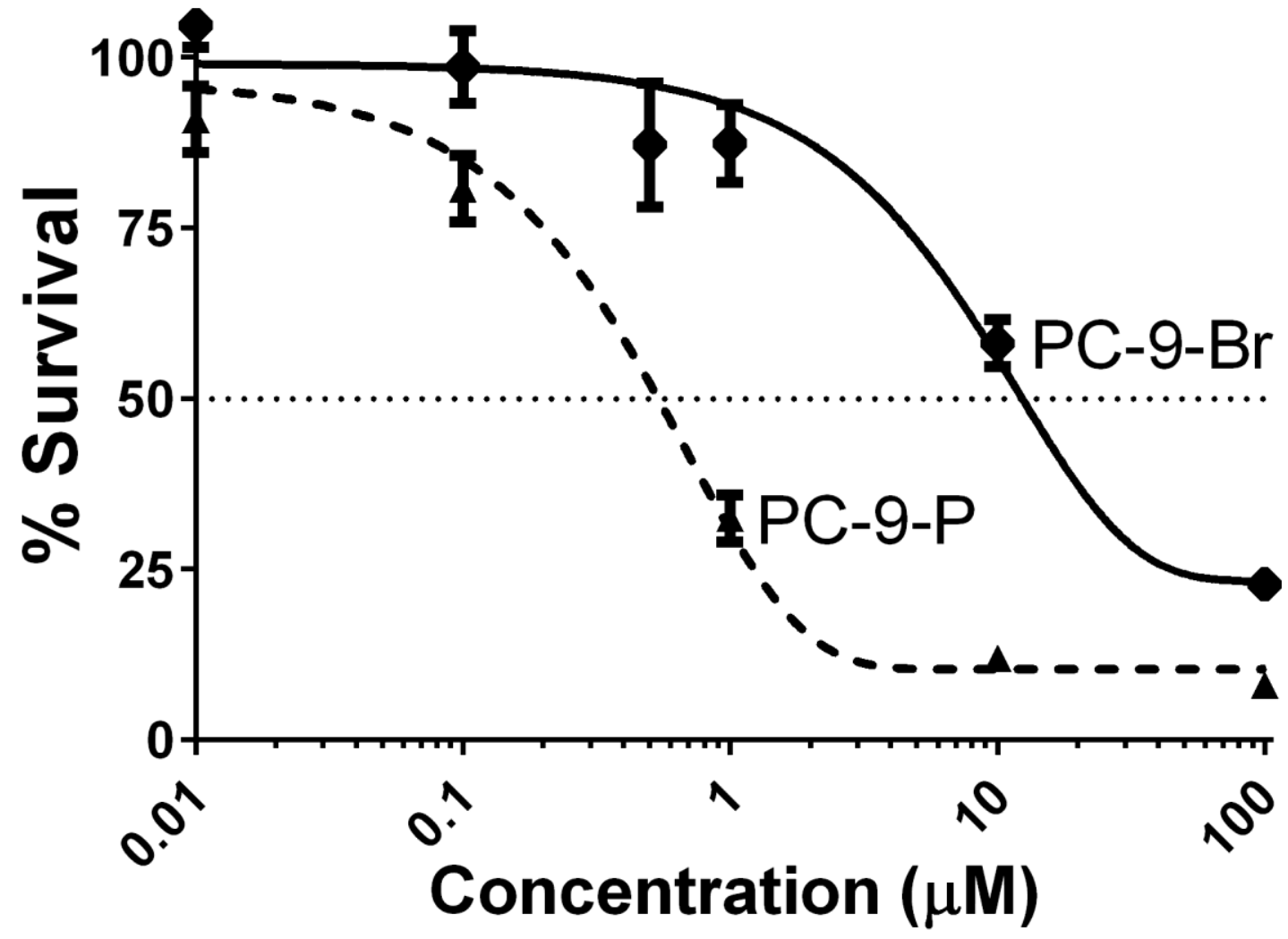

Figure 5.5. PC-9-Br is less sensitive to chemotherapy compared to the parenteral line.

A representative $\mathrm{IC}_{50}$ curve depicts the reduced etoposide sensitivity of PC-9-Br $(6.4 \mu \mathrm{M})$ compared to the PC-9-P line $(0.41 \mu \mathrm{M})$. 


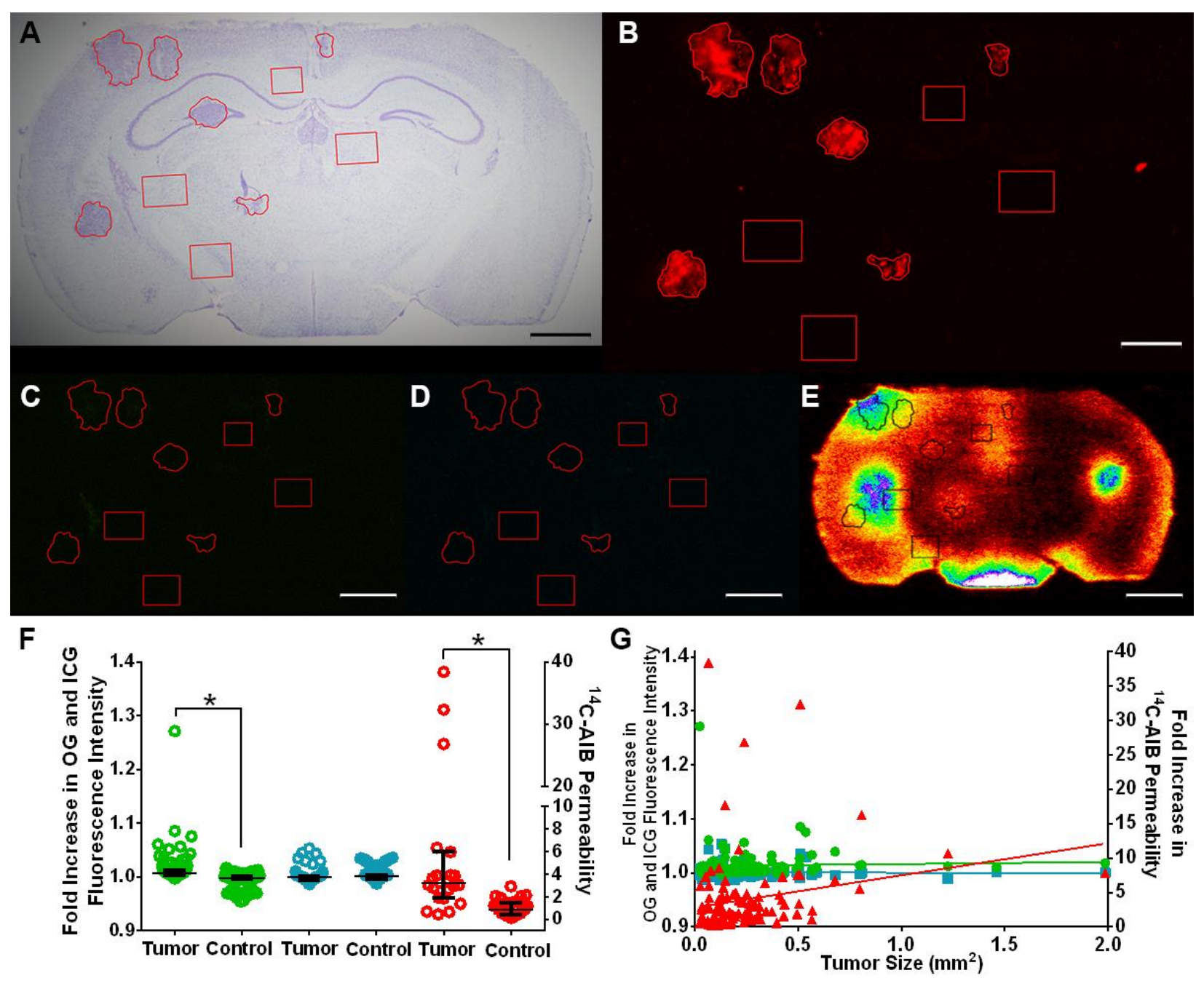

Figure 5.6. Permeability changes of PC-9-Br treated with vehicle.

(A) A representative cresyl violet brain slice (approximately corresponding to Fig. 3.3

C4) of vehicle-treated PC-9-Br tumors, with (B) corresponding Tomato Red tumor

fluorescence. (C) The same slice with Oregon Green, (D) ICG, and (E) ${ }^{14} \mathrm{C}-\mathrm{AIB}$ autoradiographic data to quantify permeability increases. (F) The median and interquartile ranges for fold-increases of passive permeability markers in 114 tumors over control regions. For vehicle brains, tumors were significantly more permeable to $\mathrm{OG}$ and ${ }^{14} \mathrm{C}$ AIB $(p<0.05)$, but not ICG $(p>0.05)$. (G) The fold increases of OG, ICG, or ${ }^{14} \mathrm{C}-\mathrm{AIB}$ were not correlated with metastases size $\left(\mathrm{r}^{2}<0.02\right)$. For all depicted brain slices, tumor regions are outlined while control areas are squares. Scale bar $=1 \mathrm{~mm}$. 

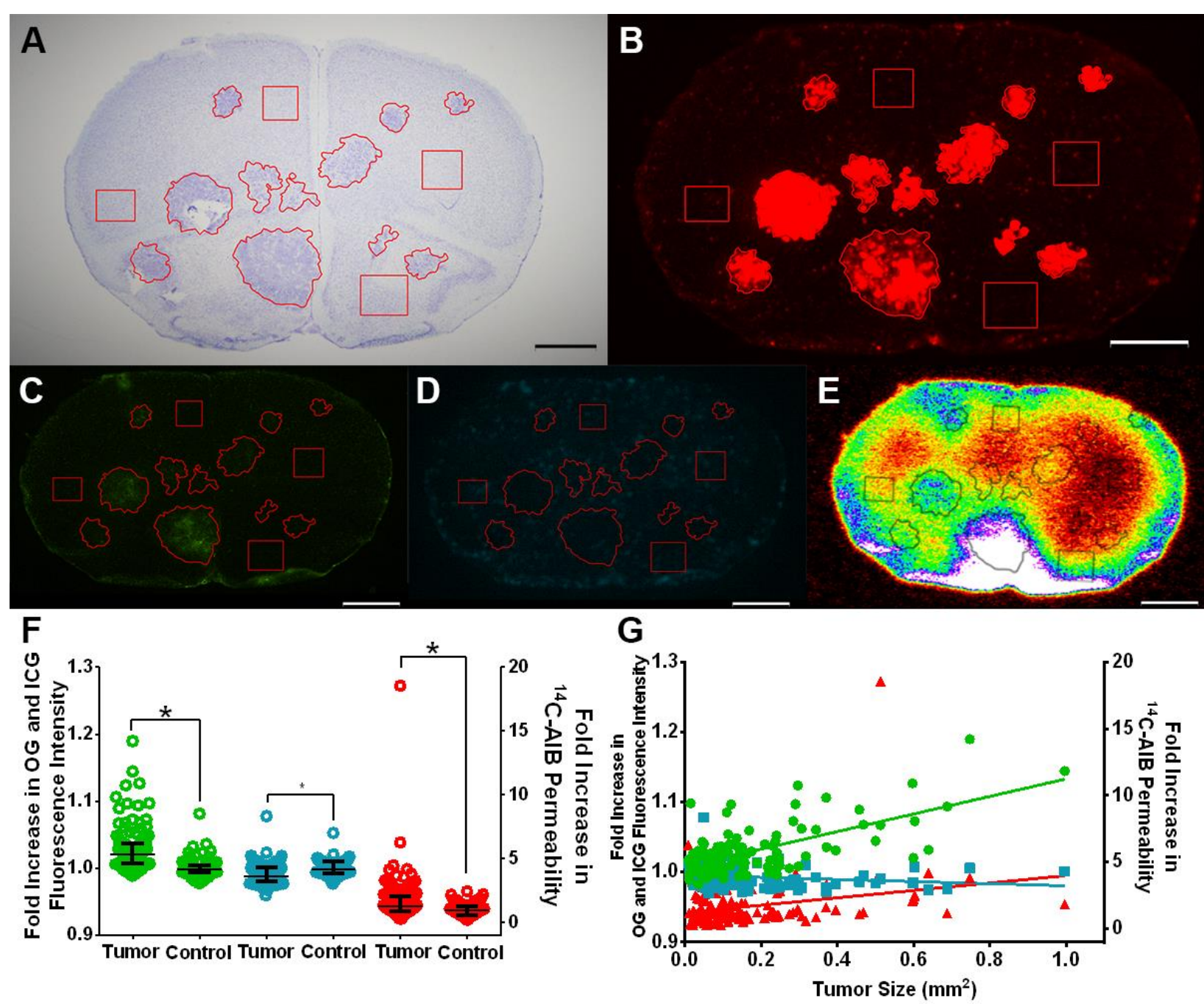

Figure 5.7. Passive permeability changes of PC-9-Br treated with cisplatinetoposide.

(A) A representative cresyl violet brain slice (approximately corresponding to Fig. 5.3

C1) of cisplatin-etoposide-treated PC-9-Br tumors, with (B) corresponding Tomato Red tumor fluorescence. (C) The same slice with Oregon Green, (D) ICG, and (E) ${ }^{14} \mathrm{C}$-AIB autoradiographic data to quantify P-gp, vascularity, and permeability increases, respectively. (F) The median and interquartile ranges for fold-increases of dyes in 117 tumors over control regions. For cisplatin-etoposide-treated brains, tumors were significantly more permeable ${ }^{14} \mathrm{C}$-AIB and had more P-gp and vascularity than control regions $(\mathrm{p}<0.05)$. (G) The fold increases of OG, ICG, or ${ }^{14} \mathrm{C}$-AIB were not correlated with metastases size $\left(r^{2}<0.02\right)$. For all depicted brain slices, tumor regions are outlined while control areas are squares. Scale bar $=1 \mathrm{~mm}$. 

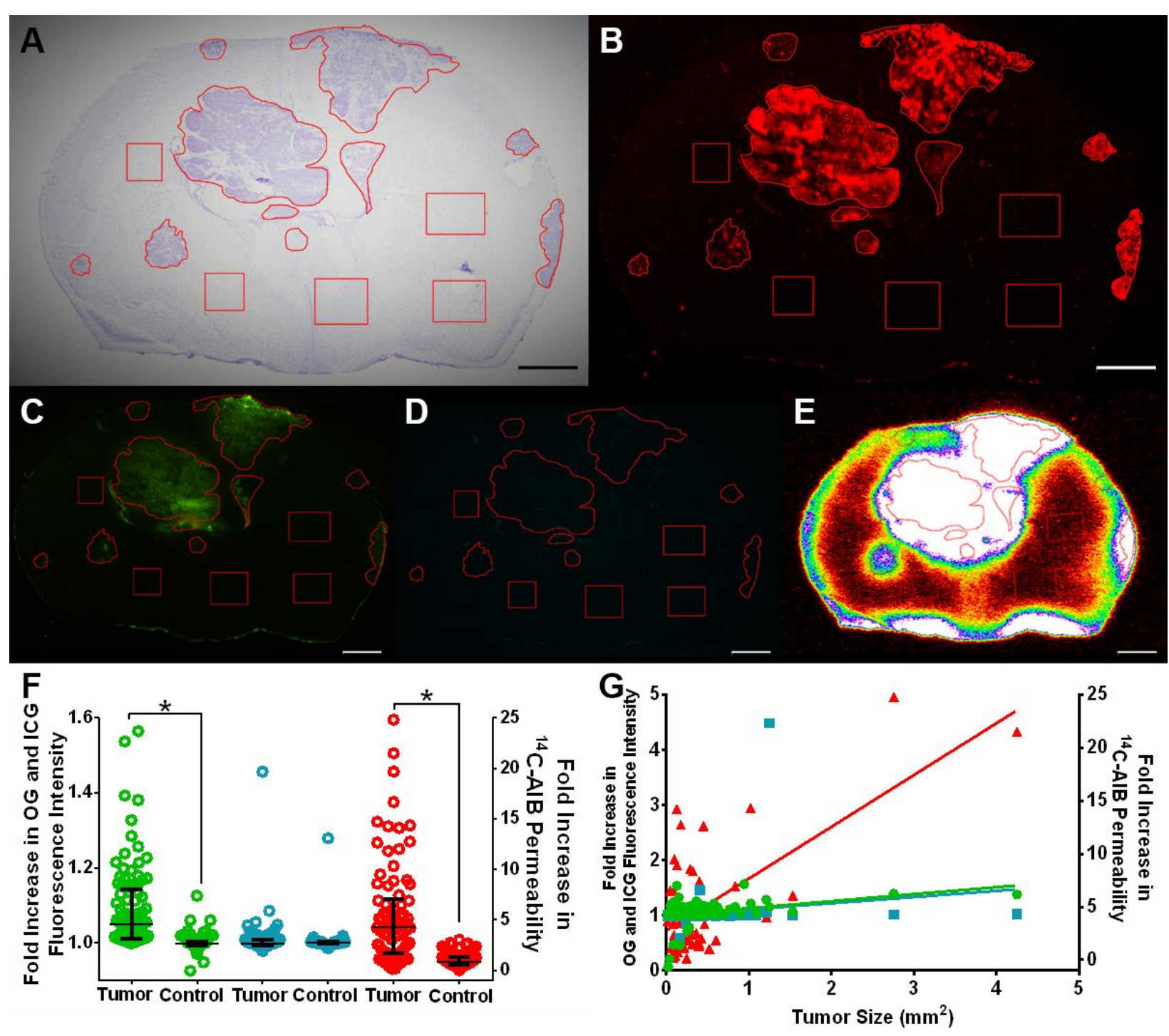

Figure 5.8. Passive permeability changes of PC-9-Br treated with cisplatinpemetrexed.

(A) A representative cresyl violet brain slice (approximately corresponding to Fig. 5.3

C3) of cisplatin-pemetrexed-treated PC-9-Br tumors, with (B) corresponding Tomato Red tumor fluorescence. (C) The same slice with Oregon Green, (D) ICG, and (E) ${ }^{14} \mathrm{C}$-AIB autoradiographic data to quantify P-gp, vascularity, and permeability increases, respectively. (F) The median and interquartile ranges for fold-increases of passive permeability markers in 96 tumors over control regions. For cisplatin-pemetrexed-treated brains, tumors were significantly more permeable to ${ }^{14} \mathrm{C}$-AIB and had more P-gp effects ( $\mathrm{p}<0.05)$, but not vascularity $(\mathrm{p}>0.05)$. (G) While the OG intensity had a modest correlation with $\mathrm{mm}^{2}\left(\mathrm{r}^{2}=0.42\right)$, ICG and ${ }^{14} \mathrm{C}$-AIB were not correlated with metastases size $\left(r^{2}<0.15\right)$. For all depicted brain slices, tumor regions are outlined while control areas are squares. Scale bar $=1 \mathrm{~mm}$. 


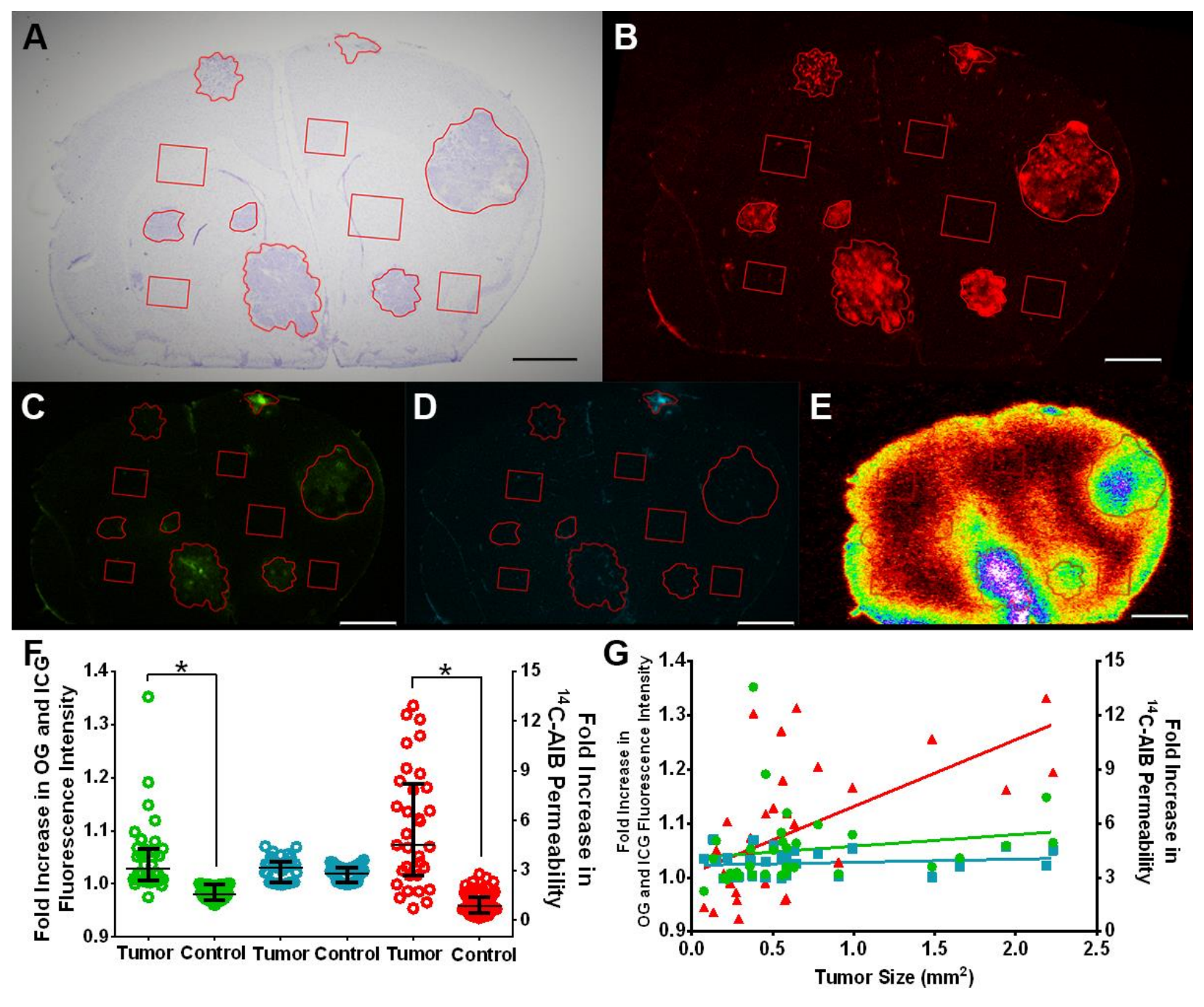

Figure 5.9. Passive permeability changes of PC-9-Br treated with gefitinib.

(A) A representative cresyl violet brain slice (approximately corresponding to Fig. 3.3

C2) of gefitinib-treated PC-9-Br tumors, with (B) corresponding Tomato Red tumor

fluorescence. (C) The same slice with Oregon Green, (D) ICG, and (E) ${ }^{14} \mathrm{C}$-AIB autoradiographic data to quantify P-gp, vascularity, and permeability increases, respectively. (F) The median and interquartile ranges for fold-increases of passive permeability markers in 34 tumors over control regions. For gefitinib-treated brains, tumors were significantly more permeable ${ }^{14} \mathrm{C}$-AIB and had more $\mathrm{P}$-gp activity $(\mathrm{p}<$ $0.05)$, but not vascularity $(\mathrm{p}>0.05)$. (G) While the permeability-fold increases of ${ }^{14} \mathrm{C}$ AIB had a modest correlation with $\mathrm{mm}^{2}\left(\mathrm{r}^{2}=0.33\right)$, OG and ICG were not correlated with metastases size $\left(r^{2}<0.13\right)$. For all depicted brain slices, tumor regions are outlined while control areas are squares. Scale bar $=1 \mathrm{~mm}$. 


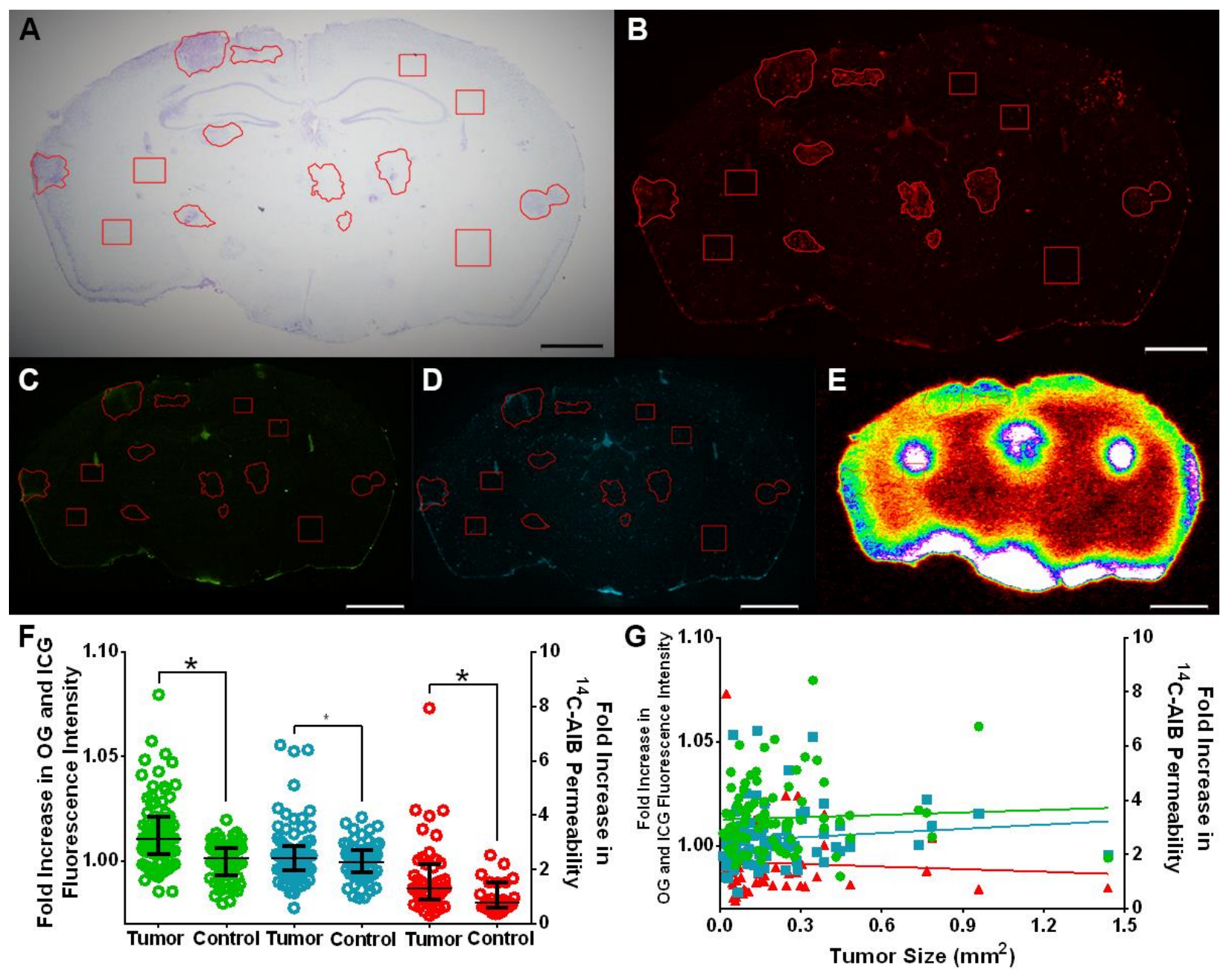

Figure 5.10. Passive permeability changes of PC-9-Br treated with afatinib.

(A) A representative cresyl violet brain slice (approximately corresponding to Fig. 5.3

C2) of afatinib-treated PC-9-Br tumors, with (B) corresponding Tomato Red tumor

fluorescence. (C) The same slice with Oregon Green, (D) ICG, and (E) ${ }^{14} \mathrm{C}$-AIB autoradiographic data to quantify P-gp, vascularity, and permeability increases, respectively. (F) The median and interquartile ranges for fold-increases of passive permeability markers in 85 tumors over control regions. For afatinib-treated brains, tumors were significantly more permeable ${ }^{14} \mathrm{C}$-AIB and had more P-gp activity and vascularity $(\mathrm{p}<0.05)$. (G) The fold increases of OG, ICG, or ${ }^{14} \mathrm{C}-\mathrm{AIB}$ were not correlated with metastases size $\left(\mathrm{r}^{2}<0.01\right)$. For all depicted brain slices, tumor regions are outlined while control areas are squares. Scale bar $=1 \mathrm{~mm}$. 


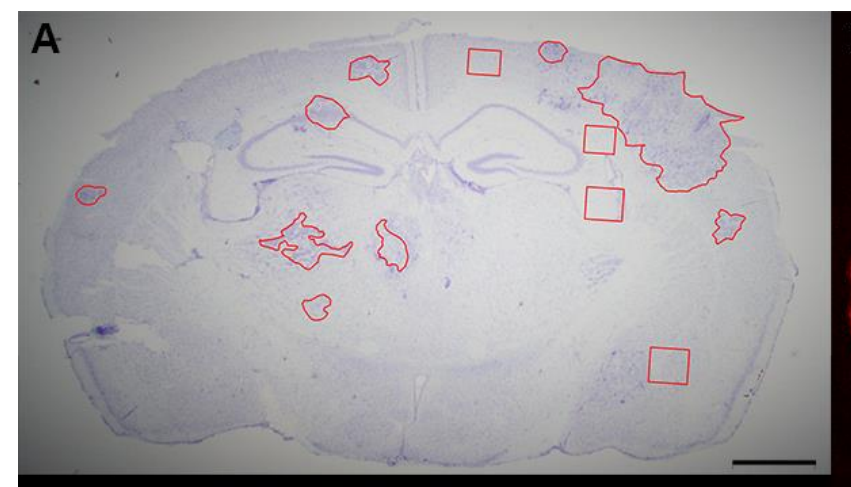

\section{B}

C

\section{D}
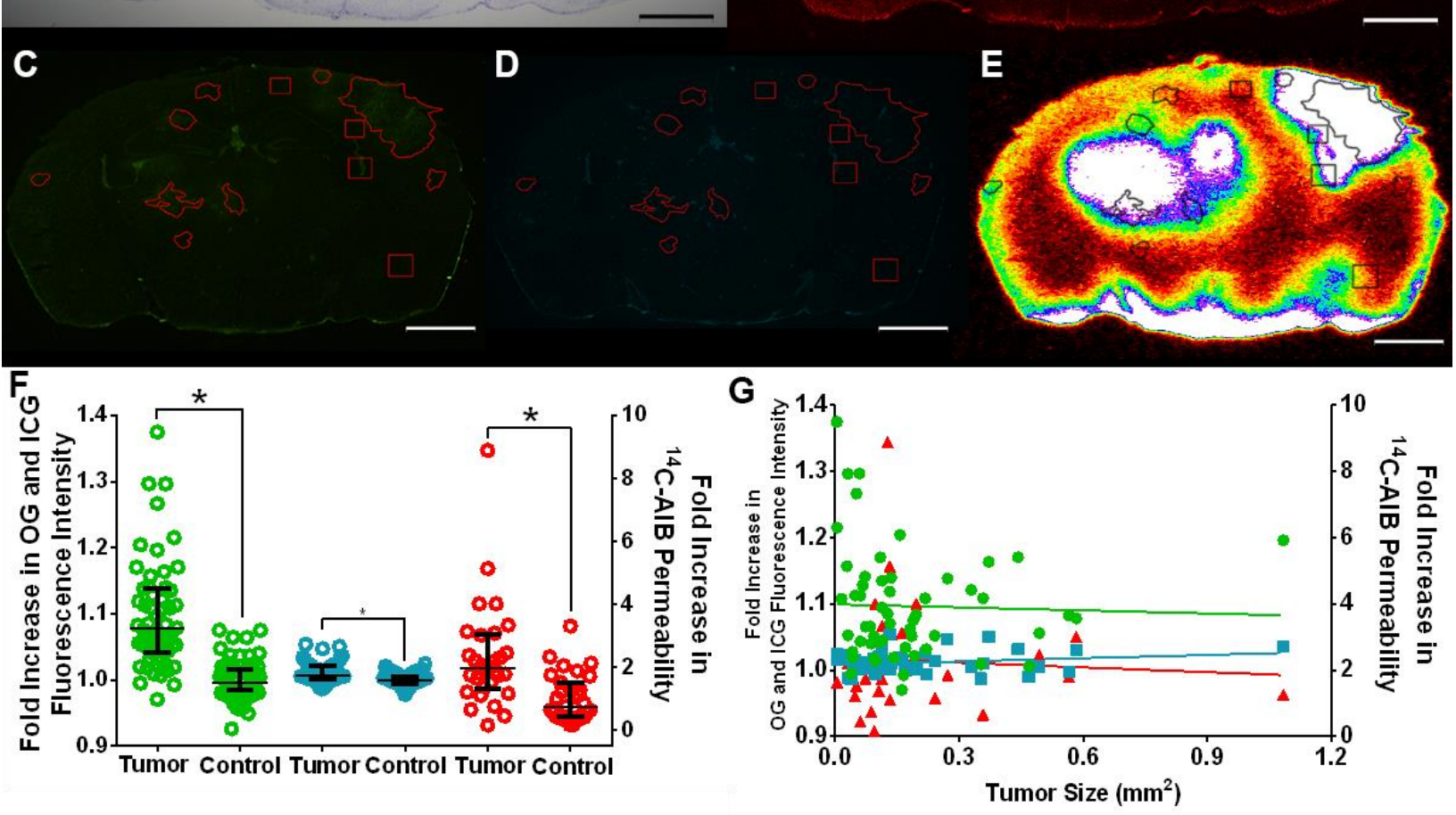

Figure 5.11. Passive permeability changes of PC-9-Br treated with osimertinib.

(A) A representative cresyl violet brain slice (approximately corresponding to Fig. 5.3

C4) of osimertinib-treated PC-9-Br tumors, with (B) corresponding Tomato Red tumor fluorescence. (C) The same slice with Oregon Green, (D) ICG, and (E) ${ }^{14} \mathrm{C}-\mathrm{AIB}$ autoradiographic data to P-gp, vascularity, and permeability increases, respectively. (F) The median and interquartile ranges for fold-increases of passive permeability markers in 56 tumors over control regions. For osimertinib-treated brains, tumors were significantly more permeable to ${ }^{14} \mathrm{C}$-AIB and had more P-gp activity and vascularity $(\mathrm{p}<0.05)$. (G) The fold increases of OG, ICG, or ${ }^{14} \mathrm{C}$-AIB were not correlated with metastases size $\left(\mathrm{r}^{2}<\right.$ 0.05). For all depicted brain slices, tumor regions are outlined while control areas are squares. Scale bar $=1 \mathrm{~mm}$. 


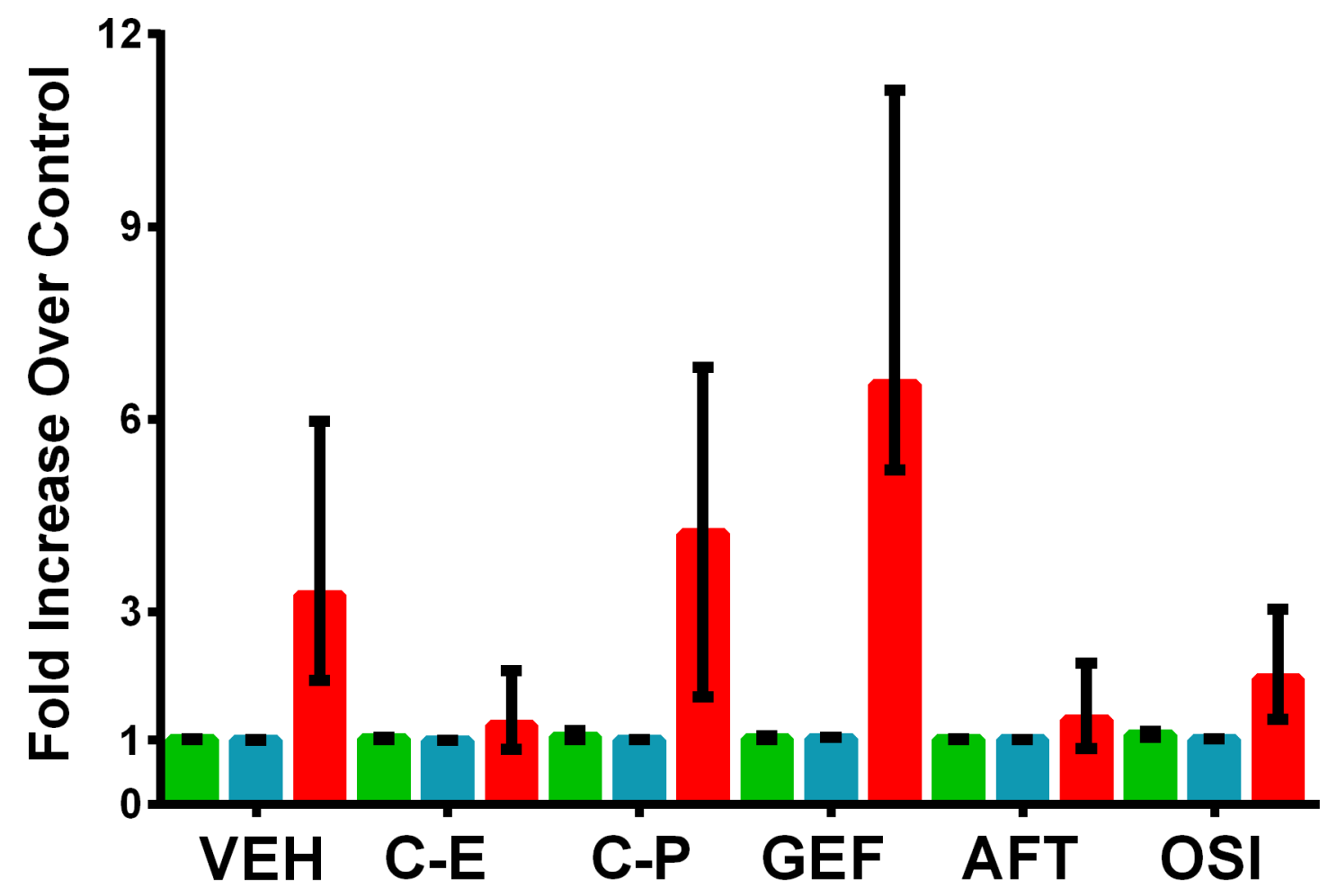

Figure 5.12. Comparison of passive permeability between PC-9-Br treatment groups.

PC-9 tumors are more permeable to the small ${ }^{14} \mathrm{C}$-AIB markers than non-tumor areas. The reduction of ${ }^{14} \mathrm{C}$-AIB permeability in cisplatin-etoposide, afatinib, and osimertinib groups compared to vehicle or cisplatin-pemetrexed groups is linked with an increase in median survival. All data represents median and interquartile ranges. AFT: afatinib; C-E: cisplatin-etoposide; C-P: cisplatin-pemetrexed; GEF: gefitinib; OSI: osimertinib; VEH: vehicle. 


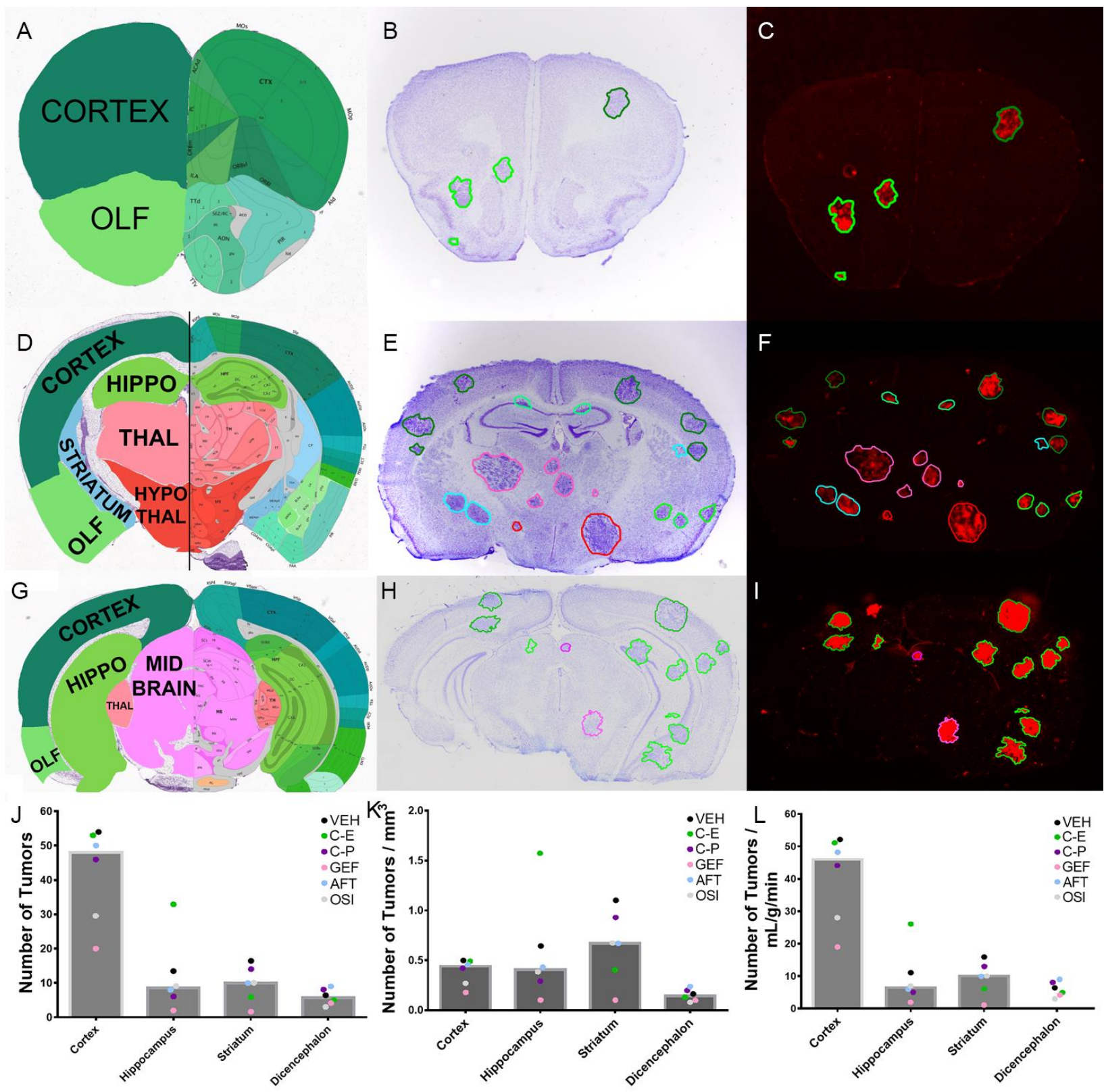

Figure 5.13. Distribution of PC-9-Br tumors.

Representative slices of (A) anterior, (D) middle, and (G) posterior slices from the Allen mouse brain atlas. Colors represent different regions of the brain. These correspond with representative $(\mathbf{B})$ anterior, $(\mathbf{E})$ middle, and $(\mathbf{H})$ posterior cresyl violet slices of brains with PC-9-Br tumors. Color outlines correspond with atlas brain regions. Tomato red tumors of $(\mathbf{C})$ anterior, (F) middle, and (I) posterior slices are similarly outlined with color. (J) A plot of the total number of tumors based on area of the brain in all treated brains. (K) A plot of the number of tumors based on surface area. (L) A plot of the number of tumors based on blood flow. Median data is plotted with colors representing different treated groups. 


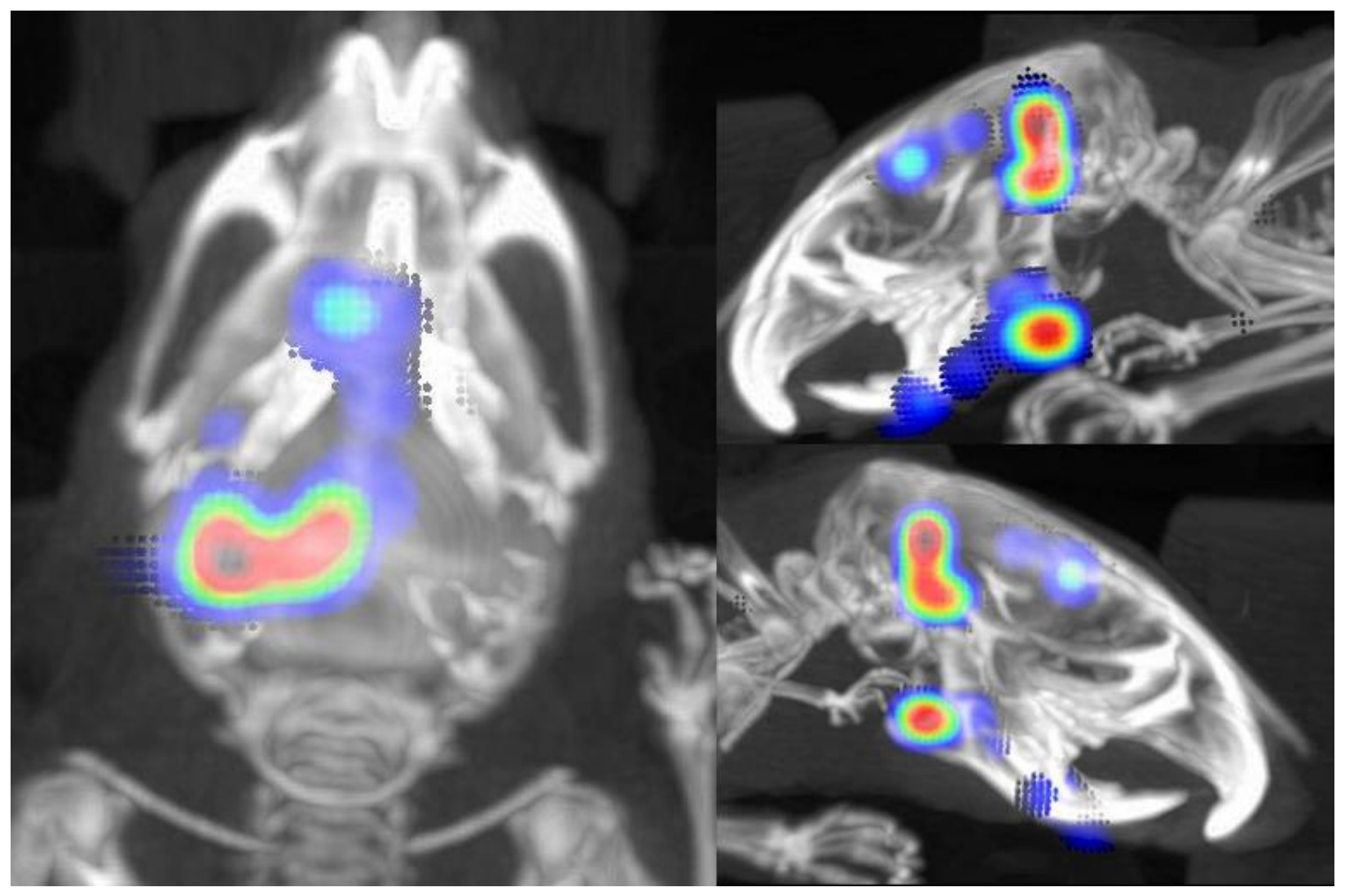

Supplemental Figure 5.1. Mandibular metastases in the PC-9-Br line.

Multiple mandibular metastases are present along with intracranial metastases characteristic of the PC-9-Br line. This distribution is seen in multiple brain-seeking metastatic cell lines, including breast cancer. 


\begin{tabular}{|c|c|c|}
\hline \multicolumn{2}{|c|}{ PC9-P } & \multirow{2}{*}{\begin{tabular}{|l|}
\multicolumn{1}{|c|}{ PC9-6-Br } \\
IC50 \pm SD
\end{tabular}} \\
\hline Compound & IC50 \pm SD & \\
\hline \multicolumn{3}{|c|}{ BEACON AGENTS } \\
\hline \multicolumn{3}{|l|}{ Gemcitabine } \\
\hline Docetaxel & & $0.2 \mathrm{nM}$ \\
\hline Eribulin & & $20.3 \mathrm{nM}$ \\
\hline Vinorelbine & & $7.1 \mathrm{nM}$ \\
\hline SN-38 & $7.7 \pm 39 \mathrm{nM}$ & $6 \pm 0.1 \mu \mathrm{M}$ \\
\hline \multicolumn{3}{|c|}{ CONVENTIONAL LUNG CHEMOTHERAPY AGENTS } \\
\hline Cisplatin & $669 \pm 653 \mathrm{~nm}$ & $593 \pm 550 \mathrm{~nm}$ \\
\hline Etoposide & $408 \pm 123 \mathrm{~nm}$ & $6.4 \pm 0.4 \mu \mathrm{M}$ \\
\hline Pemetrexed & $391 \pm 346 \mathrm{~nm}$ & $512 \pm 590 \mathrm{~nm}$ \\
\hline \multicolumn{3}{|c|}{ EGFR-TYROSINE KINASE INHIBITOR AGENTS } \\
\hline Erlotinib & $<100 \mathrm{fM}$ & $514.2 \pm 100 \mathrm{~nm}$ \\
\hline Gefitinib & $<100 \mathrm{fM}$ & $41.5 \pm 9.3 \mathrm{~nm}$ \\
\hline Afatinib & $<100 \mathrm{fM}$ & $5.2 \pm 2.1 \mathrm{~nm}$ \\
\hline Osimeritinib & $<100 \mathrm{fM}$ & $4.5 \pm 1.9 \mathrm{~nm}$ \\
\hline
\end{tabular}


Table 5.1. In-vitro chemotherapeutic sensitivity comparisons of the parenteral and brain-seeking PC-9 line.

MTT assays show that the parenteral cell lines are generally more chemosensitive than the brain-seeking line. All data is based on an $n=64$. 


\begin{tabular}{|c|l|c|c|c|}
\hline Therapy & $\mathrm{n}$ & Survival (days) & $\begin{array}{c}\text { median size } \\
\left(\mathrm{mm}^{2}\right)\end{array}$ & $\mathrm{IQR}\left(\mathrm{mm}^{2}\right)$ \\
\hline Vehicle & 114 & 42 & 0.1844 & $0.1129-0.3097$ \\
\hline $\begin{array}{c}\text { Cisplatin- } \\
\text { Etoposide }\end{array}$ & 117 & 51.5 & 0.1093 & $0.0533-0.2384$ \\
\hline Cisplatin- & 96 & 45 & 0.2492 & $0.1305-0.4054$ \\
Pemetrexed & & & & \\
\hline Gefitinib & 34 & 48 & 0.5530 & $0.2868-0.6787$ \\
\hline Afatinib & 85 & 73 & 0.1459 & $0.0687-0.2809$ \\
\hline Osimertinib & 56 & 73 & 0.1293 & $0.0629-0.2125$ \\
\hline
\end{tabular}

Table 5.2. Sizes of PC-9-Br tumors based on drug treatment.

Treatment groups that extended median survival significantly beyond vehicle (cisplatinetoposide, afatinib, and osimertinib) have median tumor sizes below $0.15 \mathrm{~mm}^{2}$. 


\begin{tabular}{|l|l|l|l|}
\hline & \multicolumn{2}{|c|}{${ }^{14} \mathrm{C}-\mathrm{AIB}$ fold increase } \\
\hline Therapy & Median & \multicolumn{1}{|c|}{ IQR } & $\mathrm{r}^{2}$ vs. size \\
\hline Vehicle & 3.254 & $1.928-5.971$ & 0.014 \\
\hline Cisplatin- & 1.231 & $0.8541-2.077$ & 0.068 \\
Etoposide & & & \\
\hline Cisplatin- & 4.235 & $1.681-7.046$ & 0.444 \\
Pemetrexed & & & \\
\hline Gefitinib & 4.537 & $2.707-8.200$ & 0.325 \\
\hline Afatinib & 1.315 & $0.8746-2.197$ & 0.004 \\
\hline Osimertinib & 1.950 & $1.328-3.033$ & 0.004 \\
& & & \\
\hline
\end{tabular}

Table 5.3. ${ }^{14} \mathrm{C}$-AIB fold permeability is less in groups that improve median survival.

In therapy that does not improve median survival (vehicle, cisplatin-pemetrexed, and gefitinib), ${ }^{14} \mathrm{C}$-AIB increases are higher than those that do improve median survival (cisplatin-etoposide, afatinib, and osimertinib). No correlation between fold increase and size exists. 


\begin{tabular}{|l|c|c|c|}
\hline & \multicolumn{2}{|c|}{ OG fold increase } \\
\hline Therapy & Median & IQR & $\mathrm{r}^{2}$ vs. size \\
\hline Vehicle & 1.007 & $1.004-1.013$ & 0.001 \\
\hline Cisplatin- & 1.020 & $1.007-1.037$ & 0.420 \\
Etoposide & & & \\
\hline Cisplatin- & 1.049 & $1.010-1.144$ & 0.123 \\
Pemetrexed & & & \\
\hline Gefitinib & 1.028 & $1.006-1.066$ & 0.030 \\
\hline Afatinib & 1.011 & $1.003-1.021$ & 0.003 \\
\hline Osimertinib & 1.077 & $1.041-1.138$ & 0.001 \\
\hline
\end{tabular}

Table 5.4. Oregon Green fluorescence intensity does not correlate with median survival improvement.

Fluorescence intensity increases in Oregon Green are similar for all treatment groups. 


\begin{tabular}{|l|c|c|c|}
\hline \multirow{2}{*}{} & \multicolumn{3}{|c|}{ ICG fold increase } \\
\hline Therapy & Median & IQR & $\mathrm{r}^{2}$ vs. size \\
\hline Vehicle & 1.000 & $0.9953-1.002$ & $<0.001$ \\
\hline Cisplatin-Etoposide & 0.9885 & $0.9810-1.001$ & 0.031 \\
\hline Cisplatin-Pemetrexed & 0.9991 & $0.9941-1.009$ & 0.030 \\
\hline Gefitinib & 1.030 & $1.002-1.042$ & 0.021 \\
\hline Afatinib & 1.001 & $0.9957-1.007$ & 0.013 \\
\hline Osimertinib & 1.006 & $1.002-1.020$ & 0.042 \\
& & & \\
\hline
\end{tabular}

Table 5.5. ICG fluorescence intensity is similar in all treatment groups.

As a measure of vascularity, ICG is similar in all groups, and is there is no correlation to size. 


\section{CHAPTER 6}

\section{EFFECTS OF ETIRINOTECAN PEGOL (NKTR-102) IN THE PC-9 PRECLINICAL LUNG CANCER METASTASES MODEL}

\subsection{Introduction}

Lung cancer is the second-most commonly diagnosed cancer in the United States, and is the most common cause of cancer death worldwide. [1, 2] It is estimated that more than 200,000 new cases of lung cancer will be diagnosed and more than 150,000 cancer deaths in the United States in 2018. [2] The average age of diagnosis is 70, while the median age of death is 72 . The short time from diagnosis to death may be due to the advanced stage on presentation. [3]

Lung cancer metastasizes to the brain in approximately 10 to $30 \%$ of patients and is responsible for the majority of brain metastases. [4] The propensity to form brain metastases is also influenced by lung cancer subtype. Approximately $85 \%$ of lung cancer are non-small cell lung carcinoma (NSCLC) with small-cell lung carcinoma (SCLC) comprising the rest. [5] SCLC presents with brain metastases in $20 \%$ of patients, forming in $80 \%$ within 2 years of diagnosis. [6] Adenocarcinoma, the most common subtype of NSCLC, presents with brain metastases in $10 \%$ of patients, forming in $40 \%$ throughout illness progression. [3] Within adenocarcinoma, the most common mutation is $K R A S$, followed by EGFR and EMLA-ALK translocation. Targetable drugs exist for EGFR and EML4-ALK, but not for KRAS.

Therapeutic options in the treatment of lung cancer brain metastases (LCBM) include surgical resection, stereotactic radiosurgery, whole brain radiotherapy, and 
chemotherapy. [7] Even when used in combination, these options rarely improve survival to over 12 months [8] and are thus often palliative. Incremental improvements have been made in treating brain metastases, especially in creating targeted agents and repurposing nonspecific chemotherapy. The presence of the blood-brain barrier (BBB) and bloodtumor barrier (BTB) can significantly hinder penetration of chemotherapeutic agents into both tumor and brain tissue. [9]

The BBB consists of a physical barrier of vascular endothelial cells linked together by tight junctions, enzymes such as phosphatases to degrade substances, and efflux transports actively restricting molecular entry into the brain, all surrounded by astrocytic foot processes performing similar activities. [10] In the BTB, immature vasculature structure leads to increased permeability and though drug permeation is enhanced, the magnitude of enhancement often falls below therapeutic amounts required for efficacy. [9]

Due to its prevalence in LCBM and in vitro sensitivity to SN-38, the EGFRmutant PC-9 cell line was created into a brain-seeking line and tested for preclinical survival efficacy of NKTR-102 against BEACON-agents, conventional lung chemotherapy, and targeted EGFR inhibitors. Herein, we report that NKTR-102 did not reduce tumor burden or improve survival significantly from vehicle, BEACON-agents, gefitinib, or the cisplatin-pemetrexed combination lung therapy. Conversely, cisplatinetoposide and the targeted inhibitors afatinib and osimertinib improved survival with most animals surviving until trial completion for the latter two agents.

\subsection{Methods and Materials}

\section{Animals}


The animal protocol used is the same as in Chapter 3.2.

\section{Cells}

The PC-9 parenteral line was provided by Dr. Lori Hazlehurst pre-transduced with the pcDNA3.1(+)/Luc2=tdT plasmid. These cells were serially intracardially injected and extracted through brains six times to create the PC-9-Br line. Cells were grown in a $37 \mathrm{C}$ incubator at $5 \% \mathrm{CO}_{2}$, and grown in RPMI with $10 \%$ fetal bovine serum, 5\% penicillin-streptomycin, and $10-11 \mu \mathrm{L} / \mathrm{mL}$ of G4918 (Geneticin) to ensure plasmid expression. All cells used in this experiment are between passages 1 to 10 . A549 and CCL-185IG were acquired from American Type Culture Collection (ATCC). A549 has a KRAS mutation while CCL-185IG is a modified A549 to express the EML4-ALK fusion peptide. Hence, A549 will be termed A549N, while CCL-185IG will be termed A549F.

\section{Stereotactic injection}

The protocol for intracardiac cell injection is the same as in Chapter 4.2.

\section{Drugs and dosing}

The dosing regimen and solubility of cisplatin, etoposide, pemetrexed, gefitinib, afatinib, and osimertinib are the same as in section 4.2. The dosing regimen and solubility for irinotecan, eribulin, docetaxel, vinorelbine, gemcitabine, and NKTR-102 are the same as in Chapter 4.2. These are summarized in Table 6.1.

\section{Longitudinal bioluminescence}

Animals were given an intraperitoneal $150 \mathrm{mg} / \mathrm{kg}$ injection of d-luciferin potassium salt and anesthetized with $2 \%$ isoflurane. Based on the results from Chapter $\mathbf{3}$, 
after 10 minutes of circulation, animals were transferred to the IVIS Spectra CT (PerkinElmer) and bioluminescence (BLI) was captured at auto-exposure and one-minute time spans on Stage D with medium binning, fitting within the optimal imaging time for the PC-9-Br line (Figure 4.2). BLI was taken twice weekly to measure chemotherapy response and tumor burden, performed at least an hour prior to drug administration to avoid interactions. For quantification, a region of interest (ROI) was drawn based on cranial circumference. BLI based on ROI is reported as radiance (photons $/ \mathrm{sec} / \mathrm{cm}^{2} /$ steridian).

\section{Toxicology}

MTT in vitro toxicity studies were performed using the same protocol as in

\section{Chapter 4.2.}

\section{Statistics}

On survival endpoints, mice were sacrificed and date of death recorded. KaplanMeier curves were generated and compared using log-rank statistics. All statistics were performed on GraphPad Prism 6.0 and considered significant at $\mathrm{p}<0.05$.

\subsection{Results}

\section{PC-9-Br shows the most SN-38 sensitivity of the developing LCBM lines}

In order to determine which cell line to conduct NKTR-102 in vivo, an in vitro toxicology study was performed on parenteral lung cancer cells using NKTR-102's active metabolite SN-38. The EGFR-mutant PC-9 showed the highest sensitivity to SN-38 at 7.3 nM, while the KRAS mutant A549N and ELM4-ALK-expressing A549F were $979 \mathrm{nM}$ and $6.2 \mu \mathrm{M}$, respectively (Fig. 6.1). PC-9-Br was chosen as the model for the NKTR-102 
efficacy study, along with targeted agents against the exon 19 deleted EGFR receptor it possesses. Subsequently, a brain-seeking line was created through 6 passages of injections and extractions.

\section{Non-targeted chemotherapy does not significantly prolong survival of PC-9}

\section{brain metastases compared to vehicle}

The main focus of the study was to evaluate the efficacy of NKTR-102 compared to BEACON-specific agents, conventional lung cancer chemotherapy, and targeted chemotherapy in the experimental LCBM model. For grouping purposes, outcomes will be reported as "non-targeted" (e.g. NKTR-102, BEACON agents, and lung chemotherapy) and "targeted" (e.g. EGFR inhibitors). After the injection of 150,000 PC-

9-Br cells into the left ventricle, metastatic lesions were allowed to grow for 21 days prior to drug administration and BLI measurements. Thus, 21 days (Week 3) is the baseline to normalize further values. BLI was taken twice weekly as a quantitative surrogate for tumor burden and chemotherapeutic efficacy.

In vehicle PC-9-Br animals (Fig. 6.2A), tumor burden increased by 29,400-fold from baseline across 8 weeks (Fig. 6.2B). Similarly, gemcitabine increased 28,400-fold, eribulin increased 14,800-fold, docetaxel increased 8000-fold, and vinorelbine increased 400-fold above baseline, and NKTR-102 signal increased by 1870-fold (Fig. 6.2B). The BEACON-agents and NKTR-102 featured almost identical increases in tumor burden for 6 weeks after injection, then separated as survivors who bore lower BLI populated the curve (Fig. 6.2B). The similarities of BLI curves are also represented in survival curves. Vehicle mice survived for a median of 42 days, with irinotecan-treated mice surviving for a median of 42 days, gemcitabine mice surviving for a median of 45.5 days, docetaxel 
mice surviving for a median of 42.5 days, eribulin mice surviving for a median of 43.5 days, vinorelbine mice surviving for a median of 41 days, and NKTR-102 mice surviving for a median of 43 days (Fig. 6.3). No significance in median survival was seen with BEACON-agents or NKTR-102 compared to vehicle $(\mathrm{p}<0.05)$ (Table 6.2).

For conventional lung chemotherapy (Fig. 6.4A), cisplatin and pemetrexedtreated mice had a 4400-fold increase in BLI (Fig. 6.4B) and survived for 45 days (Fig. 6.5), while cisplatin and etoposide administration increased tumor BLI by 2700-fold (Fig. 6.4B) and significantly extended median survival to 51.5 days after injection compared to vehicle ( $\mathrm{p}<0.05)$ (Fig. 6.5). The cisplatin and etoposide combination is the only nontargeted chemotherapy to significantly extend survival (Table 6.2). Two eribulin mice and one NKTR-102 mouse responded to therapy, with the latter excluded from Fig. 6.2B due to significantly lowered bioluminescence values. The responder curve is attached as a supplementary figure $(\mathbf{S 1})$.

\section{Targeted second-and third-generation tyrosine kinase inhibitors significantly} prolongs survival in PC-9-Br mice compared to vehicle

EGFR-targeted inhibitors were administered daily via oral gavage to mice (Fig. 6.6A). In gefitinib-treated mice, BLI rose in a similar fashion to vehicle-treated mice albeit much lower, with a maximum 1200-fold above baseline (Fig. 6.6B). Gefitinib median survival was 48 days, which was not significant compared to vehicle $(\mathrm{p}<0.05)$ (Fig. 6.7). In contrast, daily administration of the EGFR-inhibitors afatinib and osimertinib led to an early and sustained response compared to non-targeted chemotherapy and gefitinib. BLI was increased by 100 -fold in the afatinib-treated mice (Fig. 6.6B) and 8 of 9 mice survived until the end of the trial (Fig. 6.7), and in the 
osimertinib-treated group tumor burden increased 300-fold compared to baseline (Fig. 6.6B) with 8 of 9 mice surviving until the end of the trial (Fig. 6.7). Both afatinib and osimertinib significantly increased survival compared to vehicle (Table 6.2).

\subsection{Discussion}

Significant variability exists of a chemotherapy entering peripheral tissue vs. the brain. This may be due to the brain being a "sanctuary site" for metastases, due to the presence of the blood-brain and blood-tumor barriers.

Platinum-based therapy, including cisplatin-etoposide and cisplatin-pemetrexed, have shown limited efficacy in multiple Phase II trials involving EGFR-mutated LCBM. [8] Platinum doublet therapy has largely been replaced by the use of targeted inhibitors.

It was shown that gefitinib was superior to carboplatin-pemetrexed therapy in prolonging progression-free survival in EGFR-mutated brain metastases. [11] Despite being substrates for P-glycoprotein efflux, it was demonstrated that erlotinib [12] and gefitinib [13] enter the brain metastatic parenchyma and numerous case reports depict prolonged survival and positive outcomes using these first-line $E G F R$-tyrosine kinase inhibitors. [14, 15] Similarly, afatinib is a substrate for P-glycoprotein [16] and has shown some clinical benefit in the LUX-Lung 3 and LUX-Lung 6 trials, where there was an overall 2.8-month increase in progression-free survival. [17] Osimertinib, the only third-generation EGFR TKI, has been shown to penetrate and accumulate in brain tissue, [13] with the FLAURA trial showing osimertinib having a superior progression-free survival compared to standard of care. [18] 
It is unclear as to why NKTR-102 did not show an improvement in median survival compared to either other non-targeted agents or targeted inhibitors. NKTR-102 is shown to penetrate the blood-tumor barrier and accumulate in tumors to provide a sustained cytotoxic exposure to the topoisomerase I inhibitor SN-38. [19] NKTR-102 improved survival in two models of BCBM (Chapter 4) compared to BEACON therapy and preliminary evidence shows its efficacy in the glioblastoma model (Chapter 7). The use of irinotecan in small cell lung cancer brain metastases is documented, though its use in NSCLC brain metastases is not documented.

It is hypothesized that NKTR-102 works best in large, slow-growing tumors. Median survival for PC-9-Br in this study (42 days) is similar to the median survival for the MDA-MB-231-Br (38 days) and longer than the JIMT-1-Br (28 days) BCBM vehicle groups (Chapter 4). PC-9-Br tumors are smaller and more numerous than either BCBM tumor types, which may limit the ability of NKTR-102 to sufficiently act in a depot fashion.

It is also unclear why gefitinib failed to improve median survival in this preclinical model when reports of its efficacy exists in the clinical setting. It is possible that the dose used was insufficient to penetrate into tumor parenchyma. A recent paper utilizing gefitinib, afatinib, and osimertinib in the PC-9 model of brain metastases was published and the doses inside are used in our model. In this paper, gefitinib was dosed at $6.25 \mathrm{mg} / \mathrm{kg}$ and showed no effect on improving median survival compared to control [13] while another paper utilized gefitinib at $40 \mathrm{mg} / \mathrm{kg}$ in their preclinical PC-9 brain tumor model and showed great improvements in median survival compared to vehicle therapy. [20] Similarly, afatinib was dosed at $7.5 \mathrm{mg} / \mathrm{kg}$ [13] while another paper utilized afatinib 
at 15 to $30 \mathrm{mg} / \mathrm{kg}$ in their preclinical PC-9 brain tumor model. [16] We opted to use the higher afatinib dosing but performed our study prior to the higher gefitinib dosing publication, which if followed may have had vastly different results.

\subsection{Conclusion}

While etirinotecan pegol shows efficacy in preclinical models of breast-brain metastases and glioblastoma, it does not improve median survival compared to vehicle or conventional chemotherapy in a preclinical EGFR-mutated lung cancer model. The second- and third-generation tyrosine kinase inhibitors show early tumor burden control which eventually shows resistance, mimicking the effects of continuous dosing seen in clinical settings. 


\subsection{References}

1. Wong, M.C.S., et al., Incidence and mortality of lung cancer: global trends and association with socioeconomic status. Sci Rep, 2017. 7(1): p. 14300.

2. Siegel, R.L., K.D. Miller, and A. Jemal, Cancer Statistics, 2017. CA Cancer J Clin, 2017. 67(1): p. 7-30.

3. Ali, A., et al., Survival of patients with non-small-cell lung cancer after a diagnosis of brain metastases. Curr Oncol, 2013. 20(4): p. e300-6.

4. Niemiec, M., et al., Characteristics of long-term survivors of brain metastases from lung cancer. Rep Pract Oncol Radiother, 2011. 16(2): p. 49-53.

5. Zappa, C. and S.A. Mousa, Non-small cell lung cancer: current treatment and future advances. Transl Lung Cancer Res, 2016. 5(3): p. 288-300.

6. Lekic, M., et al., Outcome of small cell lung cancer (SCLC) patients with brain metastases in a routine clinical setting. Radiol Oncol, 2012. 46(1): p. 54-9.

7. Chi, A. and R. Komaki, Treatment of brain metastasis from lung cancer. Cancers (Basel), 2010. 2(4): p. 2100-37.

8. Cedrych, I., et al., Systemic treatment of non-small cell lung cancer brain metastases. Contemp Oncol (Pozn), 2016. 20(5): p. 352-357.

9. Lockman, P.R., et al., Heterogeneous blood-tumor barrier permeability determines drug efficacy in experimental brain metastases of breast cancer. Clin Cancer Res, 2010. 16(23): p. 5664-78.

10. Daneman, R. and A. Prat, The blood-brain barrier. Cold Spring Harb Perspect Biol, 2015. 7(1): p. a020412. 
11. Patil, V.M., et al., Phase III study of gefitinib or pemetrexed with carboplatin in EGFR-mutated advanced lung adenocarcinoma. ESMO Open, 2017. 2(1): p. $\mathrm{e} 000168$.

12. Weber, B., et al., Erlotinib accumulation in brain metastases from non-small cell lung cancer: visualization by positron emission tomography in a patient harboring a mutation in the epidermal growth factor receptor. J Thorac Oncol, 2011. 6(7): p. 1287-9.

13. Ballard, P., et al., Preclinical Comparison of Osimertinib with Other EGFR-TKIs in EGFR-Mutant NSCLC Brain Metastases Models, and Early Evidence of Clinical Brain Metastases Activity. Clin Cancer Res, 2016. 22(20): p. 5130-5140.

14. Bai, H., L. Xiong, and B. Han, The effectiveness of EGFR-TKIs against brain metastases in EGFR mutation-positive non-small-cell lung cancer. Onco Targets Ther, 2017. 10: p. 2335-2340.

15. Baik, C.S., M.C. Chamberlain, and L.Q. Chow, Targeted Therapy for Brain Metastases in EGFR-Mutated and ALK-Rearranged Non-Small-Cell Lung Cancer. J Thorac Oncol, 2015. 10(9): p. 1268-1278.

16. Zhang, S.R., et al., Efficacy of afatinib, an irreversible ErbB family blocker, in the treatment of intracerebral metastases of non-small cell lung cancer in mice. Acta Pharmacol Sin, 2017. 38(2): p. 233-240.

17. Schuler, M., et al., First-Line Afatinib versus Chemotherapy in Patients with Non\&\#x2013;Small Cell Lung Cancer and Common Epidermal Growth Factor Receptor Gene Mutations and Brain Metastases. Journal of Thoracic Oncology. 11(3): p. 380-390. 
18. Vansteenkiste J, e.a., CNS Response to Osimertinib Vs. Standard-of-Care EGFRTKI as First-Line Treatment in Patients with EGFRm Advanced NSCLC: FLAURA. . ESMO Asia 2017 Congress, 2017.

19. Adkins, C.E., et al., NKTR-102 Efficacy versus irinotecan in a mouse model of brain metastases of breast cancer. BMC Cancer, 2015. 15: p. 685.

20. Tan, J., et al., Tyrosine kinase inhibitors show different anti-brain metastases efficacy in NSCLC: A direct comparative analysis of icotinib, gefitinib, and erlotinib in a nude mouse model. Oncotarget, 2017. 8(58): p. 98771-98781. 


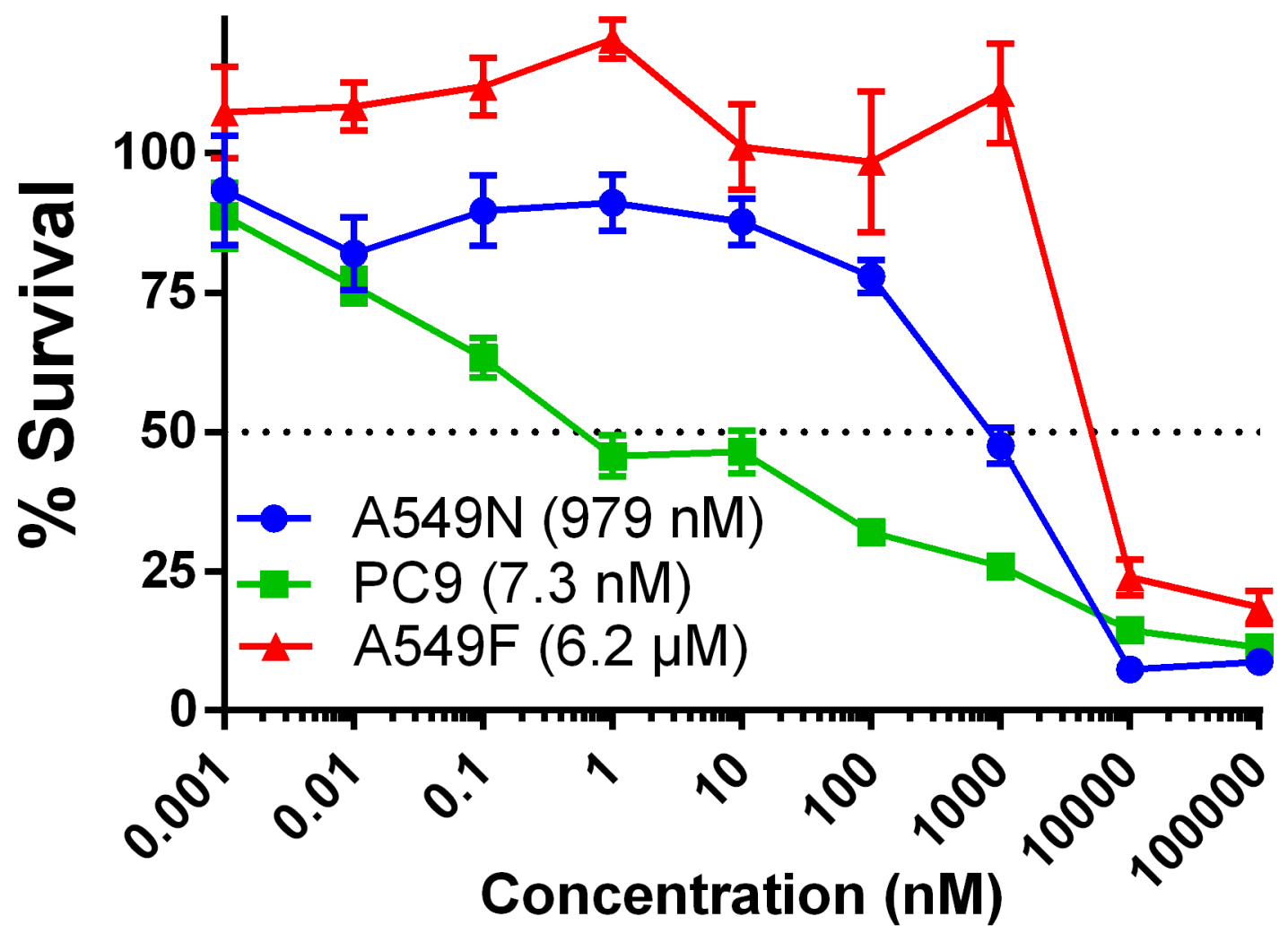

Figure 6.1. Sensitivity to SN-38 for three brain-seeking lung cancer lines.

Increasing concentrations of SN-38 were used to create a cytotoxicity assay curve. The EGFR-mutant PC-9 shows the greatest sensitivity to SN-38. Data presented is based on an $n=96$ and presented as mean \pm SEM. 


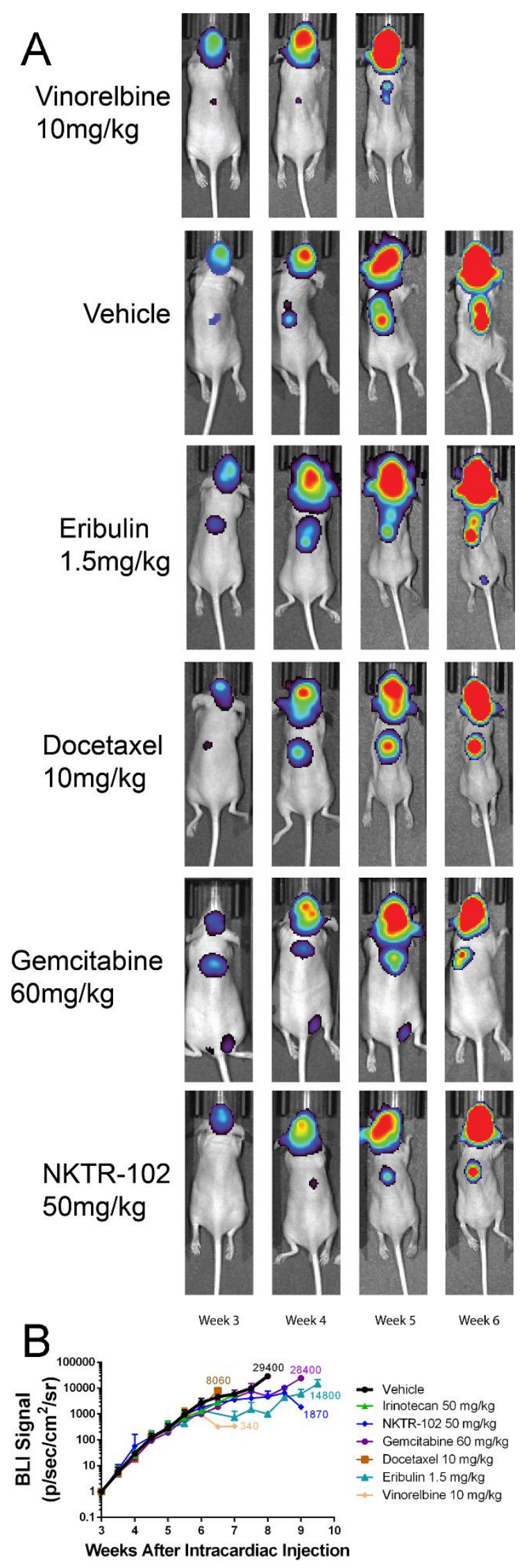


Figure 6.2. Bioluminescence in PC-9-Br treated with BEACON therapy.

(A) BEACON-treated mice do not stop tumor growth in PC-9-Br cells. (B)

Bioluminescence values in vehicle-treated mice is similar to that of BEACON therapy. 


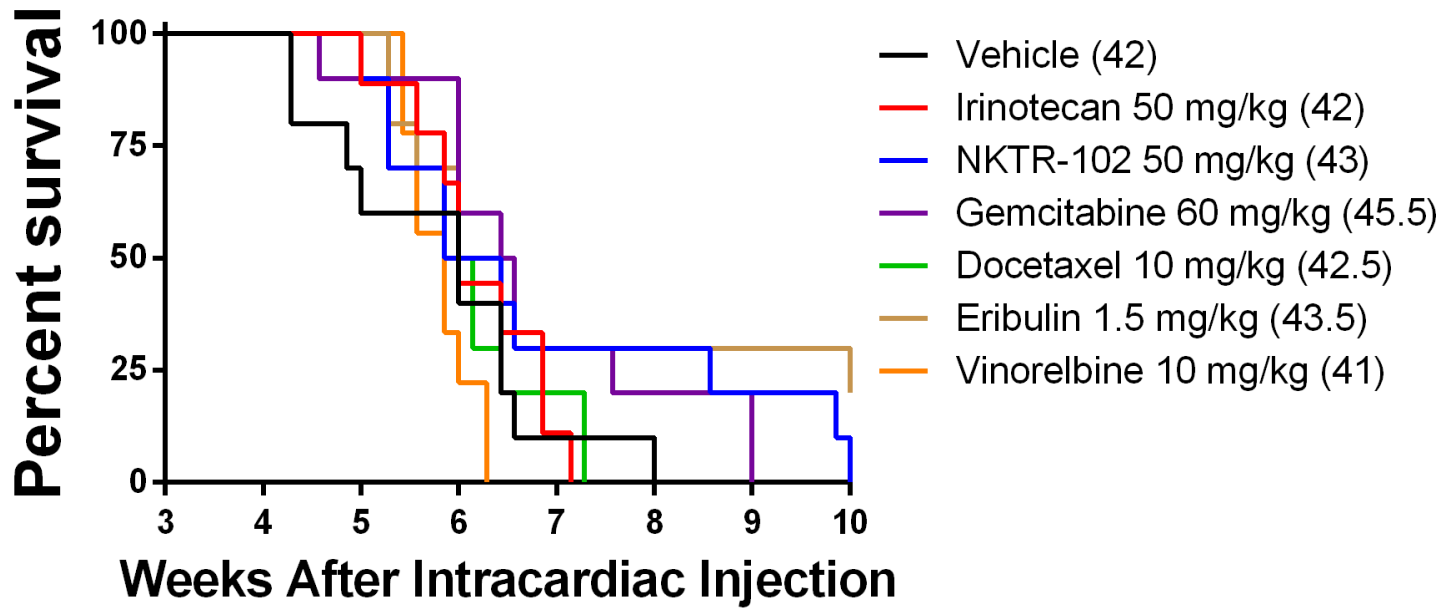

Figure 6.3. Kaplan-Meier survival curve of PC-9-Br mice treated with etirinotecan pegol and BEACON-based chemotherapy.

On day 21 after intracardiac injection of PC-9-Br cells, mice were treated with vehicle (saline, $\mathrm{n}=10)$, docetaxel $(10 \mathrm{mg} / \mathrm{kg}, \mathrm{n}=10)$, vinorelbine $(10 \mathrm{mg} / \mathrm{kg}, \mathrm{n}=9)$, eribulin $(1.5$ $\mathrm{mg} / \mathrm{kg}, \mathrm{n}=10)$, gemcitabine $(60 \mathrm{mg} / \mathrm{kg}, \mathrm{n}=10)$, irinotecan $(50 \mathrm{mg} / \mathrm{kg}, \mathrm{n}=10)$ or etirinotecan pegol $(50 \mathrm{mg} / \mathrm{kg}, \mathrm{n}=10)$. Median survival time were 42 days for vehicle, 42.5 days for docetaxel, 41 days for vinorelbine, 43.5 days for eribulin, 45.5 days for gemcitabine, 42 days for irinotecan, and 43 days for etirinotecan pegol. Etirinotecan pegol did not improve median survival compared to vehicle or other BEACON chemotherapy $(\mathrm{p}>0.05)$. Data was analyzed using log-rank statistics. 


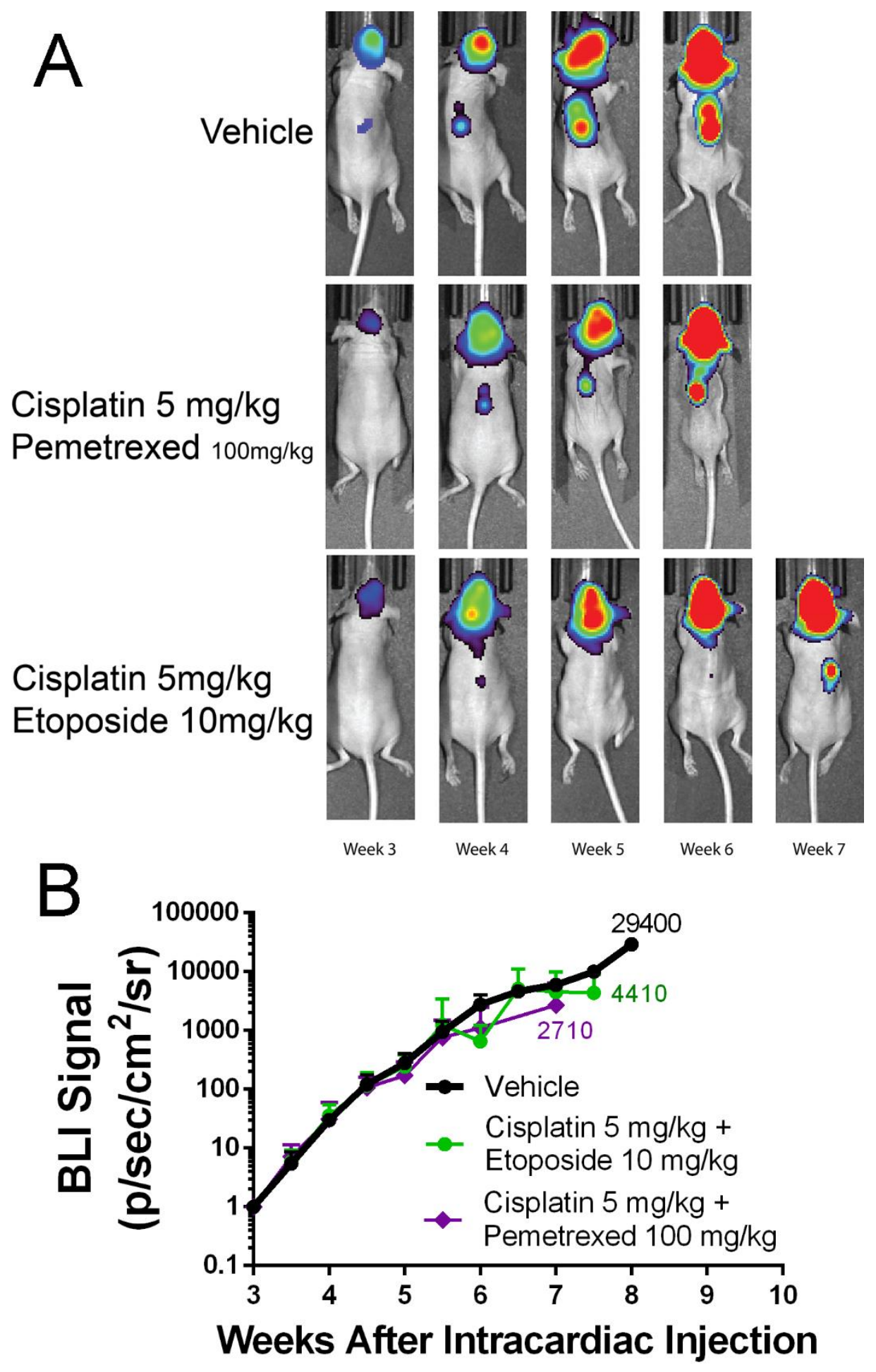


Figure 6.4. Bioluminescence in conventional lung chemotherapy in the PC-9-Br model.

(A) Longitudinal imaging shows tumor burden increases similarly in vehicle- and cisplatin-pemetrexed-treated mice, while cisplatin-etoposide has slightly better tumor burden control. 


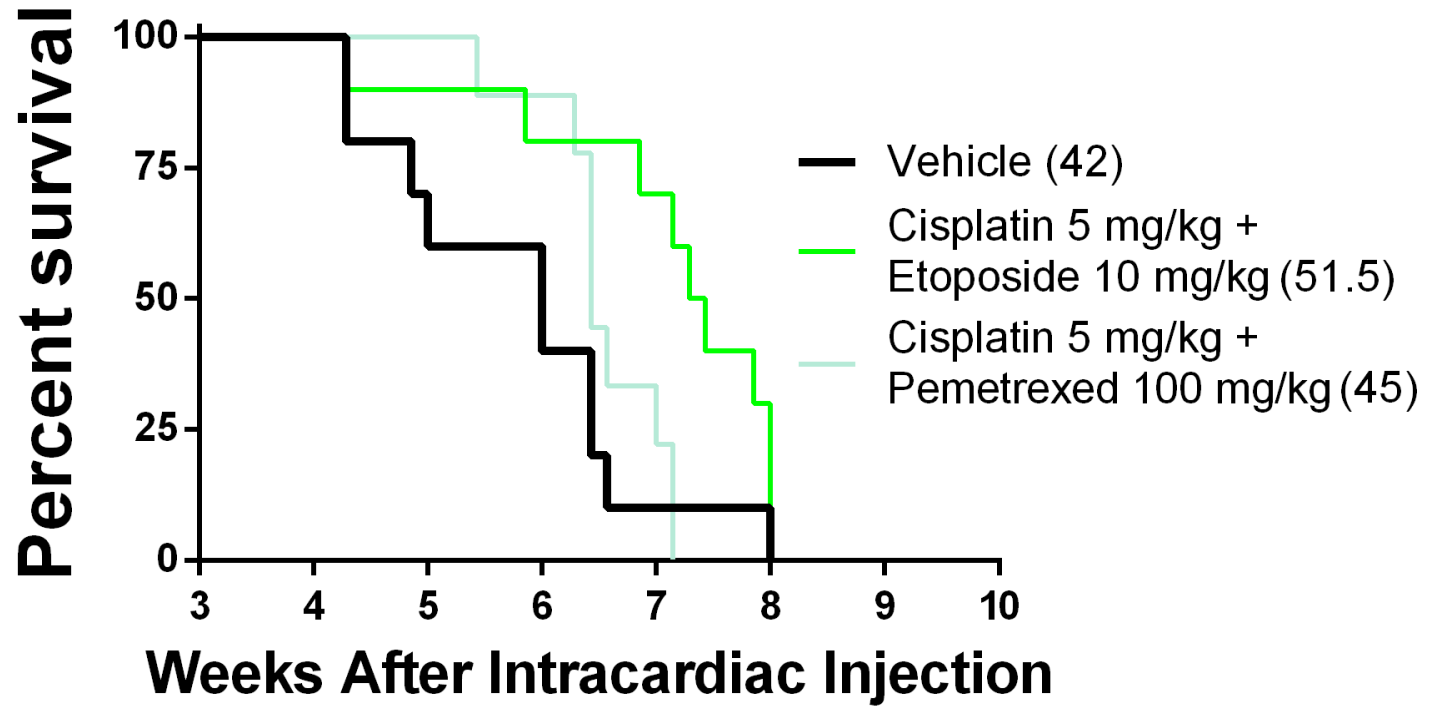

Figure 6.5. Kaplan-Meier survival curve of PC-9-Br mice treated with conventional lung chemotherapy.

On day 21 after intracardiac injection of PC-9-Br cells, mice were treated with vehicle (saline, $n=10)$, combined cisplatin-etoposide $(n=10)$, or combined cisplatin-pemetrexed $(\mathrm{n}=9)$. Median survival time was 42 days for vehicle, 51.5 days for cisplatin-etoposide, and 45 days for cisplatin-pemetrexed. Cisplatin-etoposide significantly improved median survival compared to vehicle $(\mathrm{p}<0.05)$, though cisplatin-pemetrexed did not $(\mathrm{p}>0.05)$. All data was analyzed using log-rank statistics. 


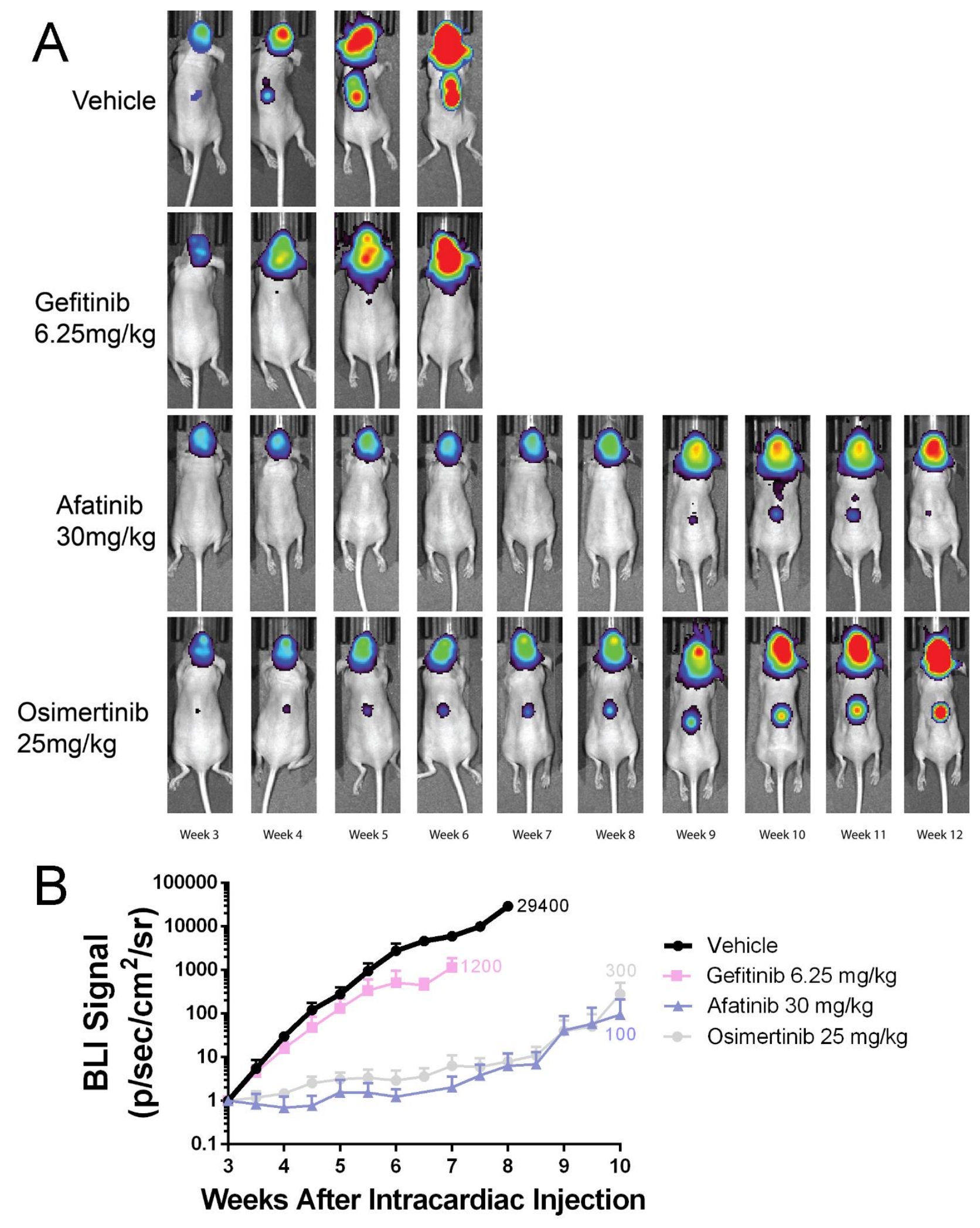


Figure 6.6. Bioluminescence profiles of EGFR-inhibitors in the PC-9-Br model.

(A) While gefitinib does not control tumor burden compared to vehicle therapy, afatinib and osimertinib control tumor burden until the end of the trial. (B) All targeted inhibitors show reduced bioluminescence signaling compared to vehicle therapy, though afatinib and osimertinib show early and sustained tumor control, with drug resistance occurring at the end of the trial. 


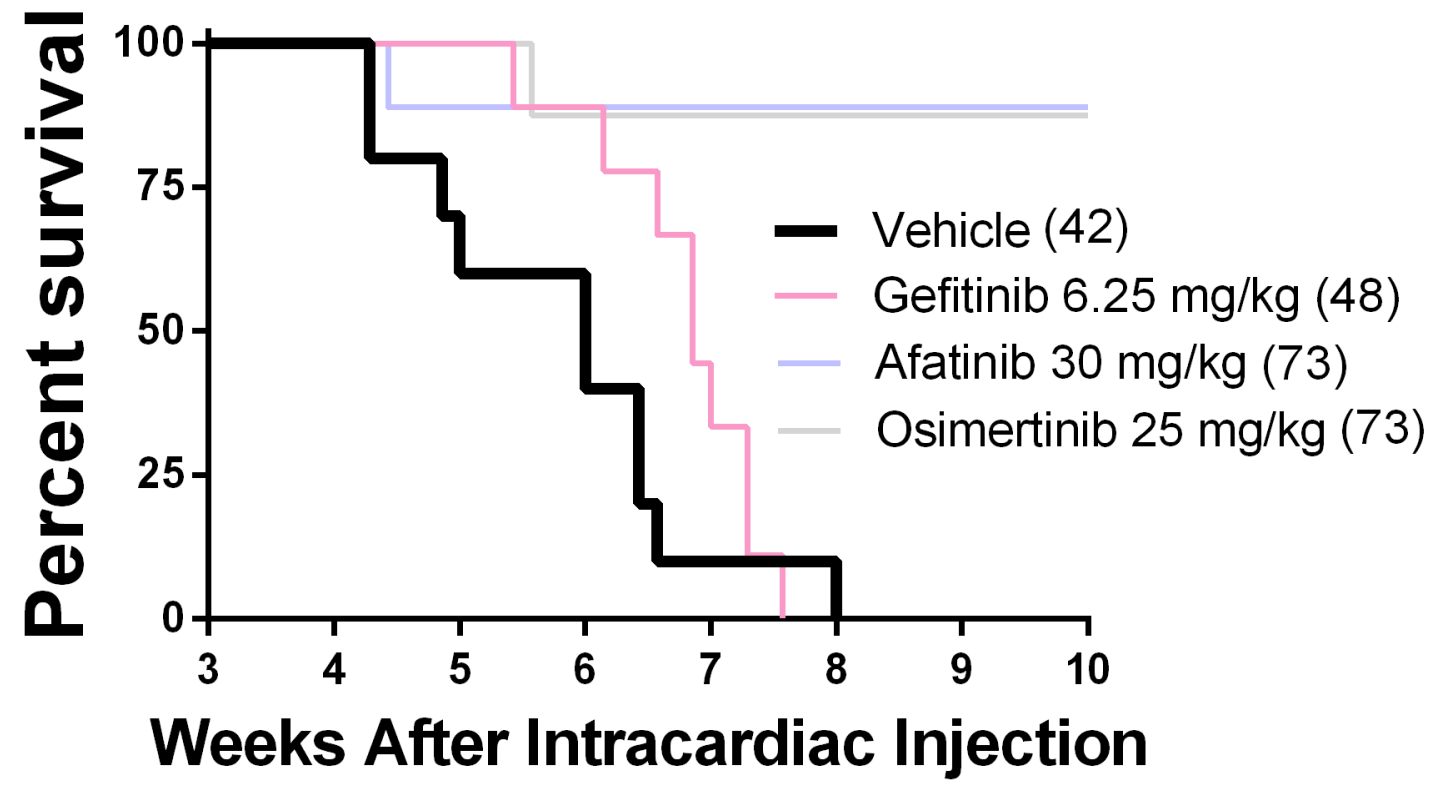

Figure 6.7. Kaplan-Meier survival curve of PC-9-Br mice treated with targeted lung chemotherapy.

On day 21 after intracardiac injection of PC-9-Br cells, mice were treated with vehicle (saline, $n=10)$, gefitinib $(n=9)$, afatinib $(n=9)$, or osimertinib $(n=8)$. Median survival time was 42 days for vehicle, 48 days for gefitinib, and undefined for afatinib and osimertinib. One mouse each died in the afatinib and osimertinib groups not due to tumor or drug toxicity, and were counted in the statistics. Gefitinib did not improve median survival compared to vehicle ( $\mathrm{p}>0.05$ ), but both afatinib and osimertinib significantly improved median survival $(\mathrm{p}<0.05)$ with all but one mouse surviving until the end of the trial in both groups. 


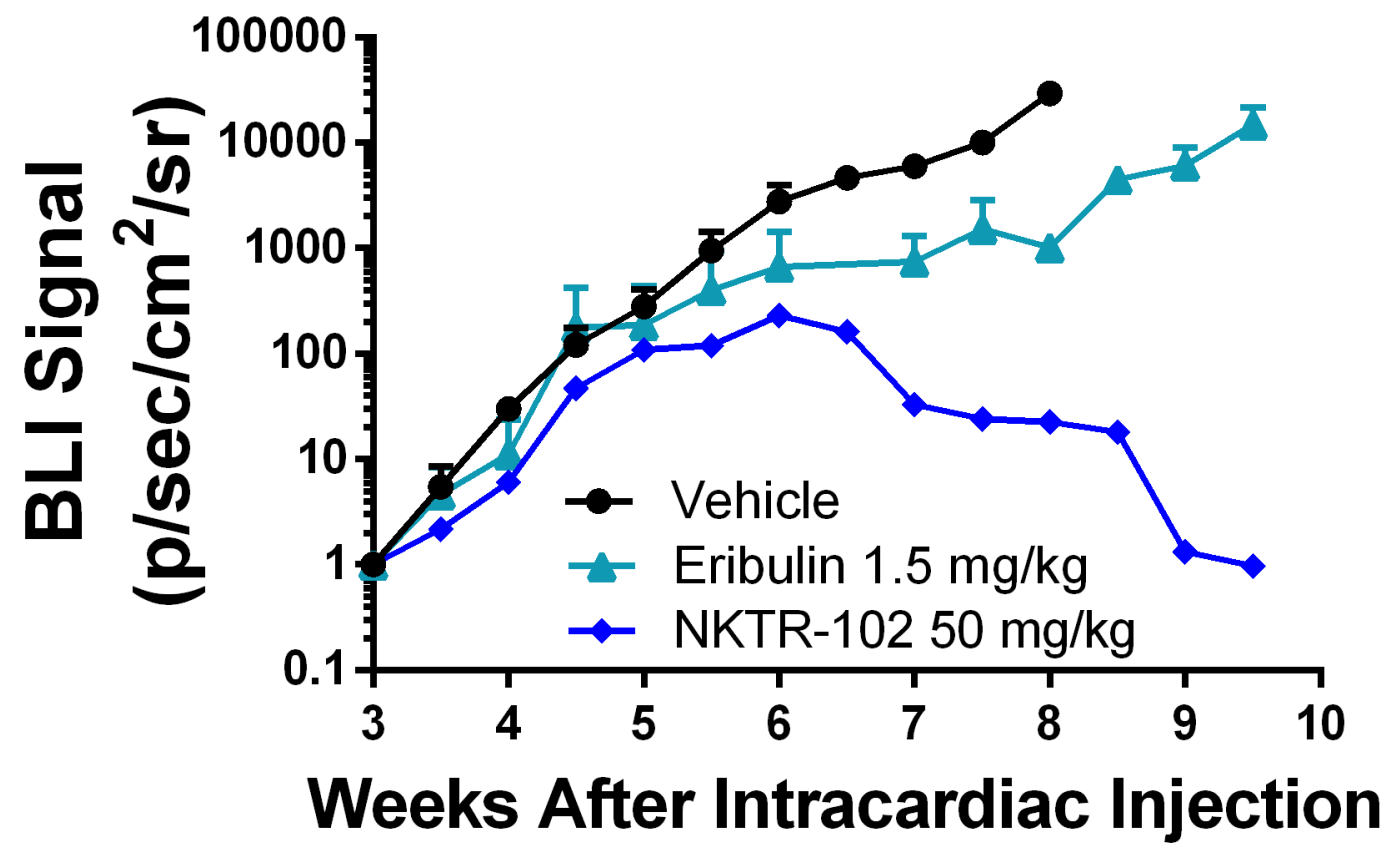

Supplemental Figure 6.1. Bioluminescence curves of conventional chemotherapy responders.

Two eribulin- and one NKTR-102-treated mice responded to therapy and showed a decrease in tumor burden. This did not impact the overall median survival of the groups. 


\begin{tabular}{|c|c|c|c|c|}
\hline Drug & Source & Dose & Formulation & Administration \\
\hline Afatinib & SelleckChem & $\begin{array}{l}30 \\
\mathrm{mg} / \mathrm{kg}\end{array}$ & $\begin{array}{l}5 \% \text { DMSO, } 10 \% \\
\text { polyethylene glycol 400, } \\
85 \% \text { distilled water }\end{array}$ & PO, every day \\
\hline Cisplatin & SelleckChem & $\begin{array}{l}5 \\
\mathrm{mg} / \mathrm{kg}\end{array}$ & $\begin{array}{l}5 \% \text { dimethylformamide, } \\
95 \% \text { saline }\end{array}$ & IV, every 7 days \\
\hline Docetaxel & SelleckChem & $\begin{array}{l}10 \\
\mathrm{mg} / \mathrm{kg}\end{array}$ & $\begin{array}{l}\text { 5\% Ethanol, 5\% Tween } \\
80,90 \% \text { saline }\end{array}$ & IV, every 7 days \\
\hline Doxil @ & $\begin{array}{l}\text { WVU } \\
\text { Pharmacy }\end{array}$ & $\begin{array}{l}6 \\
\mathrm{mg} / \mathrm{kg}\end{array}$ & $\begin{array}{l}\text { Pegylated liposomes in } \\
5 \% \text { dextrose }\end{array}$ & IV, every 7 days \\
\hline $\begin{array}{l}\text { Eribulin } \\
\text { mesylate }\end{array}$ & Adooq & $\begin{array}{l}1.5 \\
\mathrm{mg} / \mathrm{kg}\end{array}$ & $\begin{array}{l}5 \% \text { ethanol, } 15 \% \\
\text { distilled water, } 80 \% \\
\text { saline }\end{array}$ & SQ, every 4 days \\
\hline $\begin{array}{l}\text { Etirinotecan } \\
\text { pegol }\end{array}$ & $\begin{array}{l}\text { NEKTAR } \\
\text { Therapeutics }\end{array}$ & $\begin{array}{l}50 \\
\mathrm{mg} / \mathrm{kg}\end{array}$ & Saline & IV every 7 days \\
\hline Etoposide & SelleckChem & $\begin{array}{l}10 \\
\mathrm{mg} / \mathrm{kg}\end{array}$ & $\begin{array}{l}\text { 5\% DMSO, } 5 \% \text { Tween } \\
80,90 \% \text { Saline }\end{array}$ & $\begin{array}{l}\text { IV on days 2-5 } \\
\text { from cisplatin } \\
\text { administration }\end{array}$ \\
\hline Gefitinib & SelleckChem & $\begin{array}{l}6.25 \\
\mathrm{mg} / \mathrm{kg}\end{array}$ & $\begin{array}{l}5 \% \text { DMSO, } 10 \% \\
\text { polyethylene glycol 400, } \\
85 \% \text { distilled water }\end{array}$ & PO every day \\
\hline Gemcitabine & SelleckChem & $\begin{array}{l}60 \\
\mathrm{mg} / \mathrm{kg}\end{array}$ & Saline & SQ, every 4 days \\
\hline
\end{tabular}




\begin{tabular}{|c|c|c|c|c|}
\hline Irinotecan & $\begin{array}{l}\text { NEKTAR } \\
\text { Therapeutics }\end{array}$ & $\begin{array}{l}50 \\
\mathrm{mg} / \mathrm{kg}\end{array}$ & $5 \%$ Dextrose & IV, every 7 days \\
\hline Osimertinib & SelleckChem & $\begin{array}{l}25 \\
\mathrm{mg} / \mathrm{kg}\end{array}$ & $\begin{array}{l}5 \% \text { DMSO, } 10 \% \\
\text { polyethylene glycol } 400 \text {, } \\
85 \% \text { distilled water }\end{array}$ & PO, every day \\
\hline Pemetrexed & SelleckChem & $\begin{array}{l}100 \\
\mathrm{mg} / \mathrm{kg}\end{array}$ & Saline & $\begin{array}{l}\text { IV, on days } 3,4 \text {, } \\
5 \text { from cisplatin } \\
\text { administration }\end{array}$ \\
\hline Vinorelbine & SelleckChem & $\begin{array}{l}10 \\
\mathrm{mg} / \mathrm{kg}\end{array}$ & Saline & IV, every 7 days \\
\hline
\end{tabular}

Table 6.1. Formulations and dosing schedule for NKTR-102 in the PC-9-Br model. 


\begin{tabular}{|c|c|c|c|c|}
\hline Treatment & $\begin{array}{c}\text { Median Survival } \\
\text { (days) }\end{array}$ & $\mathbf{n}$ & $\begin{array}{c}\text { Surviving } \\
(\%)\end{array}$ & $\begin{array}{c}\text { vs. Vehicle (Log- } \\
\text { Rank) }\end{array}$ \\
\hline \multicolumn{5}{|c|}{ BEACON AGENTS } \\
\hline Vehicle & 42 & 10 & 0 & \\
\hline Irinotecan & 42 & 9 & 0 & 0.6720 \\
\hline NKTR-102 & 43 & 10 & $1(10 \%)$ & 0.1609 \\
\hline Gemcitabine & 45.5 & 10 & 0 & 0.1055 \\
\hline Docetaxel & 42.5 & 10 & 0 & 0.7636 \\
\hline Eribulin & 43.5 & 10 & $2(20 \%)$ & 0.1246 \\
\hline Vinorelbine & 41 & 9 & 0 & 0.2364 \\
\hline \multicolumn{5}{|c|}{ CONVENTIONAL LUNG CHEMOTHERAPY AGENTS } \\
\hline $\begin{array}{l}\text { Cisplatin + } \\
\text { Etoposide }\end{array}$ & 51.5 & 10 & 0 & $0.0331 *$ \\
\hline $\begin{array}{l}\text { Cisplatin + } \\
\text { Pemetrexed }\end{array}$ & 45 & 9 & 0 & 0.2626 \\
\hline \multicolumn{5}{|c|}{ EGFR-TYROSINE KINASE INHIBITOR AGENTS } \\
\hline Gefitinib & 48 & 10 & 0 & 0.1336 \\
\hline Afatinib & (73) & 9 & $8(89 \%)$ & $<0.001 * *$ \\
\hline Osimeritinib & (73) & 8 & $7(88 \%)$ & $<0.001 * *$ \\
\hline
\end{tabular}

Table 6.2. Survival data of NKTR-102 against chemotherapy in the PC-9-Br model.

Neither BEACON chemotherapy, NKTR-102, or cisplatin-pemetrexed improved median survival. 


\section{CHAPTER 7}

\section{PRELIMINARY DATA OF ETIRINOTECAN PEGOL (NKTR-102) EFFICACY IN A PRECLINICAL GLIOBLASTOMA MODEL}

\subsection{Introduction}

Primary brain malignancies make up a smaller but significant portion of brain tumors compared to brain metastases. Within primary brain tumors, almost $80 \%$ are gliomas, with glioblastoma representing the most aggressive, invasive, and fatal subtype. [1] On diagnosis, many patients have an advanced disease state featuring a large, heterogeneously distributed tumor on imaging. The symptoms and mortality of glioblastoma is due to mass effect and compression on tissues, such as the brainstem. [2]

Current treatment options for glioblastoma include either whole-brain or stereotactic radiation combined with surgical evacuation, with chemotherapy used to reduce tumor burden. [3] Temozolomide is the most utilized therapy in glioblastoma [4] and many chemotherapy trials use temozolomide as a backbone as combination therapy. Other therapy for glioblastoma include Gliadel@ (carmustine wafers) [5] and a new device Optune ${ }^{\circledR}$, a non-invasive tumor-treating field therapy. [6] Investigational therapy includes photodynamic therapy (NCT03048240), antibody therapy (NCT03139916), sonic hedgehog pathway inhibition (NCT03466450), and pegylated chemotherapy. [7]

Though glioblastoma is typically rapid-growing, the U251 model has exhibited more than a 50-day median survival in multiple models. [8, 9] As we have demonstrated earlier, a novel PEGylated form of irinotecan has been shown to work on the larger and slow-growing MDA-MB-231 triple-negative and HER2+ JIMT1 breast cancer brain 
metastases models (Chapter 4), but not the more rapidly growing and smaller 4T1 breast cancer or PC-9 lung cancer brain metastases models (Chapter 6). It is thought that NKTR-102 accumulates in tumors and provides sustained chemotherapy effects throughout its long half-life. [10]

Herein, we report the preliminary survival efficacy of NKTR-102 in the intracranial U251 glioblastoma model. A dose of $50 \mathrm{mg} / \mathrm{kg}$ NKTR-102 allowed 7 of 8 treated mice to survive until the end of our trial at 100 days, while vehicle-treated mice had a median survival of 73 days. Tumor burden was controlled and minimized within one week of NKTR-102 therapy initiation and remained below treatment initiation levels throughout the trial. This supports the use of NKTR-102 in the treatment of slow-growing glioblastomas.

\subsection{Methods and Materials}

Animals

The animal protocol used is the same as in Chapter 4.2.

\section{Cells}

The original U251 cell line was provided by Dr. Gordon Meares. A lentivirus containing Firefly luciferase (Fluc) with the hygromycin selection marker was purchased from Biosettia (GlowCell-16h). $10 \mu \mathrm{L}$ of viral vector and $10 \mu \mathrm{L}$ of polybrene (Santa Cruz, sc-134220) was added to each well in a 6-well plate of U251 cells at 70\% confluence. Plates were spun at 1000 RPM for 60 minutes and placed in a 37C incubator overnight. The following morning, cells were washed with phosphate-buffer saline (PBS) 
and then introduced to hygromycin at a concentration of $10 \mu \mathrm{L} / \mathrm{mL}$ in media to ensure successful transduction. After a week, selected cells checked for luciferase expression.

\section{Intracranial injection}

Mice were anesthetized under $2 \%$ isoflurane and placed in an intracranial stereotactic injection device (Stoelting). 500,000 U251 cells were suspended in PBS $(100,000$ per $1 \mu \mathrm{L})$ and injected over 5 minutes. $1 \mu \mathrm{L}$ was injected every 45 seconds for 4 $\mu \mathrm{L}$, after a minute pause to let cells distribute, the last $1 \mu \mathrm{L}$ was injected and the syringe was pulled out after another minute.

\section{Drugs and dosing}

NKTR-102 at $50 \mathrm{mg} / \mathrm{kg}$ equivalents of irinotecan (11 mg of NKTR-102 per mouse) was dissolved in PBS for a total of $200 \mu \mathrm{L} /$ mouse. PBS was used as the vehicle. Doses were administered weekly via tail vein to an anesthetized mouse, starting on Day 49.

\section{Bioluminescence imaging}

The bioluminescence imaging protocol used is the same from Chapter 5.2. The timing of injection is based on the pharmacokinetic profiling of Chapter 3.

In vitro toxicology

The toxicology protocol used is the same from Chapter 4.2.

\subsection{Results}

U251 glioblastoma cells are sensitive to $\mathrm{SN}-38$ in the nanomolar range 
When exposed to 1 femtomolar to 1 millimolar concentrations, the $\mathrm{IC}_{50}$ value of U251 cells to SN-38, the active metabolite of NKTR-102, was $0.536 \mathrm{nM}$ (Fig. 7.1). This is lower than in the MDA-MB-231-Br model (10.5 nM), 4T1-Br model (24.9 nM), JIMT1 model (222.8 nM) (Table 4.2), or PC-9-Br model (6.0 uM) (Fig. 6.1).

\section{NKTR-102 controls tumor burden in the preclinical U251 glioblastoma model}

Having seen that NKTR-102 improves survival in the preclinical breast-brain metastatic model due to accumulation in tumors, we then evaluated its efficacy in a glioblastoma model. U251 cells were intracranially injected and allowed to grow for 49 days (baseline), at which animals were randomized to either vehicle or NKTR-102. BLI was taken twice weekly to monitor tumor burden and chemotherapy response (Fig. 7.2). In vehicle animals, tumor burden increased 103-fold over baseline across 7 weeks, while in weekly-treated NKTR-102 $50 \mathrm{mg} / \mathrm{kg}$, tumor burden was controlled from initiation and reduced 5-fold over the same time span (Fig. 7.3A). Survival reflected BLI, as median survival in vehicle was 73 days, with all but one mouse administered with NKTR-102 surviving until the end of the 100-day trial (Fig. 7.3B). Of interest, NKTR-102 provided sustained tumor burden control but did not eliminate BLI signal in any mouse despite 7 weeks of dosing.

\subsection{Discussion}

As the U251 had the most sensitivity to SN-38 of all cell lines tested (Table 4.2,

Table 6.2, Fig. 7.1), it was hoped that NKTR-102 administration would lead to significant survival benefit and better outcomes than any previous model. This study shows that weekly NKTR-102 successfully controls tumor burden and improves survival in an intracranial glioblastoma model compared to vehicle therapy. 
The efficacy of NKTR-102 is now exhibited in three models, the first two being triple-negative and HER2+ BCBM. [10][Chapter 4] The reduction of tumor BLI signal occurred within one week of NKTR-102 dosing in the glioblastoma model, similar to the MDA-MB-231-Br BCBM model (Fig. 3.2), and stayed below baseline for the duration of treatment. The survival benefit was significant as NKTR-102 allowed 7 of 8 mice to survive until the end of therapy administration.

The vascularity within glioblastomas are extremely heterogeneous, with central cores possessing enhanced permeability while peripheral areas retaining intact permeability. [11, 12] This intra-lesion discordance may explain NKTR-102's ability to significantly reduce, but not completely eliminate tumor presence from intracranial lesions, even after weeks of dosing.

The basis for NKTR-102 efficacy relies on two factors: size and permeability. As a 20-kDa molecule, NKTR-102 preferentially accumulates into tumor tissue [10] (Fig. 4.7). Its large size allows the molecule to bypass efflux pumps such as P-glycoprotein; present in endothelial and astrocytic components of the BBB and BTB. Retention of the molecule within the circulatory system allows for a long half-life, and subsequent deposition and residence in tumor parenchyma is a process known as enhanced permeation and retention (EPR). [13]

The permeability of NKTR-102 through leaky vasculature plays a large role in its efficacy. [10] Through deposition into tumor tissue and continual release of irinotecan with subsequent metabolism to $\mathrm{SN}-38$, NKTR-102 provides substantial and sustained cytotoxic effects to reduce tumor burden. The additional advantage of a long circulating 
half-life provided by pegylation allows for less frequent dosing, which provides another advantage over conventional irinotecan dosing. [10]

In conjunction with EPR, it is possible that NKTR-102 finds its efficacy in slowgrowing and larger tumors. In the preclinical BCBM models, the 231-Br and HER2+ are noted to have larger tumors while 4T1 are more numerous, small, and grow relatively rapidly. [14] While NKTR-102 improved median survival in the 231-Br and HER2+ lines, it did not do so in the 4T1 line (Chapter 4). Similarly, the PC-9-Br line creates smaller and rapidly-growing tumors; NKTR-102 administration did not improve median survival compared to vehicle in this cell line (Chapter 6). Though tumor size and permeability are not related, it is known that glioblastoma form larger tumors and are highly permeable compared to metastatic models. [14, 15] In the U251 model, NKTR102 may create a depot and provide sustained tumor burden control, though the cellular growth may outpace the delivery of drug as evidenced by bioluminescence data.

With NKTR-102 involved in multiple clinical trials ranging from treatment of metastatic breast cancer, including the ATTAIN trial focusing on BCBM, these preliminary results show that NKTR-102 causes rapid control of tumor burden from primary intracranial lesions with significant survival benefits.

\subsection{Conclusion}

NKTR-102 controls tumor burden and significantly extends survival compared to vehicle in the preclinical intracranial U251 glioblastoma model. The next step is to test conventional irinotecan and glioblastoma therapy (temozolomide) against NKTR-102 to determine if it improves survival compared to currently chemotherapeutics. 


\subsection{References}

1. Goodenberger, M.L. and R.B. Jenkins, Genetics of adult glioma. Cancer Genet, 2012. 205(12): p. 613-21.

2. Hanif, F., et al., Glioblastoma Multiforme: A Review of its Epidemiology and Pathogenesis through Clinical Presentation and Treatment. Asian Pac J Cancer Prev, 2017. 18(1): p. 3-9.

3. Coleman, N., M. Ameratunga, and J. Lopez, Development of Molecularly Targeted Agents and Immunotherapies in Glioblastoma: A Personalized Approach. Clin Med Insights Oncol, 2018. 12: p. 1179554918759079.

4. Davis, M.E., Glioblastoma: Overview of Disease and Treatment. Clin J Oncol Nurs, 2016. 20 (5 Suppl): p. S2-8.

5. Chowdhary, S.A., T. Ryken, and H.B. Newton, Survival outcomes and safety of carmustine wafers in the treatment of high-grade gliomas: a meta-analysis. $\mathrm{J}$ Neurooncol, 2015. 122(2): p. 367-82.

6. Mehta, M., et al., Critical review of the addition of tumor treating fields (TTFields) to the existing standard of care for newly diagnosed glioblastoma patients. Crit Rev Oncol Hematol, 2017. 111: p. 60-65.

7. Nagpal, S., et al., Phase II pilot study of single-agent etirinotecan pegol (NKTR102) in bevacizumab-resistant high grade glioma. J Neurooncol, 2015. 123(2): p. $277-82$.

8. Day, B.W., et al., EphA3 maintains tumorigenicity and is a therapeutic target in glioblastoma multiforme. Cancer Cell, 2013. 23(2): p. 238-48. 
9. Ke, L.D., Y.X. Shi, and W.K. Yung, VEGF(121), VEGF(165) overexpression enhances tumorigenicity in U251 MG but not in NG-1 glioma cells. Cancer Res, 2002. 62(6): p. 1854-61.

10. Adkins, C.E., et al., NKTR-102 Efficacy versus irinotecan in a mouse model of brain metastases of breast cancer. BMC Cancer, 2015. 15: p. 685.

11. Zhang, F., C.L. Xu, and C.M. Liu, Drug delivery strategies to enhance the permeability of the blood-brain barrier for treatment of glioma. Drug Des Devel Ther, 2015. 9: p. 2089-100.

12. van Tellingen, O., et al., Overcoming the blood-brain tumor barrier for effective glioblastoma treatment. Drug Resist Updat, 2015. 19: p. 1-12.

13. Hoch, U., et al., Nonclinical pharmacokinetics and activity of etirinotecan pegol (NKTR-102), a long-acting topoisomerase 1 inhibitor, in multiple cancer models. Cancer Chemother Pharmacol, 2014. 74(6): p. 1125-37.

14. Adkins, C.E., et al., Characterization of passive permeability at the blood-tumor barrier in five preclinical models of brain metastases of breast cancer. Clin Exp Metastasis, 2016. 33(4): p. 373-83.

15. Mittapalli, R.K., et al., Quantitative Fluorescence Microscopy Measures Vascular Pore Size in Primary and Metastatic Brain Tumors. Cancer Res, 2017. 77(2): p. 238-246. 


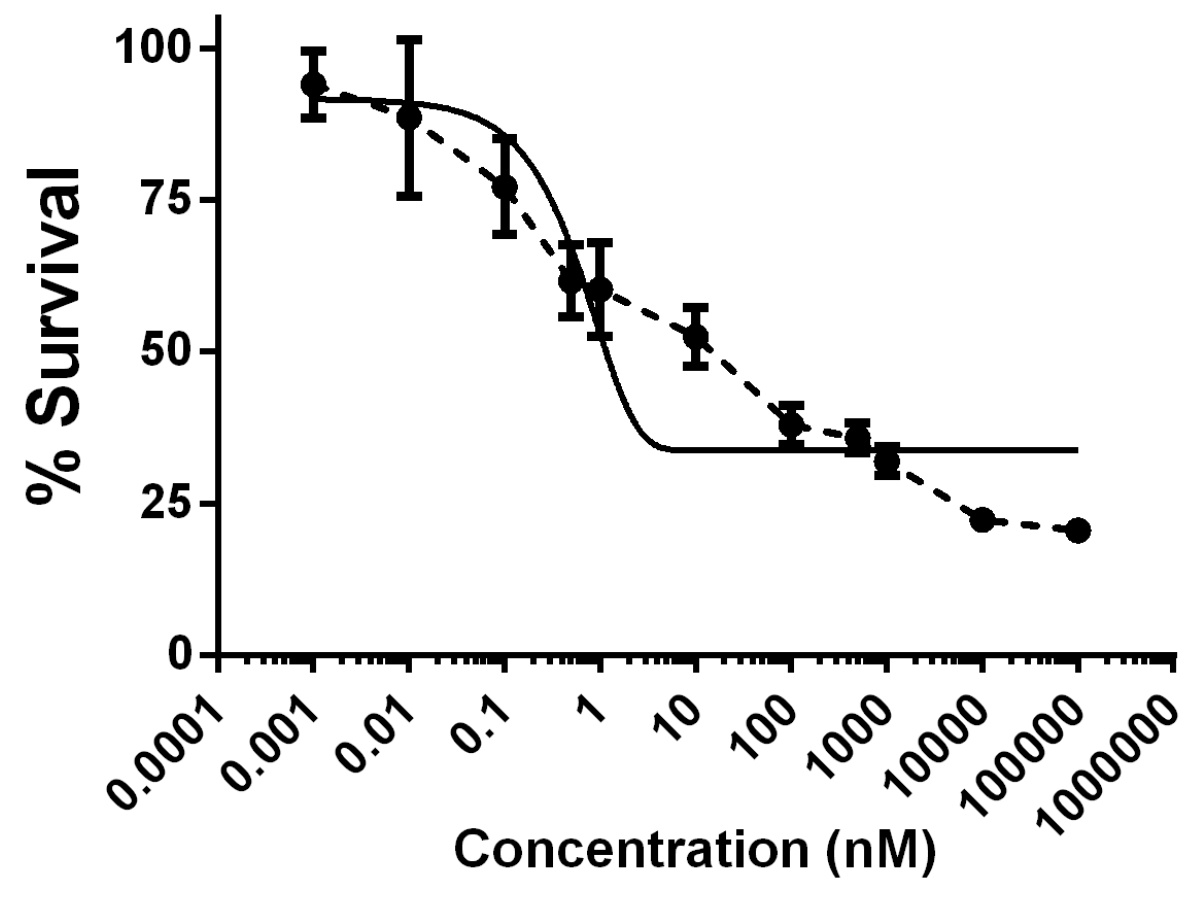

Figure 7.1. $\mathrm{U} 251$ is sensitive to $\mathrm{SN}-38$ in the nanomolar range.

The dashed line shows a linearity in dose reduction with log-increases of SN-38, while the solid line is the non-linear best-fit curve. The U251 glioblastoma cell line is sensitive to $\mathrm{SN}-38$ at $0.6 \mathrm{nM}$. 


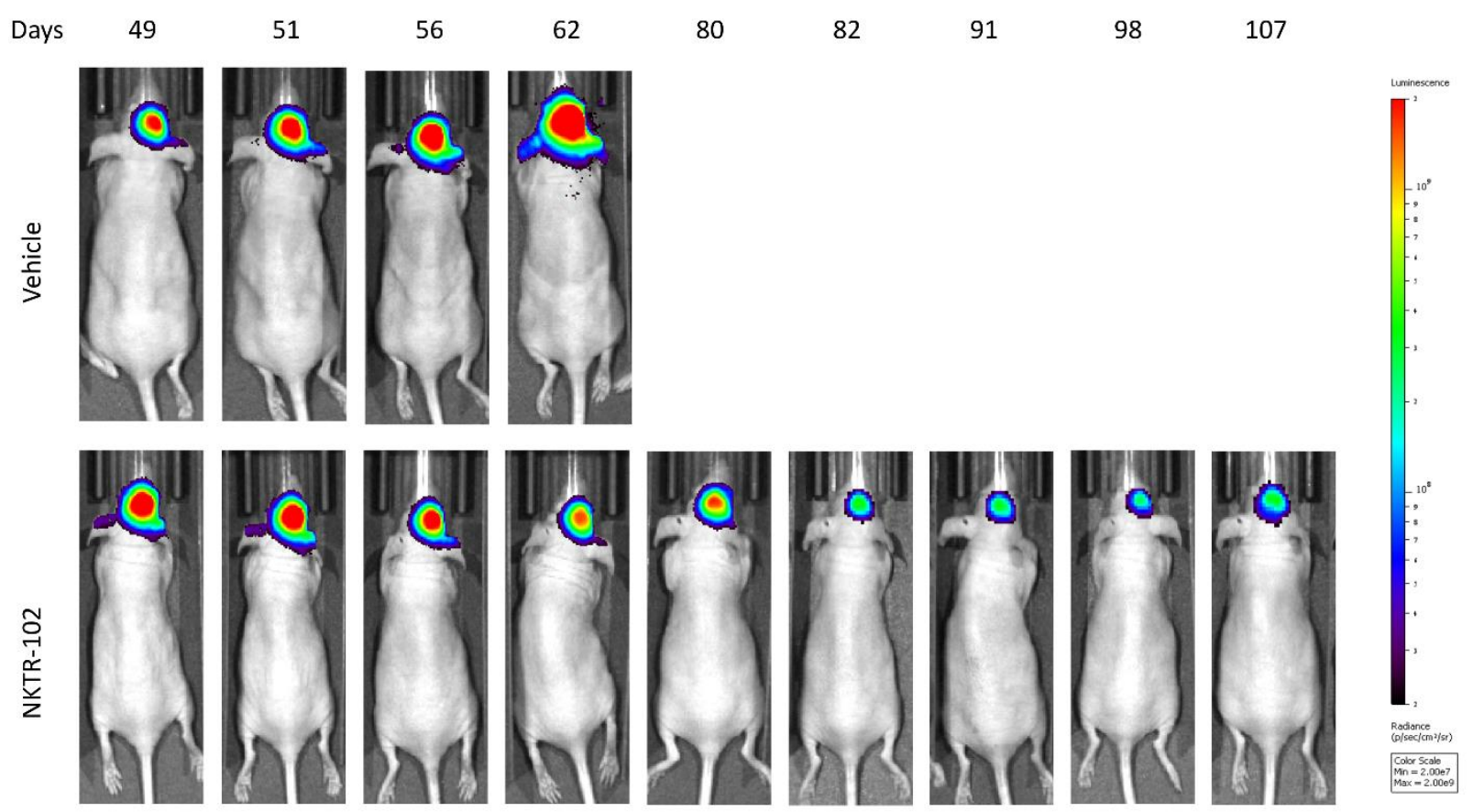

Figure 7.2. NKTR-102 enhances survival compared to vehicle therapy in the U251 model.

Following intracranial injection of U251 glioblastoma cells and establishment of brain metastases 49 days later, biweekly imaging on the IVIS Spectrum CT tracked tumor growth and etirinotecan pegol chemotherapeutic efficacy. Images shown are of the same animal sequentially. While vehicle bioluminescence increases until animal death, etirinotecan pegol controls and lowers tumor burden until the end of trial. 


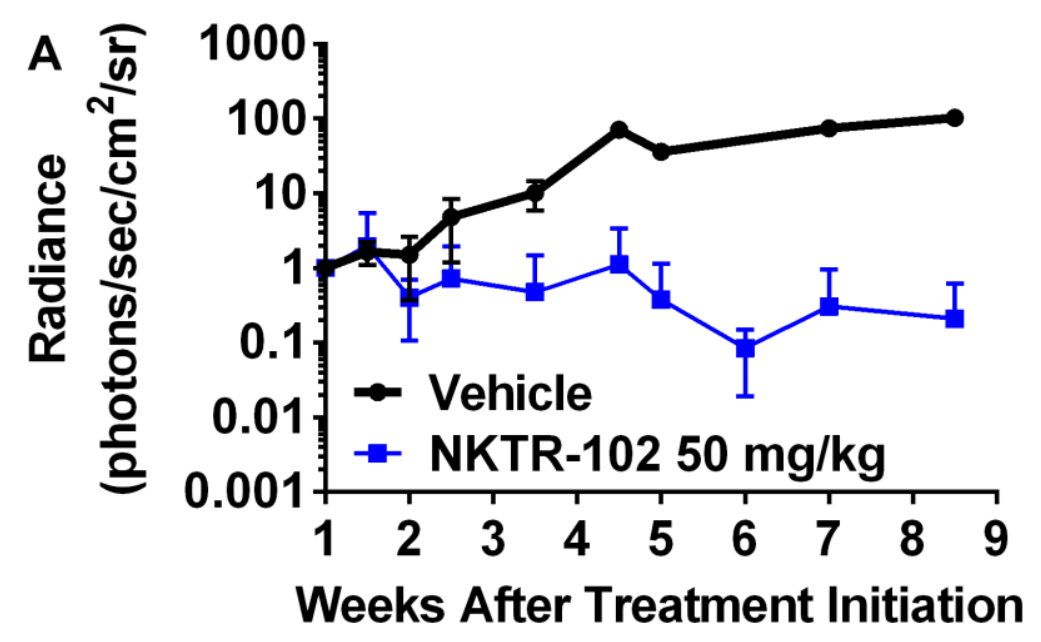

B

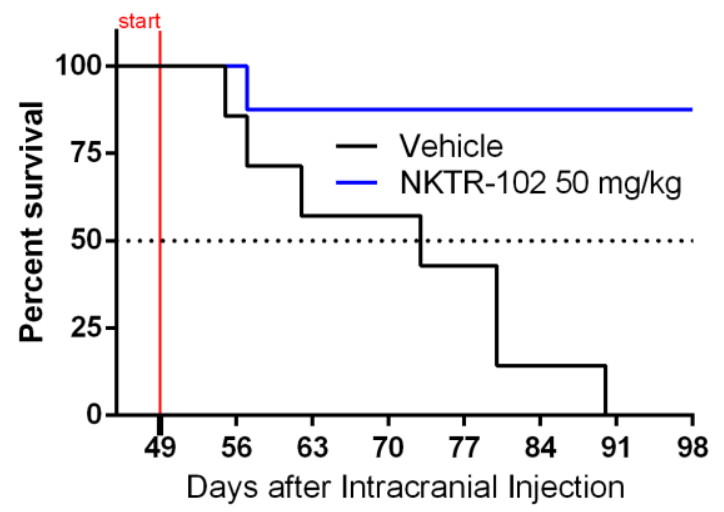

Figure 7.3. Etirinotecan pegol reduces tumor burden and improves survival compared to vehicle in the $\mathbf{U} 251$ glioblastoma model.

(A) Mean BLI signal versus time in mice treated with therapy. Treatment was initiated on day 49. Each data point represents mean $\pm \operatorname{SD}$ ( $n=2-10$ per time point). Tumor burden in etirinotecan pegol-treated mice was significantly lower than conventional chemotherapy or vehicle treatment groups $(\mathrm{p}<0.05)$. (B) Etirinotecan pegol significantly increased median survival in tumor mice compared to vehicle, with 9 mice treated with etirinotecan surving until the end of the trial $(\mathrm{p}>0.05)$. 


\section{CHAPTER 8}

\section{PRELIMINARY DATA OF ABRAXANE AND DOXIL EFFICACY IN PRECLINICAL BRAIN METASTASES MODELS}

\subsection{Introduction}

Metastases remains one of the leading causes of death from cancer. [1] The most common organs cancers metastasize to are summed in the mnemonic "BBBLL": breast, bone, brain, liver, and lung. [2] Of these sites, metastases to the brain is the most difficult to treat due to the sanctuary state provided by the blood-brain and blood-tumor barriers, as well as the difficulty in performing surgical excavations in the small space of a critical organ. Though advances have been made in surgery and radiation therapy, significant advances have been made in the realm of chemotherapy to treat brain metastases.

Two current trends exist: to develop new molecules, and to repurpose existing molecules into novel dosage forms. Four common repurposing techniques involve liposome encapsulation, PEGylation, nanoparticle creation, and antibody conjugation. [3] These are often constrained by size to avoid rapid kidney elimination and destruction by macrophages. [3] Novel dosage forms may also reduce total amounts of drug required to achieve a therapeutic effect, reduce side effects from dosing, and provide sustained release of chemotherapy over time. [3] Examples of novel chemotherapeutic agents being explored to treat brain metastases include NKTR-102 (etirinotecan pegol), T-DM1 (trastuzumab emtansine), Abraxane (nano-albumin-bound paclitaxel), and Doxil (pegylated liposomal doxorubicin). 
Abraxane, a 130-nanometer nanoparticle albumin-bound conjugate of paclitaxel, (nab-paclitaxel), is FDA-approved for metastatic breast cancer, non-small cell lung cancer, and pancreatic cancer. $[4,5]$ Compared to conventional paclitaxel, nab-paclitaxel displays a higher amount of systemic drug exposure, faster possible infusion rates, and higher unbound drug due to not requiring Cremophor EL as a cosolvent, slower drug elimination, and reduced drug toxicity. [6-8] Its longer half-life may be due to its formulation with albumin, which allows albumin-mediated transport of paclitaxel through vasculature and into tumor parenchyma. [8]

Doxil, a pegylated liposomal formulation of doxorubicin, is FDA-approved for ovarian cancer and multiple myeloma. [9] Pegylation of liposomes allows reduced removal through the spleen and liver, and additionally reduces the amount of drug escaping through liposomes and subsequent elimination of free drug. [10] By encapsulating doxorubicin into pegylated liposomes, the total amount of drug delivered (AUC) and half-life increases almost 30-fold, while clearance drops almost 100-fold, when compared to conventional doxorubicin. [10]

Herein, we show that though the triple-negative breast cancer MDA-MB-231-Br cells are more sensitive to Abraxane than Doxil in-vitro, only Doxil significantly improves survival in tumor-bearing mice. Doxil does not improve survival in the EGFRmutated PC-9 preclinical LCBM model, which corresponds with other studies showing only targeted tyrosine kinases improve survival in this setting.

\subsection{Methods and Materials}

\section{Animals}


The animal protocol used is the same as in Chapter 4.2.

\section{Cells}

The MDA-MB-231-Br and PC-9-Br cells used in this Chapter are the same as in Chapter 4 and Chapter 5, respectively.

\section{Tumor cell injection}

The intracardiac injection protocol used is the same as in Chapter 4.2.

\section{Drugs and dosing}

Abraxane was provided as a 1-gram powder of $100 \mathrm{mg}$ paclitaxel to $900 \mathrm{mg}$ human albumin. Doses were based on amount of paclitaxel. Weighed powder was then suspended in phosphate buffer saline to create a suspension. $50 \mathrm{mg} / \mathrm{kg}$ was given weekly as an intravenous dose, then $25 \mathrm{mg} / \mathrm{kg}$ via intraperitoneal injection 3 to 4 days later. Doxil came solubilized at $2 \mathrm{mg}$ per $\mathrm{mL}$ and was further diluted in dextrose $5 \%$ prior to administration at a weekly dose of $6 \mathrm{mg} / \mathrm{kg}$ intravenously. [11]

\section{Statistics}

Survival data were compared using the log-rank method. Significance is defined as $\mathrm{p}<0.05$. All statistics were performed on GraphPad Prism 6.

\section{Bioluminescence imaging}

The bioluminescence imaging protocol used is the same from Chapter 4.2.

\section{In vitro toxicology}

The toxicology protocol used is the same from Chapter 4.2. 


\subsection{Results}

\section{1-Br cells are more sensitive to Abraxane than Doxil}

To test sensitivity of the triple-negative BCBM line 231, the MTT toxicology assay was used. Abraxane was suspended initially in PBS and diluted in media. Doxil, already provided in solution, was directly added to media and then diluted. The $\mathrm{IC}_{50}$ of 231-Br to Abraxane is $20.2 \mathrm{nM}$ (Fig. 8.1A), while the IC50 of 231-Br to Doxil is 2.93 $\mu \mathrm{M}$ (Fig. 8.1B).

Abraxane improves survival in the preclinical triple-negative breast-brain metastatic model

Abraxane was suspended in PBS and injected via tail vein of mice bearing 231-Br metastases 14 days after intracardic cancer cell injection. The median survival was 34.5 days, while in historical vehicle it was 36.5 days (Fig. 8.2). One mouse in the Abraxane group survived until 70 days, whereas no vehicle mice survived until day 50.

Doxil significantly improves survival over vehicle in the 231-Br model

Weekly intravenous injections of Doxil starting 14 days post-cancer cell injection led to a 670-fold increase in BLI, compared to the nearly 2000-fold increase from baseline seen in vehicle mice (Fig. 8.3A). The rate of BLI over time is similar to that of vehicle until week 7 of dosing, where the curve represents one surviving mouse. Doxil administration improved median survival to of the 231-Br mice to 45 days, 9 more than vehicle $(\mathrm{p}<0.05)$ (Fig. 8.3B).

Doxil does not significantly control tumor growth in the PC-9-Br model 
As Doxil proved effective in the breast-brain metastatic model, interest turned to testing its survival efficacy in the PC-9 lung-brain metastatic model. 21 days after intracardiac injection with PC-9 cells, Doxil was intravenously injected at $6 \mathrm{mg} / \mathrm{kg}$ weekly. Though the BLI rose to only approximately 4500-fold above baseline compared to 29000 -fold in the vehicle group (Fig. 8.4A), survival was unaffected as both vehicle and Doxil-treated groups had a median survival of 42 days (Fig. 8.4B).

\subsection{Discussion}

This is the first study to evaluate survival effects of Abraxane (nano-albuminbound paclitaxel) and Doxil (pegylated liposomal doxorubicin) in the setting of brain metastases from breast cancer and lung cancer. Our finding for Doxil replicates another BCBM study in which survival was improved by 6 days compared to vehicle, though this study used the intracranial model of 231-Br. [11] In the clinical setting, Doxil has seen efficacy when combined with cyclophosphamide and temozolomide in the settings of brain metastases of solid tumors, including breast cancer.

The in vitro sensitivity of 231-Br to Abraxane is approximately 10-fold lower than sensitivity to Doxil, but this does not extrapolate to in vivo effects as survival is improved only in the Doxil-treated model. It is possible that albumin-bound drugs are unable to escape vasculature as efficiently as liposomes, as protein-binding reduces the ability of chemotherapy to penetrate through tight junctions. [12] It was determined that the pegylated liposomes of Doxil allow for extravasation through leaky tumor vasculature which extended circulation time and enhances drug delivery. [10] In the 231-Br model, vasculature is highly heterogenous and tumors are more permeable to smaller $(<200 \mathrm{Da})$ molecules than larger ones (> $500 \mathrm{Da})$. [13] This may explain our in vivo finding of a 
chemotherapy nanoparticle being more effective than a albumin-bound chemotherapeutic agent, though NKTR-102, a 20-kDa pegylated molecule, has improved survival in this same model and was shown to preferentially accumulate inside tumors. [14]

The lack of survival improvement with Doxil administration in the PC-9 lungbrain metastatic model is expected, as both conventional chemotherapy and NKTR-102, a pegylated form of irinotecan, similarly did not improve survival (Chapter 6). The tumor size and permeability characteristics of PC-9-Br are different than that of 231-Br: tumors are smaller and moderately permeable to ${ }^{14} \mathrm{C}$-AIB (Chapter 5). This again may explain why a $20-\mathrm{kDa}$ substance did not show survival advantage compared to targeted small molecule inhibitors, but does not explain why a 130-nm pegylated liposome failed to do so.

\subsection{Conclusion}

Abraxane and Doxil are novel formulations of existing drugs: paclitaxel and doxorubicin, respectively. While Abraxane is more potent in-vitro, Doxil controls tumor burden and significantly improves survival in the preclinical triple-negative MDA-MB231 breast cancer brain metastatic model. Doxil does not control tumor burden or significantly improve survival in the PC-9 lung cancer brain metastasis model. These agents should be tested in other metastatic models against other upcoming and approved chemotherapy. 


\subsection{References}

1. Seyfried, T.N. and L.C. Huysentruyt, On the origin of cancer metastasis. Crit Rev Oncog, 2013. 18(1-2): p. 43-73.

2. National Cancer Institute, N.C.I., Metastatic Cancer.

3. Ordikhani, F., et al., Drug Delivery Approaches for the Treatment of Cervical Cancer. Pharmaceutics, 2016. 8(3).

4. Ma, P. and R.J. Mumper, Paclitaxel Nano-Delivery Systems: A Comprehensive Review. J Nanomed Nanotechnol, 2013. 4(2): p. 1000164.

5. Kim, G., nab-Paclitaxel for the treatment of pancreatic cancer. Cancer Manag Res, 2017. 9: p. 85-96.

6. Chen, N., et al., Pharmacokinetics and pharmacodynamics of nab-paclitaxel in patients with solid tumors: disposition kinetics and pharmacology distinct from solvent-based paclitaxel. J Clin Pharmacol, 2014. 54(10): p. 1097-107.

7. Gardner, E.R., et al., Randomized crossover pharmacokinetic study of solventbased paclitaxel and nab-paclitaxel. Clin Cancer Res, 2008. 14(13): p. 4200-5.

8. Di Costanzo, F., et al., Targeted delivery of albumin bound paclitaxel in the treatment of advanced breast cancer. Onco Targets Ther, 2009. 2: p. 179-88.

9. Barenholz, Y., Doxil(R)--the first FDA-approved nano-drug: lessons learned. J Control Release, 2012. 160(2): p. 117-34.

10. Gabizon, A., H. Shmeeda, and Y. Barenholz, Pharmacokinetics of pegylated liposomal Doxorubicin: review of animal and human studies. Clin Pharmacokinet, 2003. 42(5): p. 419-36. 
11. Anders, C.K., et al., Pharmacokinetics and efficacy of PEGylated liposomal doxorubicin in an intracranial model of breast cancer. PLoS One, 2013. 8(5): p. e61359.

12. Owen, S. and L. Souhami, The management of brain metastases in non-small cell lung cancer. Front Oncol, 2014. 4: p. 248.

13. Adkins, C.E., et al., Characterization of passive permeability at the blood-tumor barrier in five preclinical models of brain metastases of breast cancer. Clin Exp Metastasis, 2016. 33(4): p. 373-83.

14. Adkins, C.E., et al., NKTR-102 Efficacy versus irinotecan in a mouse model of brain metastases of breast cancer. BMC Cancer, 2015. 15: p. 685. 

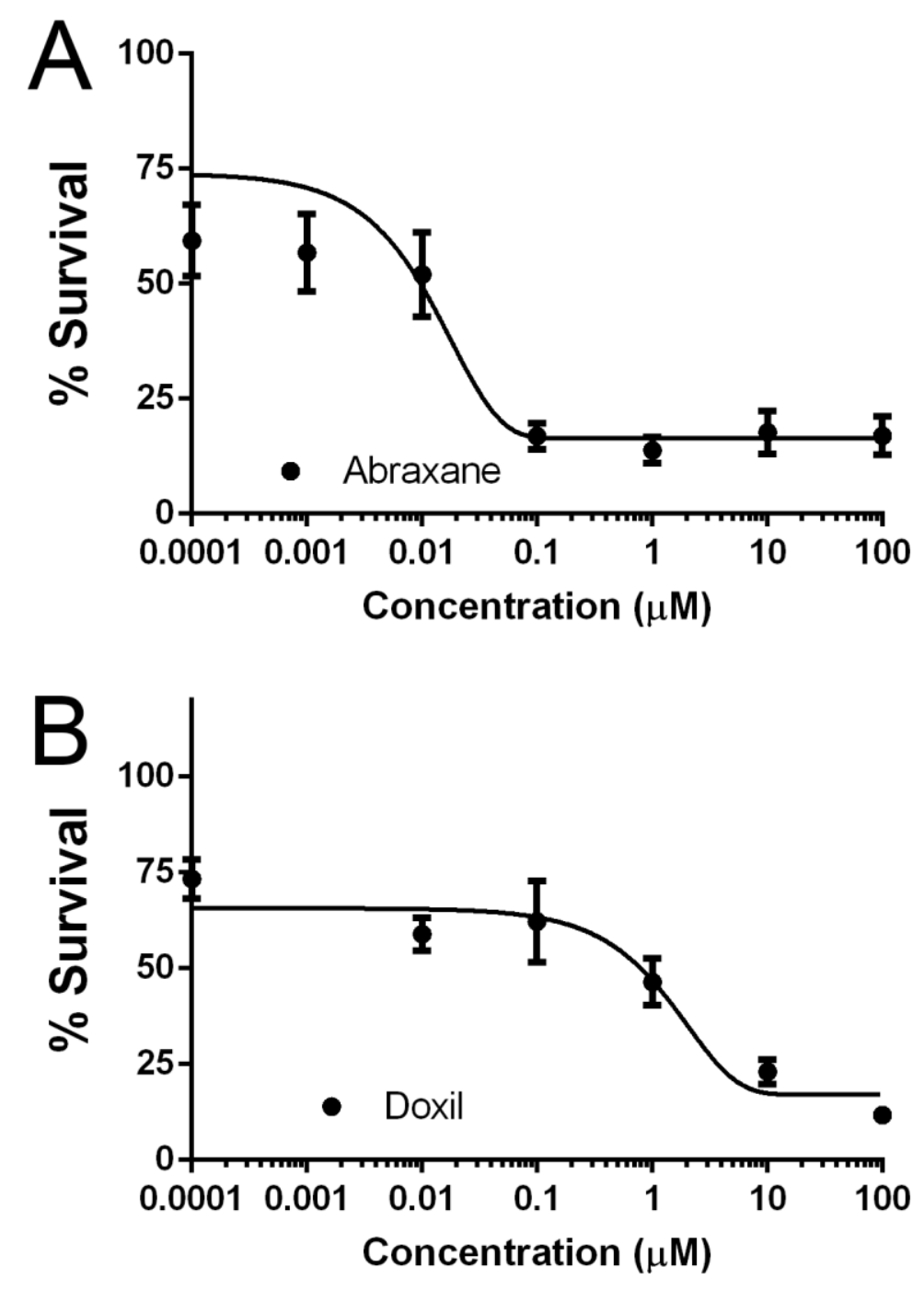

Figure 8.1. In-vitro cytotoxicity of Abraxane and Doxil on MDA-MB-231-Br cells.

(A) Cells are more sensitive to Abraxane than to (B) Doxil, though this correlation is not seen in-vivo. 


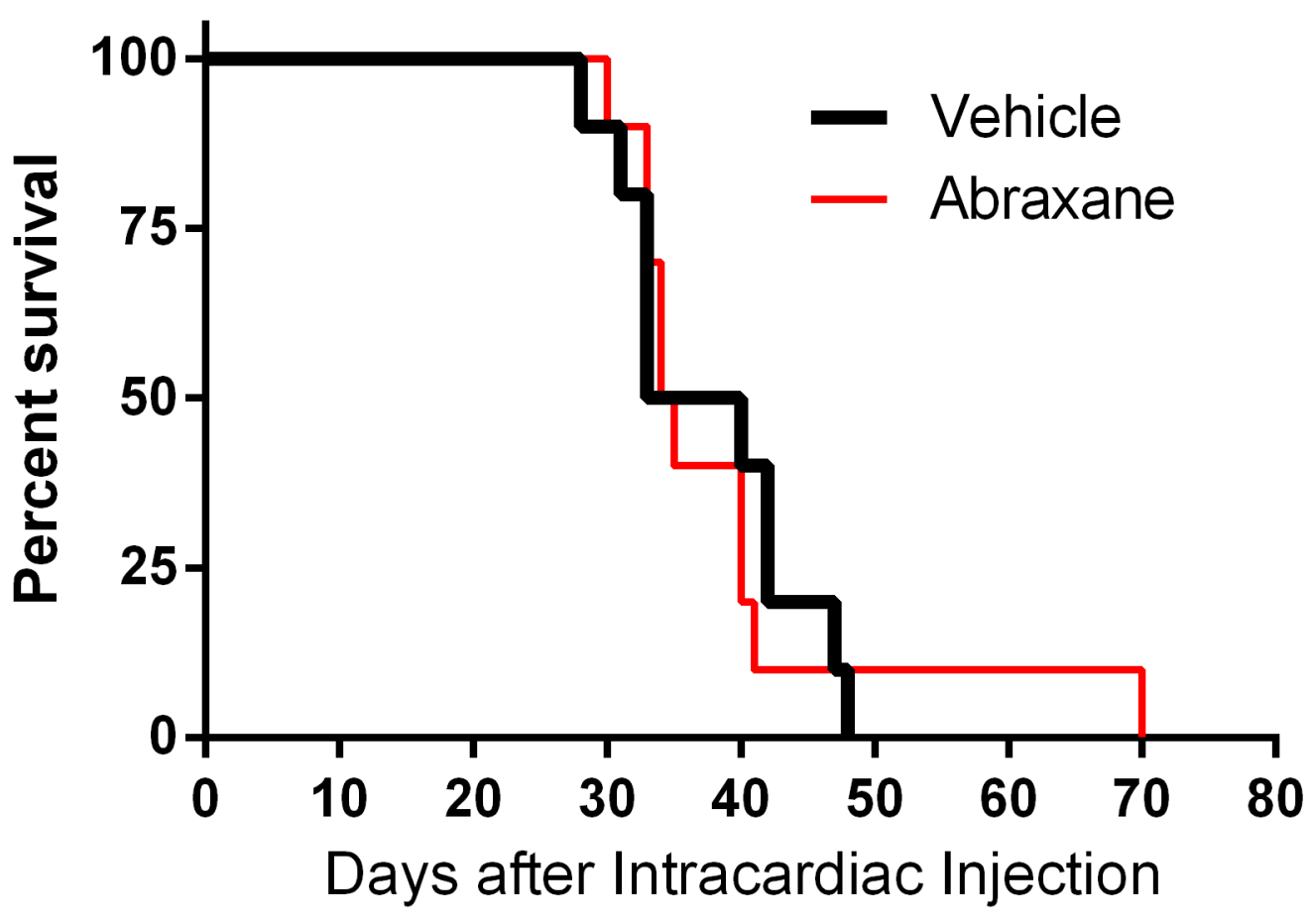

Figure 8.2. MDA-MB-231-Br survival is not improved on Abraxane therapy.

The median survival for vehicle is not significantly different from Abraxane therapy. 


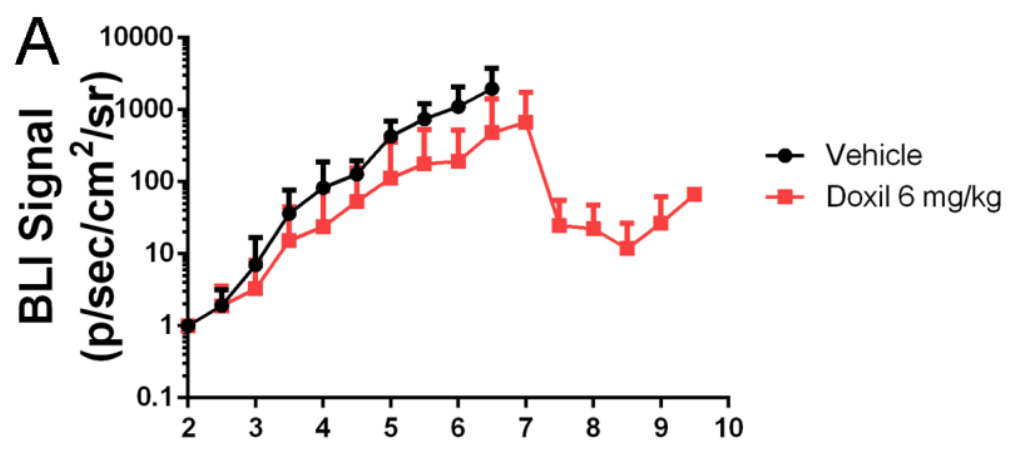

Weeks After Intracardiac Injection

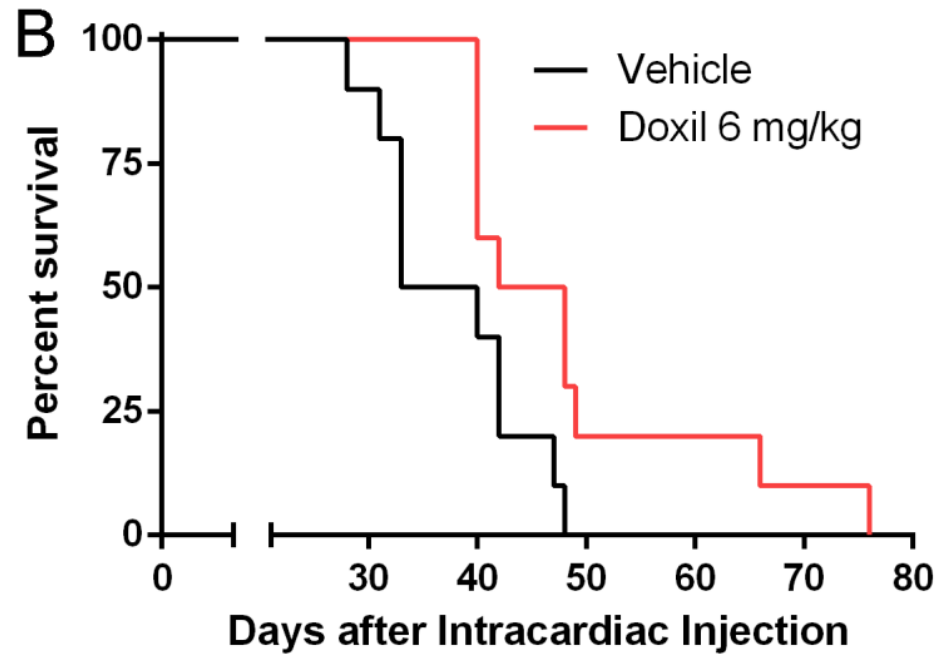

Figure 8.3. Doxil bioluminescence and survival effects on MDA-MB-231-Br tumors.

(A) Bioluminescence in Doxil-treated mice followed a similar albeit lower trend than vehicle, which dropped due to surviving mice. (B) Median survival was significantly improved in Doxil-treated mice using log-rank statistics. 

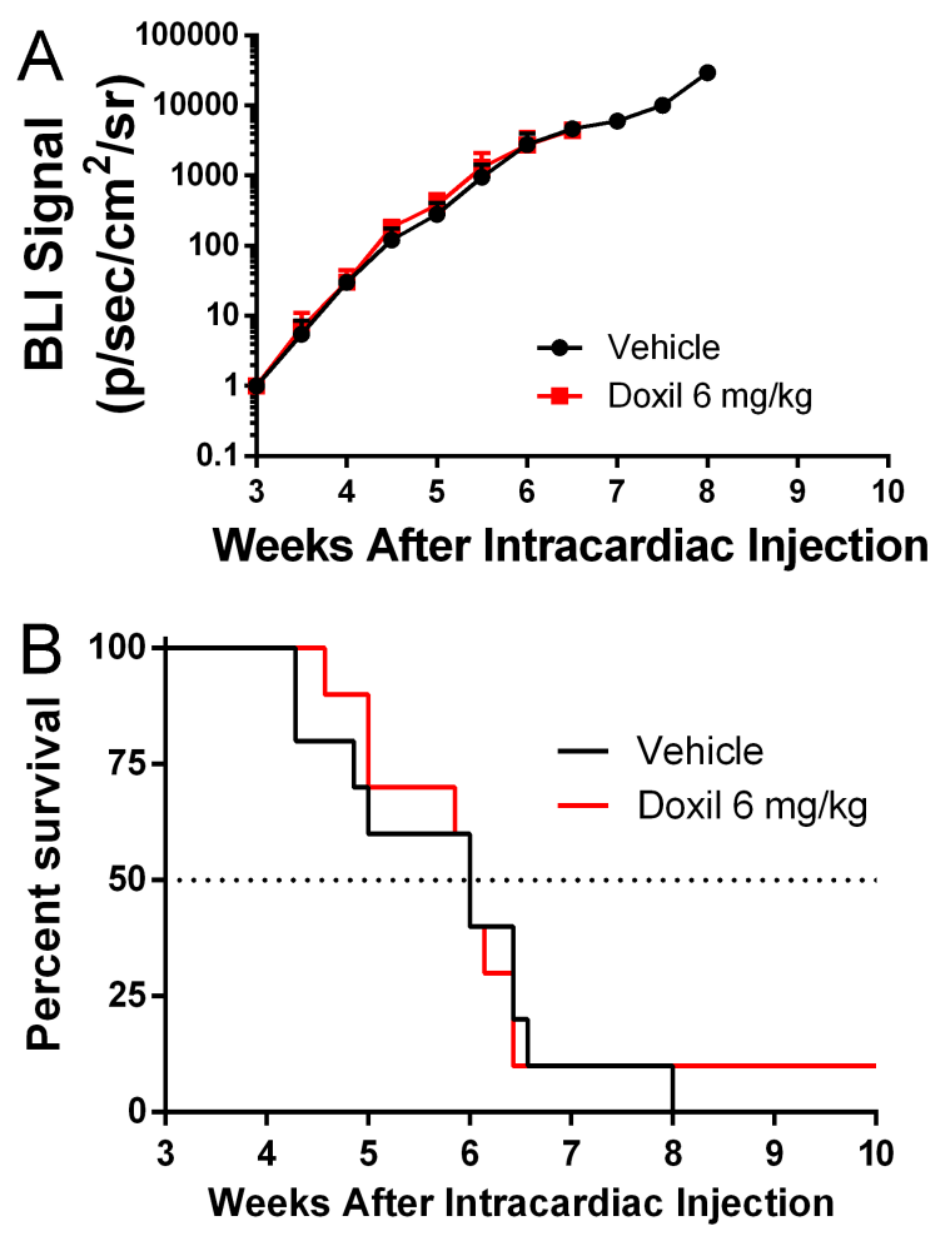

Figure 8.4. Effects of Doxil on the PC-9-Br model.

(A) Bioluminescence in Doxil-treated mice was not significantly different from vehicletreated mice (B) Median survival was not significantly improved in Doxil-treated mice using log-rank statistics. 


\section{CHAPTER 9}

\section{CONCLUSION AND FUTURE DIRECTIONS}

\subsection{Conclusion}

In conclusion, this dissertation (a) detailed the optimal imaging times and quantifiable intervals of the non-toxic tumor surrogate marker D-luciferin in metastatic and primary brain tumors, (b) characterized the creation, passive permeability, efflux pump status, and vascularity of a widely-used EGFR-mutant lung cancer cell line, (c) evaluated the in-vitro cytotoxicity and in-vivo efficacy of three novel chemotherapeutic agents on brain metastases of lung and breast cancer as well as primary glioblastoma, and (d) provided a review of the current clinical and preclinical treatment options for breast cancer and lung cancer brain metastases, and primary glioblastoma.

In order to evaluate the effects of tumor growth and response to chemotherapy, transfection of cancer cells with firefly luciferase prior to administration allows for a noninvasive, rapid, and quantifiable surrogate of tumor burden in a process known as

bioluminescence. The amount of light produced by cancer cells depends on a multitude of factors including method of cancer cell and/or D-luciferin injection, and possibly the amount of luciferase transfected in each cell. We found that lung-brain metastases had a faster onset to peak bioluminescence and a longer duration of signal, but shorter optimal (time $>95 \%$ peak luminescence time) compared to two models of breast-brain metastases; these breast-brain models were similar to each other in these parameters, indicating possible differences due to tissue origin. For intracranially injected glioblastoma cells, time-to-peak was even faster and optimal imaging time even shorter than lung-brain metastases, and produced more photons of light in a given time frame 
compared to the three metastatic models. In-vitro bioluminescence correlated nearly exactly with in-vivo findings, indicating that same timing and conditions allow for streamlining preclinical compound screening. With these results, we are able to determine the optimal D-luciferin pharmacokinetics for our longitudinal trials involving chemotherapy.

After determining luciferin pharmacokinetics in clinically relevant models, we sought to determine the efficacy of a novel chemotherapeutic agent compared to conventional chemotherapy utilizing bioluminescence as a surrogate for tumor burden. In the MDA-MB-231 triple-negative breast cancer brain metastases model, the pegylated form of irinotecan (etirinotecan pegol) was dosed at $50 \mathrm{mg} / \mathrm{kg}$ equivalents of irinotecan compared to gemcitabine, docetaxel, vinorelbine, and eribulin components of BEACON therapy. Etirinotecan pegol increased median survival compared to vehicle and BEACON agents, with four of ten mice surviving until the end of the trial. In the JIMT-1 HER2+ model, etirinotecan modestly improved survival compared to lapatinib and vehicle, and one mouse survived until the end of the trial. Bioluminescence showed increasing tumor burden in all conventional chemotherapy treated mice, with a plateau in etirinotecan pegol groups, signifying control of tumor burden. In contrast, the murine 4T1 model responded poorly to etirinotecan pegol, with only gemcitabine showing an improvement in median survival and tumor burden control compared to vehicle.

In the PC-9 lung cancer brain metastatic model, neither etirinotecan pegol nor any non-targeted conventional chemotherapy improved median survival or featured a bioluminescence curve that was significantly different from vehicle therapy. In contrast, two targeted EGFR-inhibitors showed early tumor burden control which was sustained 
until the end of the trial, with only one mouse dying of drug administration in each group. It is possible that the third targeted agent would improve survival if used at a higher dose. A preliminary intracranial U251 glioblastoma model revealed etirinotecan pegol having a similar early tumor burden control and improvement in survival compared to the vehicle group, with only one mouse dying until the end of trial. These findings pose the hypothesis that etirinotecan pegol may work best in slower growing and larger brain tumors.

While the breast-brain metastatic models and glioblastoma cell line have been extensively characterized, sufficient data does not exist for lung-brain metastases. Taken from terminally ill animals in the aforementioned study, a passive permeability marker $\left({ }^{14} \mathrm{C}\right.$-AIB), vascularity marker (ICG), and P-glycoprotein marker (OG) were used to determine characteristics of the PC-9 brain metastatic model. Fold increases compared to non-tumor regions were analyzed. It was found that P-glycoprotein is expressed in the PC-9-Br line which may explain the lack of efficacy in conventional chemotherapeutic agents. Vascularity is increased in regions of tumor, indicating that typical neoplastic angiogenesis occurs, which may be targeted by such agents as Notch- 4 inhibitors and bevacizumab. Finally, ${ }^{14} \mathrm{C}$-AIB permeability was increased to levels similar to that of breast-brain metastases, and lower values of passive permeability were associated with therapy that increased median survival. Tumor size and fold increases in markers were not correlated, much like in the breast-brain models. Lastly, smaller tumor sizes were associated with therapy that increased median survival.

With the efficacy of etirinotecan pegol in three cancer lines established, two other novel chemotherapeutic agents were evaluated in these models. Pegylated liposomal 
doxorubicin improved median survival over vehicle in the MDA-MB-231 model, but not the PC-9 model. Nanoparticle albumin-bound paclitaxel (nab-paclitaxel) did not improve survival in the MDA-MB-231 model.

\subsection{Future Studies}

Future studies, based on the results within this dissertation, include:

1. We have found that pegylated irinotecan (etirinotecan pegol) crosses the BTB and preferentially accumulates in tumors of breast-brain metastases, acting as a depot and providing sustained SN-38 levels. When compared to conventional chemotherapy and vehicle, etirinotecan pegol significantly prolonged survival and controlled tumor burden. A Phase 3 clinical study with etirinotecan pegol is recruiting patients to correlate these findings in breast cancer patients with brain metastases (NCT02915744). Unfortunately, etirinotecan pegol did not extend survival in the preclinical EGFR-mutated lung-brain metastatic line compared to conventional lung cancer therapy, or newly introduced targeted EGFR inhibitors. We also found that etirinotecan pegol has similar effects in a preclinical intracranial glioblastoma model compared to vehicle therapy. Future studies with etirinotecan pegol should evaluate its efficacy in the preclinical $E M L 4 / A L K$ fusion (NCI-H2228, NCI-H3122) and KRAS mutated (A549) lung cancer models, as well as its efficacy compared to conventional glioblastoma chemotherapy.

2. We observed that the EGFR-mutated PC-9-Br lung-breast metastatic model is similarly permeable to the passive diffusion marker ${ }^{14} \mathrm{C}$-AIB as some preclinical breast-brain metastatic models, despite being significantly smaller. 
Smaller tumor volumes and lower ${ }^{14} \mathrm{C}$-AIB permeability is associated with therapy that prolongs median survival. The presence of efflux pumps such as P-glycoprotein is prominent in the PC-9-Br line and inhibits chemotherapy from entering tumor regions. Future studies should evaluate ${ }^{14} \mathrm{C}$-AIB permeability and efflux pump effects on chemotherapy in the preclinical EML4/ALK fusion (NCI-H2228, NCI-H3122) and KRAS mutated (A549) lung cancer models, and evaluate if drug therapy changes tumor properties.

3. We observed that while albumin-bound paclitaxel (Abraxane) did not improve median survival in the preclinical 231-Br model, pegylated liposomal doxorubicin (Doxil) extended median survival by nearly 20\%. Conversely, Doxil had no effect on median survival in the PC-9-Br model. Future studies should evaluate Doxil in the HER2 ${ }^{+}$breast-brain metastatic model, EML4/ALK fusion (NCI-H2228, NCI-H3122) and KRAS mutated (A549) lung cancer models, and glioblastoma.

Current therapeutic options for treating brain metastases include surgery, whole-brain or stereotactic radiosurgery, and chemotherapy. Incremental advances have improved radiation therapy, but significant advances have been made in chemotherapy. The creation of targeted chemotherapy against oncogenic mutations, especially those that penetrate the blood-brain and blood-tumor barriers, have clinically demonstrated improvements in median survival in both in-vivo and patient settings. In combination with novel chemotherapeutic dosage forms, the outlook of brain metastases treatment is promising. 